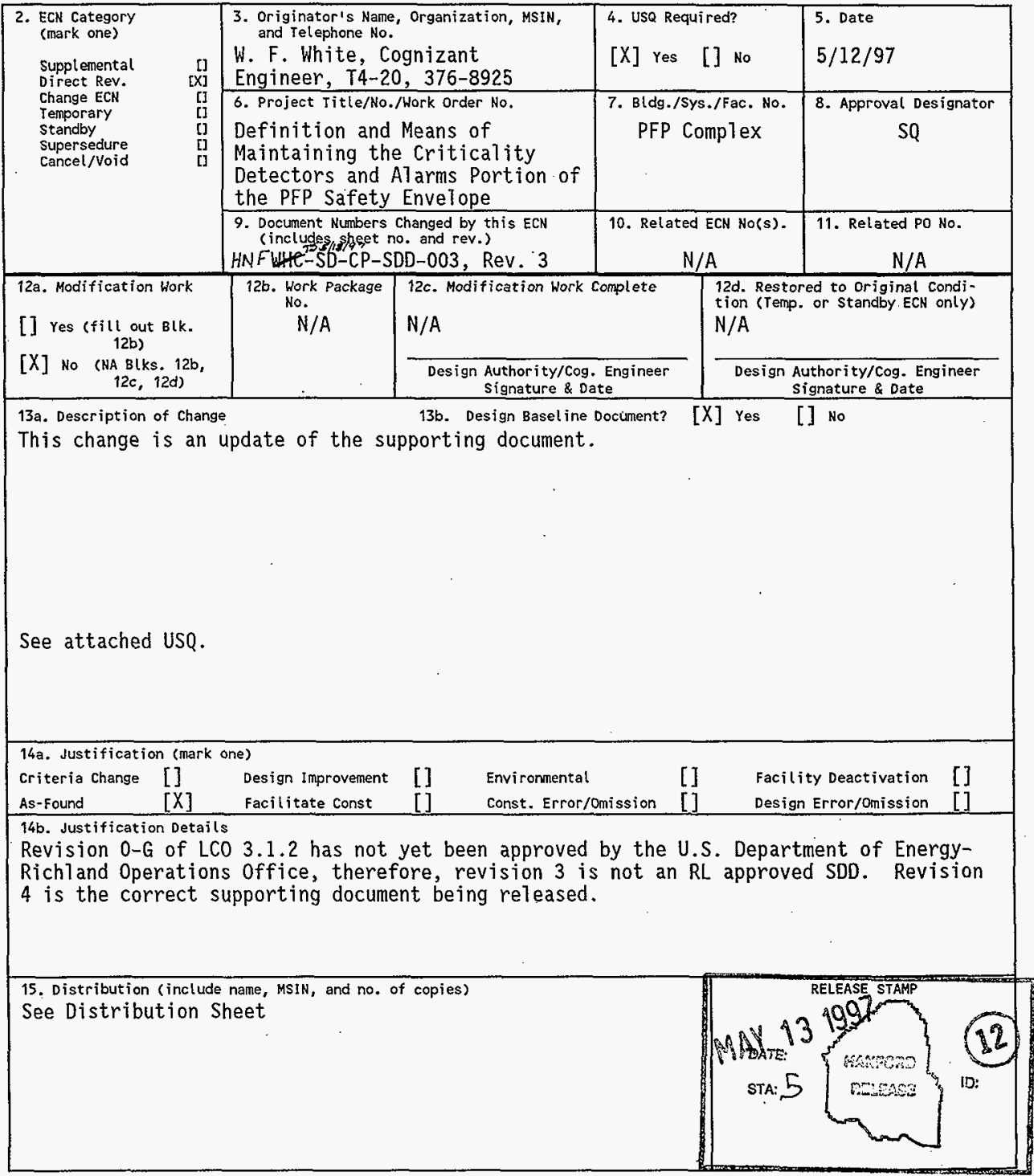


ENGINEERING CHANGE NOTICE

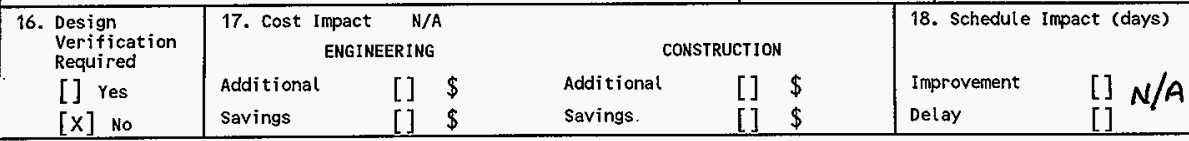

19. Change Impact Review: Indicate the related documents (other than the engineering documents identified on side 1 ) that will be affected by the change described in Block 13 . Enter the affected document number in Block 20.

SDD/DD
Funetional Design Criteria
Operating Specification
Criticality Specification
Conceptual Design Report
Equipment Spec.
Const. Spec.
Procurement Spec.
Vendor Information
OM Manual
FSAR/SAR
Safety Equipment List
Radiation Work Permit
Environmental Impact Statement
Environmental Report
Environmental Permit

$\begin{array}{lll}\text { [] } & \text { Seismic/Stress Analysis } & \text { Stress/Design Report } \\ {[]} & \text { Interface Control Drawing } & \\ {[]} & \text { Calibration Procedure } & \\ {[]} & \text { Installation Procedure } & \\ {[]} & \text { Maintenanee Procedure. } & \\ {[]} & \text { Engineering Procedure } \\ {[]} & \text { Operating Instruction . } \\ {[]} & \text { Operating Procedure } \\ {[]} & \text { Operational Safety Requirement } \\ {[]} & \text { IEFD Drawing } \\ {[]} & \text { Cell Arrangement Drawing } \\ {[]} & \text { Essential Material Specification } \\ {[]} & \text { Fac. Proc. Samp. Schedule } \\ {[]} & \text { Inspection Plan } \\ {[]} & \text { Inventory Adjustment Request }\end{array}$

[]
[]
[]
[]
[]
[]
[]
[]
[]
[]
[]
[]
[]
[]
[]
[]

Tank Calibration Manual

Health Physics Procedure

Spares Multiple Unit Listing

Test Procedures/Specification

Component Index

ASME Coded item

Human Factor Consideration

Computer Software

Electric Circuit Schedule

ICRS Procodure

Process Control Manual/Plan

Process Flow Chart

Purchase Requisition

Tickler File

N/A

20. Other Affected Documents: (NOTE: Documents listed below will not be revised by this ECN.) Signatures below indicate that the signing organization has been notified of other affected documents listed below. Document Number/Revision Document Number/Revision Document Number Revision N/A

21. Approvals

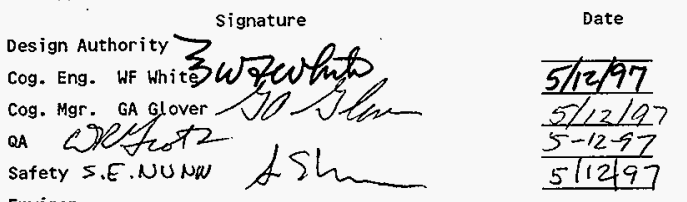

Environ.

Design Agent
$P E$
$Q A$
Safety
Designature
Environ.
Other

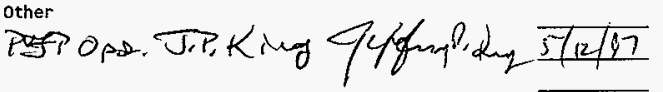

DEPARTMENT OF ENERGY

Signature or a Control Number that tracks the Approval signature

ADDITIONAL 


\section{UNREVIEWED SAFETY QUESTION (USQ) \\ SCREENING AND EVALUATION}

1. Identification Number: $\mathrm{ECN} 634415$

USO SCREENING

Page 1 of 2

2. ritle: WHC-SD-CP-SDD-003, "Definition and means of maintaining the Criticality Detectors and Alarms Portion of the PFP Safety Envelope"

\section{DESCRIPTION}

The changes to this supporting document clarify statements and update the current status of the system.

INSTRUCTIONS: Respond to each question and provide justification for each response. A restatement of the question does not constitute a satisfactory justification or basis. An adequate justification provides sufficient explanation such that an independent reviewer could reach the same conclusion based on the information provided [DOE 5480.21, 10.e.1].

QUESTIONS

1. Does the proposed change or occurrence represent a change to the facility or procedures as described in the Authorization Basis?
[] N/R
$[X]$ No
[] Yes/Maybe

BASIS: The changes described are administrative in nature, WHC-SD-CP-OSR-010, "PFP Operating Safety Requirements," Appendix A, "Bases," Section B 3.I "Criticality Safety System," describes the bases for the LCO'S and the surveillance requirements. The changes described do not affect these bases.

2. Does the proposed change or occurrence represent conditions that have not been analyzed in the Authorization Basis? [] N/A [X] No. [] Yes/Maybe

BASIS: There are no physical changes. The accident scenarios in chapter. 9 of the PFP FSAR (WHC-SD-CP-SAR-021) are not affected by the changes described.

3. Does the proposed change represent a test or experiment Nor described in the Authorization Basis that may affect the safe operation of the facility?

[X] N/A [] No [] Yes/Maybe

Basis: Test and experiments are not involved.

4. Does the proposed change or occurrence represent a change to the Technical Safety Requirements or a reduction in the margin of safety defined in the Technical Safety Requi rements?

[] N/A [X] No [] Yes/Maybe

BASIS: No changes which will affect the OSR (WHC-SD-CP-0SR-010), 1COS, or SRs are involved. 


\section{UNREVIEWED SAFETY QUESTION (USQ) \\ SCREENING AND EVALUATION}

\begin{tabular}{l|c|c|c|}
\hline 1. Identification Number: & ECN 634415 & USO SCREENING & Page 2 of 2 \\
\hline
\end{tabular}

2. Title: WHC-SD-CP-SDD-003, "Definition and means of maintaining the Criticality Detectors and Alarms Portion of the PFP Safety Envelope"

USQE \#1. L. E. Edvalson

(Print Name)
USQE \#2 G. A. G]over

(Print Name)

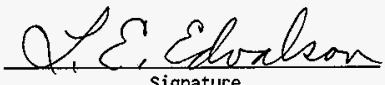

signature

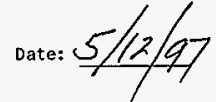

Date:

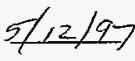

If there is a YES/MAYBE response to questions $1,2,3$, or 4, then a USQ Evaluation must be completed.

The following guidance should be considered when completing this sareening. This guidance should not be considered allinclusive; additional factors may need to be considered depending on the nature of the proposed change.

Does the proposed change:

1) Modify, add, or delete a safety class function of a structure, system or component stated in the authorization basis?

2) Alter the design of a structure, system or component as described in the authorization basis?

3) Modify, add, or delete the description of operation, operating environment, or analyses of any system or component described in the authorization basis?

4) Modify, add, delete or conflict with any of the design bases stated in the authorization basis?

5) Conflict with the principle or general design criteria stated in the authorization basis?

6) Modify, add, or delete any plant design features described in the authorization basis?

7) Modify, add, or delete a flow diagram or facility drawing provided in the authorization basis?

8) Create the potential for new system or component interactions (e.g., seismic, electrical breaker coordination)? 


\title{
DEFINITION AND MEANS OF MAINTAINING THE CRITICALITY DETECTORS AND ALARMS PORTION OF THE PFP SAFETY ENVELOPE
}

\author{
W. F. White
}

Babcock and Wilcox Hanford Co.. Richland, WA 99352

U.S. Department of Energy Contract DE-AC06-96RL13200

EDT/ECN: $\quad$ ECN -634415

Org Code: 15510

B\&R Code: EW7002000

UC: UC-506

Charge Code: $K 6058$

Total Pages: 95

Key Words: Criticality Alarm System, Authorization Basis, Safety Envelope, OSR, TSR

Abstract: The criticality Alarm system (CAS) provides continuous detection for high radiation (criticality) events, and automatically initiates an evacuation signal to affected personnel. The Safety Envelope (SE) for PFP includes the necessary equipnent and the required procedures to ensure the CAS is capable of performing its intended function.

This document provides the definition and means of maintaining the SE for PFP related to the CAS. This document also identifies and provides a justification for those portions of the CAS excluded from the PFP Safety Envelope.

\section{TRADEMARKS}

Allen-Bradley is a registered trademark of the Allen-Bradley Company, Inc., Milwaukee, WI

Hoffman is a registered trademark of First Bank National Association, Minneapolis, MN

North American is a registered trademark of North American Lighting, Inc., Flora, IL

Potter \& Brumfield is a registered trademark of Potter \& Brumfield, Inc.. Princeton, IN

Selectone is a registered trademark of Federal Sign and Signal Corp., Oak Brook, IL

Square D is a registered trademark of Square D Company, Palatine, IL

Vibratone is a registered trademark of Federal Sign and Signal Corp., Oak Brook, IL

TRADEMARK DISCLAIMER. Reference herein to any specific comercial product, process, or service by trade name, trademark, manufacturer, or otherwise, does not necessarily constitute or imply its endorsement, recommendation, or favoring by the United States Government or any agency thereof or its contractors or subcontractors.

Printed in the United States of America. To obtain copies of this document, contact: Document Control Services, P.O. Box 950, Mailstop H6-08, Richland WA 99352, Phone (509) 372-2420; Fax (509) 376-4989.

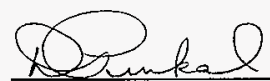

Release Approval

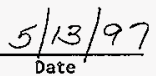

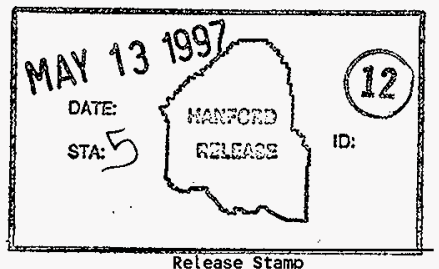

Release Stamp 


\section{RECORD OF REVISION}

(1) Document Number

HNF-SD-CP-SDD-003

Page 1

(2) ritle

Definition and Means of Maintaining the Criticality Detectors and Alarms Portion of the PFP Safety Envelope

CHANGE CONTROL RECORD

\begin{tabular}{|c|c|c|c|c|}
\hline \multirow{2}{*}{ (3) } & \multirow{2}{*}{ Revision } & \multirow{2}{*}{ (4) Description of Change - Replace, Add, and Delete Pages } & \multicolumn{2}{|c|}{ Authorized for Release } \\
\hline & & & (5) Cog. Engr. & (6) $\mathrm{Cog} . \mathrm{Mgr}$. \\
\hline & & (7) & & \\
\hline & 0 & $\begin{array}{l}\text { WHC-SD-CP-SDD-003, Rev. O EDT 140635, } \\
2 / 1 / 93\end{array}$ & & \\
\hline & 1 & $\begin{array}{l}\text { Direct revision. Revision } 1 \text { replaces } \\
\text { revision } 0 \text { in its entirety. Revision } 1 \mathrm{ECN} \\
\text { is } 605551 \text {. }\end{array}$ & CR Allen & GA Glover \\
\hline & 2 & $\begin{array}{l}\text { Direct revision. Revision } 2 \text { replaces } \\
\text { revision } 1 \text { in its entirety. Revision } 2 \text { ECN } \\
\text { is } 706855 \text {. Changes reflect FSAR changes } \\
\text { and current configuration of system. }\end{array}$ & CR Allen & GA Glover \\
\hline & 3 & $\begin{array}{l}\text { Direct revision. Supporting required } \\
\text { annual updated ECN } 634395 \text {. }\end{array}$ & WF White & GA Glover \\
\hline & 4 & $\begin{array}{l}\text { Direct revision. Supporting document } \\
\text { revision } 3 \text { was inadvertently rejeased } \\
\text { Correct document is release } 4 A .557974 \text {. }\end{array}$ & $\begin{array}{l}\text { WF White } \\
\text { wPowhths/4/87 }\end{array}$ & GA G1over $5 / 12 / 97$ \\
\hline & & per ECN - 634415 & & \\
\hline & & & & \\
\hline & & & & \\
\hline & & & & \\
\hline & & & & \\
\hline & & & & \\
\hline & & & & \\
\hline & & & & \\
\hline & & & & \\
\hline & & & & \\
\hline & & & & \\
\hline & & & & \\
\hline & & & & \\
\hline & & & & \\
\hline & & & & \\
\hline & & & & \\
\hline & & & & \\
\hline & & & & \\
\hline & & & & \\
\hline & & & & \\
\hline & & & & \\
\hline & & & & \\
\hline
\end{tabular}


Table of Contents

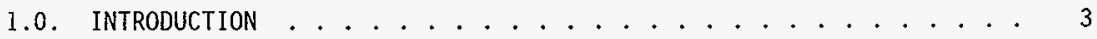

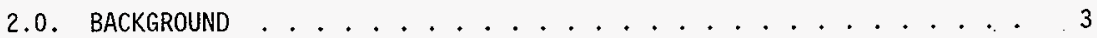

3.0. SYSTEM REQUIREMENTS ................. . . . 4

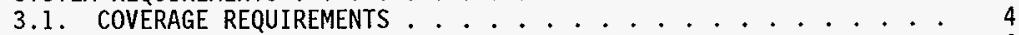

3.2. FUNCTIONAL REQUIREMENTS . . . . . . . . . . . . . . . 4

3.3. OPERABILITY REQUIREMENTS .............. . . 5

3.4. OPERATIONAL SAFETY REQUIREMENTS ............ 5

3.5. DESIGN REQUIREMENTS ............... 5

4.0. SAFETY ENVELOPE EQUIPMENT . . . . . . . . . . . 5

5.0. SAFETY ENVELOPE PROCEDURES . . . . . . . . . . . . . 7

5.1. MAINTENANCE PROCEDURES ............... . . . . 7

BB2. ALARM RESPONSE PROCEDURES . . . . . . . . 8

5.3. OPERATING PROCEDURES ............... . . . 8

6.0. SAFETY ENVELOPE SURVEILlANCE REQUIREMENTS . . . . . . . . . . 8

REFERENCES ............................. 11

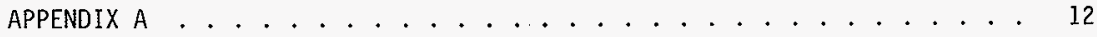

APPENDIX $8 \ldots \ldots \ldots \ldots$

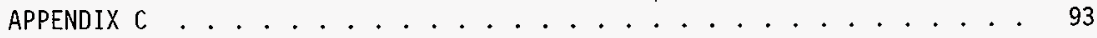

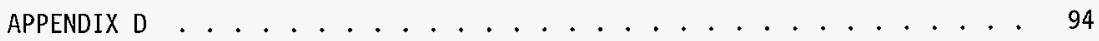




\subsection{INTRODUCTION}

The purpose of this document is to provide the definition and means of maintaining the Safety Envelope (SE) related to the Criticality Alarm System (CAS). This document provides amplification of the Limiting Condition for Operation (LCO) described in the Plutonium Finishing Plant (PFP) Operational Safety Requirements (OSR), WHC-SD-CP-OSR-010, Rev. 0, 1994, Section 3.1.2, "Criticality Detectors and Alarms."

This document, with its appendices, provides the following:

1. System functional requirements for determining system operability (Section 3 ).

2. A list of annotated system block diagrams which indicate the safety envelope boundaries (Appendix C).

3. A 1ist of the Safety Class 1 and 2 Safety Envelope (SC-1/2 $S E)$ equipment for input into the Master Component Index (Appendix B).

4. Functional requirements for individual SC-1/2 SE components, including appropriate setpoints and process parameters (Section 6 and Appendix A).

5. A list of the operational, maintenance and surveillance procedures necessary to operate and maintain the SC-1/2 SE components as required by the LCO (Section 6 and Appendix A).

\subsection{BACKGROUND}

The CAS provides continuous detection for high radiation (criticality) events and automatically initiates an evacuation signal to affected personnel. The CAS also establishes the approximate time of the criticality event.

There are two methods used to detect a criticality event at PFP. A gamma detection system is the predominant method and is used throughout the facility. In addition, a neutron detection system is used in the 2736-Z Building. Criticality detection is divided into 9 zones within the PFP complex. Each zone is serviced by several detectors, a criticality alarm panel, backup power supply, and annunciators.

Eight of these zones ( 1 through 7 and 9 ) are intertied such that the detection of a criticality event by one criticality alarm panel initiates evacuation signals in all eight zones. Zone 8 services the 241-Z Building, which is physically separate from the rest of the 
facility, and is not intertied to the other zones. Additional descriptions of the zones, criticality alarm components, and associated testing and calibration requirements are contained in WHC-IP-1115, Nuclear Criticality Alarm Manual for PFP.

\subsection{SYSTEM REQUIREMENTS}

\subsection{COVERAGE REQUIREMENTS}

WHC-CM-4-29, Section 11 requires a CAS to be insta1led and maintained operational for all fissionable material facilities and for fissionable material outside the core of nuclear reactor facilities, unless one of the following criteria is met:

1. A CAS is not required when a documented safety analysis shows that it is not needed due to the physical form and isotopic distribution of fissionable material. Such analyses shatl be approved by the independent safety review organization.

2. A CAS is not required when intervening shielding ensures that the maximum calculated dose in occupied locations will not exceed 25 rems whole body exposure or equivalent from the maximum accident of concern. Detection without facility evacuation is required in shielded areas in accordance with WHC-CM-4-29, Nuclear Criticality Safety Manual, Section 11.0, "Criticality ATarm System," Subsection 5.2.2, "Shielded Areas."

3. A CAS is not required when fissionable material packaging/shipping requirements have been complied with, and the material is either awaiting transport or in route in sealed, approved shipping containers.

PFP Operational Safety Requirement, LCO 3.1.2, "Criticality Detectors and Alarms" implements these requirements for PFP.

\subsection{FUNCTIONAL REQUIREMENTS}

WHC-CM-5-8, PFP Administration, Section 3.2, "Criticality Alarm System;" and WHC-CM-4-29, Section 11 require that the design, location and setpoint adjustment of the CAS shall provide detection of a criticality event and automatically signal evacuation immediately. Additionally, WHC-CM-4-29, Section 11, paragraph 5.3 , requires that each system shall include monitoring circuits to detect a malfunction or loss of primary power. Malfunction or power loss shall be indicated by visual and/or 
audible signals. The status of the system shall be constantly indicated in a designated, occupied location.

\subsection{OPERABILITY REQUIREMENTS}

Minimum operability of the CAS is defined as two functional detector/module pairs within one detector station (normally consisting of three detector/module pairs) provided all other SE components are functional. WHC-CM-5-8, Section 3.2, Subsection 4.4, "Operability" further defines the operability requirements for the CAS.

\subsection{OPERATIONAL SAFETY REQUIREMENTS}

The Operational Safety Requirements associated with the criticality alarm system are contained in WHC-SD-CP-OSR-010, Section 3.1.2. The requirement for this $L C O$ is:

LCO 3.1.2 The Criticality ATarm System (CAS) shaTl be OPERABLE.

\subsection{DESIGN REQUIREMENTS}

The design requirements for the criticality alarm system are contained in WHC-CM-4-29, Section 11, Subsection 5.3, "Design Requirements". This document will not address comp 1 iance to all the design requirements. Only the components necessary to fulfill OSR requirements are addressed. The CAS is designed such that 2-out-of $N$ (where $N \geq 3$ ) detectors must be tripped to initiate a criticality evacuation signal. The system contains audio and visual alarm annunciators necessary to inform affected personnel of the need to evacuate. The system also includes monitoring circuits to detect malfunction or loss of power and will automatically switch to backup power in the event of loss of normal power.

\subsection{SAFETY ENVELOPE EQUIPMENT}

Safety Class 1 and 2 Safety Envelope (SC-1/2 SE) equipment are those components necessary to ensure the CAS is capable of performing its minimum functional requirements as specified in Section 3.0 of this document. This is in support of LCO 3.1.2.

The block diagram defining the CAS SC-1/2 SE boundary is shown on drawing H-2-28883, Sheet 1, "Criticality Alarm System Block Diagram and Drawing Index." Appendix B of this document lists the CAS SC-1/2 SE equipment by individual Zones. 
SC-1/2 SE equipment included within a typical CAS Zone are the following:

1. Criticality Detector Station(s) consisting of a minimum of three criticality alarm detectors (CADs) (either gamma or neutron).

2. Criticality Alarm Panel (CAP) containing the following: One Criticality Alarm Detection Module (CADM) per CAD One Criticality Alarm Logic Module (CALM) serving up to six CADMs

One Criticality Alarm Relay Module (CARM)

One Audible Signal Generator (ASG)

Intertie Relay(s)

3. Evacuation Annunciators:

Criticality Alarm Horn(s) (CAH)

Criticality Alarm Flasher(s) (CAF), where required

4. Backup Power:

Battery and Battery Charger

Uninterruptible Power Supply (UPS) for CAP 7 only.

5. Transportable Criticality Alarm Systems when placed in service to satisfy requirements of LCO 3.1.2.

The CAS remote monitoring system (multiplexer (MUX) units, Criticality Alarm Annunciators (CAA), and AC power loss relays) is part of the PFP Safety Class 3 Safety Envelope (SC-3 SE). These SSCS are not essential to preventing or alarming a nuclear criticality. They provide remote indication of Criticality Alarm System operability and work with other methods to satisfy LCO 3.1 .2 and ensure continued operability of the CAS. The other methods of ensuring operability include the redundancy designed into the CAS (and included in the SC-1/2 SE); the monthly, quarterly, and annual surveillances required by LCO 3.1.2; and the once per shift CAP surveillance in procedure $20-100-400$.

A11 of the SC-3 SE SSCs, except the loss of power relays, are functionally tested by Safety Envelope Surveillance (ZSE) procedures. An annual functional test of the loss of power relays will be developed and performed under the B631 Phase 2 Project. Also, administrative procedures require hourly CAP surveillances when the CAS remote monitoring system is not functiona?. These procedures heip to ensure the CAS remains operable at all times. 


\subsection{SAFETY ENVELOPE PROCEDURES}

Procedures provide administrative control over the maintenance and calibration of the Criticality Alarm System to ensure operability of safety envelope components. Procedures also provide proper responses to alerts or alarms resulting from component failure, loss of power, or the detection of a nuclear criticality.

\subsection{MAINTENANCE PROCEDURES}

The procedures which satisfy the surveillance requirements for maintenance calibration and functional testing of CAS safety envelope equipment are identified in Section 6.0 of this document.

Non-LCO maintenance procedures related to the CAS are:

1-ZM-008, "Functional Testing of Transportable Criticality Alarm Systems"

1-ZM-023, "Restoring Criticality Alarm System to Normal Operation After AC Power Loss- System 99A"

1-ZM-030, "Neutron Criticality Alarm System Restoration After Power Loss-System 99A"

ZSR-99A-002, "Criticality Alarm System Sound Level Test" (Refer to WHCCM-5-8 Volume 1 section 3.2 paragraph 5.4, "Alarm Horn Sound Level Test").

This procedure provides the annual test of all the CAS horns including sound level measurements (in decibels) at specific locations within the facility. If needed, this test is performed at mid-interval (see WHC-CM-5-8 Volume 1 section 3.2 paragraph 5.4.1). 


\subsection{ALARM RESPONSE PROCEDURES}

Response procedures for CAS alarm signals are contained in WHC-CM-5-8, Section 3.2. Actions are provided for each of the following alarm conditions:

1. CALM/CARM Alarm or Evacuation (AH-00-GAH) Signal.

2. CALM/CARM Alert.

3. Detector Failure (Yellow Indicating Light).

4. Detector Power Failure (White Indicating Light).

5. Combination of Detector/Power Failure(s).

\subsection{OPERATING PROCEDURES}

Operating procedure 20-100-400, "Perform Nuclear Operations Surveillance and Responses," includes a local surveillance once each shift of each CAP to verify CAS operability.

\subsection{SAFETY ENVELOPE SURVEILLANCE REQUIREMENTS}

The surveillance requirements in $L C O 3.1 .2$ for the Criticality $A 1$ arm System are:

\section{SURVEILLANCE}

FREQUENCY

SR 3.1.2.1 VERIFY OPERABILITY of each detector and detector module response to a radiation check source.

SR 3.1.2.2 VERIFY OPERABILITY of the CAS battery and uninterruptible power supply systems.

Monthiy

Monthly

SR 3.1.2.3 VERIFY actuation of Audible Sound Generator audible and visual alarms.

Quarteriy

SR 3.1.2.4 VERIFY OPERABILITY of the CAS Logic and Relay Modules.

Quarteriy

SR 3.1.2.5 CALIBRATE each CAS detector and detector module. Annually 
ZSE-99A-001, "Criticality Alarm System (CAS) Check out and Audible/Visual Alarm Test"

This procedure is performed quarterly to verify the CAS gamma detector visuai and audible alarm systems operate as designed. The procedure verifies that each Criticality Alarm Detector Module (CADM), Criticality Alarm Logic Module (CALM), Criticality Alarm Relay Module (CARM), Audible Signal Generator (ASG) and alarm horn operate properly.

Procedure ZSE-99A-001 is performed to satisfy Surveillance Requirement 3.1.2.3.

ZSE-99A-002, "Criticality Alarm System, Neutron Criticality Detector System and Alarm Functional Test"

This procedure is performed monthly to verify the CAS neutron detectors respond properly to a neutron check source. The procedure is also performed quarterly to verify the CAS neutron detector visual and audible alarm systems operate as designed.

Procedure ZSE-99A-002 is performed to satisfy Surveillance Requirements 3.1.2.1 and 3.1.2.3.

ZSE-99A-006, "Monthly Inspection of Criticality Alarm System Batteries"

This procedure is performed monthly to verify CAS Battery Systems are capable of performing as designed by measuring the electrolyte level, specific gravity and cell voltage for each cell.

Procedure ZSE-99A-006 is performed to satisfy Surveillance Requirement 3.1.2.2.

ZSE-99A-007, "Topaz UPS n Monitor - 2736-ZA In-Service-Test"

This procedure is performed monthly to verify the TOPAZ uninterruptible power supply (UPS) in building 2736-ZA operates as designed. This UPS provides an uninterruptible 115 VAC to the neutron monitors for the vaults in 2736-Z.

Procedure ZSE-99A-007 is performed to satisfy Surveillance Requirement 3.1.2.2.

Note: Both ZSE-99A-006 and ZSE-99A-007 must be completed to satisfy Surveillance Requirement 3.1.2.2. 
ZSE-99A-011, "Criticality Alarm System Functional Test"

This procedure is performed monthly to verify each CAS gamma detector responds properly to a gamma check source. This. procedure al so verifies the proper operation of the CAS Logic and Relay Modules.

Procedure ZSE-99A-011 is performed to satisfy Surveillance Requirements 3.1.2.1 and 3.1.2.4.

ZSE-99A-021, "Criticality Alarm System Quarterly Calibration"

This procedure is performed quarterly to calibrate the CAS gamma detectors. The procedure uses a gamma calibration source to verify the proper calibration of each detector, and verifies the proper artificial background setting and the high radiation alarm setpoint.

Procedure ZSE-99A-021 is performed to satisfy Surve 711 ance Requirement 3.1.2.5. 


\section{REFERENCES}

ANSI/ANS 8.3. American National Standard, "Criticality Accident Alarm System," 1986

WHC-CM-4-29 Nuclear Criticality Safety Manual

WHC-CM-5-8 PFP Administration Manual, Section 3.2, "Criticality Alarm System"

WHC-IP-1115 Nuclear Criticality Alarm Manual PFP

WHC-SD-CP-SAR-021 Plutonium Finishing Plant Final Safety Analysis Report, Rev. 0,1995

WHC-SD-CP-0SR-010 Plutonium Finishing Plant Operational Safety Requirements, Rev. O, 1994 


\section{APPENDIX A}

\begin{tabular}{|c|c|c|c|}
\hline \multicolumn{4}{|c|}{ OOSR ECO COMP UTANCE SHEET } \\
\hline \multicolumn{4}{|c|}{ 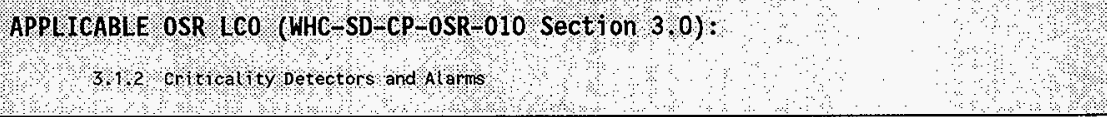 } \\
\hline \multicolumn{4}{|c|}{ LCO 3.1.2 The criticality Alarm System (CAS) shall be OPERABLE. } \\
\hline \multicolumn{4}{|l|}{$\begin{array}{l}\text { APPLICABILITY: All MOOES } \\
\text { ACTIONS: }\end{array}$} \\
\hline CONDITION & \multicolumn{2}{|c|}{ REQUIRED ACTION } & $\begin{array}{l}\text { COMPLETION } \\
\text { TIME }\end{array}$ \\
\hline $\begin{array}{l}\text { A. Single detector or detector module } \\
\text { inoperable for any detector station. }\end{array}$ & $\begin{array}{l}\text { A.1 } \\
\text { AND } \\
\text { A.2 }\end{array}$ & $\begin{array}{l}\text { Enter MODE 2, Limited } \\
\text { Plutonium Handling } \\
\text { Operations in the affected } \\
\text { area. Stop fissile } \\
\text { material (greater than } 15 \\
\text { grams) movements in the } \\
\text { affected area. } \\
\text { Repair or replace failed } \\
\text { detector or detector } \\
\text { module. }\end{array}$ & $\begin{array}{l}4 \text { Hours } \\
48 \text { Hours }\end{array}$ \\
\hline $\begin{array}{l}\text { A. A Criticality Alarm Panel (CAP) is } \\
\text { inoperable. }\end{array}$ & $\begin{array}{l}\text { B.1 } \\
\text { OR } \\
B .2\end{array}$ & 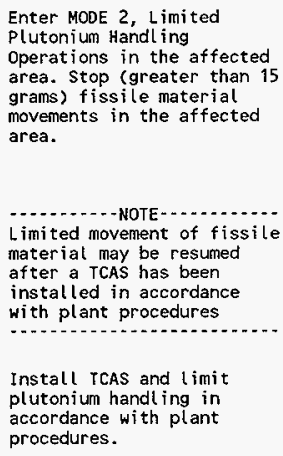 & Inmedi ately \\
\hline
\end{tabular}




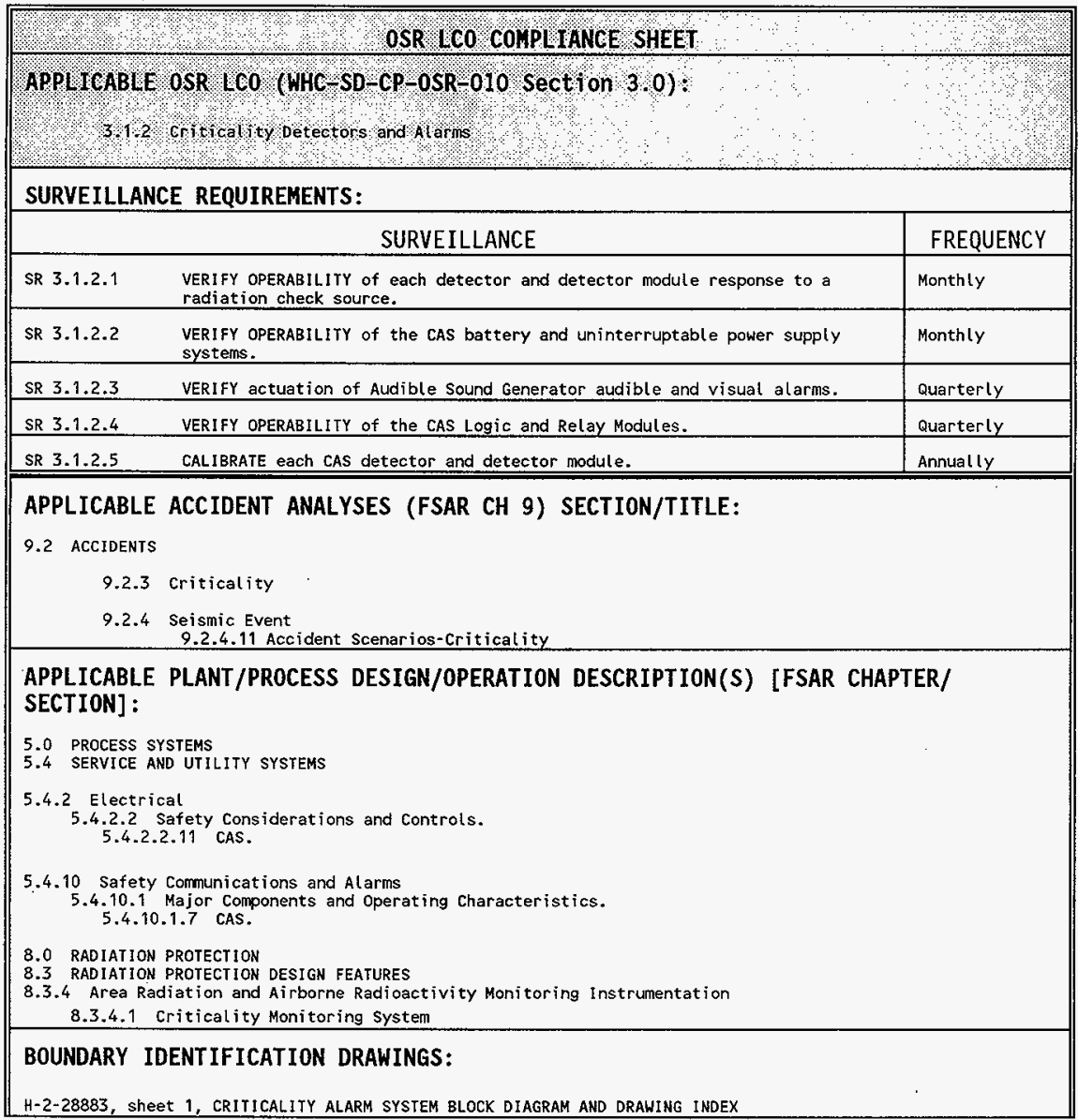




\begin{tabular}{|c|}
\hline V. \\
\hline 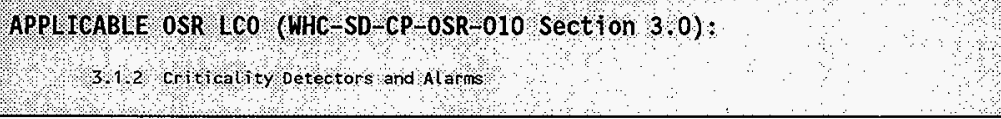 \\
\hline ESSENTIAL SYSTEM DRAWINGS: \\
\hline 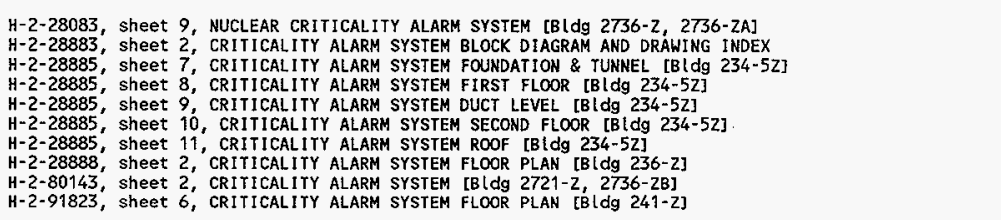 \\
\hline IMPEEMENT ING PROCEDURES/CONPL IANCE VERIFICATION \\
\hline OPERATING: $\quad 20-100-400$, "Perform Nuclear operations surveill ance and Responses" \\
\hline LABORATORY: NONE \\
\hline HEALTH PHYSICS: NONE \\
\hline OPERATING SPECIFICATION(S): NONE \\
\hline $\begin{array}{l}\text { ADMINISTRAT IVE: } \\
\text { WHC-CM-4-29, } \\
\text { WHC-CM-5-8, } \frac{\text { Nuclear Critical ity Safety Manual }}{\text { Fedministration Manual, Section } 3.2 \text {, CRITICALITY ALARM SYSTEM }} \\
\text { WHC-1P-1115, } \\
\text { WHCC-5-8 Vol } 1 \text {, PFP Administration Manual, Section } 3.2 \text { par. } 5.4 \text {, "Alarm Horn Sound Level Test" }\end{array}$ \\
\hline $\begin{array}{l}\text { MAINTENANCE: } \\
\text { NON-LCO } \\
\text { 1-ZM-023, "Restoring Criticality Alarm System to Normal Operation After AC Power Loss- System 99A" } \\
\text { 1-ZM-030, "Neutron Criticality System Restoration to Battery Back-up After Power Loss-System 99A" } \\
\text { 1-ZM-008, "Functional Testing of Transportable Criticality Alarm Systems" }\end{array}$ \\
\hline
\end{tabular}




\begin{tabular}{|c|c|c|c|}
\hline \multicolumn{4}{|c|}{ W } \\
\hline \multicolumn{4}{|c|}{ 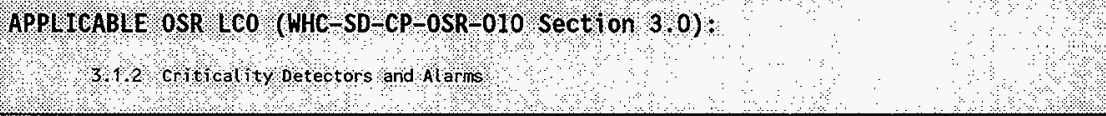 } \\
\hline $\begin{array}{l}\text { SURYEILLANCE } \\
\text { REQUIREMENTS }\end{array}$ & PROCEDURE NUMBER & DESCRIPTION & $\begin{array}{l}\text { PROCEDURE } \\
\text { FREQUENCY }\end{array}$ \\
\hline \multirow[t]{2}{*}{ SR 3.1.2.1 } & $\begin{array}{l}\text { ZSE-99A-011, "Criticality Alarm } \\
\text { System Functional Test" }\end{array}$ & $\begin{array}{l}\text { Verifies gamma detectors respond } \\
\text { properly to a check source. }\end{array}$ & Monthly \\
\hline & $\begin{array}{l}\text { ZSE }-99 \mathrm{~A}-002 \text {, "Neutron } \\
\text { Critjcality Detector System and } \\
\text { Alarm Functional Test" }\end{array}$ & $\begin{array}{l}\text { Verifies neutron detectors respond } \\
\text { properly to a check source. }\end{array}$ & Monthly \\
\hline \multirow[t]{2}{*}{ SR 3.1 .2 .2} & $\begin{array}{l}\text { ZSE-99A-006, "Monthly Inspection } \\
\text { of Criticality Alarm System } \\
\text { Batteries" }\end{array}$ & Verifies battery operability. & Monthly \\
\hline & $\begin{array}{l}\text { ZSE-99A-007, "Topaz UPS } n \\
\text { Honitor }-2736 \text { ZA } \\
\text { In-Service-Test" }\end{array}$ & Verifies UPS operability. & Monthly \\
\hline \multirow[t]{2}{*}{ SR 3.1.2.3 } & $\begin{array}{l}\text { 2SE-99A-001, "Criticality Alarm } \\
\text { System (CAS) Check out and } \\
\text { Audible/Visual Alarm Test" }\end{array}$ & $\begin{array}{l}\text { Verifies CAPs and alarm horn } \\
\text { operability. }\end{array}$ & Quarterly \\
\hline & $\begin{array}{l}\text { 2SE-99A-002, "Neutron } \\
\text { Criticality Detector System and } \\
\text { A larm Functional Test" }\end{array}$ & $\begin{array}{l}\text { Verifies neutron detector visual } \\
\text { and audible alarm operability. }\end{array}$ & Quarterly \\
\hline SR 3.1 .2 .4 & $\begin{array}{l}\text { ZSE-99A-011, "Criticality Alarm } \\
\text { system Functional Test" }\end{array}$ & $\begin{array}{l}\text { Verifies proper operation of CAS } \\
\text { Logic and Relay Modules. }\end{array}$ & Quarterly \\
\hline SR 3.1.2.5 & $\begin{array}{l}\text { 2SE-99A-021, "Criticality Alarm } \\
\text { System Quarterly Calibration" }\end{array}$ & Calibrates CAS ganma detectors. & Quarterly \\
\hline \multicolumn{4}{|c|}{ other s, None } \\
\hline \multicolumn{4}{|c|}{ NOTES/COMMENTS: None } \\
\hline
\end{tabular}




\author{
APPENDIX B \\ MASTER COMPONENT INDEX LISTING
}

\title{
APRLICABLE OSR ICO (MHC-SD-CP-0SR-010 Section 3.0):
}

3.12 crutical 1ty Detectors and Alarms

\section{OSR SYSTEH EQUIPHENT/COMPONENT IIST}

ZONE 1

\begin{tabular}{|c|c|c|c|}
\hline $\begin{array}{l}\text { MASIER } \\
\text { CONPONENT } \\
\text { HNDEX } \\
\text { NUNBER }\end{array}$ & 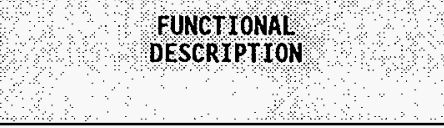 & $\begin{array}{l}\text { WAUFAOTURER } \\
\text { PART/HODEL } \\
\text { WUWBER } \\
\text { (DWg } H-2-28884 \text { ) }\end{array}$ & $\begin{array}{l}\text { ESSENISA } \\
\text { ORAWTHG } \\
\text { NUMBER }\end{array}$ \\
\hline ASG $-Z 1$ & $\begin{array}{l}\text { Audible Signal Generator. (Analog Type) } \\
\text { Generates an "AH-OO-GAH" electrical signal } \\
\text { that is wired to the critical ity } \\
\text { annunciator horns. Located in CAP-21. }\end{array}$ & Fabricated per H-2-72419 & $\begin{array}{l}H-2-28883 \text { sht } 1 \\
H-2-28883 \text { sht } 2 \\
H-2-28885 \text { sht } 8\end{array}$ \\
\hline$C A D-Z 1-1 A$ & $\begin{array}{l}\text { Criticality Alarm Detector, (Gamma). } \\
\text { Detects radiation resulting from potential } \\
\text { criticality excursions. Located in } 234-52 \text {, } \\
\text { Floor } 1 \text {, Room } 221 \text {. }\end{array}$ & $\begin{array}{l}\text { Nuclear Measurement Corp. } \\
\text { Model GA-2 TMO }\end{array}$ & $\begin{array}{l}H-2-28883 \text { sht } 1 \\
H-2-28883 \text { sht } 2 \\
H-2-28885 \text { sht } 8\end{array}$ \\
\hline$C A D-Z 1-18$ & $\begin{array}{l}\text { Criticality Alarm Detector. (Gamma). } \\
\text { Detects radiation resulting from potential } \\
\text { criticality excursions. Located in } 234-52 \text {, } \\
\text { Floor } 1 \text {, Room } 221 \mathrm{~A} \text {. }\end{array}$ & $\begin{array}{l}\text { Nuclear Measurement Corp. } \\
\text { Model GA-2 TMO }\end{array}$ & $\begin{array}{l}H-2-28883 \text { sht } 1 \\
H-2-28883 \text { sht } 2 \\
H-2-28885 \text { sht } 8\end{array}$ \\
\hline$C A D-Z 1-1 C$ & $\begin{array}{l}\text { Criticality Alarm Detector. (Gamma). } \\
\text { Detects radiation resulting from potential } \\
\text { criticality excursions. Located in } 234-52 \text {, } \\
\text { Floor 1, Room } 221 \mathrm{~A} \text {. }\end{array}$ & $\begin{array}{l}\text { Nuclear Measurement Corp. } \\
\text { Hodel GA-2 TMO }\end{array}$ & $\begin{array}{l}H-2-28883 \text { sht } 1 \\
H-2-28883 \text { sht } 2 \\
H-2-28885 \text { sht } 8\end{array}$ \\
\hline$C A D-21-2 A$ & $\begin{array}{l}\text { Criticality Alarm Detector. (Gamma). } \\
\text { Detects radiation resulting from potential } \\
\text { criticality excursions. Located in } 234-52 \text {, } \\
\text { Floor } 1 \text {, Corridor } 5 \text {. }\end{array}$ & $\begin{array}{l}\text { Nuclear Measurement Corp. } \\
\text { Model GA-2 TMO }\end{array}$ & $\begin{array}{l}H-2-28883 \text { sht } 1 \\
\text { H-2-28883 sht } 2 \\
\text { H-2-28885 sht } 8\end{array}$ \\
\hline$C A D-Z 1-2 B$ & $\begin{array}{l}\text { Criticality Alarm Detector. (Gama). } \\
\text { Detects radiation resulting from potential } \\
\text { critical ity excursions. Located in } 234-52 \text {, } \\
\text { floor } 1 \text {, Corridor } 5 \text {. }\end{array}$ & $\begin{array}{l}\text { Nuclear Measurement Corp. } \\
\text { Model GA-2 TMO }\end{array}$ & $\begin{array}{l}H-2-28883 \text { sht } 1 \\
H-2-28883 \text { sht } 2 \\
H=2-28885 \text { sht } 8\end{array}$ \\
\hline$C A D-Z 1-2 C$ & $\begin{array}{l}\text { Criticality Alarm Detector. (Gama). } \\
\text { Detects radiation resulting from potential } \\
\text { criticality excursions. Located in } 234-52 \text {, } \\
\text { Floor } 1 \text {, Corridor } 5 \text {. }\end{array}$ & $\begin{array}{l}\text { Nuclear Measurement Corp. } \\
\text { Model GA-2 TMO }\end{array}$ & $\begin{array}{l}H-2-28883 \text { sht } 1 \\
H-2-28883 \text { sht } 2 \\
H-2-28885 \text { sht } 8\end{array}$ \\
\hline$C A D-Z 1-3 A$ & $\begin{array}{l}\text { Criticality Alarm Detector. (Gamma). } \\
\text { Detects radiation resulting from potential } \\
\text { critical ity excursions. Located in } 234-52 \text {, } \\
\text { floor } 1 \text {, Room } 262 \text {. }\end{array}$ & $\begin{array}{l}\text { Nuclear Measurement Corp. } \\
\text { Model GA-2 TMO }\end{array}$ & $\begin{array}{l}H-2-28883 \text { sht } 1 \\
H-2-28883 \text { sht } 2 \\
H-2-28885 \text { sht } 9\end{array}$ \\
\hline$C A D-Z 1-3 B$ & $\begin{array}{l}\text { Criticality Alarm Detector. (Gamma). } \\
\text { Detects radiation resulting from potential } \\
\text { critical ity excursions. Located in } 234-52 \text {, } \\
\text { Floor } 1 \text {, Room } 262 \text {. }\end{array}$ & $\begin{array}{l}\text { Nuclear Measurement corp. } \\
\text { Model GA-2 TMO }\end{array}$ & $\begin{array}{l}H-2-28883 \text { sht } 1 \\
H-2-28883 \text { sht } 2 \\
H-2-28885 \text { sht } 9\end{array}$ \\
\hline$C A D-Z 1-3 C$ & $\begin{array}{l}\text { Critical ity Alarm Detector. (Gamma). } \\
\text { Detects radiation resulting from potential } \\
\text { criticality excursions. Located in } 234-52 \text {, } \\
\text { Floor } 1 \text {, Room } 262 \text {. }\end{array}$ & $\begin{array}{l}\text { Nuclear Measurement Corp. } \\
\text { Model GA-2 TMO }\end{array}$ & $\begin{array}{l}H-2-28883 \text { sht } 1 \\
H-2-28883 \text { sht } 2 \\
H-2-28885 \text { sht } 9\end{array}$ \\
\hline
\end{tabular}




\section{APPLICABLE 0SR 100 (HHC-SD-CP-0SR-010 sect on 3.0$)$ \\ 3.1 .2 cr ltical 1 ty Detectors and $A 1$ anms}

OSR SYSTEN EQUTPMENT/COHPONENT LIST

ZONE 1

\begin{tabular}{|c|c|c|c|}
\hline $\begin{array}{l}\text { MASTER } \\
\text { COMPONENT } \\
\text { NOMBEX }\end{array}$ & 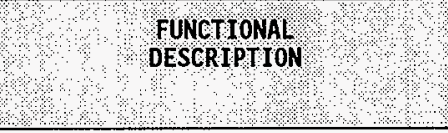 & $\begin{array}{l}\text { MANUFACTURER } \\
\text { PART/WODEL } \\
\text { NUNBER } \\
\text { (DWg } 1 \text { 2 } 28884)\end{array}$ & $\begin{array}{l}\text { ESSENTHA } \\
\text { DRALING } \\
\text { NGHBER }\end{array}$ \\
\hline$C A D-Z 1-4 A$ & $\begin{array}{l}\text { Criticality Alarm Detector. (Gamma). } \\
\text { Detects radiation resulting from potential } \\
\text { critical ity excursions. Located in } 234-52 \text {, } \\
\text { Floor } 1 \text {, Corridor } 2428 \text {. }\end{array}$ & $\begin{array}{l}\text { Nuclear Measurement Corp. } \\
\text { Model GA-2 TMO }\end{array}$ & $\begin{array}{l}H-2-28883 \text { sht } 1 \\
H-2-28883 \text { sht } 2 \\
H-2-28885 \text { sht } 8\end{array}$ \\
\hline$C A D-Z 1-4 B$ & $\begin{array}{l}\text { Criticality Alarm Detector. (Gamma). } \\
\text { Detects radiation resulting from potential } \\
\text { criticality excursions. Located in } 234-52 \text {, } \\
\text { Floor } 1 \text {, corridor } 242 B \text {. }\end{array}$ & $\begin{array}{l}\text { Nucl ear Measurement Corp. } \\
\text { Model GA-2 TMO }\end{array}$ & $\begin{array}{l}H-2-28883 \text { sht } 1 \\
H-2-28883 \text { sht } 2 \\
H-2-28885 \text { sht } 8\end{array}$ \\
\hline$C A D-Z 1-4 C$ & $\begin{array}{l}\text { Criticality Alarm Detector. (Gamma). } \\
\text { Detects radiation resulting from potential } \\
\text { critical ity excursions. Located in } 234-52 \text {, } \\
\text { Floor } 1 \text {, Corridor } 2428 \text {. }\end{array}$ & $\begin{array}{l}\text { Nucl ear Measurement Corp. } \\
\text { Model GA-2 TMO }\end{array}$ & $\begin{array}{l}H-2-28883 \text { sht } 1 \\
H-2-28883 \text { sht } 2 \\
H-2-28885 \text { sht } 8\end{array}$ \\
\hline$C A D-Z 1-5 A$ & $\begin{array}{l}\text { Criticality Alarm Detector. (Gamma). } \\
\text { Detects radiation resulting from potential } \\
\text { critical ity excursions. Located in } 234-52 \text {, } \\
\text { Floor } t \text {, Corridor } 4 \mathrm{~B} \text {. }\end{array}$ & $\begin{array}{l}\text { Nucl ear Measurement Corp. } \\
\text { Model GA-2 TNO }\end{array}$ & $\begin{array}{l}H-2-28883 \text { sht } 1 \\
H-2-28883 \text { sht } 2 \\
H-2-28885 \text { sht } 8\end{array}$ \\
\hline$C A D-Z 1-5 B$ & $\begin{array}{l}\text { Criticality Alarm Detector. (Gamma). } \\
\text { Detects radiation resulting from potential } \\
\text { critical ity excursions. Located in } 234-52 \text {, } \\
\text { Floor } 1 \text {, Corridor } 4 \mathrm{~B} \text {. }\end{array}$ & $\begin{array}{l}\text { Nuclear Measurement Corp: } \\
\text { Model GA-2 TMO }\end{array}$ & $\begin{array}{ll}H-2-28883 & \text { sht } 1 \\
H-2-28883 & \text { sht } \\
H-2-28885 & \text { sht } 8\end{array}$ \\
\hline$C A D-Z 1-5 C$ & $\begin{array}{l}\text { Criticality Alarm Detector. (Gamma). } \\
\text { Detects radiation resulting from potential } \\
\text { criticality excursions: Located in } 234-52 \text {, } \\
\text { Floor } 1 \text {, Corridor 4B. }\end{array}$ & $\begin{array}{l}\text { Mucl ear Measurement Corp. } \\
\text { Model GA-2 TMO }\end{array}$ & $\begin{array}{l}H-2-28883 \text { sht } 1 \\
H-2-28883 \text { sht } 2 \\
H-2-28885 \text { sht } 8\end{array}$ \\
\hline$C A D-Z 1-6 A$ & $\begin{array}{l}\text { Criticality Alarm Detector. (Gamm). } \\
\text { Detects radiation resulting from potential } \\
\text { criticality excursions. Located in } 234-57 \text {, } \\
\text { Floor 1, Room 192A. }\end{array}$ & $\begin{array}{l}\text { Nuclear Measurement corp. } \\
\text { Model GA-2 TMO }\end{array}$ & $\begin{array}{l}H-2-28883 \text { sht } 1 \\
H-2-28883 \text { sht } 2 \\
H-2-28885 \text { sht } 8\end{array}$ \\
\hline$C A D-Z 1-6 B$ & $\begin{array}{l}\text { Criticality Alarm Detector. (Garma). } \\
\text { Detects radiation resulting from potential } \\
\text { criticality excursions. Located in } 234-52 \text {, } \\
\text { Floor 1, Room } 192 \mathrm{~A} \text {. }\end{array}$ & $\begin{array}{l}\text { Nuclear Measurement Corp. } \\
\text { Model GA-2 TMO }\end{array}$ & $\begin{array}{l}H-2-28883 \text { sht } 1 \\
H-2-28883 \text { sht } 2 \\
H-2-28885 \text { sht } 8\end{array}$ \\
\hline$C A D-21-6 C$ & $\begin{array}{l}\text { Criticality Alarm Detector. (Gamma). } \\
\text { Detects radiation resulting from potential } \\
\text { criticality excursions. Located in } 234-52 \text {, } \\
\text { Floor 1, Room } 192 \mathrm{~A} \text {. }\end{array}$ & $\begin{array}{l}\text { Nuclear Measurement Corp. } \\
\text { Model GA-2 TMO }\end{array}$ & $\begin{array}{l}H-2-28883 \text { sht } 1 \\
H-2-28883 \text { sht } 2 \\
H-2-28885 \text { sht } 8\end{array}$ \\
\hline CADM-Z1-1A & $\begin{array}{l}\text { Criticality Alarm Detection Module. One } \\
\text { (1) per CAD. Provides dose rate } \\
\text { indication, calibration adjustments for the } \\
\text { CAD-CADM unit, and alarm functions. } \\
\text { Located in CAP-Z1. }\end{array}$ & $\begin{array}{l}\text { Nuclear Measurement Corp. } \\
\text { Model GA-2 TMO }\end{array}$ & $\begin{array}{l}\text { H-2-28883 sht } 1 \\
\text { H-2-28883 sht } 2 \\
H-2-28885 \text { sht } 8\end{array}$ \\
\hline
\end{tabular}




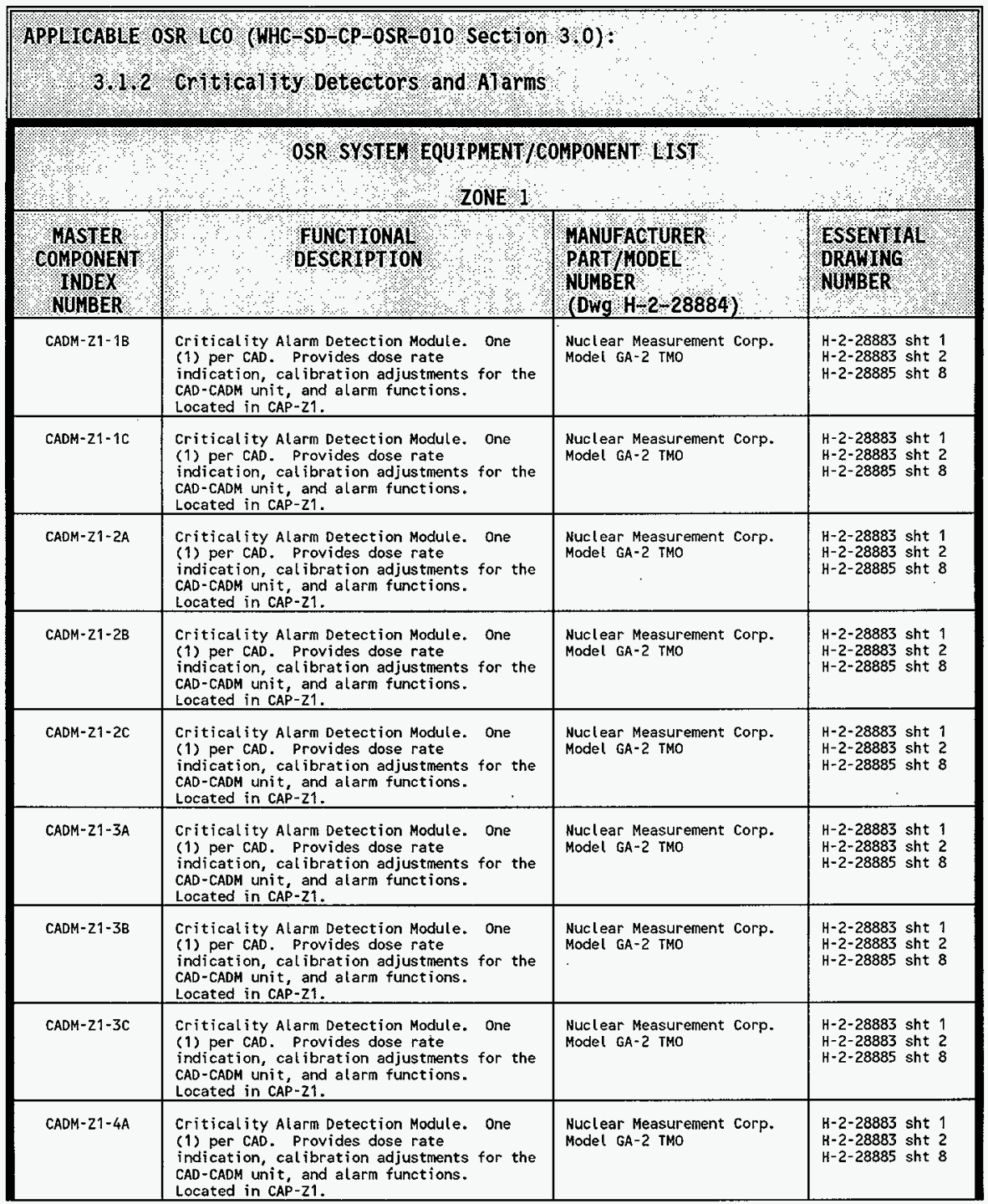




\begin{tabular}{|c|c|c|c|}
\hline \multicolumn{4}{|c|}{ 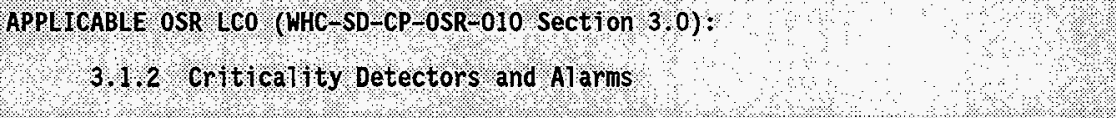 } \\
\hline $\begin{array}{ccc}2 \\
2\end{array}$ & 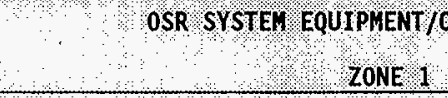 & $\begin{array}{l}\text { WONENT LTS } \\
\therefore \quad\end{array}$ & \\
\hline $\begin{array}{l}\text { WASTER } \\
\text { COMPONENT } \\
\text { WUNDEX }\end{array}$ & 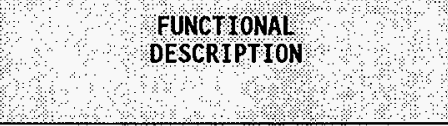 & $\begin{array}{l}\text { WAWUFACTURER } \\
\text { PART/HODEL } \\
\text { NUWBER } \\
\text { (DWOHH } 2-28884)\end{array}$ & $\begin{array}{l}\text { ESSEATHAL } \\
\text { DRAWIUG } \\
\text { NUMBER }\end{array}$ \\
\hline CADM-Z1-4C & $\begin{array}{l}\text { Criticality Alarm Detection Module. one } \\
\text { (1) per CAD. Provides dose rate } \\
\text { indication, calibration adjustments for the } \\
\text { CAD-CADM unit, and alarm functions. } \\
\text { Located in CAP-Z1. }\end{array}$ & $\begin{array}{l}\text { Nuclear Measurement Corp. } \\
\text { Model GA-2 TMO }\end{array}$ & $\begin{array}{l}H-2-28883 \text { sht } 1 \\
H-2-28883 \text { sht } 2 \\
H-2-28885 \text { sht } 8\end{array}$ \\
\hline CADM-Z1-5A & $\begin{array}{l}\text { Criticality ALarm Detection Modute. One } \\
\text { (1) per CAD. Provides dose rate } \\
\text { indication, catibration adjustments for the } \\
\text { CAD-CADM unit, and alarm functions. } \\
\text { Located in CAP-Z1. }\end{array}$ & $\begin{array}{l}\text { Nuclear Measurement Corp. } \\
\text { Model GA-2 TMO }\end{array}$ & $\begin{array}{l}H-2-28883 \text { sht } 1 \\
H-2-28883 \text { sht } 2 \\
H-2-28885 \text { sht } 8\end{array}$ \\
\hline CADM-Z1-5B & $\begin{array}{l}\text { Criticality Alarm Detection Module. One } \\
\text { (1) per CAD. Provides dose rate } \\
\text { indication, calibration adjustments for the } \\
\text { CAD-CADM unit, and alarm functions. } \\
\text { Located in CAP-21. }\end{array}$ & $\begin{array}{l}\text { Nuclear Measurement Corp. } \\
\text { Model GA-2 TMO }\end{array}$ & $\begin{array}{l}H-2-28883 \text { sht } 1 \\
H-2-28883 \text { sht } 2 \\
H-2-28885 \text { sht } 8\end{array}$ \\
\hline CADM-Z1-6A & $\begin{array}{l}\text { Criticality Alarm Detection Module. one } \\
\text { (1) per CAD. Provides dose rate } \\
\text { indication, calibration adjustments for the } \\
\text { CAD-CADM unit, and alarm functions. } \\
\text { Located in CAP-Z1. }\end{array}$ & $\begin{array}{l}\text { Nuclear Measurement Corp. } \\
\text { Model GA-2 TMO }\end{array}$ & $\begin{array}{l}H-2-28883 \text { sht } 1 \\
H-2-28883 \text { sht } 2 \\
H-2-28885 \text { sht } 8\end{array}$ \\
\hline CADM-21-6B & $\begin{array}{l}\text { Criticality Alarm Detection Module. One } \\
\text { (1) per CAD. Provides dose rate } \\
\text { indication, calibration adjustments for the } \\
\text { CAD-CADM unit, and alarm functions. } \\
\text { Located in CAP-Z1. }\end{array}$ & $\begin{array}{l}\text { Nuclear Measurement Corp. } \\
\text { Model GA-2 TMO }\end{array}$ & $\begin{array}{l}H-2-28883 \text { sht } 1 \\
H-2-28883 \text { sht } 2 \\
H-2-28885 \text { sht } 8\end{array}$ \\
\hline CADM-21-6C & $\begin{array}{l}\text { Criticality Alarm Detection Hodule. One } \\
\text { (1) per CAD. Provides dose rate } \\
\text { indication, calibration adjustments for the } \\
\text { CAD-CADM unit, and alarm functions. } \\
\text { Located in CAP-Z1. }\end{array}$ & $\begin{array}{l}\text { Nuclear Measurement Corp. } \\
\text { Model GA-2 TMO }\end{array}$ & $\begin{array}{l}H-2-28883 \text { sht } 1 \\
H-2-28883 \text { sht } 2 \\
H-2-28885 \text { sht } 8\end{array}$ \\
\hline $\mathrm{CAH}-\mathrm{Z1}-1$ & $\begin{array}{l}\text { Criticality Alarm Horn. Located in } \\
\text { Building } 234-52 \text { Corridor } 2 \text {. }\end{array}$ & $\begin{array}{l}\text { Audible signaling Device: } \\
\text { Federal signal Corp. Model } \\
300 \text { GC, } 24 \text { VAC/DC Selectone } \\
\text { Connector Board: FS Corp. } \\
\text { Model } 300 \text { CK }\end{array}$ & $\begin{array}{l}H-2-28883 \text { sht } 1 \\
H-2-28883 \text { sht } 2 \\
H-2-28885 \text { sht } 8\end{array}$ \\
\hline
\end{tabular}




\section{APPLTCABLE OSR LCO (WHC-SD-CP-0SR-010 Section 3.0 ) :}

\subsection{2 criticallty Detectors and Alarms}

\section{OSR SYSTEN EQUIPNENT/CONPONENT LIST}

ZONE 1

\begin{tabular}{|c|c|c|c|}
\hline $\begin{array}{l}\text { MASTER } \\
\text { COMPONENT } \\
\text { TNDEX } \\
\text { NUMBER }\end{array}$ & 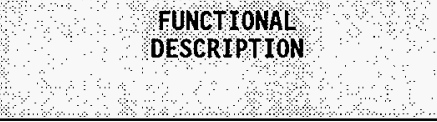 & $\begin{array}{l}\text { WANUFACTURER } \\
\text { PARTYWODEE } \\
\text { NUNBER } \\
\text { (DWG } \mathrm{H}-2-28884 \text { ) }\end{array}$ & $\begin{array}{l}\text { ESSENTIA } \\
\text { DRAW WG }\end{array}$ \\
\hline $\mathrm{CAH}-\mathrm{Z1-2}$ & $\begin{array}{l}\text { Criticality Alarm Horn. Located in } \\
\text { Building } 234-52 \text { Room } 124 \text {. }\end{array}$ & $\begin{array}{l}\text { Audible Signaling Device: } \\
\text { Federal Signal Corp. Model } \\
300 \text { GC, } 24 \text { VAC/OC Selectone } \\
\text { Connector Board: FS Corp. } \\
\text { Model } 300 \text { CK }\end{array}$ & $\begin{array}{l}H-2-28883 \text { sht } 1 \\
H-2-28883 \text { sht } 2 \\
H-2-28885 \text { sht } 8\end{array}$ \\
\hline $\mathrm{CAH}-21-3$ & $\begin{array}{l}\text { Criticality Atarm Horn. Located in } \\
\text { Building } 234-52 \text { Room } 116 \text {. }\end{array}$ & $\begin{array}{l}\text { Audible signaling Device: } \\
\text { Federal signal Corp. Model } \\
300 \text { GC, } 24 \text { VAC/DC Selectone } \\
\text { Connector Board: FS Corp. } \\
\text { Model } 300 \text { CK }\end{array}$ & $\begin{array}{l}H-2-28883 \text { sht } 1 \\
H-2-28883 \text { sht } 2 \\
H-2-28885 \text { sht } 8\end{array}$ \\
\hline $\mathrm{CAH}-\mathrm{Z} 1-4$ & $\begin{array}{l}\text { Criticality Alarm Horn. Located in } \\
\text { Building } 234-52 \text { Room } 221 \mathrm{E} \text {. }\end{array}$ & $\begin{array}{l}\text { Audible Signaling Device: } \\
\text { Federal signal Corp. Model } \\
300 \text { GC, } 24 \text { VAC/DC Selectone } \\
\text { Connector Board: FS Corp. } \\
\text { Model } 300 \text { CK }\end{array}$ & $\begin{array}{l}H-2-28883 \text { sht } 1 \\
H-2-28883 \text { sht } 2 \\
H-2-28885 \text { sht } 8\end{array}$ \\
\hline $\mathrm{CAH}-21-5$ & $\begin{array}{l}\text { Criticality Alarm Horn. Located in } \\
\text { Building } 234-5 Z \text { Corridor } 3 \text {. }\end{array}$ & $\begin{array}{l}\text { Audible Signaling Device: } \\
\text { Federal signal Corp. Model } \\
300 \text { GC, } 24 \text { VAC/DC Selectone } \\
\text { Connector Board: FS Corp. } \\
\text { Model } 300 \text { CK }\end{array}$ & $\begin{array}{l}H-2-28883 \text { sht } 1 \\
H-2-28883 \text { sht } 2 \\
H-2-28885 \text { sht } 8\end{array}$ \\
\hline $\mathrm{CAH}-\mathrm{Z1}-6$ & $\begin{array}{l}\text { Criticality Alarm Horn. Located in } \\
\text { Building } 234-5 Z \text { Corridor 242-8. }\end{array}$ & $\begin{array}{l}\text { Audible signaling Device: } \\
\text { Federal \$ignal Corp. Model } \\
300 \text { GC, } 24 \text { VAC/DC Selectone } \\
\text { Connector Board: FS Corp. } \\
\text { Model } 300 \text { CK }\end{array}$ & $\begin{array}{l}H-2-28883 \text { sht } 1 \\
H-2-28883 \text { sht } 2 \\
H-2-28885 \text { sht } 8\end{array}$ \\
\hline $\mathrm{CAH}-\mathrm{Z1}-7$ & $\begin{array}{l}\text { Criticality Alarm Horn. Located in } \\
\text { Building } 234-52 \text { Corridor } 4 \text {. }\end{array}$ & $\begin{array}{l}\text { Audible signaling Device: } \\
\text { Federal Signal Corp. Model } \\
300 \text { GC, } 24 \text { VAC/DC Sel ectone } \\
\text { Connector Board: FS Corp. } \\
\text { Model } 300 \text { CK }\end{array}$ & $\begin{array}{l}H-2-28883 \text { sht } 1 \\
H-2-28883 \text { sht } 2 \\
H-2-28885 \text { sht } 8\end{array}$ \\
\hline $\mathrm{CAH}-\mathrm{Z} 1-8$ & $\begin{array}{l}\text { Criticality Alarm Horn. Located in } \\
\text { Building } 234-52 \text { Corridor } 4 \mathrm{C} \text {. }\end{array}$ & $\begin{array}{l}\text { Audible Signaling Device: } \\
\text { Federal Signal Corp. Model } \\
300 \text { GC, } 24 \text { VAC/DC Selectone } \\
\text { Connector Board: FS Corp. } \\
\text { Model } 300 \text { CK }\end{array}$ & $\begin{array}{l}H-2-28883 \text { sht } 1 \\
H-2-28883 \text { sht } 2 \\
H-2-28885 \text { sht } 8\end{array}$ \\
\hline CAH-Z1-9 & Criticality Alarm Horn. Located in 242-z. & $\begin{array}{l}\text { Audible signaling Device: } \\
\text { Federal signal Corp. Model } \\
300 \text { GC, } 24 \text { VAC/DC Selectone } \\
\text { Connector Board: FS Corp. } \\
\text { Model } 300 \text { CK } \\
\end{array}$ & $\begin{array}{l}H-2-28883 \text { sht } 1 \\
H-2-28883 \text { sht } 2 \\
H-2-28885 \text { sht } 8\end{array}$ \\
\hline CAH $-Z 1-10$ & $\begin{array}{l}\text { Criticality Alarm Horn. Located in } \\
\text { Building } 234-52 \text { Room } 137 \text {. }\end{array}$ & $\begin{array}{l}\text { Audible Signaling Device: } \\
\text { Federal sjgnal Corp. Model } \\
300 \text { GC, } 24 \text { VAC/DC Select one } \\
\text { Connector Board: FS corp. } \\
\text { Model } 300 \text { CK }\end{array}$ & $\begin{array}{l}H-2-28883 \text { sht } 1 \\
H-2-28883 \text { sht } 2 \\
H-2-28885 \text { sht } 8\end{array}$ \\
\hline
\end{tabular}




\begin{tabular}{|c|c|c|c|}
\hline 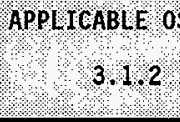 & 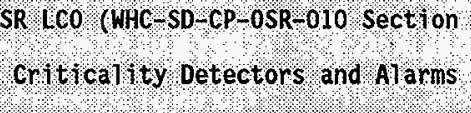 & $\begin{array}{l}301 \\
3\end{array}$ & \\
\hline 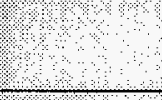 & 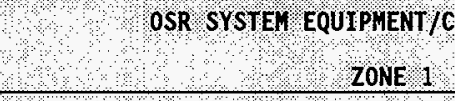 & ONENT UST & \\
\hline $\begin{array}{l}\text { MASTER } \\
\text { COMPONENT } \\
\text { NUNDEX } \\
\text { NUBER }\end{array}$ & OESTCRIPIIONA & $\begin{array}{l}\text { MANUFACTURER } \\
\text { PART/MODEL } \\
\text { NUNBER } \\
\text { (DWG } \mathrm{H}-2-28884 \text { ) }\end{array}$ & $\begin{array}{l}\text { ESSEWIAL } \\
\text { DRAWWG } \\
\text { NUMBER }\end{array}$ \\
\hline CAH-Z1-12 & Criticality Alarm Horn. Located in $242-z$. & $\begin{array}{l}\text { Audible signaling Device: } \\
\text { Federal Signal Corp. Model } \\
300 \text { GC, } 24 \text { VAC/DC Selectone } \\
\text { Connector Board: FS Corp. } \\
\text { Model } 300 \text { CK }\end{array}$ & $\begin{array}{l}H-2-28883 \text { sht } 1 \\
H-2-28883 \text { sht } 2 \\
H-2-28885 \text { sht } 8\end{array}$ \\
\hline CAK-21-13 & $\begin{array}{l}\text { Criticality Alarm Horn. Located outside on } \\
\text { East wall of } 234-52 \text {. }\end{array}$ & $\begin{array}{l}\text { Audible Signal ing Device: } \\
\text { Federal Signal Corp. Model } \\
300 \mathrm{GC}, 24 \text { VAC/DC Selectone } \\
\text { Connector Board: FS Corp. } \\
\text { Model } 300 \text { CK }\end{array}$ & $\begin{array}{l}H-2-28883 \text { sht } 1 \\
H-2-28883 \text { sht } 2 \\
H-2-28885 \text { sht } 8\end{array}$ \\
\hline CAH-Z1-14 & $\begin{array}{l}\text { Criticality Alarm Horn. Located outside on. } \\
\text { South wall of } 234-5 z \text {. }\end{array}$ & $\begin{array}{l}\text { Audible signal ing Device: } \\
\text { Federal Signal Corp. Model } \\
300 \text { GC, } 24 \text { VAC/DC Selectone } \\
\text { Connector Board: FS Corp. } \\
\text { Model } 300 \text { CK }\end{array}$ & $\begin{array}{l}H-2-28883 \text { sht } 1 \\
H-2-28883 \text { sht } 2 \\
H-2-28885 \text { sht } 8\end{array}$ \\
\hline CAH-21-16 & $\begin{array}{l}\text { Criticality Alarm Horn. Located outside on } \\
\text { North wall of } 234-5 z \text {. }\end{array}$ & $\begin{array}{l}\text { Audible signal ing Device: } \\
\text { Federal Signal Corp. Model } \\
300 \text { GC, } 24 \text { VAC/DC Selectone } \\
\text { Connector Board: FS Corp. } \\
\text { Model } 300 \text { CK }\end{array}$ & $\begin{array}{l}H-2-28883 \text { sht } 1 \\
H-2-28883 \text { sht } 2 \\
H-2-28885 \text { sht } 8\end{array}$ \\
\hline $\mathrm{CAH}-\mathrm{Z1}-17$ & $\begin{array}{l}\text { Criticality Alarm Horn. Located in } \\
\text { Building } 234-52 \text { Room } 132 \text {. }\end{array}$ & $\begin{array}{l}\text { Audible signal ing Device: } \\
\text { Federal Signal Corp. Model } \\
300 \text { GC, } 24 \text { VAC/DC Selectone } \\
\text { Connector Board: FS Corp. } \\
\text { Model } 300 \text { CK }\end{array}$ & $\begin{array}{l}H-2-28883 \text { sht } 1 \\
H-2-28883 \text { sht } 2 \\
H-2-28885 \text { sht } 8\end{array}$ \\
\hline $\mathrm{CAH}-21-18$ & $\begin{array}{l}\text { Criticality Alarm Horn. Located in } \\
\text { Building } 234-52 \text { Room 221D. }\end{array}$ & $\begin{array}{l}\text { Audible signaling Device: } \\
\text { Federal signal Corp. Model } \\
300 \text { GC, } 24 \text { VAC/DC Selectone } \\
\text { Connector Board: FS Corp. } \\
\text { Model } 300 \text { CK }\end{array}$ & $\begin{array}{l}H-2-28883 \text { sht } 1 \\
H-2-28883 \text { sht } 2 \\
H-2-28885 \text { sht } 8\end{array}$ \\
\hline CAH-Z1- 19 & $\begin{array}{l}\text { Criticality Alarm Horn. Located in } \\
\text { Building } 234-52 \text { Room } 221 \text {. }\end{array}$ & $\begin{array}{l}\text { Audible signaling Device: } \\
\text { Federal Signal Corp. Model } \\
300 \text { GC, } 24 \text { VAC/DC Selectone } \\
\text { Connector Board: FS Corp. } \\
\text { Model } 300 \text { CX }\end{array}$ & $\begin{array}{l}\text { H-2-28883 sht ? } \\
\text { H-2-28883 sht } 2 \\
\text { H-2-28885 sht } 8\end{array}$ \\
\hline
\end{tabular}




\begin{tabular}{|c|c|c|c|}
\hline \multicolumn{4}{|c|}{ 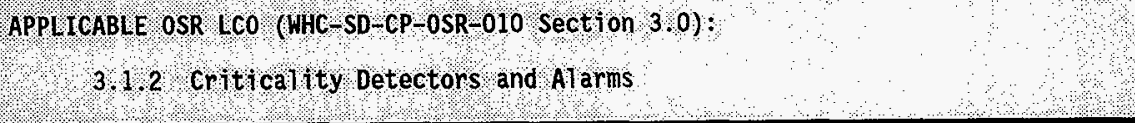 } \\
\hline & $\begin{array}{l}\text { 0SR SYSTEM EQUIPHENT / } \\
\text { 20NE } 1\end{array}$ & ONENT LIST & \\
\hline $\begin{array}{l}\text { MASTER } \\
\text { COMPONENT } \\
\text { NONDEX } \\
\text { NUMBER }\end{array}$ & DELSCRTPTION & $\begin{array}{l}\text { WANUFACTURER } \\
\text { PART /MODEL } \\
\text { NUMBER } \\
\text { (DWG } H=2-28884)\end{array}$ & $\begin{array}{l}\text { ESSENAHA } \\
\text { DRAWING } \\
\text { NUNBER }\end{array}$ \\
\hline CAH- $21-20$ & $\begin{array}{l}\text { Criticality Alarm Horn. Located in } \\
\text { Building } 234-52 \text { Room } 2218 \text {. }\end{array}$ & $\begin{array}{l}\text { Audible Signal ing Device: } \\
\text { Federal Signal Corp. Model } \\
300 \mathrm{GC}, 24 \text { VAC/DC Selectone } \\
\text { Connector Board: FS Corp. } \\
\text { Model } 300 \text { CK }\end{array}$ & $\begin{array}{l}H-2-28883 \text { sht } 1 \\
H-2-28883 \text { sht } 2 \\
H-2-28885 \text { sht } 8\end{array}$ \\
\hline CAH-21-21 & $\begin{array}{l}\text { Criticality Alarm Horn. Located on } \\
\text { Northeast Roof of } 234-52 \text {. }\end{array}$ & $\begin{array}{l}\text { Audible signal ing Device: } \\
\text { Federal Signal Corp. Model } \\
300 \mathrm{GC}, 24 \text { VAC/DC Selectone } \\
\text { Connector Board: FS Corp. } \\
\text { Model } 300 \text { CK }\end{array}$ & $\begin{array}{l}\text { H-2-28883 sht } 1 \\
H-2-28883 \text { sht } 2 \\
H-2-28885 \text { sht } 11\end{array}$ \\
\hline $\mathrm{CAH}-\mathrm{Z1}-22$ & $\begin{array}{l}\text { Criticality Alarm Horn. Located on North } \\
\text { Roof of } 234-5 z \text {. }\end{array}$ & $\begin{array}{l}\text { Audible Signaling Device: } \\
\text { Federal signal Corp. Model } \\
300 \mathrm{GC}, 24 \text { VAC/DC Selectone } \\
\text { Connector Board: FS Corp. } \\
\text { Model } 300 \text { CK }\end{array}$ & $\begin{array}{l}H-2-28883 \text { sht } 1 \\
H-2-28883 \text { sht } 2 \\
H-2-28885 \text { sht } 11\end{array}$ \\
\hline САН-21-23 & $\begin{array}{l}\text { Criticality Alarm Horn. Located on South } \\
\text { Roof of } 234-5 z \text {. }\end{array}$ & $\begin{array}{l}\text { Audible signaling Device: } \\
\text { Federal Signal Corp. Hodel } \\
300 \text { GC, } 24 \text { VAC/DC Selectone } \\
\text { Connector Board: FS Corp. } \\
\text { Model } 300 \text { CK }\end{array}$ & $\begin{array}{l}H-2-28883 \text { sht } 1 \\
H-2-28883 \text { sht } 2 \\
H-2-28885 \text { sht } 11\end{array}$ \\
\hline CALM-Z1-1 & $\begin{array}{l}\text { Criticality Alarm Logic Module. Provides } \\
\text { the logic circuitry for annunciation of } \\
\text { alert and alarm conditions as detected by } \\
\text { the CADMs. Located in CAP-21. }\end{array}$ & PER Orawing $H-2-91179$ & $\begin{array}{l}H-2-28883 \text { sht } 1 \\
H-2-28883 \text { sht } 2 \\
H=2-28885 \text { sht } 8\end{array}$ \\
\hline CALM-21-2 & $\begin{array}{l}\text { Criticality Alarm Logic Module. Provides } \\
\text { the logic circuitry for annunciation of } \\
\text { alert and alarm conditions as detected by } \\
\text { the CADMs. Located in CAP-21. }\end{array}$ & PER Orawing $\mathrm{H}-2-91179$ & $\begin{array}{l}H-2-28883 \text { sht } 1 \\
H-2-28883 \text { sht } 2 \\
H-2-28885 \text { sht } 8\end{array}$ \\
\hline CALM-21-3 & $\begin{array}{l}\text { Criticality Alarm Logic Module. Provides } \\
\text { the logic circuitry for annunciation of } \\
\text { alert and alarm conditions as detected by } \\
\text { the CADMs. Located in CAP- } 21 \text {. }\end{array}$ & Per Drawing $\mathrm{H}-2-91179$ & $\begin{array}{l}H-2-28883 \text { sht } 1 \\
H-2-28883 \text { sht } 2 \\
H-2-28885 \text { sht } 8\end{array}$ \\
\hline CAP $-Z 1$ & $\begin{array}{l}\text { Criticality Alarm Panel zone } 1 \text {. Contains } \\
\text { the CADMs, CALMs, CARM, Inhibit switches, } \\
\text { local (building) annunciation and Intertie } \\
\text { relays for the Zone } 1 \text { Criticality Alarm } \\
\text { System. Located in Building } 234-52 \\
\text { Corridor 2. }\end{array}$ & $\begin{array}{l}\text { Relay Rack. } \\
\text { Premier, TVA } 7719-23\end{array}$ & $\begin{array}{l}H-2-28883 \text { sht } 1 \\
H-2-28883 \text { sht } 2 \\
H-2-28885 \text { sht } 8\end{array}$ \\
\hline CARM-Z1 & $\begin{array}{l}\text { Criticality Alarm Relay Module. Activates } \\
\text { the alarm signal generator and provides an } \\
\text { annunciation signal in the event of a high } \\
\text { alarm signal from the CALM. Includes an } \\
\text { inhibit switch to disable the alarm output } \\
\text { signal. Located in CAP-Z1. }\end{array}$ & Per Drawing $\mathrm{H}-2-91180$ & $\begin{array}{l}H-2-28883 \text { sht } 1 \\
H-2-28883 \text { sht } 2 \\
H-2-28885 \text { sht } 8\end{array}$ \\
\hline
\end{tabular}




\begin{tabular}{|c|c|c|c|}
\hline \multicolumn{4}{|c|}{ 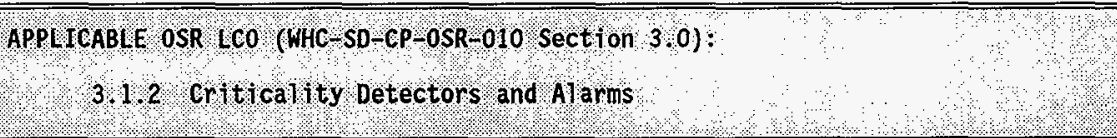 } \\
\hline 189 & oOSR SYSTEV EQUTPMENT/ & PONENT LIST & \\
\hline $\begin{array}{l}\text { WASTER } \\
\text { COMPONENT } \\
\text { NUMBER }\end{array}$ & DENCSTONAL & $\begin{array}{l}\text { MANUFACTURER } \\
\text { PART/HODEL } \\
\text { NUWBER } \\
\text { (DWG }-2,28884 \text { ) }\end{array}$ & $\begin{array}{l}\text { ESSEWIM AL } \\
\text { DRAWTWG } \\
\text { NUWBER }\end{array}$ \\
\hline $\begin{array}{l}\text { AC POWER RELAY } \\
\text { (Not required } \\
\text { for labeling } \\
\text { at this time.) } \\
\end{array}$ & $\begin{array}{l}\text { (SC-3 SE). AC Power Monitor Relay. } \\
\text { Deenergizes on loss of power. A manual } \\
\text { reset is requi red to prevent a fluctuating } \\
\text { (errat ic) power supply from initiating } \\
\text { false alarms. }\end{array}$ & Allen-Bradley $\# 700-C 201 \mathrm{~A} 1$ & $\begin{array}{l}H-2-28883 \text { sht } 1 \\
H-2-28883 \text { sht } 2 \\
H-2-28885 \text { sht } 8\end{array}$ \\
\hline$s-1-1 A$ & $\begin{array}{l}\text { Inhibit Switch for CAD/CADM-21-1A used to } \\
\text { prevent annunciation of alarm during test, } \\
\text { cal ibration, and replacement of CADS and } \\
\text { CADMs. Located on CAP-Z1. }\end{array}$ & $\begin{array}{l}\text { Square D Class } 9001, \\
\text { Type LRSA-2K1 with } \\
\text { Type LA-1 Contact Blocks }\end{array}$ & $\begin{array}{l}H-2-28883 \text { sht } 1 \\
H-2-28883 \text { sht } 2 \\
H-2-28885 \text { sht } 8\end{array}$ \\
\hline$s-1-1 c$ & $\begin{array}{l}\text { Inhibit Switch for CAD/CADM-Z1-1C used to } \\
\text { prevent annunciation of alarm during test, } \\
\text { cal ibration, and replacement of CADS and } \\
\text { CADMs. Located on CAP-Z1. }\end{array}$ & $\begin{array}{l}\text { Square D Class } 9001 \\
\text { Type LRSA-2K1 with } \\
\text { Type LA-1 Contact Blocks }\end{array}$ & $\begin{array}{l}H-2-28883 \text { sht } 1 \\
H-2-28883 \text { sht } 2 \\
H-2-28885 \text { sht } 8\end{array}$ \\
\hline$s-1-2 A$ & $\begin{array}{l}\text { Inhibit Switch for CAD/CADM-Z1-2A used to } \\
\text { prevent annunciation of alarm during test, } \\
\text { cal ibration, and replacement of CADS and } \\
\text { CADMs. Located on CAP-Z1. }\end{array}$ & $\begin{array}{l}\text { Square D Class } 9001, \\
\text { Type LRSA-2K1 with } \\
\text { Type LA-1 Contact Blocks }\end{array}$ & $\begin{array}{l}H-2-28883 \text { sht } 1 \\
H-2-28883 \text { sht } 2 \\
H-2-28885 \text { sht } 8\end{array}$ \\
\hline$s-1-2 B$ & $\begin{array}{l}\text { Inhibit Switch for CAD/CADM-Z1-2B used to } \\
\text { prevent annunciation of alarm during test, } \\
\text { calibration, and replacement of CADS and } \\
\text { CADMs. Located on CAP-Z1. }\end{array}$ & $\begin{array}{l}\text { Square D Class } 9001 \\
\text { Type LRSA-2K1 with } \\
\text { Type LA-1 Contact Blocks }\end{array}$ & $\begin{array}{l}H-2-28883 \text { sht } 1 \\
H-2-28883 \text { sht } 2 \\
H-2-28885 \text { sht } 8\end{array}$ \\
\hline$s-1-2 c$ & $\begin{array}{l}\text { Inhibit switch for CAD/CADM- } 21-2 C \text { used to } \\
\text { prevent annunciation of alarm during test, } \\
\text { cal ibration, and replacement of CADs and } \\
\text { CADMs. Located on CAP-Z1. }\end{array}$ & $\begin{array}{l}\text { Square D Class } 9001, \\
\text { Type LRSA-2K1 with } \\
\text { Type LA- } 1 \text { Contact Blocks }\end{array}$ & $\begin{array}{l}\text { H-2-28883 sht } 1 \\
\text { H-2-28883 sht } 2 \\
\text { H-2-28885 sht } 8\end{array}$ \\
\hline
\end{tabular}




\begin{tabular}{lr} 
DEFINITION AND MEANS OF MAINTAINING THE & HNF-SD-CP-SDD-003 \\
CRITICALITY DETECTORS AND ALARMS PORTION & Rev. 4 \\
OF THE PFP SAFETY ENVELOPE & Page 24 of 94 \\
\hline
\end{tabular}

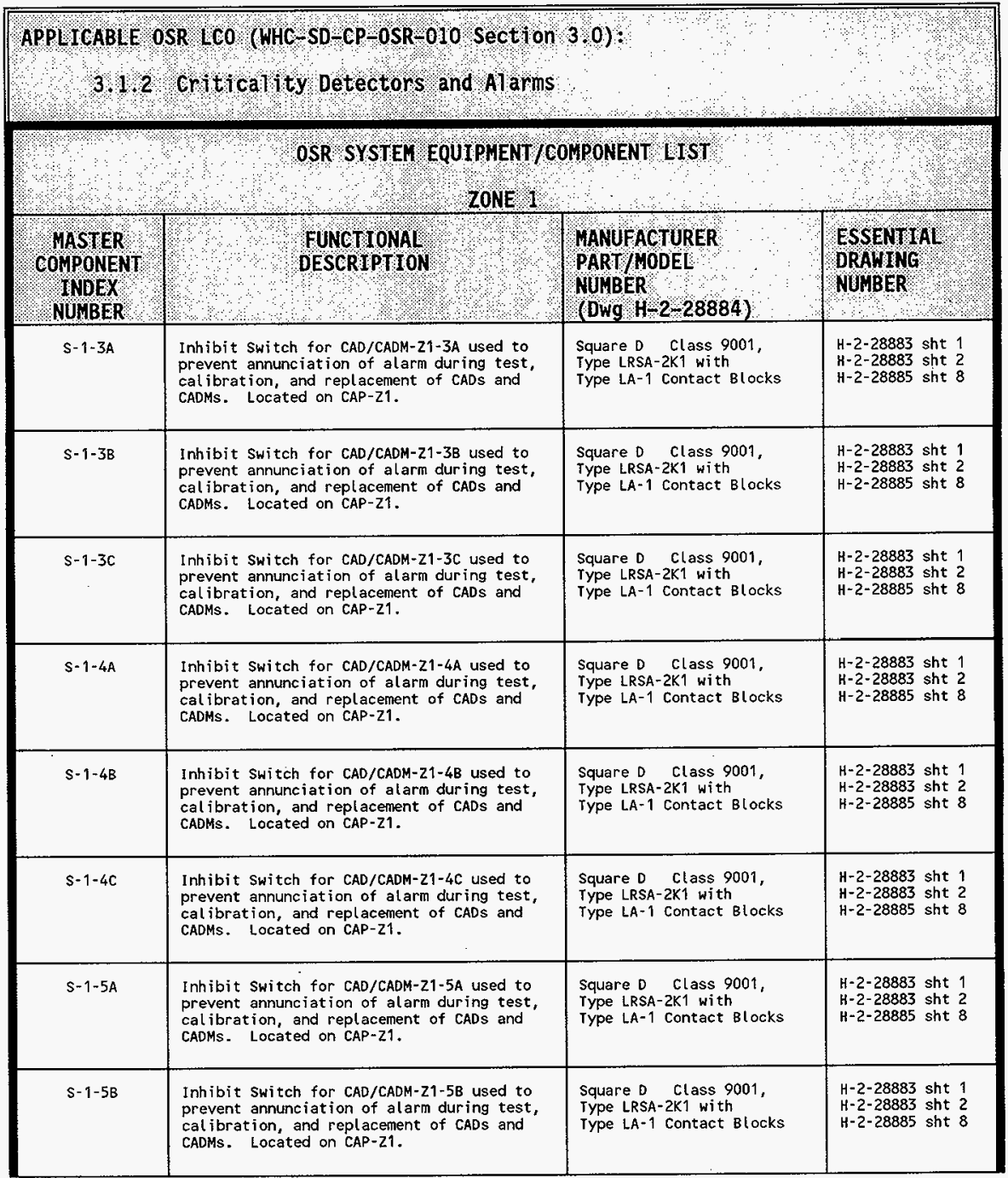




\begin{tabular}{|c|c|c|c|}
\hline \multicolumn{4}{|c|}{ 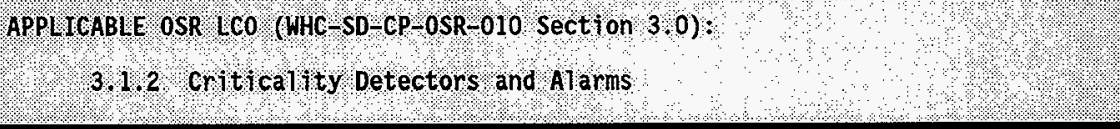 } \\
\hline 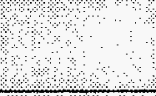 & $\begin{array}{r}\text { OSR SYSTEN EQUTPHENT } \\
\qquad \mathrm{ZONE} 1 \\
\end{array}$ & PONENT LIST & \\
\hline $\begin{array}{l}\text { MASTER } \\
\text { COMPONENT } \\
\text { NUNDEX } \\
\text { NUMBER }\end{array}$ & 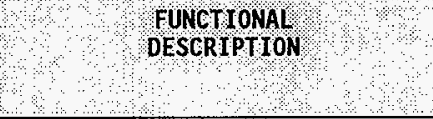 & $\begin{array}{l}\text { MANUFACTURER } \\
\text { PART/WODEL } \\
\text { NUNBER } \\
\text { (DWg } \mathrm{H}-2-28884 \text { ) }\end{array}$ & $\begin{array}{l}\text { ESSENTIAL } \\
\text { DRAHING } \\
\text { NUMBER }\end{array}$ \\
\hline$s-1-5 c$ & $\begin{array}{l}\text { Inhibit Switch for CAD/CADM- }-21-5 C \text { used to } \\
\text { prevent annunciation of alarm during test, } \\
\text { calibration, and replacement of CADS and } \\
\text { CADMs. Located on CAP- } 21 \text {. }\end{array}$ & $\begin{array}{l}\text { Square } D \text { Class } 9001, \\
\text { Type LRSA-2K1 with } \\
\text { Type LA-1 Contact Blocks }\end{array}$ & $\begin{array}{l}H-2-28883 \text { sht } 1 \\
H-2-28883 \text { sht } 2 \\
H-2-28885 \text { sht } 8\end{array}$ \\
\hline$s-1-6 A$ & $\begin{array}{l}\text { Inhibit Switch for CAD/CADM-Z1-6A used to } \\
\text { prevent annunciation of alarm during test, } \\
\text { calibration, and replacentent of CADS and } \\
\text { CADMs. Located on CAP-Z1. }\end{array}$ & $\begin{array}{l}\text { Square } D \text { Class } 9001 \text {, } \\
\text { Type LRSA-2K1 with } \\
\text { Type LA-1 Contact Blocks }\end{array}$ & $\begin{array}{l}H-2-28883 \text { sht } 1 \\
H-2-28883 \text { sht } 2 \\
H-2-28885 \text { sht } 8\end{array}$ \\
\hline$s-1-6 B$ & $\begin{array}{l}\text { Inhibit Switch for CAD/CADM-21-6B used to } \\
\text { prevent annunciat ion of alarm during test, } \\
\text { calibration, and replacement of CADs and } \\
\text { CADMs. Located on CAP-21. }\end{array}$ & $\begin{array}{l}\text { Square D Class } 9001 \text {, } \\
\text { Type LRSA-2K1 with } \\
\text { Type LA-1 Contact Blocks }\end{array}$ & $\begin{array}{l}H-2-28883 \text { sht } 1 \\
H-2-28883 \text { sht } 2 \\
H-2-28885 \text { sht } 8\end{array}$ \\
\hline$s-1-6 c$ & $\begin{array}{l}\text { Inhibit Switch for CAD/CADM-Z1-6C used to } \\
\text { prevent annunciat ion of alarm during test, } \\
\text { cal ibration, and replacement of CADS and } \\
\text { CADMs. Located on CAP-Z1. }\end{array}$ & $\begin{array}{l}\text { Square D Class } 9001 \text {, } \\
\text { Type LRSA-2K1 with } \\
\text { Type LA-1 Contact Blocks }\end{array}$ & $\begin{array}{l}H-2-28883 \text { sht } 1 \\
H-2-28883 \text { sht } 2 \\
H-2-28885 \text { sht } 8\end{array}$ \\
\hline
\end{tabular}




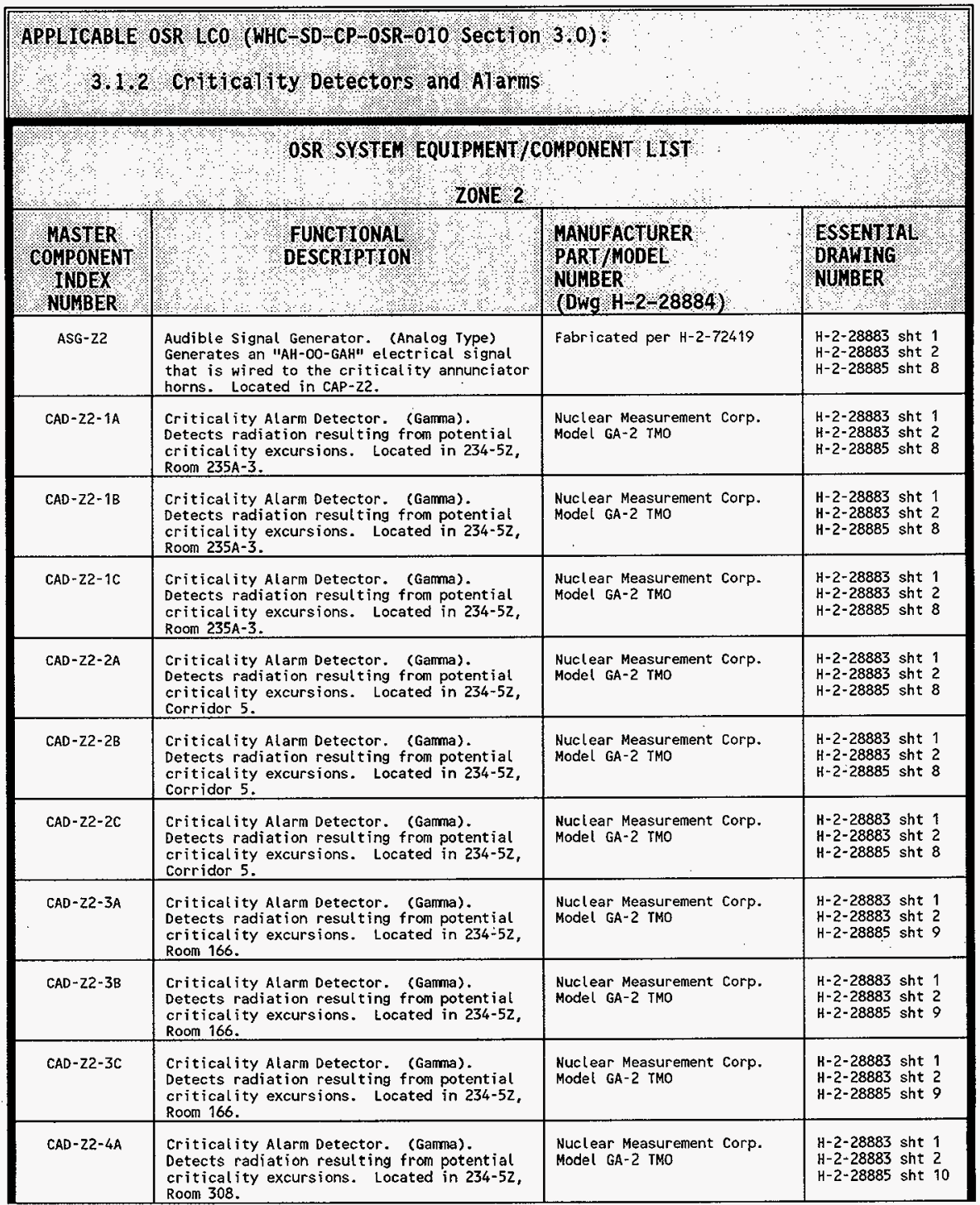




\begin{tabular}{|c|c|c|c|}
\hline \multicolumn{4}{|c|}{ 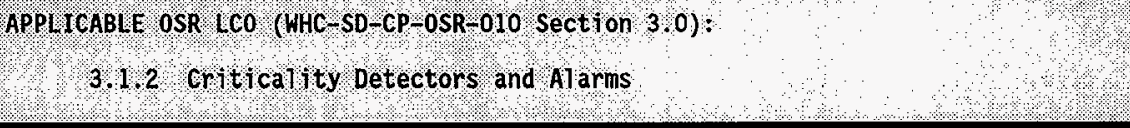 } \\
\hline 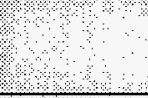 & 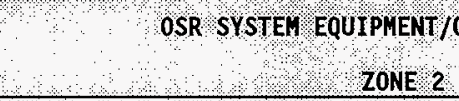 & PONENT LIST & \\
\hline $\begin{array}{l}\text { WASTER } \\
\text { COMPONENT } \\
\text { NWDEX, } \\
\text { NUMBER }\end{array}$ & 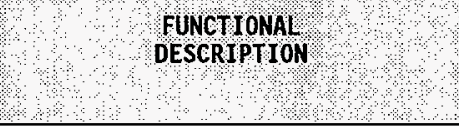 & $\begin{array}{l}\text { WANUFACTURER } \\
\text { PART } \\
\text { (DWBOEEL } \\
\text { (DWG } 1-2-28884 \text { ) }\end{array}$ & 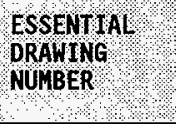 \\
\hline$C A D-22-4 C$ & $\begin{array}{l}\text { Criticality Alarm Detector. (Gamma). } \\
\text { Detects radiation resulting from potential } \\
\text { criticality excursions. Located in } 234-52 \text {, } \\
\text { Room } 308 \text {. }\end{array}$ & $\begin{array}{l}\text { Nuclear Measurement Corp. } \\
\text { Model GA-2 TMO }\end{array}$ & $\begin{array}{l}H-2-28883 \text { sht } 1 \\
H-2-28883 \text { sht } 2 \\
H-2-28885 \text { sht } 10\end{array}$ \\
\hline CADM-Z2-1A & $\begin{array}{l}\text { Critical ity Alarm Detection Module. One (1) } \\
\text { per CAD. Provides dose rate indication, } \\
\text { calibration adjustments for the CAD-CADM } \\
\text { unit, and alarm functions. Located in } \\
\text { CAP-Z2. }\end{array}$ & $\begin{array}{l}\text { Nuclear Measurement Corp. } \\
\text { Model GA-2 TMO }\end{array}$ & $\begin{array}{l}H-2-28883 \text { sht } 1 \\
H-2-28883 \text { sht } 2 \\
H-2-28885 \text { sht } 8\end{array}$ \\
\hline CADM-Z2-1B & $\begin{array}{l}\text { Criticality Alarm Detection Module. One ( } 1 \text { ) } \\
\text { per CAD. Provides dose rate indication, } \\
\text { calibration adjustments for the CAD-CADM } \\
\text { unit, and alarm functions. Located in } \\
\text { CAP-Z2. }\end{array}$ & $\begin{array}{l}\text { Nuclear Measurement Corp. } \\
\text { Model GA-2 TMO }\end{array}$ & $\begin{array}{l}H-2-28883 \text { sht } 1 \\
H-2-28883 \text { sht } 2 \\
H-2-28885 \text { sht } 8\end{array}$ \\
\hline CADM-22-2B & $\begin{array}{l}\text { Criticality Alarm Detection Module. One (1) } \\
\text { per CAD. Provides dose rate indication, } \\
\text { calibration adjustments for the CAD-CADM } \\
\text { unit, and alarm functions. Located in } \\
\text { CAP-Z2. }\end{array}$ & $\begin{array}{l}\text { Nuclear Measurement Corp. } \\
\text { Model GA-2 TMO }\end{array}$ & $\begin{array}{l}\text { H-2-28883 sht } 1 \\
\text { H-2-28883 sht } 2 \\
\text { H-2-28885 sht } 8\end{array}$ \\
\hline CADH- $-22-2 C$ & $\begin{array}{l}\text { Criticality Alarm Detection Module. One (1) } \\
\text { per CAD. Provides dose rate indication, } \\
\text { calibration adjustments for the CAD-CADM } \\
\text { unit, and alarm functions. Located in } \\
\text { CAP-Z2. }\end{array}$ & $\begin{array}{l}\text { Nuclear Measurement Corp. } \\
\text { Model GA-2 TMO }\end{array}$ & $\begin{array}{l}H-2-28883 \text { sht } 1 \\
H-2-28883 \text { sht } 2 \\
H-2-28885 \text { sht } 8\end{array}$ \\
\hline$C A D M-22-3 A$ & $\begin{array}{l}\text { Critical ity Alarm Detection Module. One (1) } \\
\text { per CAD. Provides dose rate indication, } \\
\text { calibration adjustments for the CAD-CADM } \\
\text { unit, and alarm functions. Located in } \\
\text { CAP-Z2. }\end{array}$ & $\begin{array}{l}\text { Nuclear Measurement Corp. } \\
\text { Model GA-2 TMO }\end{array}$ & $\begin{array}{l}H-2-28883 \text { sht } 1 \\
H-2-28883 \text { sht } 2 \\
H-2-28885 \text { sht } 8\end{array}$ \\
\hline
\end{tabular}




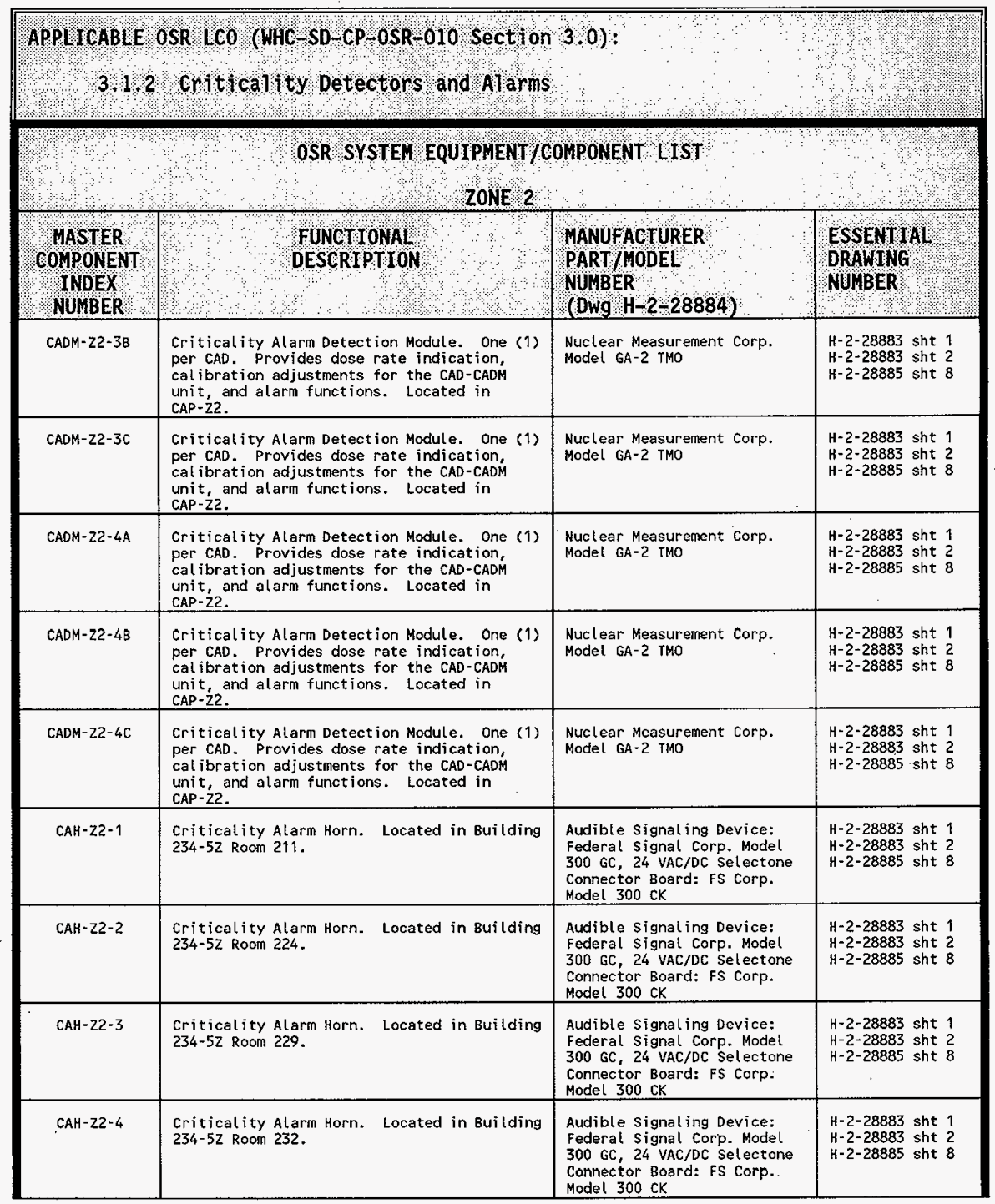




\begin{tabular}{|c|c|c|c|}
\hline APPLICABLE & 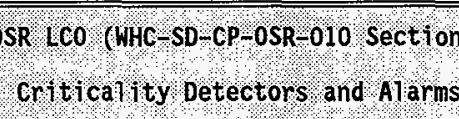 & $3.0): \quad$ & ons? \\
\hline 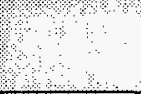 & $\begin{array}{l}\text { OSR SYSTEM EQUTPNENT/C } \\
\text { ZONE 2 }\end{array}$ & MPONENT LIST & \\
\hline $\begin{array}{l}\text { TASTER } \\
\text { QONPONENT } \\
\text { NWDEX } \\
\text { NUMBER }\end{array}$ & FUNCTIONAL & $\begin{array}{l}\text { WANUFACTURER } \\
\text { PART/WODEL } \\
\text { NUMBER } \\
(D W G H 2-28884)\end{array}$ & $\begin{array}{l}\text { ESSENTHL } \\
\text { DRAWTMG } \\
\text { NOMBER }\end{array}$ \\
\hline $\mathrm{CAH}-22-5$ & $\begin{array}{l}\text { Criticality Alarm Horn. Located in Building } \\
234-52 \text { Room } 233 \text {. }\end{array}$ & $\begin{array}{l}\text { Audible Signaling Device: } \\
\text { Federal Signal Corp. Model } \\
300 \mathrm{GC}, 24 \text { VAC/DC Selectone } \\
\text { Connector Board: FS Corp. } \\
\text { Model } 300 \text { CK }\end{array}$ & $\begin{array}{l}H-2-28883 \text { sht } 1 \\
H-2-28883 \text { sht } 2 \\
H-2-28885 \text { sht } 8\end{array}$ \\
\hline $\mathrm{CAH}-22-6$ & $\begin{array}{l}\text { Criticality Alarm Horn. Located in Building } \\
234-52 \text { Corridor } 5 \text {. }\end{array}$ & $\begin{array}{l}\text { Audible signaling Device: } \\
\text { Federal signal Corp. Model } \\
300 \mathrm{GC}, 24 \text { VAC/DC Selectone } \\
\text { Connector Board: FS Corp. } \\
\text { Model } 300 \text { CK }\end{array}$ & $\begin{array}{l}H-2-28883 \text { sht } 1 \\
H-2-28883 \text { sht } 2 \\
H-2-28885 \text { sht } 8\end{array}$ \\
\hline $\mathrm{CAH}-\mathrm{Z2}-7$ & $\begin{array}{l}\text { Criticality Alarm Horn. Located outside on } \\
\text { South wall of Building } 234-5 \mathrm{Z} \text {. }\end{array}$ & $\begin{array}{l}\text { Audible Signaling Device: } \\
\text { Federal Signal Corp. Model } \\
300 \text { GC, } 24 \text { VAC/OC Selectone } \\
\text { Connector Board: FS Corp. } \\
\text { Model } 300 \text { CK }\end{array}$ & $\begin{array}{l}H-2-28883 \text { sht } 1 \\
H-2-28883 \text { sht } 2 \\
H-2-28885 \text { sht } 8\end{array}$ \\
\hline $\mathrm{CAH}-22-8$ & $\begin{array}{l}\text { Criticality Alarm Horn. Located in Building } \\
234-52 \text { Room } 262 \text {. }\end{array}$ & $\begin{array}{l}\text { Audible signaling Device: } \\
\text { Federal signal Corp. Model } \\
300 \text { GC, } 24 \text { VAC/DC Selectone } \\
\text { Connector Board: FS Corp. } \\
\text { Model } 300 \text { CK }\end{array}$ & $\begin{array}{l}H-2-28883 \text { sht } 1 \\
H-2-28883 \text { sht } 2 \\
H-2-28885 \text { sht } 9\end{array}$ \\
\hline $\mathrm{CAH}-22-9$ & $\begin{array}{l}\text { Criticality Alarm Horn. Located in Building } \\
234-52 \text { Room } 262 \text {. }\end{array}$ & $\begin{array}{l}\text { Audible Signaling Device: } \\
\text { Federal Signal Corp. Model } \\
300 \mathrm{GC}, 24 \text { VAC/DC Selectone } \\
\text { Connector Board: FS Corp. } \\
\text { Model } 300 \text { CK }\end{array}$ & $\begin{array}{l}H-2-28883 \text { sht } 1 \\
H-2-28883 \text { sht } 2 \\
H-2-28885 \text { sht } 9\end{array}$ \\
\hline САН-22-10 & $\begin{array}{l}\text { Criticality Alarm Horn. Located in } \\
\text { Building } 234-52 \text { Room } 262 \text {. }\end{array}$ & $\begin{array}{l}\text { Audible Signaling Device: } \\
\text { Federal Signal Corp. Model } \\
300 \text { GC, } 24 \text { VAC/OC Selectone } \\
\text { Connector Board: FS Corp. } \\
\text { Model } 300 \text { CK }\end{array}$ & $\begin{array}{l}H-2-28883 \text { sht } 1 \\
H-2-28883 \text { sht } 2 \\
H-2-28885 \text { sht } 9 .\end{array}$ \\
\hline CAH-Z2-11 & $\begin{array}{l}\text { Criticality Alarm Horn. Located in } \\
\text { Building } 234-52 \text { Room } 260 \text {. }\end{array}$ & $\begin{array}{l}\text { Audible Signaling Device: } \\
\text { Federal Signal Corp. Model } \\
300 \text { GC, } 24 \text { VAC/DC Selectone } \\
\text { Connector Board: FS Corp. } \\
\text { Model } 300 \text { CK }\end{array}$ & $\begin{array}{l}H-2-28883 \text { sht } 1 \\
H-2-28883 \text { sht } 2 \\
H-2-28885 \text { sht } 9\end{array}$ \\
\hline CAH-Z2-12 & $\begin{array}{l}\text { Criticality Alarm Horn. Located in Building } \\
234-52 \text { Room } 263 \text {. }\end{array}$ & $\begin{array}{l}\text { Audible Signaling Device: } \\
\text { Federal Signal Corp. Model } \\
300 \text { GC, } 24 \text { VAC/DC Selectone } \\
\text { Connector Board: FS Corp. } \\
\text { Model } 300 \text { CK }\end{array}$ & $\begin{array}{l}\text { H-2-28883 sht } 1 \\
H-2-28883 \text { sht } 2 \\
H-2-28885 \text { sht } 9\end{array}$ \\
\hline CALH-Z2-13 & $\begin{array}{l}\text { Criticality Alarm Horn. Located in Building } \\
234-52 \text { Room } 260 \text {. }\end{array}$ & $\begin{array}{l}\text { Audible Signaling Device: } \\
\text { Federal signal Corp. Model } \\
300 \text { GC, } 24 \text { VAC/DC Selectone } \\
\text { Connector Board: FS Corp. } \\
\text { Model } 300 \text { CK }\end{array}$ & $\begin{array}{l}\text { H-2-28883 sht } 1 \\
H-2-28883 \text { sht } 2 \\
H-2-28885 \text { sht } 9\end{array}$ \\
\hline
\end{tabular}




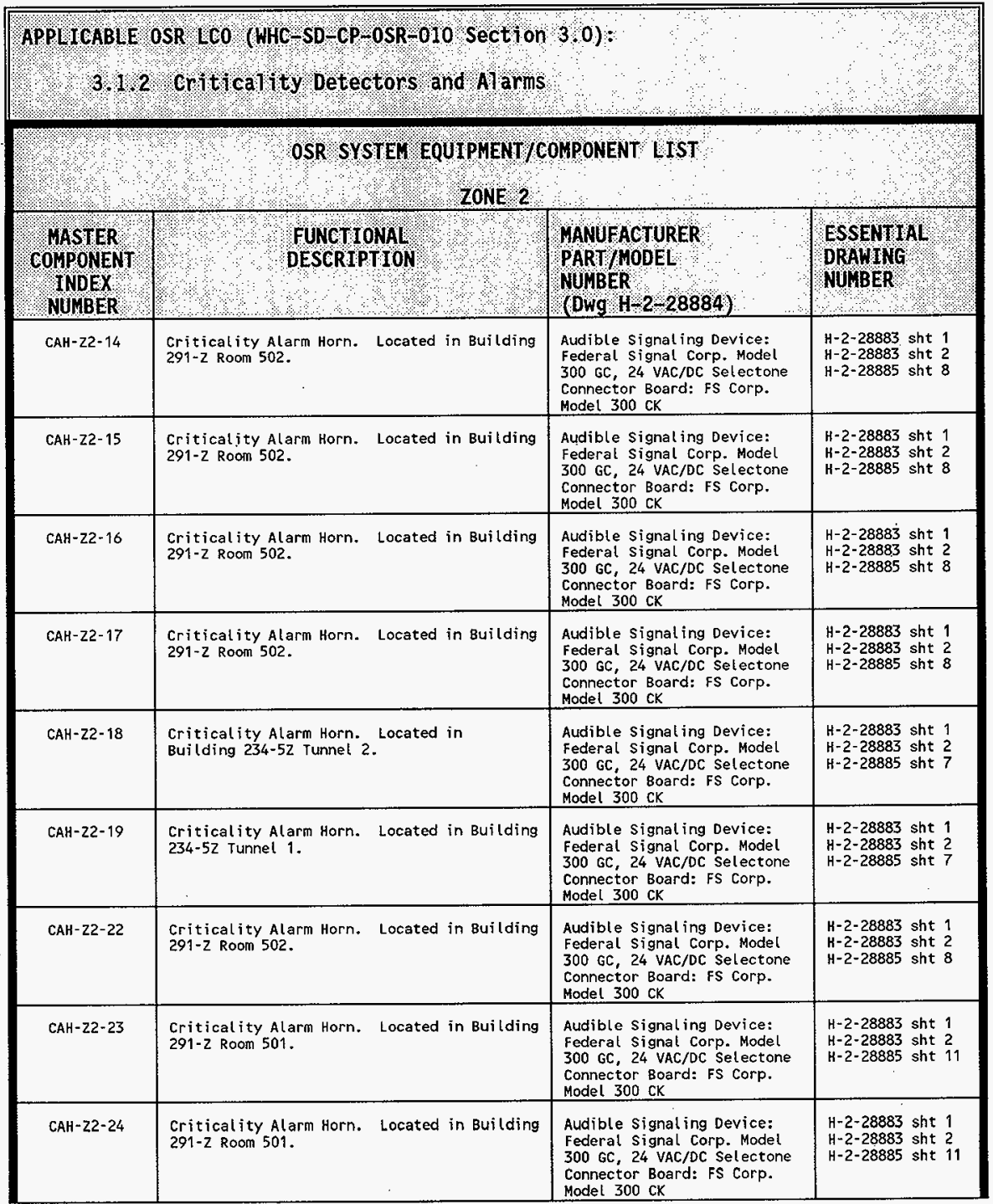




\begin{tabular}{|c|c|c|c|}
\hline \multirow[t]{2}{*}{ 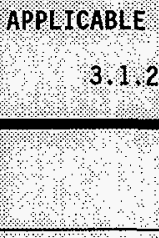 } & 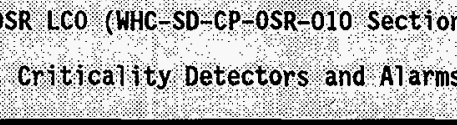 & $\begin{array}{l}30) \div \quad \\
3\end{array}$ & 3 \\
\hline & $\begin{array}{l}\text { OSR SYSTEN EQUIPHENT /C } \\
\text { LONE } 2\end{array}$ & JMPONENT LIST & \\
\hline $\begin{array}{l}\text { MASTER } \\
\text { COMPONENT } \\
\text { NWNEX } \\
\text { NUMBER }\end{array}$ & 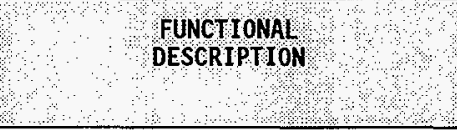 & $\begin{array}{l}\text { MANUFACTURER } \\
\text { PARTYHODEL } \\
\text { NUWBER } \\
\text { (DWg H-2-28884) }\end{array}$ & $\begin{array}{l}\text { ESSENHAL } \\
\text { DRAWHW } \\
\text { NUHBER }\end{array}$ \\
\hline $\mathrm{CAH}-\mathrm{Z2}-25$ & $\begin{array}{l}\text { Criticality Alarm Horn. Located in Building } \\
291-2 \text { Room } 500 \text {. }\end{array}$ & $\begin{array}{l}\text { Audible signal ing Device: } \\
\text { Federal Signal Corp. Model } \\
300 \text { GC, } 24 \text { VAC/DC Selectone } \\
\text { Connector Board: FS Corp. } \\
\text { Model } 300 \text { CK }\end{array}$ & $\begin{array}{l}H-2-28883 \text { sht } 1 \\
H-2-28883 \text { sht } 2 \\
H-2-28885 \text { sht } 11\end{array}$ \\
\hline CALM-Z2-1 & $\begin{array}{l}\text { Criticality Alarm Logic Module. Provides } \\
\text { the logic circuitry for annunciation of } \\
\text { alert and alarm conditions as detected by } \\
\text { the CADMs. Located in CAP-z2. }\end{array}$ & Per Drawing $H-2-91179$ & $\begin{array}{l}H-2-28883 \text { sht } 1 \\
H-2-28883 \text { sht } 2 \\
H-2-28885 \text { sht } 8\end{array}$ \\
\hline CALM-Z2-2 & $\begin{array}{l}\text { Criticality Alarm Logic Module. Provides } \\
\text { the logic circuitry for annunciation of } \\
\text { alert and alarm conditions as detected by } \\
\text { the CADMs. Located in CAP-Z2. }\end{array}$ & Per Drawing $\mathrm{H}-2-91179$ & $\begin{array}{l}H-2-28883 \text { sht } 1 \\
H-2-28883 \text { sht } 2 \\
H-2-28885 \text { sht } 8\end{array}$ \\
\hline $\mathrm{CAP}-\mathrm{Z2}$ & $\begin{array}{l}\text { Criticality Alarm Panel Zone } 2 \text {. Contains } \\
\text { the CADMs, CALMs, CARM, Inhibit switches, } \\
\text { local (building) annunciation and Intertie } \\
\text { relays for the Zone } 2 \text { Criticality Alarm } \\
\text { system. Located in Building } 234-52 \text { Corridor } \\
2 \text {. }\end{array}$ & $\begin{array}{l}\text { Relay Rack. } \\
\text { Premier, TVA } 7719-23\end{array}$ & $\begin{array}{l}\text { H-2-28883 sht } 1 \\
H-2-28883 \text { sht } 2 \\
H-2-28885 \text { sht } 8\end{array}$ \\
\hline CARM-Z2 & $\begin{array}{l}\text { Criticality Alarm Relay Module. Activates } \\
\text { the alarm signal generator and provides an } \\
\text { annunciation signal in the event of a high } \\
\text { alarm signal from the CALM. Includes an } \\
\text { inhibit switch to disable the alarm output } \\
\text { signal. Lacated in CAP-Z2. }\end{array}$ & Per Drawing $\mathrm{H}-2-91180$ & $\begin{array}{l}H-2-28883 \text { sht } 1 \\
H-2-28883 \text { sht } 2 \\
H-2-28885 \text { sht } 8\end{array}$ \\
\hline CBS-Z2-1 & $\begin{array}{l}\text { Central 8attery system. Nominal } 24 \text { VDC } \\
\text { system. Includes one } 12 \text { cell battery } \\
\text { capable of supplying power to the } \\
\text { critical ity alarm system in excess of } 8 \\
\text { hours and charger capable of maintaining the } \\
\text { charge on the battery and while providing } \\
\text { normal power. Primary supply power failure } \\
\text { annunciation. Located adjacent to CAP-z2. }\end{array}$ & $\begin{array}{l}\text { Battery: } \\
\text { Exide Power systems } 12 \text { or } \\
3 \text { CC-5 } \\
24 \text { VDC, } 100 \mathrm{~A} . \\
\text { Charger: } \\
\text { Exide Power systems SCRF } \\
24-1-40-E \\
24 \text { VDC, } 40 \mathrm{~A}\end{array}$ & $\begin{array}{l}H-2-28883 \text { sht } 1 \\
H-2-28883 \text { sht } 2 \\
H-2-28885 \text { sht } 8\end{array}$ \\
\hline $\begin{array}{l}\text { AC POWER } \\
\text { RELAY } \\
\text { (Not required } \\
\text { for labeling } \\
\text { at this } \\
\text { time.) }\end{array}$ & $\begin{array}{l}\text { (SC-3 SE) AC Power Honitor Relay. } \\
\text { Deenergizes on loss of power. A manual } \\
\text { reset is required to prevent a fluctuating } \\
\text { (erratic) power supply from initiating false } \\
\text { alarms. }\end{array}$ & Allen Bradley \#700-C201A1 & $\begin{array}{l}H-2-28883 \text { sht } 1 \\
H-2-28883 \text { sht } 2 \\
H-2-28885 \text { sht } 8\end{array}$ \\
\hline$S-2-1 A$ & $\begin{array}{l}\text { Inhibit Switch for CAD/CADM-Z2-1A used to } \\
\text { prevent annunciation of alarm during test, } \\
\text { cal ibration, and replacement of CADS and } \\
\text { CADMs. Located on CAP-Z2. }\end{array}$ & $\begin{array}{l}\text { Square D Class } 9001 \text {, } \\
\text { Type LRSA-2K1 with } \\
\text { Type LA-1 Contact Blocks }\end{array}$ & $\begin{array}{l}\text { H-2-28883 sht } 1 \\
H-2-28883 \text { sht } 2 \\
H-2-28885 \text { sht } 8\end{array}$ \\
\hline
\end{tabular}




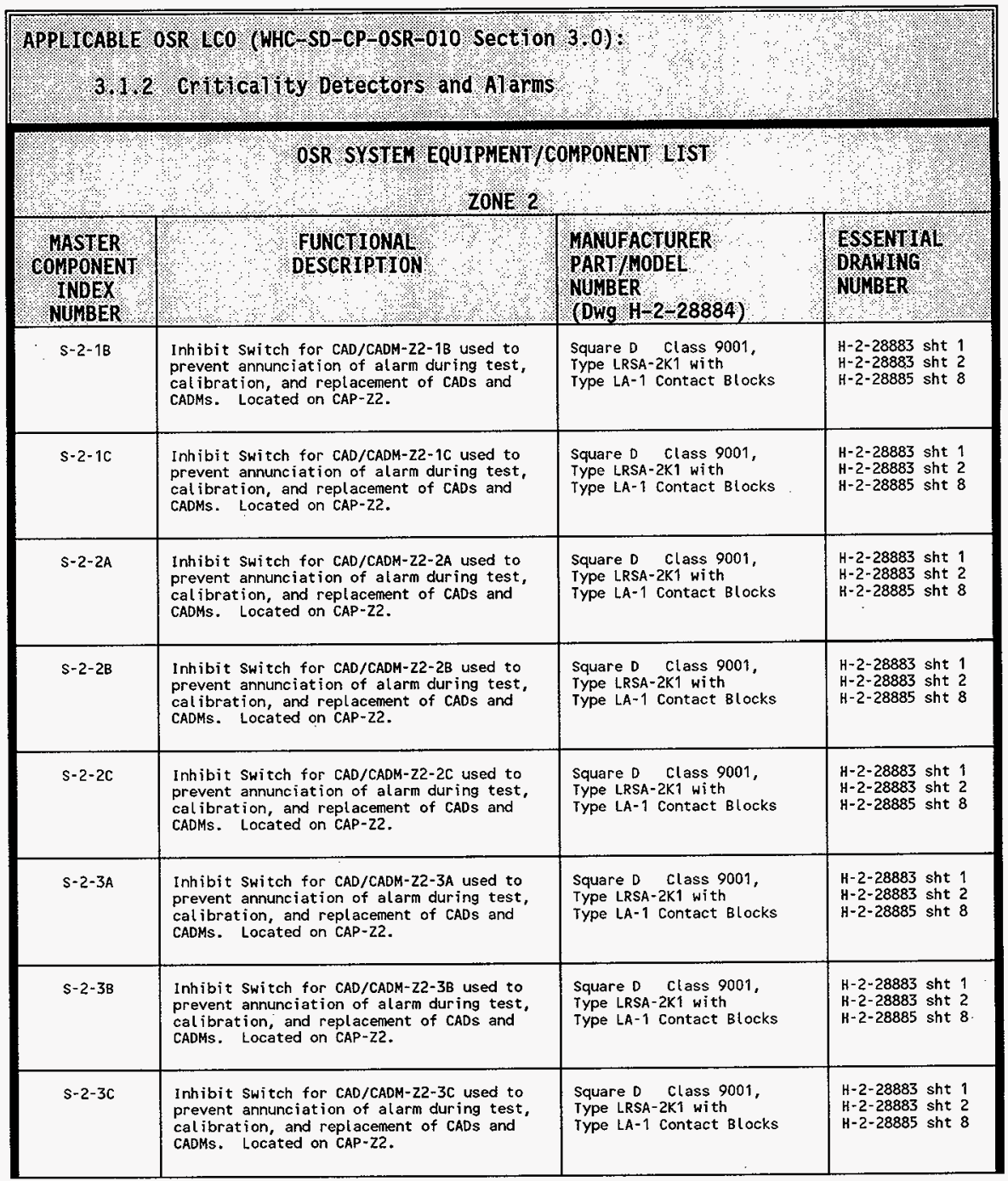




\begin{tabular}{|c|c|c|c|}
\hline \multicolumn{4}{|c|}{ 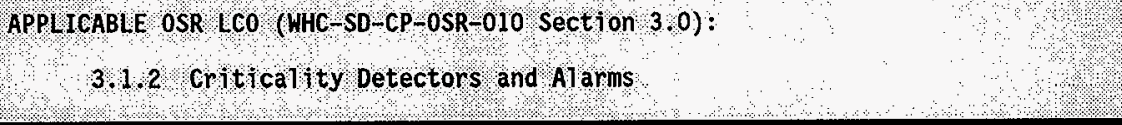 } \\
\hline ? & $\begin{array}{l}\text { OSR SYSTEN EQUTPMENT/ } \\
\text { ZONE? }\end{array}$ & MPONENT LIST & \\
\hline $\begin{array}{l}\text { MASTER } \\
\text { COMPONENT } \\
\text { THDEX } \\
\text { NUNBER }\end{array}$ & $\begin{array}{l}\text { FUNCTIONAL } \\
\text { DESCRIPTION }\end{array}$ & $\begin{array}{l}\text { GANUFACTURER } \\
\text { PART/WODEL } \\
\text { NUMBER } \\
\text { (DWg } 1-2-28884)\end{array}$ & $\begin{array}{l}\text { ESSENTIAL } \\
\text { DRAWING } \\
\text { NUWBER }\end{array}$ \\
\hline$S-2-4 A$ & $\begin{array}{l}\text { Inhibit Switch for CAD/CADM-Z2-4A used to } \\
\text { prevent annunciation of alarm during test, } \\
\text { cal ibration, and replacement of CADs and } \\
\text { CADMs. Located on CAP-Z2. }\end{array}$ & $\begin{array}{l}\text { Square D Class } 9001 \text {, } \\
\text { Type LRSA-2K1 with } \\
\text { Type LA-1 Contact Blocks }\end{array}$ & $\begin{array}{l}H-2-28883 \text { sht } 1 \\
H-2-28883 \text { sht } 2 \\
H-2-28885 \text { sht } 8\end{array}$ \\
\hline$s-2-4 B$ & $\begin{array}{l}\text { Inhibit Switch for CAD/CADM-Z2-4B used to } \\
\text { prevent annunciation of alarm during test, } \\
\text { calibration, and replacement of CADS and } \\
\text { CADMs. Located on CAP-Z2. }\end{array}$ & $\begin{array}{l}\text { Square } D \text { Class } 9001 \text {, } \\
\text { Type LRSA-2K1 with } \\
\text { Type LA-1 Contact Blocks }\end{array}$ & $\begin{array}{l}H-2-28883 \text { sht } 1 \\
H-2-28883 \text { sht } 2 \\
H-2-28885 \text { sht } 8\end{array}$ \\
\hline$s-2-4 c$ & $\begin{array}{l}\text { Inhibit Switch for CAD/CADM-Z2-4C used to } \\
\text { prevent annunciation of a arm during test, } \\
\text { calibration, and replacement of CADS and } \\
\text { CADMs. Located on CAP-Z2. }\end{array}$ & $\begin{array}{l}\text { Square D Class } 9001 \text {, } \\
\text { Type LRSA-2K1 with } \\
\text { Type LA-1 Contact Blocks }\end{array}$ & $\begin{array}{l}H-2-28883 \text { sht } 1 \\
H-2-28883 \text { sht } 2 \\
H-2-28885 \text { sht } 8\end{array}$ \\
\hline
\end{tabular}




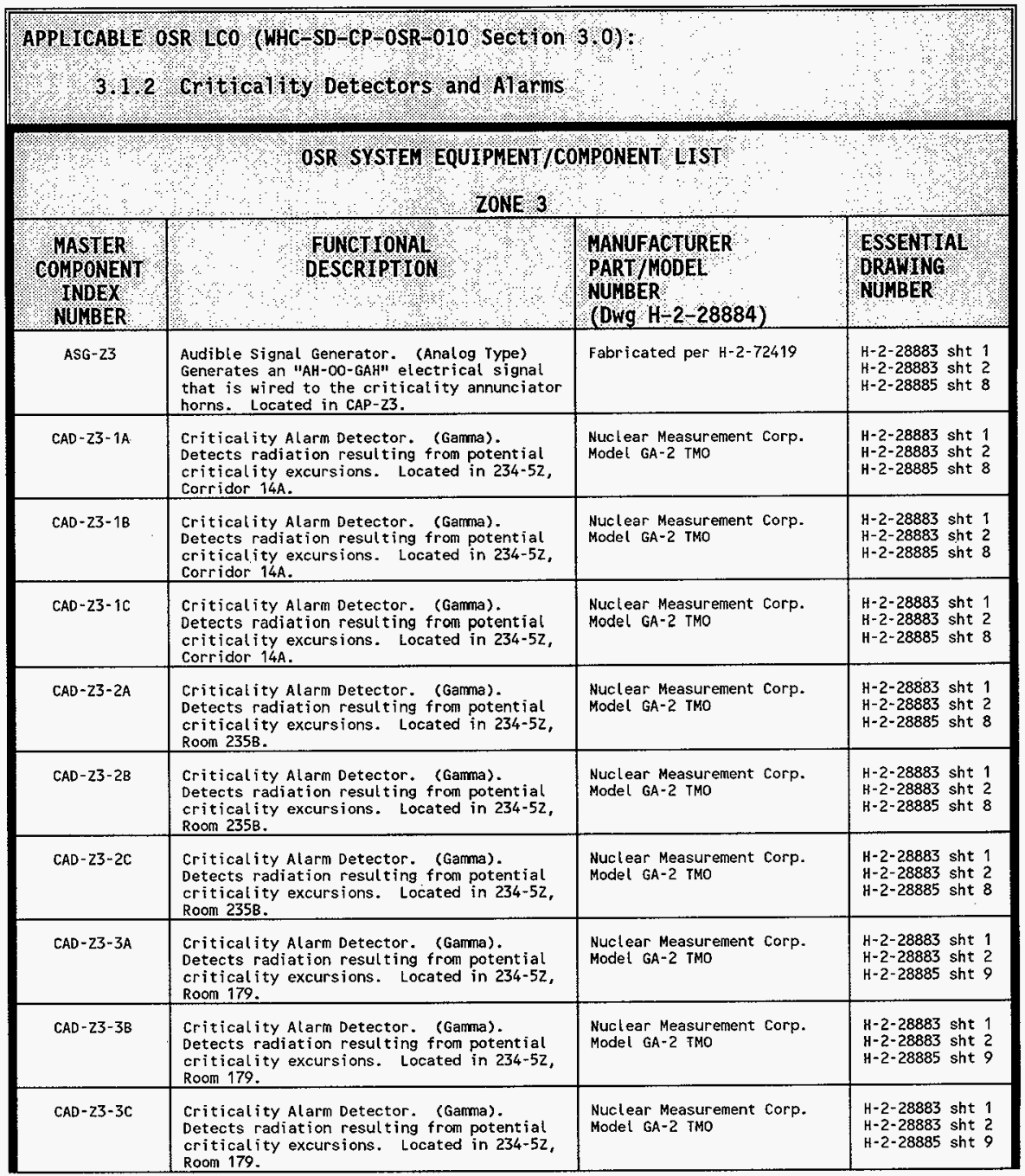




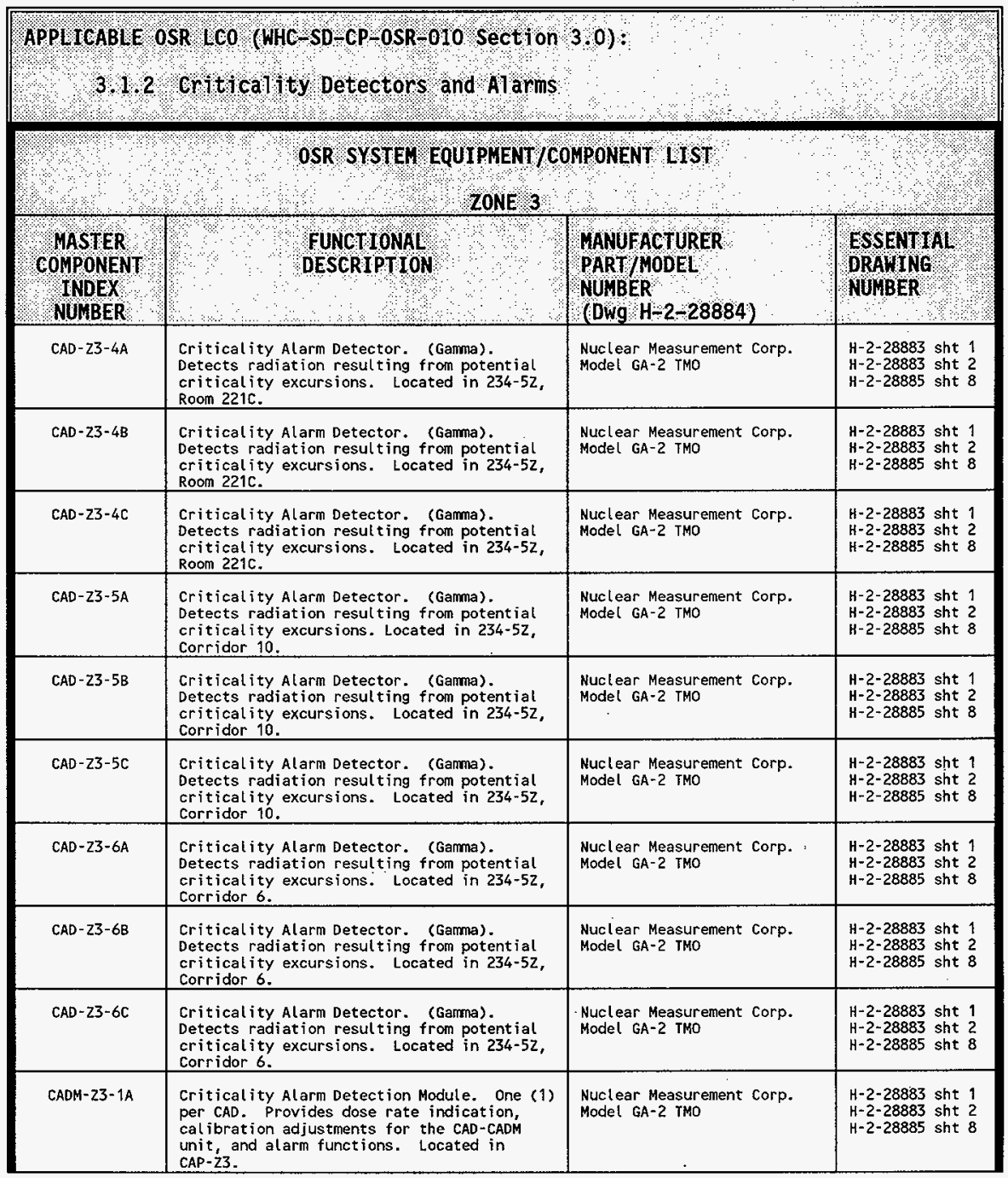




\begin{tabular}{|c|c|c|c|}
\hline \multicolumn{4}{|c|}{ 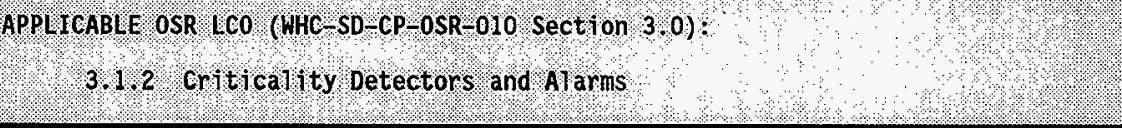 } \\
\hline 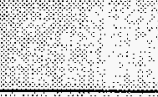 & $\begin{array}{l}\text { OSR SYSTEM EQUIPHENT/ } \\
3 \\
\text { 20NE } 3\end{array}$ & PONENT LIST & \\
\hline $\begin{array}{l}\text { PASTER } \\
\text { GOMPONENT } \\
\text { NHWEX } \\
\text { NOWBER }\end{array}$ & 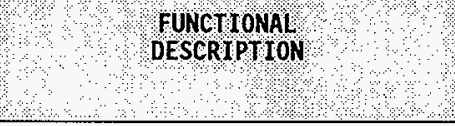 & $\begin{array}{l}\text { WANUFACTURER } \\
\text { PART/MODEL } \\
\text { NUMBER } \\
\text { (DWG H-2-28884) }\end{array}$ & $\begin{array}{l}\text { SSEMHA } \\
\text { ORAWTHG }\end{array}$ \\
\hline CADM $-23-1 C$ & $\begin{array}{l}\text { Criticality Alarm Detection Module. One ( } q) \\
\text { per CAD. Provides dose rate indication, } \\
\text { calibration adjustments for the CAD-CADM } \\
\text { unit, and alarm functions. Located in } \\
\text { CAP-Z3. }\end{array}$ & $\begin{array}{l}\text { Nuclear Measurement Corp. } \\
\text { Model GA-2 TMO }\end{array}$ & $\begin{array}{l}H-2-28883 \text { sht } 1 \\
H-2-28883 \text { sht } 2 \\
H-2-28885 \text { sht } 8\end{array}$ \\
\hline CADM-Z3-2A & $\begin{array}{l}\text { Criticality Alarm Detection Module. One (1) } \\
\text { per CAD. Provides dose rate indication, } \\
\text { cal ibration adjustments for the CAD-CADM } \\
\text { unit, and alarm functions. Located in } \\
\text { CAP-Z3. }\end{array}$ & $\begin{array}{l}\text { Nuclear Measurement Corp. } \\
\text { Model GA-2 THO }\end{array}$ & $\begin{array}{l}H-2-28883 \text { sht } 1 \\
H-2-28883 \text { sht } 2 \\
H-2-28885 \text { sht } 8\end{array}$ \\
\hline CADM-23-2B & $\begin{array}{l}\text { Criticality Alarm Detection Module. One (1) } \\
\text { per CAD. Provides dose rate indication, } \\
\text { cal ibration adjustments for the CAD-CADM } \\
\text { unit, and alarm functions. Located in } \\
\text { CAP-Z3. }\end{array}$ & $\begin{array}{l}\text { Nucl ear Measurement Corp. } \\
\text { Model GA-2 IMO }\end{array}$ & $\begin{array}{l}H-2-28883 \text { sht } 1 \\
H-2-28883 \text { sht } 2 \\
H-2-28885 \text { sht } 8\end{array}$ \\
\hline CADM-23-3A & $\begin{array}{l}\text { Criticality Alarm Detection Module. One (1) } \\
\text { per CAD. Provides dose rate indication, } \\
\text { cal ibration adjustments for the CAD-CADM } \\
\text { unit, and alarm functions. Located in } \\
\text { CAP-Z3. }\end{array}$ & $\begin{array}{l}\text { Nucl ear Measurement Corp. } \\
\text { Model GA-2 TMO }\end{array}$ & $\begin{array}{l}H-2-28883 \text { sht } 1 \\
H-2-28883 \text { sht } 2 \\
H-2-28885 \text { sht } 8\end{array}$ \\
\hline$C A D M-Z 3-3 B$ & $\begin{array}{l}\text { Criticality Alarm Detection Module. One (1) } \\
\text { per CAD. Provides dose rate indication, } \\
\text { cal ibration adjustments for the CAD-CADM } \\
\text { Unit, and alarm functions. Located in } \\
\text { CAP- } Z 3 \text {. }\end{array}$ & $\begin{array}{l}\text { Nuclear Measurement Corp. } \\
\text { Model GA-2 TMO }\end{array}$ & $\begin{array}{l}H-2-28883 \text { sht } 1 \\
H-2-28883 \text { sht } 2 \\
H-2-28885 \text { sht } 8\end{array}$ \\
\hline CADM-Z3-3C & $\begin{array}{l}\text { Criticality Alarm Detection Module. One (1) } \\
\text { per CAD. Provides dose rate indication, } \\
\text { calibration adjustments for the CAD-CADM } \\
\text { unit, and alarm functions. Located in } \\
\text { CAP-Z3. }\end{array}$ & $\begin{array}{l}\text { Nuclear Measurement Corp. } \\
\text { Model GA-2 TMO }\end{array}$ & $\begin{array}{l}H-2-28883 \text { sht } 1 \\
H-2-28883 \text { sht } 2 \\
H-2-28885 \text { sht } 8\end{array}$ \\
\hline CADM-Z3-4A & $\begin{array}{l}\text { Criticality Alarm Detection Module. One (1) } \\
\text { per CAD. Provides dose rate indication, } \\
\text { calibration adjustments for the CAD-CADM } \\
\text { unit, and alarm functions. Located in } \\
\text { CAP- } 23 \text {. }\end{array}$ & $\begin{array}{l}\text { Nucl ear Measurement Corp. } \\
\text { Model GA-2 rMO }\end{array}$ & $\begin{array}{l}H-2-28883 \text { sht } ? \\
H-2-28883 \text { sht } 2 \\
H-2-28885 \text { sht } 8\end{array}$ \\
\hline
\end{tabular}




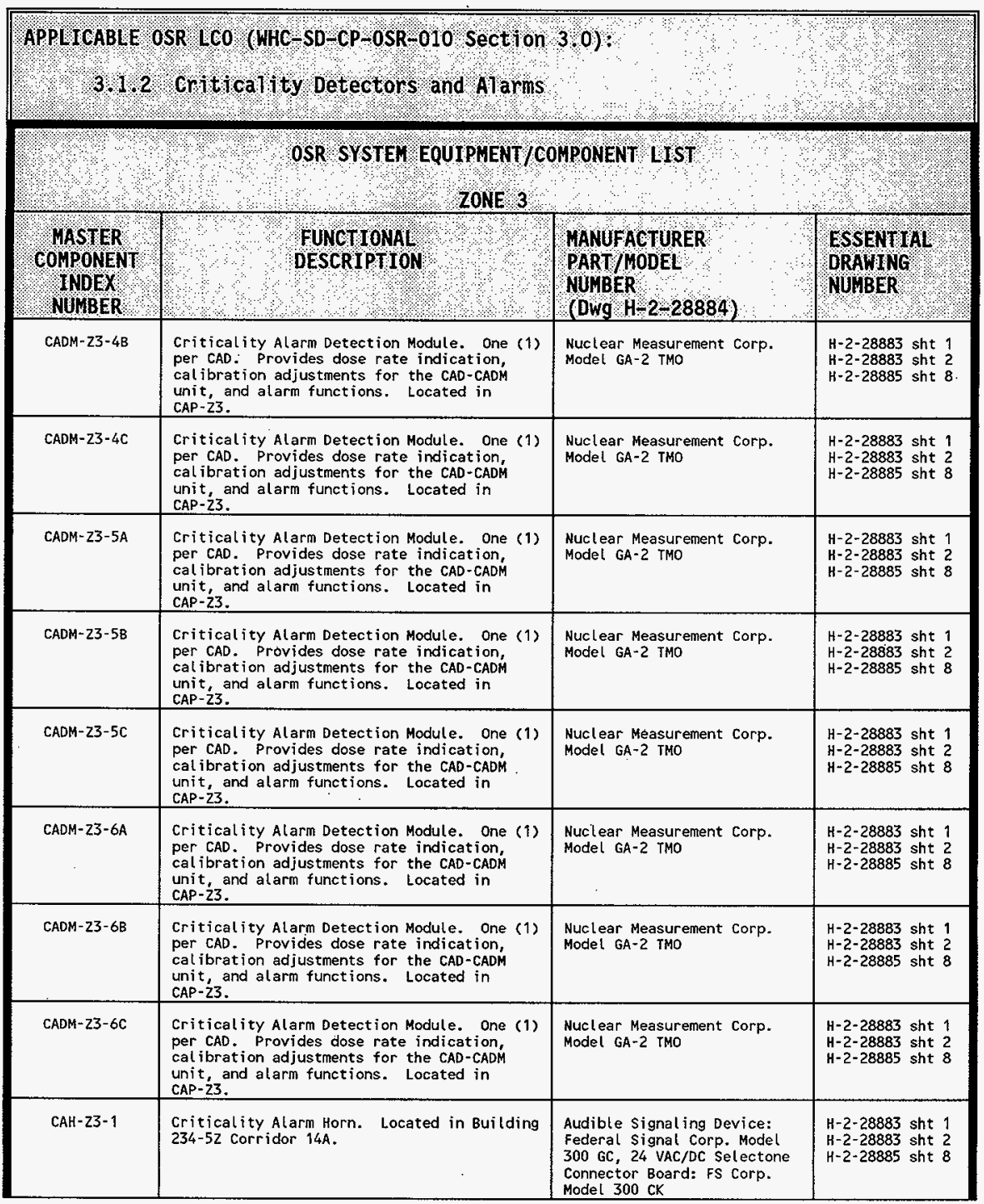




\begin{tabular}{|c|c|c|c|}
\hline \multicolumn{4}{|c|}{ 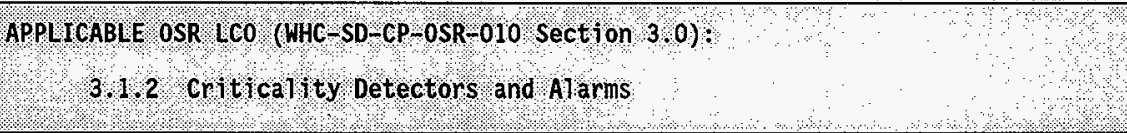 } \\
\hline अ & 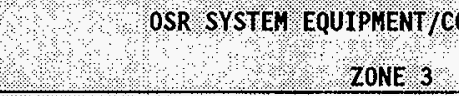 & PONENT LTST & \\
\hline $\begin{array}{l}\text { MASTER } \\
\text { COMPONENT } \\
\text { NONDEX } \\
\text { NUMBER }\end{array}$ & 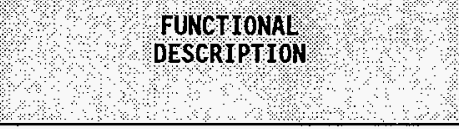 & $\begin{array}{l}\text { WANOFACTURER } \\
\text { PART/MODEL } \\
\text { NUMBER } \\
\text { (DWg }\end{array}$ & 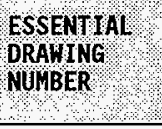 \\
\hline CAH-Z3-3 & $\begin{array}{l}\text { Criticality Alarm Horn. Located in Building } \\
234-52 \text { Room 2358. }\end{array}$ & $\begin{array}{l}\text { Audible Signaling Device: } \\
\text { Federal signal Corp. Model } \\
300 \text { GC, } 24 \text { VAC/DC Selectone } \\
\text { Connector Board: FS Corp. } \\
\text { Madel } 300 \text { CK }\end{array}$ & $\begin{array}{l}H-2-28883 \text { sht } 1 \\
H-2-28883 \text { sht } 2 \\
H-2-28885 \text { sht } 8\end{array}$ \\
\hline CAH-Z3-4 & $\begin{array}{l}\text { Criticality Alarm Horn. Located in Building } \\
234-52 \text { Room } 230 c \text {. }\end{array}$ & $\begin{array}{l}\text { Audible Signal ing Device: } \\
\text { Federal Signal Corp. Model } \\
300 \text { GC, } 24 \text { VAC/OC Selectone } \\
\text { Connector Board: FS Corp. } \\
\text { Model } 300 \text { CK }\end{array}$ & $\begin{array}{l}H-2-28883 \text { sht } 1 \\
H-2-28883 \text { sht } 2 \\
H-2-28885 \text { sht } 8\end{array}$ \\
\hline CAH-Z3-5 & $\begin{array}{l}\text { Criticality Alarm Horn. Located in Building } \\
234-5 Z \text { Room } 170 .\end{array}$ & $\begin{array}{l}\text { Audible signaling Device: } \\
\text { Federal Signal Corp. Model } \\
300 \text { GC, } 24 \text { VAC/DC Selectone } \\
\text { Connector Board: FS Corp. } \\
\text { Model } 300 \mathrm{CK}\end{array}$ & $\begin{array}{l}H-2-28883 \text { sht } 1 \\
H-2-28883 \text { sht } 2 \\
H-2-28885 \text { sht } 8\end{array}$ \\
\hline $\mathrm{CAH}-\mathrm{Z3}-7$ & $\begin{array}{l}\text { Criticality Alarm Horn. Located in Building } \\
234-52 \text { Corridor } 6 \text {. }\end{array}$ & $\begin{array}{l}\text { Audible Signaling Device: } \\
\text { Federat Signal Corp. Model } \\
300 \mathrm{GC}, 24 \text { VAC/DC Selectone } \\
\text { Connector Board: FS Corp. } \\
\text { Model } 300 \text { CK }\end{array}$ & $\begin{array}{l}H-2-28883 \text { sht } 1 \\
H-2-28883 \text { sht } 2 \\
H-2-28885 \text { sht } 8\end{array}$ \\
\hline $\mathrm{CAH}-\mathrm{Z3}-8$ & $\begin{array}{l}\text { Criticality Alarm Horn. Located in Building } \\
234-52 \text { Room } 179 \text {. }\end{array}$ & $\begin{array}{l}\text { Audible Signaling Device: } \\
\text { Federal Signal Corp. Model } \\
300 \text { GC, } 24 \text { VAC/DC Selectone } \\
\text { Connector Board: FS Corp. } \\
\text { Model } 300 \text { CK }\end{array}$ & $\begin{array}{l}H-2-28883 \text { sht } 1 \\
H-2-28883 \text { sht } 2 \\
H-2-28885 \text { sht } 8\end{array}$ \\
\hline $\mathrm{CAH}-23-9$ & $\begin{array}{l}\text { Criticality Alarm Horn. Located in Building } \\
2734-\mathrm{ZG} \text {. }\end{array}$ & $\begin{array}{l}\text { Audible Signaling Device: } \\
\text { Federal Signal Corp. Model } \\
300 \text { GC, } 24 \text { VAC/DC Selectone } \\
\text { Connector Board: FS Corp. } \\
\text { Model } 300 \text { CK }\end{array}$ & $\begin{array}{l}H-2-28883 \text { sht } 1 \\
H-2-28883 \text { sht } 2 \\
H-2-28885 \text { sht } 8\end{array}$ \\
\hline $\mathrm{CAH}-23-10$ & $\begin{array}{l}\text { Criticality Alarm Horn. Located in } \\
\text { Building } 234-52 \text { Room } 264 \text {. }\end{array}$ & $\begin{array}{l}\text { Audible signal ing Device: } \\
\text { Federal signal Corp. Model } \\
300 \text { GC, } 24 \text { VAC/DC Selectone } \\
\text { Connector Board: FS Corp. } \\
\text { Model } 300 \text { CK }\end{array}$ & $\begin{array}{l}H-2-28883 \text { sht } 1 \\
H-2-28883 \text { sht } 2 \\
H-2-28885 \text { sht } 9\end{array}$ \\
\hline
\end{tabular}




\begin{tabular}{|c|c|c|c|}
\hline \multirow{2}{*}{\multicolumn{4}{|c|}{ 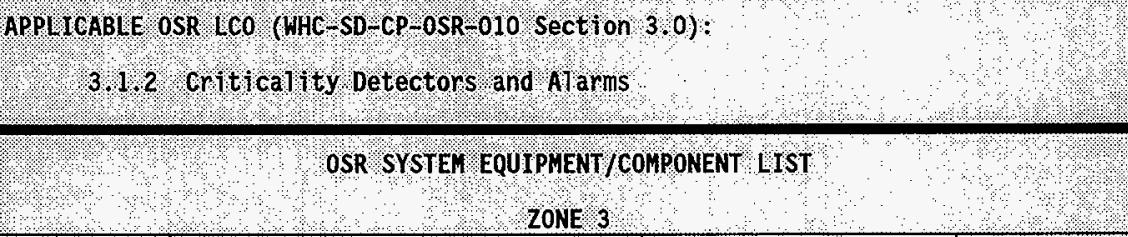 }} \\
\hline & & & \\
\hline $\begin{array}{l}\text { MASTER } \\
\text { CONPONENT } \\
\text { WNDEX } \\
\text { NUMBER }\end{array}$ & 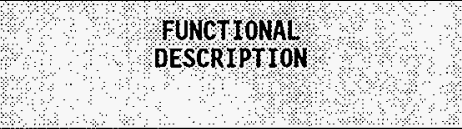 & $\begin{array}{l}\text { WANUPACTURER } \\
\text { PART/HODEL } \\
\text { NUMBER } \\
\text { (DWG } H-2-28884)\end{array}$ & $\begin{array}{l}\text { SSSENHAL } \\
\text { DRAWTNG } \\
\text { NUHBER }\end{array}$ \\
\hline CAH-23-12 & $\begin{array}{l}\text { Criticality Alarm Horn. Located in Building } \\
234-52 \text { Room } 264 \text {. }\end{array}$ & $\begin{array}{l}\text { Audible Signaling Device: } \\
\text { Federal Signal Corp. Model } \\
300 \text { GC, } 24 \text { VAC/OC Selectone } \\
\text { Connector Board: FS Corp. } \\
\text { Model } 300 \text { CK }\end{array}$ & $\begin{array}{l}H-2-28883 \text { sht } 1 \\
H-2-28883 \text { sht } 2 \\
H-2-28885 \text { sht } 9\end{array}$ \\
\hline CAH $-23-13$ & $\begin{array}{l}\text { Criticality Alarm Horn. Located in Building } \\
234-52 \text { Room } 262 \text {. }\end{array}$ & $\begin{array}{l}\text { Audible Signaling Device: } \\
\text { Federal Signal Corp. Model } \\
300 \text { GC, } 24 \text { VAC/DC Selectone } \\
\text { Connector Board: FS Corp. } \\
\text { Model } 300 \text { CK }\end{array}$ & $\begin{array}{l}H-2-28883 \text { sht } 1 \\
H-2-28883 \text { sht } 2 \\
H-2-28885 \text { sht } 9\end{array}$ \\
\hline CAH $-23-14$ & $\begin{array}{l}\text { Criticality Alarm Horn. Located in Building } \\
234-52 \text { Room } 262 \text {. }\end{array}$ & $\begin{array}{l}\text { Audible signaling Device: } \\
\text { Federal Signal Corp. Model } \\
300 \text { GC, } 24 \text { VAC/DC Selectone } \\
\text { Connector Board: FS Corp. } \\
\text { Model } 300 \text { CK }\end{array}$ & $\begin{array}{l}H-2-28883 \text { sht } 1 \\
H-2-28883 \text { sht } 2 \\
H-2-28885 \text { sht } 9\end{array}$ \\
\hline CALM-23-1 & $\begin{array}{l}\text { Criticality Alarm Logic Module. Provides } \\
\text { the logic circuitry for annunciation of } \\
\text { alert and alarm conditions as detected by } \\
\text { the CADMs. Located in CAP- } 23 \text {. }\end{array}$ & Per Drawing $\mathrm{H}-2-91179$ & $\begin{array}{l}H-2-28883 \text { sht } 1 \\
H-2-28883 \text { sht } 2 \\
H-2-28885 \text { sht } 8\end{array}$ \\
\hline CALM-23-2 & $\begin{array}{l}\text { Criticality Alarm Logic Module. Provides } \\
\text { the logic circuitry for annunciation of } \\
\text { alert and alarm conditions as detected by } \\
\text { the CADMs. Located in CAP- } 23 \text {. }\end{array}$ & Per Drawing H-2-91179 & $\begin{array}{l}H-2-28883 \text { sht } 1 \\
H-2-28883 \text { sht } 2 \\
H-2-28885 \text { sht } 8\end{array}$ \\
\hline CALM- 23-3 & $\begin{array}{l}\text { Criticality Alarm Logic Module. Provides } \\
\text { the logic circuitry for annunciation of } \\
\text { atert and alarm conditions as detected by } \\
\text { the CADMs. Located in CAP-23. }\end{array}$ & Per Drawing $\mathrm{H}-2-91179$ & $\begin{array}{l}H-2-28883 \text { sht } 1 \\
H-2-28883 \text { sht } 2 \\
H-2-28885 \text { sht } 8\end{array}$ \\
\hline CALM- $23-4$ & $\begin{array}{l}\text { Criticality Alarm Logic Module. Provides } \\
\text { the logic circuitry for annunciation of } \\
\text { alert and alarm conditions as detected by } \\
\text { the CADMs. Located in CAP- } 23 \text {. }\end{array}$ & Per Drawing $\mathrm{H}-2-91179$ & $\begin{array}{l}H-2-28883 \text { sht } 1 \\
H-2-28883 \text { sht } 2 \\
H-2-28885 \text { sht } 8\end{array}$ \\
\hline
\end{tabular}




\begin{tabular}{|c|c|c|c|}
\hline \multicolumn{4}{|c|}{ 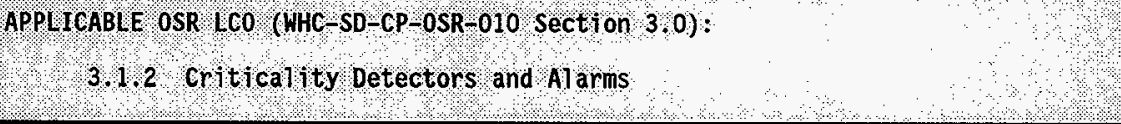 } \\
\hline 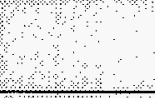 & $\begin{array}{r}\text { OSR SYSTEN EQUTPNENT/C } \\
\text { S } \\
\end{array}$ & MPONENT IIST & \\
\hline $\begin{array}{l}\text { MASTER } \\
\text { COMPONENT } \\
\text { NUWBER }\end{array}$ & PFUNCTIONAL & $\begin{array}{l}\text { WANUFACTURER } \\
\text { PART FODEL } \\
\text { HUNBER } \\
\text { (DWg H-2-28884) }\end{array}$ & 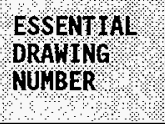 \\
\hline CARM -23 & $\begin{array}{l}\text { Critical ity Alarm Relay Module. Activates } \\
\text { the alarm signal generator and provides an } \\
\text { annunciation signal in the event of a high } \\
\text { alarm signal from the CALM. Includes an } \\
\text { inhibit switch to disable the alarm output } \\
\text { signal. Located in CAP-23. }\end{array}$ & Per Drawing $\mathrm{H}-2-91180$ & $\begin{array}{l}H-2-28883 \text { sht } 1 \\
H-2-28883 \text { sht } 2 \\
H-2-28885 \text { sht } 8\end{array}$ \\
\hline CBS-23-1 & $\begin{array}{l}\text { Central Battery System. Nominal } 24 \text { VOC } \\
\text { system. Includes one } 12 \text { cell battery } \\
\text { capable of supplying power to the } \\
\text { criticality alarm system in excess of } 8 \\
\text { hours and charger capable of maintaining the } \\
\text { charge on the battery and while providing } \\
\text { normal power. Primary supply power failure } \\
\text { annunciation. Located adjacent to CAP-z3. }\end{array}$ & $\begin{array}{l}\text { Battery: } \\
\text { Exide Power Systems } 12 \text { or } \\
3 \text { CC-5 } \\
24 \text { VDC, } 100 \mathrm{~A} . \\
\text { Charger: } \\
\text { Exide Power systems SCRF } \\
24-1-40-E \\
24 \text { VDC, } 25 \mathrm{~A}\end{array}$ & $\begin{array}{l}H-2-28883 \text { sht } 1 \\
H-2-28883 \text { sht } 2 \\
H-2-28885 \text { sht } 8\end{array}$ \\
\hline$s-3-1 A$ & $\begin{array}{l}\text { Inhibit Switch for CAD/CADM- } 23-1 \mathrm{~A} \text { used to } \\
\text { prevent annunciation of alarm during test, } \\
\text { cal ibration, and replacement of CADS and } \\
\text { CADMs. Located on CAP-Z3. }\end{array}$ & $\begin{array}{l}\text { Square D CLass } 9001 \text {, } \\
\text { Type LRSA-2K1 with } \\
\text { Type LA-1 Contact Blocks }\end{array}$ & $\begin{array}{l}H-2-28883 \text { sht } 1 \\
H-2-28883 \text { sht } 2 \\
H-2-28885 \text { sht } 8\end{array}$ \\
\hline$s-3-1 B$ & $\begin{array}{l}\text { Inhibit Switch for CAD/CADM- } 23-18 \text { used to } \\
\text { prevent annunciation of alarm during test, } \\
\text { calibration, and replacement of CADS and } \\
\text { CADMs. Located on CAP-Z3. }\end{array}$ & $\begin{array}{l}\text { Square D Class } 9001, \\
\text { Type LRSA-2K1 with } \\
\text { Type LA-1 Contact Blocks }\end{array}$ & $\begin{array}{l}H-2-28883 \text { sht } 1 \\
H-2-28883 \text { sht } 2 \\
H-2-28885 \text { sht } 8\end{array}$ \\
\hline$s-3-1 c$ & $\begin{array}{l}\text { Inhibit Switch for CAD/CADM- } 23-1 C \text { used to } \\
\text { prevent annunciation of alarm during test, } \\
\text { calibration, and replacement of CADs and } \\
\text { CADMs. Located on CAP-23. }\end{array}$ & $\begin{array}{l}\text { Square D Class } 9001, \\
\text { Type LRSA-2K1 with } \\
\text { Type LA-1 Contact Blocks }\end{array}$ & $\begin{array}{l}H-2-28883 \text { sht } 1 \\
H-2-28883 \text { sht } 2 \\
H-2-28885 \text { sht } 8\end{array}$ \\
\hline
\end{tabular}




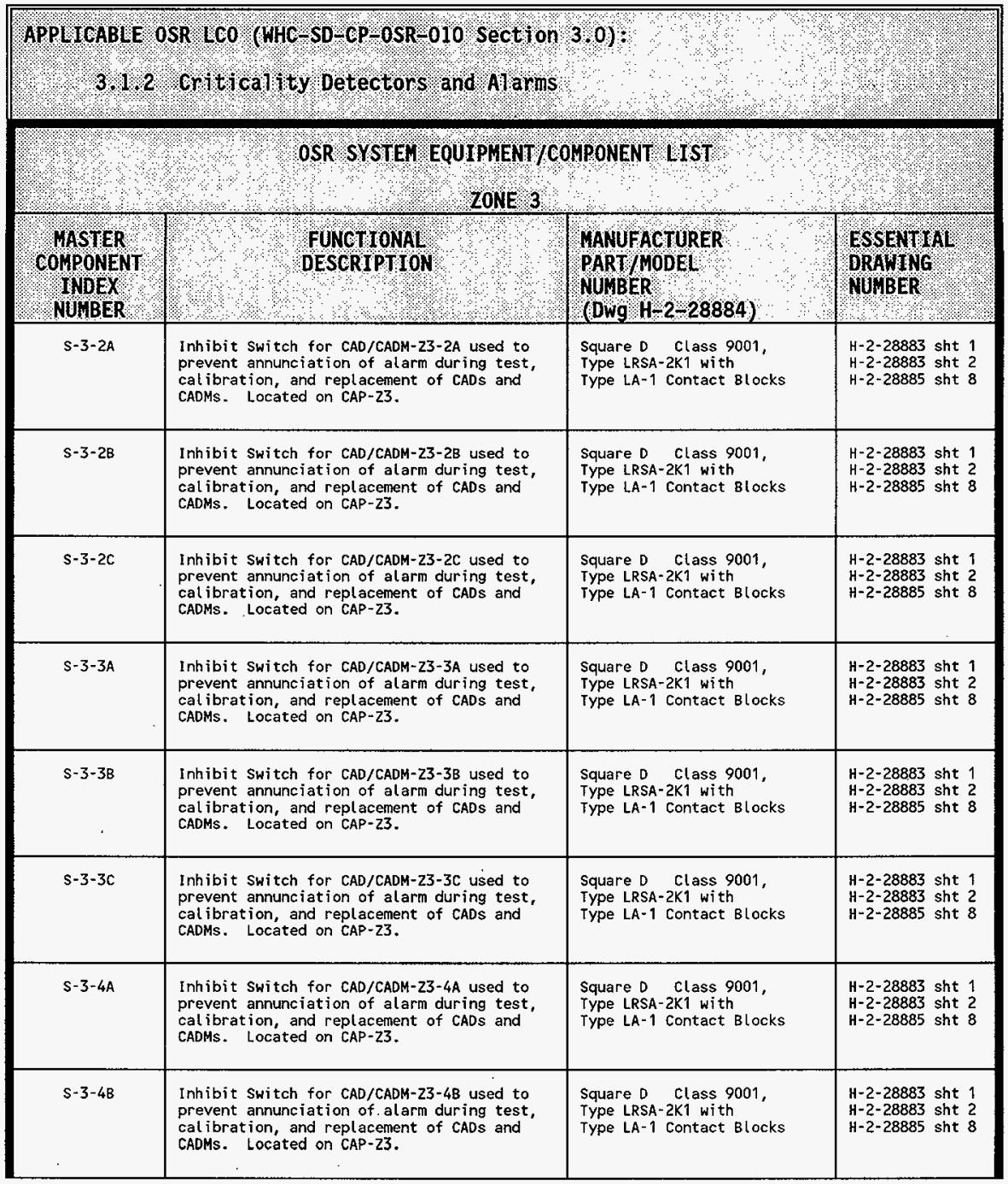




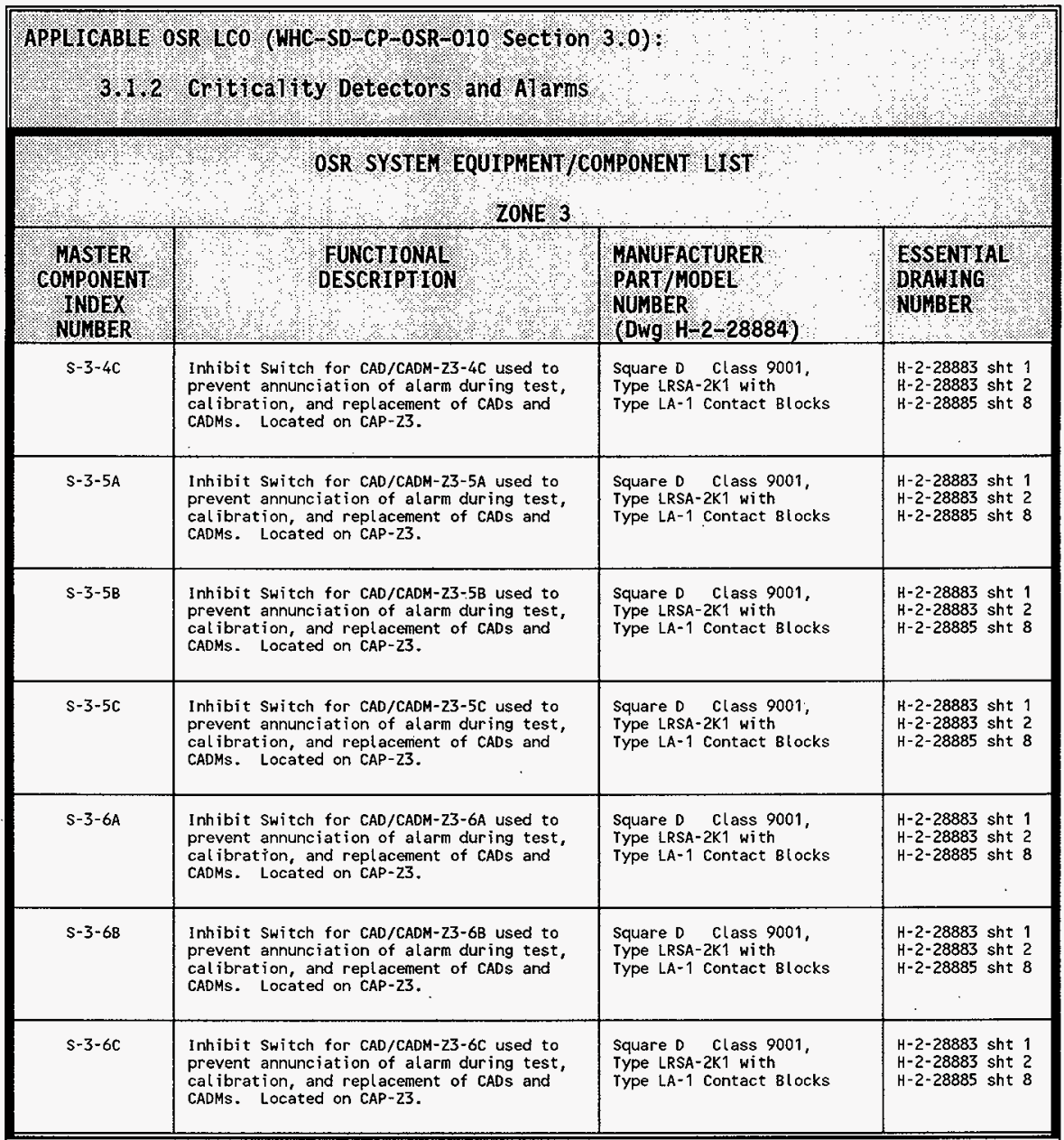

APPLICABLE OSR LCO (WHC SD-CP-0SR-010 section 3.0):

\subsection{Critical ity Detectors and Al arms}




\begin{tabular}{|c|c|c|c|}
\hline 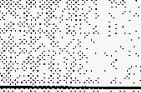 & OOSR SYSTEN EQUTPMENT & MPONENT LIST & \\
\hline $\begin{array}{l}\text { WASTER } \\
\text { OMPONENT } \\
\text { WNDEX } \\
\text { NUMBER }\end{array}$ & PFUNCTIONAL DCRIPT ION, & $\begin{array}{l}\text { WANUFACTURER } \\
\text { PART/MODEL } \\
\text { NUNBER } \\
\text { (DWG }-2-28884 \text { ) }\end{array}$ & 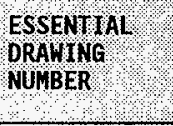 \\
\hline$A S G-24$ & $\begin{array}{l}\text { Audible signal Generator. (Analog Type) } \\
\text { Generates an "AH-OO-GAH" electrical signal } \\
\text { that is wired to the criticality annunciator } \\
\text { horns. Located in CAP-24. }\end{array}$ & Fabricated per H-2-72419 & $\begin{array}{l}H-2-28883 \text { sht } 1 \\
H-2-28883 \text { sht } 2 \\
H-2-28885 \text { sht } 8\end{array}$ \\
\hline$C A D-24-1 A$ & $\begin{array}{l}\text { Criticality Alarm Detector. (Gamma). } \\
\text { Detects radiation resulting from potential } \\
\text { criticality excursions. Located in } 234-52 \text {, } \\
\text { Room } 236 \text {. }\end{array}$ & $\begin{array}{l}\text { Nuclear Measurement Corp. } \\
\text { Model GA-2 THO }\end{array}$ & $\begin{array}{l}H-2-28883 \text { sht } 1 \\
H-2-28883 \text { sht } 2 \\
H-2-28885 \text { sht } 8\end{array}$ \\
\hline$C A D-24-1 B$ & $\begin{array}{l}\text { Criticality Alarm Detector. (Gamma). } \\
\text { Detects radiation resulting from potential } \\
\text { criticality excursions. Located in } 234-52 \text {, } \\
\text { Room } 236 \text {. }\end{array}$ & $\begin{array}{l}\text { Nuclear Measurement Corp. } \\
\text { Model GA-2 TMO }\end{array}$ & $\begin{array}{l}H-2-28883 \text { sht } 1 \\
H-2-28883 \text { sht } 2 \\
H-2-28885 \text { sht } 8\end{array}$ \\
\hline$C A D-24-1 C$ & $\begin{array}{l}\text { Critical ity Alarm Detector. (Gamma). } \\
\text { Detects radiation resulting from potential } \\
\text { critical ity excursions. Located in } 234-52 \text {, } \\
\text { Room } 236 \text {. }\end{array}$ & $\begin{array}{l}\text { Nuclear Measurement Corp. } \\
\text { Hodel GA-2 TMO }\end{array}$ & $\begin{array}{l}H-2-28883 \text { sht } 1 \\
H-2-28883 \text { sht } 2 \\
H-2-28885 \text { sht } 8\end{array}$ \\
\hline CAD $-24-2 A$ & $\begin{array}{l}\text { Critical ity Alarm Detector. (Gamma). } \\
\text { Detects radiation resulting from potential } \\
\text { critical ity excursions. Located in } 234-52 \text {, } \\
\text { Corricor } 239 \mathrm{~A} \text {. }\end{array}$ & $\begin{array}{l}\text { Nuclear Measurement Corp. } \\
\text { Model GA-2 TMO }\end{array}$ & $\begin{array}{l}H-2-28883 \text { sht } 1 \\
H-2-28883 \text { sht } 2 \\
H-2-28885 \text { sht } 8\end{array}$ \\
\hline$C A D-Z 4-2 B$ & $\begin{array}{l}\text { Critical ity Alarm Detector. (Gamma). } \\
\text { Detects radiation resulting from potential } \\
\text { criticality excursions. Located in } 234-52 \text {, } \\
\text { Corridor } 239 \mathrm{~A} \text {. }\end{array}$ & $\begin{array}{l}\text { Nuclear Measurement Corp. } \\
\text { Hodel GA-2 IMO }\end{array}$ & $\begin{array}{l}H-2-28883 \text { sht } 1 \\
H-2-28883 \text { sht } 2 \\
H-2-28885 \text { sht } 8\end{array}$ \\
\hline CAD-Z4-2C & $\begin{array}{l}\text { Criticality Alarm Detector. (Gama). } \\
\text { Detects radiation resulting from potential } \\
\text { critical ity excursions. Located in } 234-52 \text {, } \\
\text { Corridor } 239 \mathrm{~A} \text {. }\end{array}$ & $\begin{array}{l}\text { Nuclear Measurement Corp. } \\
\text { Model GA-2 TMO }\end{array}$ & $\begin{array}{l}H-2-28883 \text { sht } 1 \\
H-2-28883 \text { sht } 2 \\
H-2-28885 \text { sht } 8\end{array}$ \\
\hline$C A D-24-3 A$ & $\begin{array}{l}\text { Criticality Alarm Detector. (Gamma). } \\
\text { Detects radiation resulting from potential } \\
\text { criticality excursions. Located in } 234-52 \text {, } \\
\text { Room } 264 \text {. }\end{array}$ & $\begin{array}{l}\text { Nuclear Measurement Corp. } \\
\text { Model GA-2 TMO }\end{array}$ & $\begin{array}{l}H-2-28883 \text { sht } 1 \\
H-2-28883 \text { sht } 2 \\
H-2-28885 \text { sht } 9\end{array}$ \\
\hline$C A D-24-3 B$ & $\begin{array}{l}\text { Criticality Alarm Detector. (Gamma). } \\
\text { Detects radiation resulting from potential } \\
\text { critical ity excursions. Located in } 234-52 \text {, } \\
\text { Room } 264 \text {. }\end{array}$ & $\begin{array}{l}\text { Nuclear Measurement Corp. } \\
\text { Model GA-2 TMO }\end{array}$ & $\begin{array}{l}H-2-28883 \text { sht } 1 \\
H-2-28883 \text { sht } 2 \\
H-2-28885 \text { sht } 9\end{array}$ \\
\hline$C A D-24-3 C$ & $\begin{array}{l}\text { Criticality Alarm Detector. (Gamma). } \\
\text { Detects radiation resulting from potential } \\
\text { critical ity excursions. Located in } 234-52 \text {, } \\
\text { Room } 264 \text {. }\end{array}$ & $\begin{array}{l}\text { Nucl ear Measurement Corp. } \\
\text { Model GA-2 TMO }\end{array}$ & $\begin{array}{l}H-2-28883 \text { sht } 1 \\
H-2-28883 \text { sht } 2 \\
H-2-28885 \text { sht } 9\end{array}$ \\
\hline$C A D-24=4 A$ & $\begin{array}{l}\text { Criticality Alarm Detector. (Gamma). } \\
\text { Detects radiation resulting from potential } \\
\text { critical ity excursions. Located in } 234-52 \text {, } \\
\text { Corridor } 3 \text {. }\end{array}$ & $\begin{array}{l}\text { Nuclear Measurement Corp. } \\
\text { Model GA-2 TMO }\end{array}$ & $\begin{array}{l}H-2-28883 \text { sht } 1 \\
H-2-28883 \text { sht } 2 \\
H-2-28885 \text { sht } 10\end{array}$ \\
\hline$C A D-24-4 B$ & $\begin{array}{l}\text { Criticality Alarm Detector. (Gamma). } \\
\text { Detects radiation resulting from potential } \\
\text { criticality excursions. Located in } 234-52 \text {, } \\
\text { Corridor } 3 \text {. }\end{array}$ & $\begin{array}{l}\text { Nuclear Measurement Corp. } \\
\text { Model GA-2 TMO }\end{array}$ & $\begin{array}{l}H-2-28883 \text { sht } 1 \\
H-2-28883 \text { sht } 2 \\
H-2-28885 \text { sht } 10\end{array}$ \\
\hline
\end{tabular}




\begin{tabular}{|c|c|c|c|}
\hline$C A D-Z 4-4 C$ & $\begin{array}{l}\text { Critical ity Alarm Detector. (Gamma). } \\
\text { Detects radiation resulting from potential } \\
\text { criticality excursions. Located in } 234-52 \text {, } \\
\text { Corridor } 3 \text {. }\end{array}$ & $\begin{array}{l}\text { Nuclear Measurement Corp. } \\
\text { Model GA-2 TMO }\end{array}$ & $\begin{array}{l}H-2-28883 \text { sht } 1 \\
H-2-28883 \text { sht } 2 \\
H-2-28885 \text { sht } 10\end{array}$ \\
\hline$C A D-Z 4-5 A$ & $\begin{array}{l}\text { Criticality Alarm Detector. (Gama). } \\
\text { Detects radiation resulting from potential } \\
\text { critical ity excursions. Located in } 234-52 \text {, } \\
\text { Room } 1928 \text {. }\end{array}$ & $\begin{array}{l}\text { Nuclear Measurement Corp. } \\
\text { Model GA-2 TMO }\end{array}$ & $\begin{array}{l}H-2-28883 \text { sht } 1 \\
H-2-28883 \text { sht } 2 \\
H-2-28885 \text { sht } 8\end{array}$ \\
\hline$C A D-Z 4-5 B$ & $\begin{array}{l}\text { Criticality Alarm Detector. (Gamma), } \\
\text { Detects radiation resulting from potential } \\
\text { criticality excursions. Located in } 234-52 \text {, } \\
\text { Room } 192 \mathrm{~B} \text {. }\end{array}$ & $\begin{array}{l}\text { Nuctear Measurement Corp. } \\
\text { Model GA-2 IMO }\end{array}$ & $\begin{array}{l}H-2-28883 \text { sht } 1 \\
H-2-28883 \text { sht } 2 \\
H-2-28885 \text { sht } 8\end{array}$ \\
\hline$C A D-24-5 C$ & $\begin{array}{l}\text { Criticality Alarm Detector. (Gama). } \\
\text { Detects radiation resulting from potential } \\
\text { critical ity excursions. Located in } 234-52 \text {, } \\
\text { Room } 1928 \text {. }\end{array}$ & $\begin{array}{l}\text { Nuclear Measurement Corp. } \\
\text { Model GA-2 TMO }\end{array}$ & $\begin{array}{l}H-2-28883 \text { sht } 1 \\
H-2-28883 \text { sht } 2 \\
H-2-28885 \text { sht } 8\end{array}$ \\
\hline$C A D-24-6 A$ & $\begin{array}{l}\text { Criticality Alarm Detector. (Gamma). } \\
\text { Detects radiation resulting from potential } \\
\text { criticality excursions. Located in } 234-52 \text {, } \\
\text { Room } 192 \text { C. }\end{array}$ & $\begin{array}{l}\text { Nucl ear Measurement Corp. } \\
\text { Model GA-2 TMO }\end{array}$ & $\begin{array}{l}H-2-28883 \text { sht } 1 \\
H-2-28883 \text { sht } 2 \\
H-2-28885 \text { sht } 8\end{array}$ \\
\hline$C A D-Z 4-6 B$ & $\begin{array}{l}\text { Criticality Alarm Detector. (Gamma). } \\
\text { Detects radiation resulting from potential } \\
\text { criticality excursions. Located in } 234-52 \text {, } \\
\text { Roon } 192 \text { C. }\end{array}$ & $\begin{array}{l}\text { Nuclear Measurement Corp. } \\
\text { Model GA-2 TMO }\end{array}$ & $\begin{array}{l}H-2-28883 \text { sht } 1 \\
H-2-28883 \text { sht } 2 \\
H-2-28885 \text { sht } 8\end{array}$ \\
\hline$C A D-24-6 C$ & $\begin{array}{l}\text { Criticality Alarm Detector. (Gamma). } \\
\text { Detects radiation resulting from potential } \\
\text { criticality excursions. Located in } 234-52 \text {, } \\
\text { Room } 192 \mathrm{C} \text {. }\end{array}$ & $\begin{array}{l}\text { Nuclear Measurement Corp. } \\
\text { Model GA-2 TMO }\end{array}$ & $\begin{array}{l}H-2-28883 \text { sht } 1 \\
H-2-28883 \text { sht } 2 \\
H-2-28885 \text { sht } 8\end{array}$ \\
\hline CADM $=24-1 A$ & $\begin{array}{l}\text { Criticality Alarm Detection Module. One (1) } \\
\text { per CAD. Provides dose rate indication, } \\
\text { calibration adjustments for the CAD-CADM } \\
\text { unit, and alarm functions. Located in } \\
\text { CAP- } 24 \text {. }\end{array}$ & $\begin{array}{l}\text { Nuclear Measurement Corp. } \\
\text { Model GA- } 2 \text { TMO }\end{array}$ & $\begin{array}{l}H-2-28883 \text { sht } 1 \\
H-2-28883 \text { sht } 2 \\
H-2-28885 \text { sht } 8\end{array}$ \\
\hline CADM-Z4-1B & $\begin{array}{l}\text { Criticality Alarm Detection Module. One (1) } \\
\text { per CAD. Provides dose rate indication, } \\
\text { cal ibration adjustments for the CAD-CADM } \\
\text { unit, and alarm functions. Located in } \\
\text { CAP-24. }\end{array}$ & $\begin{array}{l}\text { Nuclear Measurement Corp. } \\
\text { Model GA-2 TMO }\end{array}$ & $\begin{array}{l}H-2-28883 \text { sht } 1 \\
H-2-28883 \text { sht } 2 \\
\text { H-2-28885 sht } 8\end{array}$ \\
\hline CADM-Z4-1C & $\begin{array}{l}\text { Criticality Alarm Detection Module. One (1) } \\
\text { per CAD. Provides dose rate indication, } \\
\text { calibration adjustments for the CAD-CADH } \\
\text { unit, and alarm functions. Located in } \\
\text { CAP- } 24 \text {. }\end{array}$ & $\begin{array}{l}\text { Nuclear Measurement Corp. } \\
\text { Model GA-2 TMO }\end{array}$ & $\begin{array}{l}H-2-28883 \text { sht } 1 \\
H-2-28883 \text { sht } 2 \\
H-2-28885 \text { sht } 8\end{array}$ \\
\hline CADM-24-2A & $\begin{array}{l}\text { Criticality Alarm Detection Module. One (1) } \\
\text { per CAD. Provides dose rate indication, } \\
\text { calibration adjustments for the CAD-CADM } \\
\text { unit, and alarm functions. Located in } \\
\text { CAP-24. }\end{array}$ & $\begin{array}{l}\text { Nuclear Measurement Corp. } \\
\text { Model GA-2 TMO }\end{array}$ & $\begin{array}{l}H-2-28883 \text { sht } 1 \\
H-2-28883 \text { sht } 2 \\
H-2-28885 \text { sht } 8\end{array}$ \\
\hline CADM- $24-2 B$ & $\begin{array}{l}\text { Criticality Alarm Detection Module. One (1) } \\
\text { per CAD. Provides dose rate indication, } \\
\text { calibration adjustments for the CAD-CADM } \\
\text { unit, and alarm functions. Located in } \\
\text { CAP- } 24 \text {. }\end{array}$ & $\begin{array}{l}\text { Nuclear Measurement Corp. } \\
\text { Model GA-2 TMO }\end{array}$ & $\begin{array}{l}\text { H-2-28883 sht } 1 \\
H-2-28883 \text { sht } 2 \\
\text { H-2-28885 sht } 8\end{array}$ \\
\hline CADM- $24-2 C$ & $\begin{array}{l}\text { Criticality Alarm Detection Module. One (1) } \\
\text { per CAD. Provides dose rate indication, } \\
\text { calibration adjustments for the CAD-CADM } \\
\text { unit, and alarm functions. Located in } \\
\text { CAP-Z4. }\end{array}$ & $\begin{array}{l}\text { Nuclear Measurement Corp. } \\
\text { Model GA-2 TMO }\end{array}$ & $\begin{array}{l}H-2-28883 \text { sht } 1 \\
H-2-28883 \text { sht } 2 \\
H-2-28885 \text { sht } 8\end{array}$ \\
\hline
\end{tabular}




\begin{tabular}{|c|c|c|c|}
\hline$C A D M-24-3 A$ & $\begin{array}{l}\text { Criticality Alarm Detection Module. One ( } 1 \text { ) } \\
\text { per CAD. Provides dose rate indication, } \\
\text { cal ibration adjustments for the CAD-CADM } \\
\text { unit, and alarm functions. Located in } \\
\text { CAP- } 24 \text {. }\end{array}$ & $\begin{array}{l}\text { Nuclear Measurement Corp. } \\
\text { Model GA-2 TMO }\end{array}$ & $\begin{array}{l}H-2-28883 \text { sht } 1 \\
H-2-28883 \text { sht } 2 \\
H-2-28885 \text { sht } 8\end{array}$ \\
\hline CADM-24-3B & $\begin{array}{l}\text { Criticality Alarm Detection Module. One (1) } \\
\text { per CAD. Provides dose rate indication, } \\
\text { calibration adjustments for the CAD-CADM } \\
\text { unit, and alarm functions. Located in } \\
\text { CAP- } 24 \text {. }\end{array}$ & $\begin{array}{l}\text { Nuclear Measurement Corp. } \\
\text { Model GA-2 TMO }\end{array}$ & $\begin{array}{l}H-2-28883 \text { sht } 1 \\
H-2-28883 \text { sht } 2 \\
H-2-28885 \text { sht } 8\end{array}$ \\
\hline CADH- $24-3 C$ & $\begin{array}{l}\text { Criticality Alarm Detection Module. One (1) } \\
\text { per CAD. Provides dose rate indication, } \\
\text { calibration adjustments for the CAD-CADM } \\
\text { unit, and alarm functions. Located in } \\
\text { CAP- } 24 \text {. }\end{array}$ & $\begin{array}{l}\text { Nuclear Measurement Corp. } \\
\text { Model GA-2 TMO }\end{array}$ & $\begin{array}{ll}H-2-28883 & \text { sht } 1 \\
H-2-28883 & \text { sht } 2 \\
H-2-28885 & \text { sht } 8\end{array}$ \\
\hline CADM-Z4-4A & $\begin{array}{l}\text { Criticality Alarm Detection Module. One (1) } \\
\text { per CAD. Provides dose rate indication, } \\
\text { calibration adjustments for the CAD-CADM } \\
\text { unit, and alarm functions. Located in } \\
\text { CAP- } 24 \text {. }\end{array}$ & $\begin{array}{l}\text { Nuclear Measurement Corp. } \\
\text { Model GA-2 TMO }\end{array}$ & $\begin{array}{l}H-2-28883 \text { sht } 1 \\
H-2-28883 \text { sht } 2 \\
H-2-28885 \text { sht } 8\end{array}$ \\
\hline CADM- $24-4 B$ & $\begin{array}{l}\text { Criticality Alarm Detection Module. One (1) } \\
\text { per CAD. Provides dose rate indication, } \\
\text { calibration adjustments for the CAD-CADM } \\
\text { unit, and alarm functions. Located in } \\
\text { CAP- } 24 \text {. }\end{array}$ & $\begin{array}{l}\text { Nuclear Measurement Corp. } \\
\text { Model GA-2 TMO }\end{array}$ & $\begin{array}{l}H-2-28883 \text { sht } 1 \\
H-2-28883 \text { sht } 2 \\
H-2-28885 \text { sht } 8\end{array}$ \\
\hline$C A D M-24-4 C$ & $\begin{array}{l}\text { Criticality Alarm Detection Module. One (1) } \\
\text { per CAD. Provides dose rate indication, } \\
\text { calibration adjustments for the CAD-CADM } \\
\text { unit, and alarm functions. Located in } \\
\text { CAP-Z4. }\end{array}$ & $\begin{array}{l}\text { Nuclear Measurement Corp. } \\
\text { Model GA-2 TMO }\end{array}$ & $\begin{array}{l}H-2-28883 \text { sht } 1 \\
H-2-28883 \text { sht } 2 \\
H-2-28885 \text { sht } 8\end{array}$ \\
\hline CADM- $24-5 A$ & $\begin{array}{l}\text { Criticality Alarm Detection Module. One (1) } \\
\text { per CAD. Provides dose rate indication, } \\
\text { cal ibration adjustments for the CAD-CADM } \\
\text { unit, and alarm functions. Located in } \\
\text { CAP- } Z 4 \text {. }\end{array}$ & $\begin{array}{l}\text { Nuclear Measurement Corp. } \\
\text { Model GA-2 TMO }\end{array}$ & $\begin{array}{l}H-2-28883 \text { sht } 1 \\
H-2-28883 \text { sht } 2 \\
H-2-28885 \text { sht } 8\end{array}$ \\
\hline CADM $-24-5 B$ & $\begin{array}{l}\text { Criticality Alarm Detection Module. One ( } 1 \text { ) } \\
\text { per CAD. Provides dose rate indication, } \\
\text { cal ibration adjustments for the CAD-CADM } \\
\text { unit, and alarm functions. Located in } \\
\text { CAP-Z4. }\end{array}$ & $\begin{array}{l}\text { Nuclear Measurement Corp. } \\
\text { Model GA-2 TMO }\end{array}$ & $\begin{array}{l}h-2-28883 \text { sht } 1 \\
H-2-28883 \text { sht } 2 \\
H-2-28885 \text { sht } 8\end{array}$ \\
\hline CADM-24-5C & $\begin{array}{l}\text { Criticality Alarm Detection Module. One (1) } \\
\text { per CAD. Provides dose rate indication, } \\
\text { calibration adjustments for the CAD-CADH } \\
\text { unit, and alarm functions. Located in } \\
\text { CAP-Z4. }\end{array}$ & $\begin{array}{l}\text { Nuclear Measurement Corp. } \\
\text { Model GA-2 TMO }\end{array}$ & $\begin{array}{l}H-2-28883 \text { sht } 1 \\
H-2-28883 \text { sht } 2 \\
H-2-28885 \text { sht } 8\end{array}$ \\
\hline CADM-24-6A & $\begin{array}{l}\text { Criticality Alarm Detection Module. One (1) } \\
\text { per CAD. Provides dose rate indication, } \\
\text { calibration adjustments for the CAD-CADM } \\
\text { unit, and alarm functions. Located in } \\
\text { CAP-24. }\end{array}$ & $\begin{array}{l}\text { Nuclear Measurement Corp. } \\
\text { Model GA-2 TNO }\end{array}$ & $\begin{array}{l}H-2-28883 \text { sht } 1 \\
H-2-28883 \text { sht } 2 \\
H-2-28885 \text { sht } 8\end{array}$ \\
\hline CADM-24-68 & $\begin{array}{l}\text { Criticality Alarm Detection Module. One (1) } \\
\text { per CAD. Provides dose rate indication, } \\
\text { cal ibration adjustments for the CAD-CADM } \\
\text { unit, and alarm functions. Located in } \\
\text { CAP-Z4. }\end{array}$ & $\begin{array}{l}\text { Nuclear Measurement Corp. } \\
\text { Model GA-2 TMO }\end{array}$ & $\begin{array}{l}H-2-28883 \text { sht. } 1 \\
H-2-28883 \text { sht } 2 \\
H-2-28885 \text { sht } 8\end{array}$ \\
\hline CADM-24-6C & $\begin{array}{l}\text { Criticality Alarm Detection Module. One (1) } \\
\text { per CAD. Provides dose rate indication, } \\
\text { cal ibration adjustments for the CAD-CADM } \\
\text { unit, and alarm functions. Located in } \\
\text { CAP- } 24 \text {. }\end{array}$ & $\begin{array}{l}\text { Nuclear Measurement Corp. } \\
\text { Model GA-2 TMO }\end{array}$ & $\begin{array}{l}H-2-28883 \text { sht } 1 \\
H-2-28883 \text { sht } 2 \\
H-2-28885 \text { sht } 8\end{array}$ \\
\hline
\end{tabular}




\begin{tabular}{|c|c|c|c|}
\hline CAH-Z4-1 & $\begin{array}{l}\text { Criticality Alarm Horn. Located in Building } \\
234-52 \text { Room } 194 .\end{array}$ & $\begin{array}{l}\text { Audible Signaling Device: } \\
\text { Federal Signal Corp. Model } \\
300 \mathrm{GC}, 24 \text { VAC/DC Selectone } \\
\text { Connector Board: FS Corp. } \\
\text { Model } 300 \mathrm{CK} \\
\end{array}$ & $\begin{array}{l}H-2-28883 \text { sht } 1 \\
H-2-28883 \text { sht } 2 \\
H-2-28885 \text { sht } 8\end{array}$ \\
\hline CAH-Z4-2 & $\begin{array}{l}\text { Criticality Alarm Horn. Located in Building } \\
234-52 \text { Corridor } 292 \text {. }\end{array}$ & $\begin{array}{l}\text { Audible Signaling Device: } \\
\text { Federal Signal Corp. Model } \\
300 \mathrm{GC}, 24 \text { VAC/DC Selectone } \\
\text { Connector Board: FS Corp. } \\
\text { Hodel } 300 \text { CK }\end{array}$ & $\begin{array}{l}H-2-28883 \text { sht } 1 \\
H-2-28883 \text { sht } 2 \\
H-2-28885 \text { sht } 8\end{array}$ \\
\hline CAH-Z4-3 & $\begin{array}{l}\text { Criticality Alarm Horn. Located in Building } \\
234-52 \text { Room 192A. }\end{array}$ & $\begin{array}{l}\text { Audible Signaling Device: } \\
\text { Federal Signal Corp. Model } \\
300 \mathrm{GC}, 24 \text { VAC/DC Selectone } \\
\text { Connector Board: FS Corp. } \\
\text { Model } 300 \mathrm{CK}\end{array}$ & $\begin{array}{l}H-2-28883 \text { sht } 1 \\
H-2-28883 \text { sht } 2 \\
H-2-28885 \text { sht } 8\end{array}$ \\
\hline $\mathrm{CAH}-24-4$ & $\begin{array}{l}\text { Criticality Alarm Horn. Located in Building } \\
234-52 \text { Room 192B. }\end{array}$ & $\begin{array}{l}\text { Audible Signaling Device: } \\
\text { Federal Signal corp. Model } \\
300 \text { GC, } 24 \text { VACfDC Selectone } \\
\text { Connector Board: FS Corp. } \\
\text { Model } 300 \text { CK }\end{array}$ & $\begin{array}{l}\text { H-2-28883 sht } 1 \\
\text { H-2-28883 sht } 2 \\
H-2-28885 \text { sht } 8\end{array}$ \\
\hline $\mathrm{CAH}-24-5$ & $\begin{array}{l}\text { Criticality Alarm Horn. Located in Building } \\
234-5 Z \text { Room } 192 \mathrm{C} \text {. }\end{array}$ & $\begin{array}{l}\text { Audible Signal ing Device: } \\
\text { Federal Signal Corp. Model } \\
\text { 300 GC, } 24 \text { VAC/DC Selectone } \\
\text { Connector Board: FS Corp. } \\
\text { Model 300 CK }\end{array}$ & $\begin{array}{l}H-2-28883 \text { sht } 1 \\
H-2-28883 \text { sht } 2 \\
H-2-28885 \text { sht } 8\end{array}$ \\
\hline CAH-24-6 & $\begin{array}{l}\text { Criticality Alarm Horn. Located in Building } \\
234-52 \text { Room } 270 .\end{array}$ & $\begin{array}{l}\text { Audible signal ing Device: } \\
\text { Federal signal Corp. Model } \\
300 \text { GC, } 24 \text { VAC/DC Selectone } \\
\text { Connector Board: FS Corp. } \\
\text { Model } 300 \text { CK }\end{array}$ & $\begin{array}{l}H-2-28883 \text { sht } 1 \\
H-2-28883 \text { sht } 2 \\
H-2-28885 \text { sht } 9\end{array}$ \\
\hline $\mathrm{CAH}-\mathrm{Z4}-7$ & $\begin{array}{l}\text { Criticality Alarm Horn. Located in Building } \\
234-52 \text { Room } 183 .\end{array}$ & $\begin{array}{l}\text { Audible signal ing Device: } \\
\text { Federal signal Corp. Hodel } \\
300 \text { GC, } 24 \text { VAC/DC Selectone } \\
\text { Connector Board: FS Corp. } \\
\text { Model } 300 \text { CK }\end{array}$ & $\begin{array}{l}\text { H-2-28883 sht } 1 \\
\text { H-2-28883 sht } 2 \\
H-2-28885 \text { sht } 8\end{array}$ \\
\hline CAH-Z4-8 & $\begin{array}{l}\text { Criticality Atarm Horn. Located in Building } \\
234-52 \text { Corridor } 6 \text {. }\end{array}$ & $\begin{array}{l}\text { Audible signal ing Device: } \\
\text { Federal Signal Corp. Model } \\
\text { 300 GC, } 24 \text { VAC/DC Selectone } \\
\text { Connector Board: FS Corp. } \\
\text { Model } 300 \mathrm{CK}\end{array}$ & $\begin{array}{l}\text { H-2-28883 sht } 1 \\
\text { H-2-28883 sht } 2 \\
\text { H-2-28885 sht } 8\end{array}$ \\
\hline $\mathrm{CAH}-24-9$ & $\begin{array}{l}\text { Criticality Alarm Horn. Located in Building } \\
234-52 \text { Room } 321 .\end{array}$ & $\begin{array}{l}\text { Audible signal ing Device: } \\
\text { Federal Signal Corp. Model } \\
300 \text { GC, } 24 \text { VAC/DC Selectone } \\
\text { Connector Board: FS Corp. } \\
\text { Model } 300 \text { CK }\end{array}$ & $\begin{array}{l}\text { H-2-28883 sht } 1 \\
H-2-28883 \text { sht } 2 \\
H-2-28885 \text { sht } 10\end{array}$ \\
\hline $\mathrm{CAH}-\mathrm{Z4}-10$ & $\begin{array}{l}\text { Criticality Alarm Horn. Located in } \\
\text { Building } 234-52 \text { Corridor } 3 \text {. }\end{array}$ & $\begin{array}{l}\text { Audible signal ing Device: } \\
\text { Federal signal Corp. Model } \\
300 \text { GC, } 24 \text { VAC/DC Selectone } \\
\text { Connector Board: FS Corp. } \\
\text { Model } 300 \mathrm{CK}\end{array}$ & $\begin{array}{l}\mathrm{H}-2-28883 \text { sht } 1 \\
\mathrm{H}-2-28883 \text { sht } 2 \\
\mathrm{H}-2-28885 \text { sht } 10\end{array}$ \\
\hline CAH-Z4- 11 & $\begin{array}{l}\text { Criticality Alarm Horn. Located in } \\
\text { Building } 234-52 \text { Room } 308 \text {. }\end{array}$ & $\begin{array}{l}\text { Audible Signal ing Device: } \\
\text { Federal signal Corp. Model } \\
300 \text { GC, } 24 \text { VAC/DC Selectone } \\
\text { Connector Board: FS Corp. } \\
\text { Model } 300 \text { CK. } \\
\end{array}$ & $\begin{array}{l}\text { H-2-28883 sht } 1 \\
H-2-28883 \text { sht } 2 \\
H-2-28885 \text { sht } 10\end{array}$ \\
\hline $\mathrm{CAH}-\mathrm{Z} 4-12$ & $\begin{array}{l}\text { Criticality Alarm Horn. Located in Building } \\
234-52 \text { Room } 308 .\end{array}$ & $\begin{array}{l}\text { Audible Signal ing Device: } \\
\text { Federal Signal Corp. Model } \\
300 \text { GC, } 24 \text { VAC/OC Selectone } \\
\text { Connector Board: FS Corp. } \\
\text { Model } 300 \text { CK }\end{array}$ & $\begin{array}{l}\text { H-2-28883 sht } 1 \\
\text { H-2-28883 sht } 2 \\
\text { H-2-28885 sht } 10\end{array}$ \\
\hline
\end{tabular}




\begin{tabular}{|c|c|c|c|}
\hline $\mathrm{CAH}-\mathrm{Z4}-13$ & $\begin{array}{l}\text { Criticality Alarm Horn. Located in Building } \\
234-52 \text { Room } 308 \text {. }\end{array}$ & $\begin{array}{l}\text { Audible signal ing Device: } \\
\text { Federal signal Corp. Model } \\
300 \mathrm{GC}, 24 \text { VAC/DC Selectone } \\
\text { Connector Board: FS Corp. } \\
\text { Model } 300 \mathrm{CK}\end{array}$ & $\begin{array}{l}H-2-28883 \text { sht } 1 \\
H-2-28883 \text { sht } 2 \\
H-2-28885 \text { sht } 10\end{array}$ \\
\hline $\mathrm{CAH}-24-14$ & $\begin{array}{l}\text { Criticality Alarm Horn. Located in Building } \\
234-52 \text { Room } 308 .\end{array}$ & $\begin{array}{l}\text { Audible Signal ing Device: } \\
\text { Federal signal Corp. Model } \\
300 \text { GC, } 24 \text { VAC/DC Selectone } \\
\text { Connector Board: FS Corp. } \\
\text { Model } 300 \text { CK }\end{array}$ & $\begin{array}{l}H-2-28883 \text { sht } 1 \\
H-2-28883 \text { sht } 2 \\
H-2-28885 \text { sht } 10\end{array}$ \\
\hline CAH-Z4-15 & $\begin{array}{l}\text { Criticality Alarm Horn. Located in Building } \\
234-52 \text { Room } 341 \text {. }\end{array}$ & $\begin{array}{l}\text { Audible signal ing Device: } \\
\text { Federal signal corp. Model } \\
300 \mathrm{GC}, 24 \text { VAC/DC Selectone } \\
\text { Connector Board: FS Corp. } \\
\text { Model } 300 \mathrm{CK}\end{array}$ & $\begin{array}{l}H-2-28883 \text { sht } 1 \\
H-2-28883 \text { sht } 2 \\
H-2-28885 \text { sht } 10\end{array}$ \\
\hline CAH-24-16 & $\begin{array}{l}\text { Criticality Alarm Horn. Located in Building } \\
234-52 \text { Room 306A. }\end{array}$ & $\begin{array}{l}\text { Audible signaling Device: } \\
\text { Federal signal Corp. Model } \\
300 \mathrm{GC}, 24 \text { VAC/DC Selectone } \\
\text { Connector Board: FS .Corp. } \\
\text { Model } 300 \text { CK }\end{array}$ & $\begin{array}{l}H-2-28883 \text { sht } 1 \\
H-2-28883 \text { sht } 2 \\
H-2-28885 \text { sht } 10\end{array}$ \\
\hline $\mathrm{CAH}-\mathrm{Z4-17}$ & $\begin{array}{l}\text { Criticality Alarm Horn. Located in Building } \\
234-52 \text { Corridor } 1 .\end{array}$ & $\begin{array}{l}\text { Audible signal ing Device: } \\
\text { Federal signal Corp. Model } \\
300 \mathrm{GC}, 24 \text { VAC/DC Selectone } \\
\text { Connector Board: FS Corp. } \\
\text { Model } 300 \mathrm{CK}\end{array}$ & $\begin{array}{l}H-2-28883 \text { sht } 1 \\
H-2-28883 \text { sht } 2 \\
H-2-28885 \text { sht } 10\end{array}$ \\
\hline $\mathrm{CAH}-\mathrm{Z4}-18$ & $\begin{array}{l}\text { Criticality Alarm Horn. Located outside on } \\
\text { North Wall of Building } 234-52 \text { (near entrance } \\
\text { to } R m \text { 192). }\end{array}$ & $\begin{array}{l}\text { Audible signal ing Device; } \\
\text { Federal signal Corp. Model } \\
300 \text { GC, } 24 \text { VAC/DC Selectone } \\
\text { Connector Board: FS Corp. } \\
\text { Model } 300 \text { CK }\end{array}$ & $\begin{array}{l}\text { H-2-28883 sht } 1 \\
\text { H-2-28883 sht } 2 \\
\text { H-2-28885 sht } 8\end{array}$ \\
\hline CAH-24-19 & $\begin{array}{l}\text { Criticality Alarm Horn. Located outside in } \\
\text { Stairway 13, Southwest Corner of Building } \\
234-52 \text {. }\end{array}$ & $\begin{array}{l}\text { Audible Signaling Device: } \\
\text { Federal Signal Corp. Model } \\
300 \mathrm{GC}, 24 \text { VAC/OC Selectone } \\
\text { Connector Board: FS Corp. } \\
\text { Model } 300 \mathrm{CK} \\
\end{array}$ & $\begin{array}{l}H-2-28883 \text { sht } 1 \\
H-2-28883 \text { sht } 2 \\
H-2-28885 \text { sht } 8\end{array}$ \\
\hline $\mathrm{CAH}-24-20$ & $\begin{array}{l}\text { Criticality Alarm Horn. Located outside on } \\
\text { West Wall (Southwest Corner) of Building } \\
234-52 \text {. }\end{array}$ & $\begin{array}{l}\text { Audible signaling Device: } \\
\text { Federal signal Corp. Model } \\
300 \text { GC, } 24 \text { VAC/DC Selectone } \\
\text { Connector Board: FS Corp. } \\
\text { Model } 300 \text { CK }\end{array}$ & $\begin{array}{l}H-2-28883 \text { sht } 1 \\
H-2-28883 \text { sht } 2 \\
H-2-28885 \text { sht } 8\end{array}$ \\
\hline $\mathrm{CAH}-\mathrm{Z4}-21$ & $\begin{array}{l}\text { Criticality Alarn horn. Located in Building } \\
234-52 \text { Roon } 265 \text {. }\end{array}$ & $\begin{array}{l}\text { Audible signaling Device: } \\
\text { Federal signal Corp. Model } \\
300 \text { GC, } 24 \text { VAC/DC Selectone } \\
\text { Connector Board: FS Corp. } \\
\text { Model } 300 \text { CK }\end{array}$ & $\begin{array}{l}H-2-28883 \text { sht } 1 \\
H-2-28883 \text { sht } 2 \\
H-2-28885 \text { sht } 9\end{array}$ \\
\hline $\mathrm{CAH}-\mathrm{Z4}-22$ & $\begin{array}{l}\text { Criticality Alarm Horn. Located in Building } \\
234-52 \text { Room } 2350 \text {. }\end{array}$ & $\begin{array}{l}\text { Audible signaling Device: } \\
\text { Federal Signal Corp. Model } \\
300 \text { GC, } 24 \text { VAC/DC Selectone } \\
\text { Connector Board: FS Corp. } \\
\text { Model } 300 \text { CK }\end{array}$ & $\begin{array}{l}H-2-28883 \text { sht } 1 \\
H-2-28883 \text { sht } 2 \\
H-2-28885 \text { sht } 8\end{array}$ \\
\hline $\mathrm{CAH}-\mathrm{Z} 4-23$ & $\begin{array}{l}\text { Criticality Alarm Horn. Located on North } \\
\text { West Roof of } 234-5 Z \text {. }\end{array}$ & $\begin{array}{l}\text { Audible signaling Device: } \\
\text { Federal signal Corp. Model } \\
300 \mathrm{GC}, 24 \text { VAC/DC Selectone } \\
\text { Connector Board: FS Corp. } \\
\text { Model } 300 \text { CK }\end{array}$ & $\begin{array}{l}\text { H-2-28883 sht } 1 \\
\text { H-2-28883 sht } 2 \\
\text { H-2-28885 sht } 11\end{array}$ \\
\hline $\mathrm{CAH}-\mathrm{Z} 4-24$ & $\begin{array}{l}\text { Criticality Alam Horn. Located on West } \\
\text { Roof of } 234-5 z \text {. }\end{array}$ & $\begin{array}{l}\text { Audible Signaling Device: } \\
\text { Federal signal Corp. Model } \\
300 \text { GC, } 24 \text { VAC/DC Selectone } \\
\text { Connector Board: FS Corp. } \\
\text { Model } 300 \text { CK }\end{array}$ & $\begin{array}{l}H-2-28883 \text { sht } 1 \\
H-2-28883 \text { sht } 2 \\
H-2-28885 \text { sht } 11\end{array}$ \\
\hline
\end{tabular}




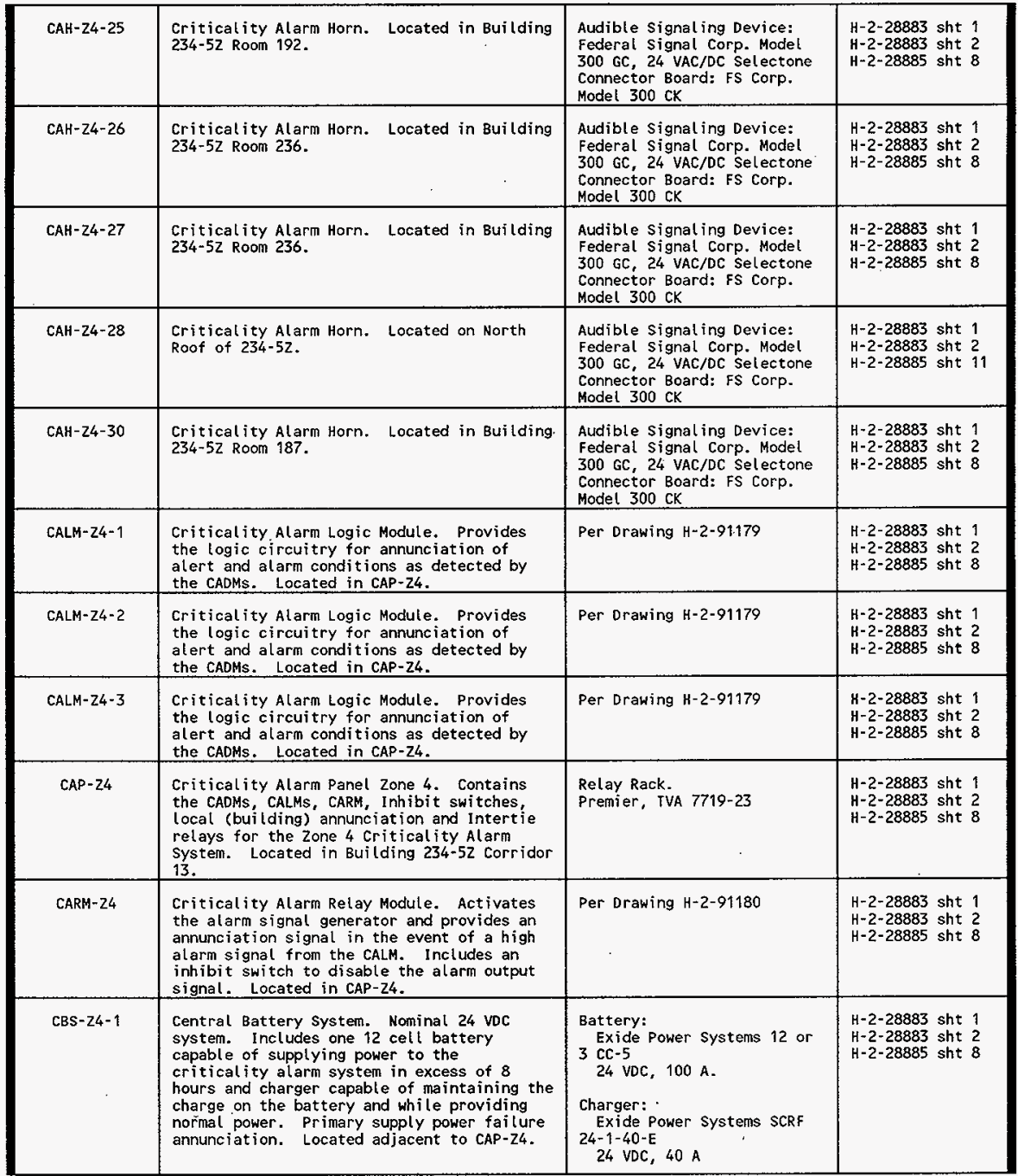




\begin{tabular}{|c|c|c|c|}
\hline $\begin{array}{l}\text { AC POWER } \\
\text { RELAY } \\
\text { (Not required } \\
\text { for labeling } \\
\text { at this } \\
\text { time.) } \\
\end{array}$ & $\begin{array}{l}\text { (SC-3 SE) AC Power Monitor Relay. } \\
\text { Deenergizes on loss of power. A manual } \\
\text { reset is required to prevent a fluctuating } \\
\text { (erratic) power supply from initiating false } \\
\text { alarms. }\end{array}$ & Allen Bradley \# 700-c201A1 & $\begin{array}{l}H-2-28883 \text { sht } 1 \\
H-2-28883 \text { sht } 2 \\
H-2-28885 \text { sht } 8\end{array}$ \\
\hline$S-4-1 A$ & $\begin{array}{l}\text { Inhibit Switch for CAD/CADH-Z4-1A used to } \\
\text { prevent annunciation of alarm during test, } \\
\text { calibration, and replacement of CADS and } \\
\text { CADMs. Located on CAP-Z4. }\end{array}$ & $\begin{array}{l}\text { Square D Class } 9001 \text {, } \\
\text { Type LRSA-2K1 with } \\
\text { rype LA-1 Contact Blocks }\end{array}$ & $\begin{array}{l}H-2-28883 \text { sht } 1 \\
H-2-28883 \text { sht } 2 \\
H-2-28885 \text { sht } 8\end{array}$ \\
\hline$s-4-1 B$ & $\begin{array}{l}\text { Inhibit Switch for CAD/CADM-Z4-1B used to } \\
\text { prevent annunciat ion of alarm during test, } \\
\text { calibration, and replacement of CADS and } \\
\text { CADMs. Located on CAP-24. }\end{array}$ & $\begin{array}{l}\text { Square } 0 \text { Class } 9001 \text {, } \\
\text { Type LRSA-2K1 with } \\
\text { Type LA-1 Contact Blocks }\end{array}$ & $\begin{array}{l}H-2-28883 \text { sht } 1 \\
H-2-28883 \text { sht } 2 \\
H-2-28885 \text { sht } 8\end{array}$ \\
\hline$S-4-2 A$ & $\begin{array}{l}\text { Inhibit Switch for CAD/CADM-24-2A used to } \\
\text { prevent annunciation of alarm during test, } \\
\text { calibration, and replacement of CADS and } \\
\text { CADMS. Located on CAP-Z4. }\end{array}$ & $\begin{array}{l}\text { Square } 0 \quad \text { Class } 9001 \text {, } \\
\text { Type LRSA-2K1 with } \\
\text { Type LA-1 Contact Blocks }\end{array}$ & $\begin{array}{l}H-2-28883 \text { sht } 1 \\
H-2-28883 \text { sht } 2 \\
H-2-28885 \text { sht } 8\end{array}$ \\
\hline$S-4-2 B$ & $\begin{array}{l}\text { Inhibit Switch for CAD/CADM- } 24-28 \text { used to } \\
\text { prevent annunciation of alarm during test, } \\
\text { calibration, and replacement of CADs and } \\
\text { CADMS. Located on CAP-Z4. }\end{array}$ & $\begin{array}{l}\text { Square D Class } 9001 \text {, } \\
\text { Type LRSA-2K1 with } \\
\text { Type LA-1 Contact Blocks }\end{array}$ & $\begin{array}{l}H-2-28883 \text { sht } 1 \\
H-2-28883 \text { sht } 2 \\
H-2-28885 \text { sht } 8\end{array}$ \\
\hline$S-4-3 B$ & $\begin{array}{l}\text { Inhibit switch for CAD/CADM-24-3B used to } \\
\text { prevent annunciation of alarm during test, } \\
\text { calibration, and replacement of CADS and } \\
\text { CADMs. Located on CAP-Z4. }\end{array}$ & $\begin{array}{l}\text { Square D Class } 9001 \text {, } \\
\text { Type LRSA-2K1 with } \\
\text { Type LA-1 Contact Blocks }\end{array}$ & $\begin{array}{l}\text { H-2-28883 sht } 1 \\
\text { H-2-28883 sht } 2 \\
\text { H-2-28885 sht } 8\end{array}$ \\
\hline$s-4-3 c$ & $\begin{array}{l}\text { Inhibit Switch for CAD/CADM-24-3C used to } \\
\text { prevent annunciation of alarm during test, } \\
\text { calibration, and replacement of CADS and } \\
\text { CADMs. Located on CAP- } 24 \text {. }\end{array}$ & $\begin{array}{l}\text { Square D Class } 9001 \text {, } \\
\text { Type LRSA-2K1 with } \\
\text { Type LA- } 1 \text { Contact Blocks }\end{array}$ & $\begin{array}{l}H-2-28883 \text { sht } 1 \\
H-2-28883 \text { sht } 2 \\
H-2-28885 \text { sht } 8\end{array}$ \\
\hline$s-4-4 A$ & $\begin{array}{l}\text { Inhibit Switch for CAD/CADM-Z4-4A used to } \\
\text { prevent annunciation of alarm during test, } \\
\text { calibration, and replacement of CADS and } \\
\text { CADMs. Located on CAP-24. }\end{array}$ & $\begin{array}{l}\text { Square D Class } 9001 \text {, } \\
\text { Type LRSA-2Ki with } \\
\text { Type LA-1 Contact Blocks }\end{array}$ & $\begin{array}{l}\text { H-2-28883 sht } 1 \\
\text { H-2-28883 sht } 2 \\
H-2-28885 \text { sht } 8\end{array}$ \\
\hline
\end{tabular}




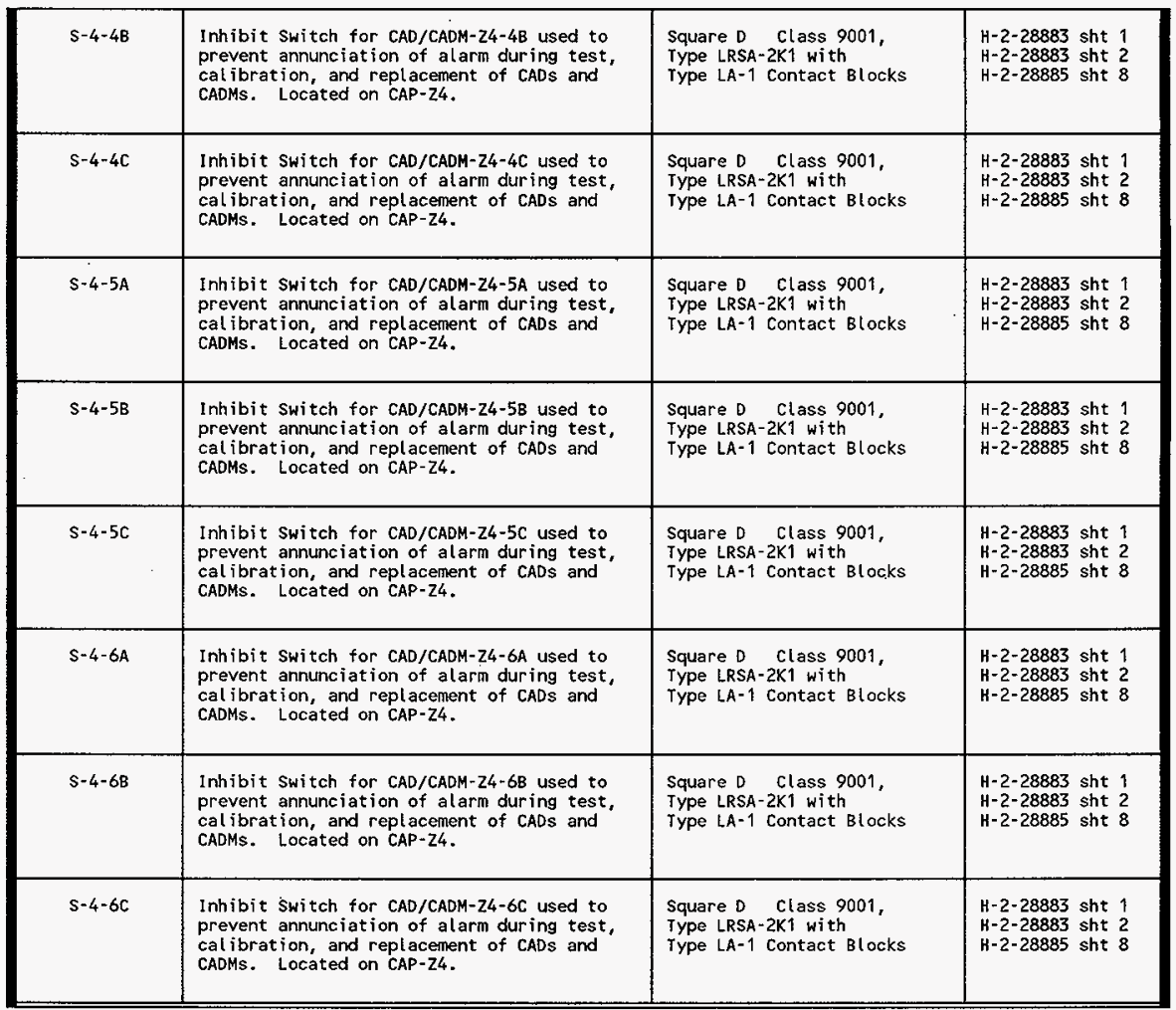




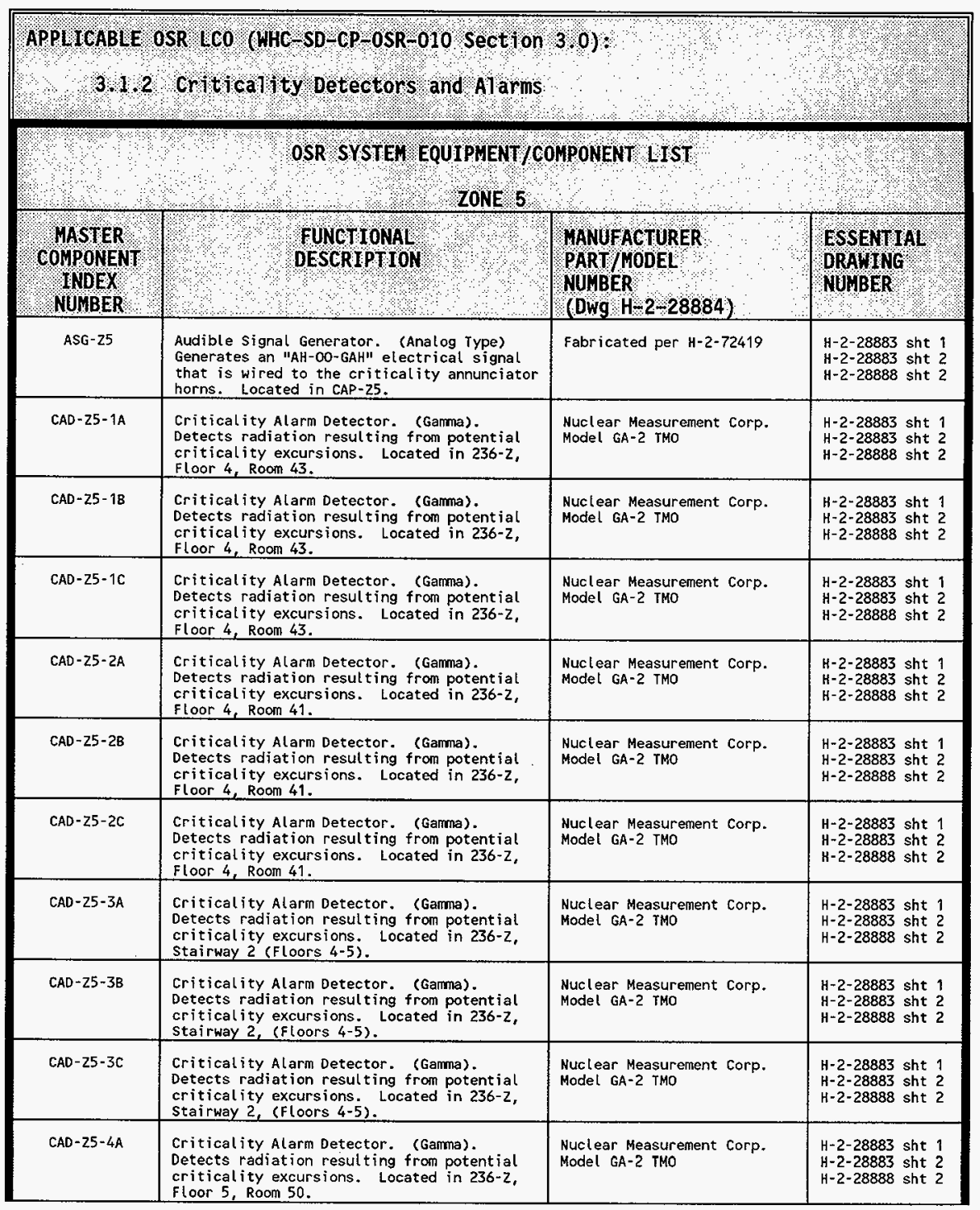




\begin{tabular}{|c|c|c|c|}
\hline \multicolumn{4}{|c|}{ 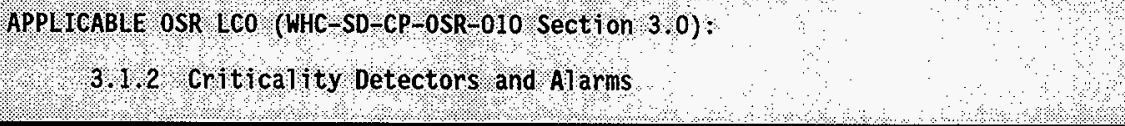 } \\
\hline & $\begin{array}{r}\text { OSR SYSTEN EQUTPHEN } \\
\text { ZONE }\end{array}$ & PONENT LIST & \\
\hline $\begin{array}{l}\text { MASTER } \\
\text { COMPONENT } \\
\text { INDEX } \\
\text { NUMBER }\end{array}$ & DOESCRIPION & $\begin{array}{l}\text { MANUFACTURER } \\
\text { PART/MODEL } \\
\text { NUMBER } \\
\text { (DWGH } 2-28884)\end{array}$ & $\begin{array}{l}S S S E N A \\
\text { DRALING }\end{array}$ \\
\hline$C A D-25-4 C$ & $\begin{array}{l}\text { Criticality Alarm Detector. (Gamma). } \\
\text { Detects radiation resulting from potential } \\
\text { criticality excursions. Located in } 236-2 \text {, } \\
\text { Floor } 5 \text {, Room } 50 \text {. }\end{array}$ & $\begin{array}{l}\text { Nuclear Measurement Corp. } \\
\text { Model GA-2 TMO }\end{array}$ & $\begin{array}{l}H-2-28883 \text { sht } 1 \\
H-2-28883 \text { sht } 2 \\
H-2-28888 \text { sht } 2\end{array}$ \\
\hline$C A D-25-5 A$ & $\begin{array}{l}\text { Criticality Alarm Detector. (Gamma). } \\
\text { Detects radiation resulting from potential } \\
\text { criticality excursions. Located in } 236-2 \text {, } \\
\text { Floor } 3 \text {, Elevator. }\end{array}$ & $\begin{array}{l}\text { Nuclear Measurement Corp. } \\
\text { Model GA-2 TMO }\end{array}$ & $\begin{array}{l}H-2-28883 \text { sht } 1 \\
H=2-28883 \text { sht } 2 \\
H-2-28888 \text { sht } 2\end{array}$ \\
\hline$C A D-25-5 B$ & $\begin{array}{l}\text { Criticality Alarm Detector. (Gamma). } \\
\text { Detects radiation resulting from potential } \\
\text { criticality excursions. Located in } 236-z_{1} \\
\text { Floor } 3 \text {, Elevator. }\end{array}$ & $\begin{array}{l}\text { Nuclear Measurement corp. } \\
\text { Model GA-2 TMO }\end{array}$ & $\begin{array}{l}H-2-28883 \text { sht } 1 \\
H-2-28883 \text { sht } 2 \\
H-2-28888 \text { sht } 2\end{array}$ \\
\hline$C A D-25-6 B$ & $\begin{array}{l}\text { Criticality Alarm Detector. (Gamma). } \\
\text { Detects radiation resulting from potential } \\
\text { criticality excursions. Located in } 236-Z \text {, } \\
\text { Floor } 3 \text {, Room 12, Maintenance Hood. }\end{array}$ & $\begin{array}{l}\text { Nuclear Measurement Corp. } \\
\text { Model GA-2 TMO }\end{array}$ & $\begin{array}{l}H-2-28883 \text { sht } 1 \\
H-2-28883 \text { sht } 2 \\
H-2-28888 \text { sht } 2\end{array}$ \\
\hline$C A D-25-6 C$ & $\begin{array}{l}\text { Criticality Alarm Detector. (Garma). } \\
\text { Detects radiation resulting from potential } \\
\text { criticality excursions. Located in 236-z, } \\
\text { Floor } 3 \text {, Room } 12 \text {, Maintenance Hood. }\end{array}$ & $\begin{array}{l}\text { Nuclear Measurement Corp. } \\
\text { Model GA-2 TMO }\end{array}$ & $\begin{array}{l}H-2-28883 \text { sht } 1 \\
H-2-28883 \text { sht } 2 \\
H-2-28888 \text { sht } 2\end{array}$ \\
\hline$C A D-Z 5-7 A$ & $\begin{array}{l}\text { Critical ity Alarm Detector. (Gamma). } \\
\text { Detects radiation resulting from potential } \\
\text { criticality excursions. Located in } 236-2 \text {, } \\
\text { Floor } 2 \text {, Room } 26 \text {. }\end{array}$ & $\begin{array}{l}\text { Nuclear Measurement Corp. } \\
\text { Model GA-2 IMO }\end{array}$ & $\begin{array}{l}H-2-28883 \text { sht } 1 \\
H-2-28883 \text { sht } 2 \\
H-2-28888 \text { sht } 2\end{array}$ \\
\hline$C A D-Z 5-7 B$ & $\begin{array}{l}\text { Critical ity Alarm Detector. (Gamma). } \\
\text { Detects radiation resulting from potential } \\
\text { criticality excursions. Located in } 236-z \text {, } \\
\text { Floor } 2 \text {, Room } 26 \text {. }\end{array}$ & $\begin{array}{l}\text { Nuclear Measurement Corp. } \\
\text { Model GA-2 TMO }\end{array}$ & $\begin{array}{l}H-2-28883 \text { sht } 1 \\
H-2-28883 \text { sht } 2 \\
H-2-28888 \text { sht } 2\end{array}$ \\
\hline$C A D-25-7 C$ & $\begin{array}{l}\text { Criticality Alarm Detector. (Gamma). } \\
\text { Detects radiation resulting from potential } \\
\text { criticality excursions. Located in } 236-2 \text {, } \\
\text { Floor } 2 \text {, Room } 26 \text {. }\end{array}$ & $\begin{array}{l}\text { Nuclear Measurement Corp. } \\
\text { Model GA-2 TMO }\end{array}$ & $\begin{array}{l}\text { H-2-28883 sht } 1 \\
\text { H-2-28883 sht } 2 \\
\text { H-2-28888 sht } 2\end{array}$ \\
\hline
\end{tabular}




\begin{tabular}{|c|c|c|c|}
\hline 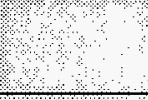 & 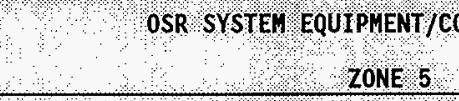 & MPONENT LIST & \\
\hline $\begin{array}{l}\text { COMASER } \\
\text { COMONENT } \\
\text { NUDEX } \\
\text { NUER }\end{array}$ & 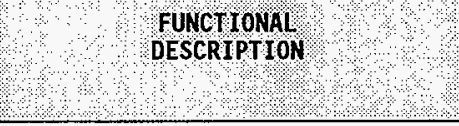 & $\begin{array}{l}\text { WANUFACTURER } \\
\text { PART/NODEL } \\
\text { NUNBER } \\
\text { (DWg } \mathrm{H}-2-28884)\end{array}$ & $\begin{array}{l}\text { ESSENTHL } \\
\text { DRAWING } \\
\text { NUNBER }\end{array}$ \\
\hline CAOM-Z5- 18 & $\begin{array}{l}\text { Criticality Alarm Detection Module. One (1) } \\
\text { per CAD. Provides dose rate indication, } \\
\text { calibration adjustments for the CAD-CADM } \\
\text { unit, and alarm functions. Located in } \\
\text { CAP- } 25 \text {. }\end{array}$ & $\begin{array}{l}\text { Nuclear Measurement Corp. } \\
\text { Model GA-2 TMO }\end{array}$ & $\begin{array}{l}H-2-28883 \text { sht } 1 \\
H-2-28883 \text { sht } 2 \\
H-2-28888 \text { sht } 2\end{array}$ \\
\hline CADM- $25-1 C$ & $\begin{array}{l}\text { Criticality Alarm Detection Module. One (1) } \\
\text { per CAD. Provides dose rate indication, } \\
\text { calibration adjustments for the CAD-CADM } \\
\text { unit, and alarm functions. Located in } \\
\text { CAP-25. }\end{array}$ & $\begin{array}{l}\text { Nucl ear Measurement Corp. } \\
\text { Model GA-2 TMO }\end{array}$ & $\begin{array}{l}H-2-28883 \text { sht } 1 \\
H-2-28883 \text { sht } 2 \\
H-2-28888 \text { sht } 2\end{array}$ \\
\hline CAOM-25-2A & $\begin{array}{l}\text { Criticality Alarm Detection Module. One (1) } \\
\text { per CAD. Provides dose rate indication, } \\
\text { calibration adjustments for the CAD-CADM } \\
\text { unit, and alarm functions. Located in } \\
\text { CAP-25. }\end{array}$ & $\begin{array}{l}\text { Nucl ear Measurement Corp. } \\
\text { Model GA-2 TMO }\end{array}$ & $\begin{array}{l}H-2-28883 \text { sht } 1 \\
H-2-28883 \text { sht } 2 \\
H-2-28888 \text { sht } 2\end{array}$ \\
\hline CADM- $25-2 C$ & $\begin{array}{l}\text { Criticality Alarm Detection Module. One (1) } \\
\text { per CAD. Provides dose rate indication, } \\
\text { cal ibration adjustments for the CAD-CADM } \\
\text { Unit, and alarm functions. Located in } \\
\text { CAP-25. }\end{array}$ & $\begin{array}{l}\text { Nuclear Measurement Corp. } \\
\text { Model GA-2 TMO }\end{array}$ & $\begin{array}{l}H-2-28883 \text { sht } 1 \\
H-2-28883 \text { sht } 2 \\
H-2-28888 \text { sht } 2\end{array}$ \\
\hline CADM- $25-3 A$ & $\begin{array}{l}\text { Criticality Alarm Detection Module. One (1) } \\
\text { per CAD. Provides dose rate indication, } \\
\text { calibration adjustments for the CAD-CADM } \\
\text { unit, and alarm functions. Located in } \\
\text { CAP-25. }\end{array}$ & $\begin{array}{l}\text { Nuclear Measurement Corp. } \\
\text { Model GA-2 TMO }\end{array}$ & $\begin{array}{l}H-2-28883 \text { sht } 1 \\
H-2-28883 \text { sht } 2 \\
H-2-28888 \text { sht } 2\end{array}$ \\
\hline CADN-Z5-3B & $\begin{array}{l}\text { Criticality Alarm Detection Module. One (1) } \\
\text { per CAD. Provides dose rate indication, } \\
\text { calibration adjustments for the CAD-CADM } \\
\text { unit, and alarm functions. Located in } \\
\text { CAP- } Z 5 \text {. }\end{array}$ & $\begin{array}{l}\text { Nuclear Measurement corp. } \\
\text { Model GA-2 TMO }\end{array}$ & $\begin{array}{l}H-2-28883 \text { sht } 1 \\
H-2-28883 \text { sht } 2 \\
H-2-28888 \text { sht } 2\end{array}$ \\
\hline$C A D M-25-3 C$ & $\begin{array}{l}\text { Criticality Alarm Detection Module. One ( } 1 \text { ) } \\
\text { per CAD. Provides dose rate indication, } \\
\text { calibration adjustments for the CAD-CADM } \\
\text { unit, and alarm functions. Located in } \\
\text { CAP- } 25 \text {. }\end{array}$ & $\begin{array}{l}\text { Nuclear Measurement Corp. } \\
\text { Model GA-2 TMO }\end{array}$ & $\begin{array}{l}H-2-28883 \text { sht } 1 \\
H-2-28883 \text { sht } 2 \\
H-2-28888 \text { sht } 2\end{array}$ \\
\hline
\end{tabular}




\begin{tabular}{|c|c|c|c|}
\hline \multicolumn{4}{|c|}{ 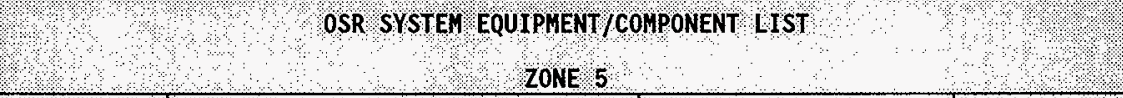 } \\
\hline $\begin{array}{l}\text { WASTER } \\
\text { QOMPONENT } \\
\text { NONDEX } \\
\text { NUMBER }\end{array}$ & ODESCRIPTION & $\begin{array}{l}\text { MANUPACTURER } \\
\text { PARE } \\
\text { AUPBEDEE } \\
\text { (DWg H-2_28884) }\end{array}$ & $\begin{array}{l}\text { ESSENTAL } \\
\text { DRAMING } \\
\text { HEMBER }\end{array}$ \\
\hline $\mathrm{CADM}-25-4 \mathrm{~B}$ & $\begin{array}{l}\text { Criticality Alarm Detection Module. One (1) } \\
\text { per CAD. Provides dose rate indication, } \\
\text { cal ibration adjustments for the CAD-CADM } \\
\text { unit, and alarm functions. Located in } \\
\text { CAP- } 25 \text {. }\end{array}$ & $\begin{array}{l}\text { Nucl ear Neasurement Corp. } \\
\text { Model GA-2 TMO }\end{array}$ & $\begin{array}{l}H-2-28883 \text { sht } 1 \\
H-2-28883 \text { sht } 2 \\
H-2-28888 \text { sht } 2\end{array}$ \\
\hline$C A D M-25-4 C$ & $\begin{array}{l}\text { Criticality Alarm Detection Module, One (1) } \\
\text { per CAD. Provides dose rate indication, } \\
\text { cal ibration adjustments for the CAD-CADM } \\
\text { unit, and alarm functions. Located in } \\
\text { CAP-Z5. }\end{array}$ & $\begin{array}{l}\text { Nuclear Measurement Corp. } \\
\text { Model GA-2 THO }\end{array}$ & $\begin{array}{l}H-2-28883 \text { sht } 1 \\
H-2-28883 \text { sht } 2 \\
H-2-28888 \text { sht } 2\end{array}$ \\
\hline$C A D M-25-5 A$ & $\begin{array}{l}\text { Criticality Alarm Detection Module. One (1) } \\
\text { per CAD. Provides dose rate indication, } \\
\text { cal ibration adjustments for the CAD-CADM } \\
\text { unit, and alarm functions. Located in } \\
\text { CAP- } 25 \text {. }\end{array}$ & $\begin{array}{l}\text { Nuclear Measurement Corp. } \\
\text { Model GA-2 INO }\end{array}$ & $\begin{array}{l}H-2-28883 \text { sht } 1 \\
H-2-28883 \text { sht } 2 \\
H-2-28888 \text { sht } 2\end{array}$ \\
\hline CADM- $25-5 \mathrm{C}$ & $\begin{array}{l}\text { Critical ity Alarm Detection Module. One (1) } \\
\text { per CAD. Provides dose rate indication, } \\
\text { calibration adjustments for the CAD-CADM } \\
\text { unit, and alarm functions. Located in } \\
\text { CAP-ZS. }\end{array}$ & $\begin{array}{l}\text { Nucl ear Measurement Corp. } \\
\text { Model GA-2 THO }\end{array}$ & $\begin{array}{l}H-2-28883 \text { sht } 1 \\
H-2-28883 \text { sht } 2 \\
H-2-28888 \text { sht } 2\end{array}$ \\
\hline CADM- $25-6 A$ & $\begin{array}{l}\text { Criticality Alarm Detection Module. One (1) } \\
\text { per CAD. Provides dose rate indication, } \\
\text { cal ibration adjustments for the CAD-CADM } \\
\text { unit, and alarm functions. Located in } \\
\text { CAP- } 25 \text {. }\end{array}$ & $\begin{array}{l}\text { Nuclear Measurement Corp. } \\
\text { Model GA-2 TMO }\end{array}$ & $\begin{array}{l}H-2-28883 \text { sht } 1 \\
H-2-28883 \text { sht } 2 \\
H-2-28888 \text { sht } 2\end{array}$ \\
\hline CADM- $25-6 B$ & $\begin{array}{l}\text { Criticality Alarm Detection Module. One (1) } \\
\text { per CAD. Provides dose rate indication, } \\
\text { cal ibration adjustments for the CAD-CADM } \\
\text { unit, and alarm functions. Located in } \\
\text { CAP-Z5. }\end{array}$ & $\begin{array}{l}\text { Nucl ear Measurement Corp. } \\
\text { Model GA-2 TMO }\end{array}$ & $\begin{array}{l}H-2-28883 \text { sht } 1 \\
H-2-28883 \text { sht } 2 \\
H-2-28888 \text { sht } 2\end{array}$ \\
\hline CADM-25-6C & $\begin{array}{l}\text { Criticality Alarm Detection Module. One (1) } \\
\text { per CAD. Provides dose rate indication, } \\
\text { cal ibration adjustments for the CAD-CADM } \\
\text { unit, and alarm functions. Located in } \\
\text { CAP- } 25 \text {. }\end{array}$ & $\begin{array}{l}\text { Nuclear Measurement Corp. } \\
\text { Model GA-2 TMO }\end{array}$ & $\begin{array}{l}H-2-28883 \text { sht } 1 \\
H-2-28883 \text { sht } 2 \\
H-2-28888 \text { sht } 2\end{array}$ \\
\hline
\end{tabular}




\begin{tabular}{|c|c|c|c|}
\hline MPPLABEOS & \multicolumn{2}{|c|}{ 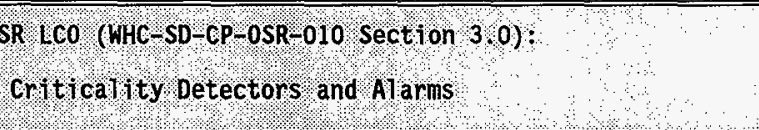 } & $\therefore \quad r$ \\
\hline & 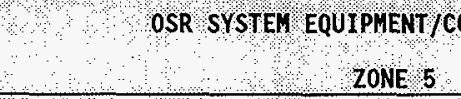 & & \\
\hline $\begin{array}{l}\text { MASTER } \\
\text { ONPONENT } \\
\text { WNDEX } \\
\text { NUMBER }\end{array}$ & 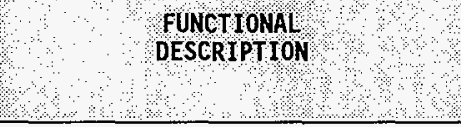 & $\begin{array}{l}\text { WANUFACTURER } \\
\text { PART } \\
\text { NUNBDER } \\
\text { (DWG H } 2-28884 \text { ) }\end{array}$ & $\begin{array}{l}\text { ESSENALE } \\
\text { DRAWIG } \\
\text { NUWBER }\end{array}$ \\
\hline CADM- $25-7 A$ & $\begin{array}{l}\text { Criticality Alarm Detection Module. One (1) } \\
\text { per CAD. Provides dose rate indication, } \\
\text { cal ibration adjustments for the CAD-CADM } \\
\text { unit, and alarm functions. Located in } \\
\text { CAP-25. }\end{array}$ & $\begin{array}{l}\text { Nuclear Measurement corp. } \\
\text { Model GA-2 TMO }\end{array}$ & $\begin{array}{l}H-2-28883 \text { sht } 1 \\
H-2-28883 \text { sht } 2 \\
H-2-28888 \text { sht } 2\end{array}$ \\
\hline CADM $-25-7 B$ & $\begin{array}{l}\text { Criticality Alarm Detection Module. One (1) } \\
\text { per CAD. Provides dose rate indication, } \\
\text { cal ibration adjustments for the CAD-CADM } \\
\text { unit, and alarm functions. Located in } \\
\text { CAP-Z5. }\end{array}$ & $\begin{array}{l}\text { Nuclear Measurement corp. } \\
\text { Model GA-2 TMO }\end{array}$ & $\begin{array}{l}H-2-28883 \text { sht } 1 \\
H-2-28883 \text { sht } 2 \\
H-2-28888 \text { sht } 2\end{array}$ \\
\hline CADM-25-7C & $\begin{array}{l}\text { Criticality Alarm Detection Module. One (1) } \\
\text { per CAD. Provides dose rate indication, } \\
\text { cal ibration adjustment's for the CAD-CADM } \\
\text { unit; and alarm functions. Located in } \\
\text { CAP-ż. }\end{array}$ & $\begin{array}{l}\text { Nuclear Measurement Corp. } \\
\text { Model GA-2 TMO }\end{array}$ & $\begin{array}{l}H-2-28883 \text { sht } ? \\
H-2-28883 \text { sht } 2 \\
H-2-28888 \text { sht } 2\end{array}$ \\
\hline $\mathrm{CAH}-25-1$ & $\begin{array}{l}\text { Criticality Alarm Horn. Located in Building } \\
236-2 \text {, Floor } 6 \text {, Room } 60 \text {. }\end{array}$ & $\begin{array}{l}\text { Audible signaling Device: } \\
\text { Federal signal Corp. Model } \\
300 \mathrm{GC}, 24 \text { VAC/DC Selectone } \\
\text { Connector } 8 \text { oard: FS Corp. } \\
\text { Model } 300 \text { CK }\end{array}$ & $\begin{array}{l}H-2-28883 \text { sht } 1 \\
H-2-28883 \text { sht } 2 \\
H-2-28888 \text { sht } 2\end{array}$ \\
\hline $\mathrm{CAH}-25-2$ & $\begin{array}{l}\text { Criticality Alarm Horn. Located in Building } \\
236-2 \text {, Stairway } 2 \text {, Floor } 5 \text {. }\end{array}$ & $\begin{array}{l}\text { Audible Signal ing Device: } \\
\text { Federal Signal Corp. Model } \\
300 \text { GC, } 24 \text { VAC/OC Selectone } \\
\text { Connector Board: FS Corp. } \\
\text { Model } 300 \text { CK }\end{array}$ & $\begin{array}{l}H-2-28883 \text { sht } 1 \\
H-2-28883 \text { sht } 2 \\
H-2-28888 \text { sht } 2\end{array}$ \\
\hline $\mathrm{CAH}-25-3$ & $\begin{array}{l}\text { Criticality Alarm Horn. Located in Building } \\
236-2 \text {, Floor } 5 \text {, Room } 50 \text {. }\end{array}$ & $\begin{array}{l}\text { Audible signaling Device: } \\
\text { Federal signal Corp. Model } \\
300 \text { GC, } 24 \text { VAC/DC Selectone } \\
\text { Connector Board: FS Corp. } \\
\text { Model } 300 \text { CK }\end{array}$ & $\begin{array}{l}H-2-28883 \text { sht } 1 \\
H-2-28883 \text { sht } 2 \\
H-2-28888 \text { sht } 2\end{array}$ \\
\hline $\mathrm{CAH}-25-4$ & $\begin{array}{l}\text { Criticality Alarm Horn. Located outside on } \\
\text { East Wall of Building } 236-2 \text {. }\end{array}$ & $\begin{array}{l}\text { Audible Signal ing Device: } \\
\text { Federal Signal Corp. Model } \\
300 \text { GC, } 24 \text { VAC/DC Selectone } \\
\text { Connector Board: FS Corp. } \\
\text { Model } 300 \text { CK }\end{array}$ & $\begin{array}{l}H-2-28883 \text { sht } 1 \\
H-2-28883 \text { sht } 2 \\
H-2-28888 \text { sht } 2\end{array}$ \\
\hline $\mathrm{CAH}-\mathrm{Z5}-5$ & $\begin{array}{l}\text { Criticality Alarm Horn. Located in Building } \\
236-2, \mathrm{Floor} 4 \text {, Room } 40 \text {. }\end{array}$ & $\begin{array}{l}\text { Audible Signaling Device: } \\
\text { Federal Signal corp. Model } \\
300 \text { GC, } 24 \text { VAC/DC Selectone } \\
\text { Connector Board: FS Corp. } \\
\text { Model } 300 \text { CK }\end{array}$ & $\begin{array}{l}H-2-28883 \text { sht } 1 \\
H-2-28883 \text { sht } 2 \\
H-2-28888 \text { sht } 2\end{array}$ \\
\hline CAH $-25-6$ & $\begin{array}{l}\text { Criticality Alarm Horn. Located in Building } \\
236-2 \text {, Floor 4, Room } 41 \text {. }\end{array}$ & $\begin{array}{l}\text { Audible Signaling Device: } \\
\text { Federal Signal Corp. Model } \\
300 \mathrm{GC}, 24 \text { VAC/DC Selectone } \\
\text { Connector Board: FS Corp. } \\
\text { Model } 300 \text { CK }\end{array}$ & $\begin{array}{l}H-2-28883 \text { sht } 1 \\
H-2-28883 \text { sht } 2 \\
H-2-28888 \text { sht } 2\end{array}$ \\
\hline
\end{tabular}




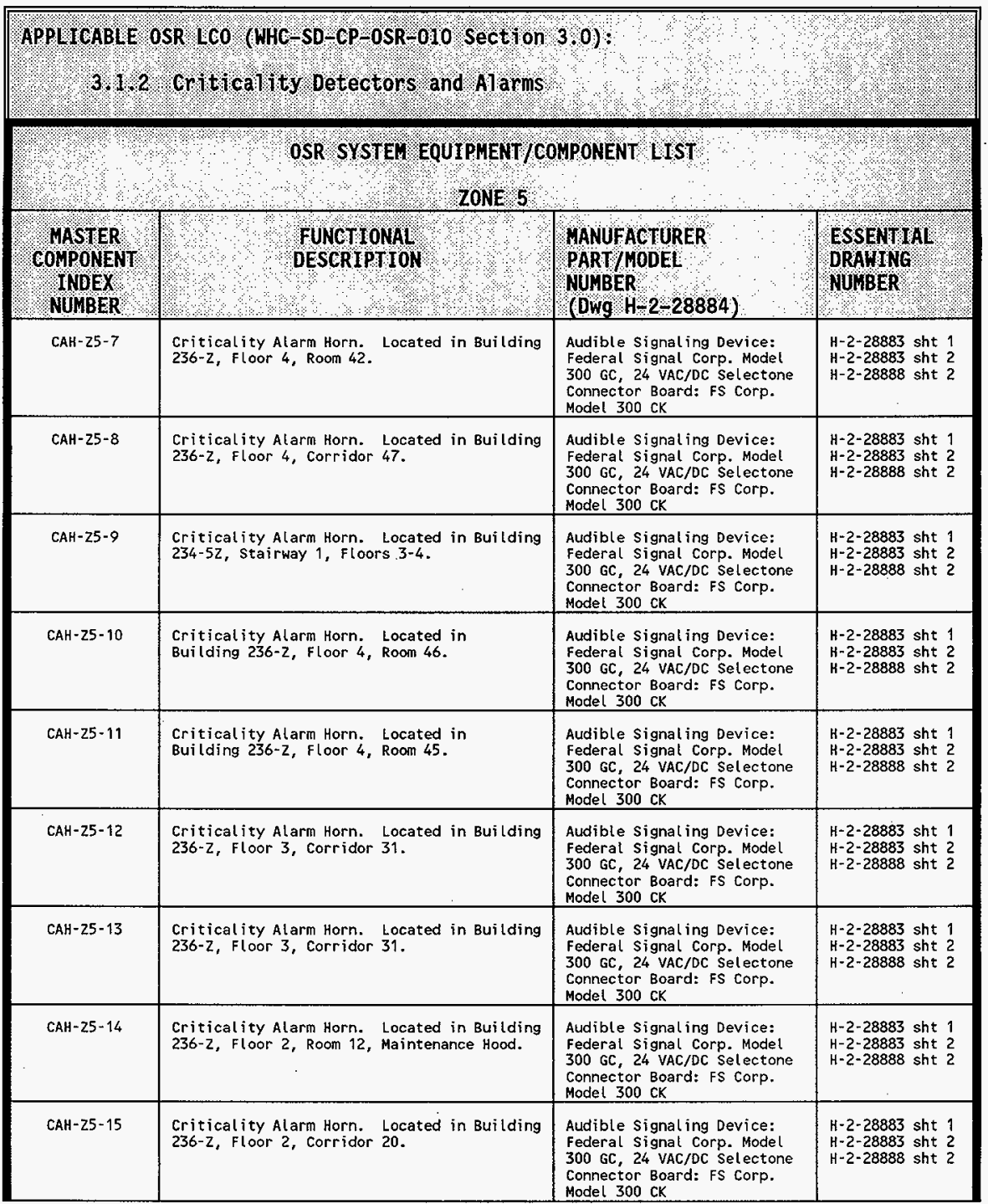




\begin{tabular}{|c|c|c|c|}
\hline \multirow{2}{*}{ APPLICABLC } & 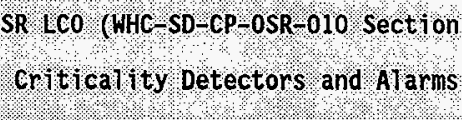 & $30):$ & $\therefore$ \\
\hline & 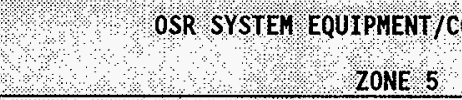 & PONENT LIST & \\
\hline $\begin{array}{l}\text { MASTER } \\
\text { COMPONENT } \\
\text { INDEX } \\
\text { NUNBER }\end{array}$ & 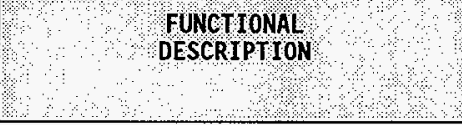 & $\begin{array}{l}\text { WANUFACTURER } \\
\text { PARTFHODEL } \\
\text { NUNBER } \\
\text { (DWG H } 2-28884 \text { ) }\end{array}$ & $\begin{array}{l}\text { ESSENTHA } \\
\text { BRAMTNG } \\
\text { UUMBER }\end{array}$ \\
\hline CAH $-25-17$ & $\begin{array}{l}\text { Criticality Alarm Horn. Located in Building } \\
236-2 \text {, Floor } 2 \text {, Corridor } 21 \text {. }\end{array}$ & $\begin{array}{l}\text { Audible signal ing Device: } \\
\text { Federal signal Corp. Model } \\
300 \text { GC, } 24 \text { VAC/DC Selectone } \\
\text { Connector Board: FS Corp. } \\
\text { Model 30D CK }\end{array}$ & $\begin{array}{l}H-2-28883 \text { sht } 1 \\
H-2-28883 \text { sht } 2 \\
H-2-28888 \text { sht } 2\end{array}$ \\
\hline CAH- $25-18$ & $\begin{array}{l}\text { Criticality Alarm Horn. Located in Building } \\
236-z \text {, Stairway } 2 \text {, Floor } 2 \text {. }\end{array}$ & $\begin{array}{l}\text { Audible Signal ing Device: } \\
\text { Federal Signal Corp. Model } \\
300 \mathrm{GC}, 24 \text { VAC/DC Selectone } \\
\text { Connector Board: FS Corp. } \\
\text { Model } 300 \mathrm{CK}\end{array}$ & $\begin{array}{l}H-2-28883 \text { sht } 1 \\
H-2-28883 \text { sht } 2 \\
H-2-28888 \text { sht } 2\end{array}$ \\
\hline $\mathrm{CAH}-25-19$ & $\begin{array}{l}\text { Criticality Alarm Horn. Located in Building } \\
236-2 \text {, Floor } 1 \text {, Corridor } 14 \text {. }\end{array}$ & $\begin{array}{l}\text { Audible signal ing Device: } \\
\text { Federal Signal Corp. Model } \\
300 \text { GC, } 24 \text { VAC/DC Selectone } \\
\text { Connector Board: FS Corp. } \\
\text { Model } 300 \text { CK }\end{array}$ & $\begin{array}{l}H-2-28883 \text { sht } 1 \\
H-2-28883 \text { sht } 2 \\
H-2-28888 \text { sht } 2\end{array}$ \\
\hline $\mathrm{CAH}-25-21$ & $\begin{array}{l}\text { Criticality Alarm Horn. Located in Building } \\
236-2 \text {, Floor } 1 \text {, Corridor } 11 .\end{array}$ & $\begin{array}{l}\text { Audible signal ing Device: } \\
\text { Federal signal Corp. Model } \\
300 \text { GC, } 24 \text { VAC/DC Selectone } \\
\text { Connector Board: FS Corp. } \\
\text { Model } 300 \text { CK }\end{array}$ & $\begin{array}{l}H-2-28883 \text { sht } 1 \\
H-2-28883 \text { sht } 2 \\
H-2-28888 \text { sht } 2\end{array}$ \\
\hline CALM-Z5-1 & $\begin{array}{l}\text { Critical ity Alarm Logic Module. Provides } \\
\text { the logic circuitry for annunciation of } \\
\text { alert and alarm conditions as detected by } \\
\text { the CADMs. Located in CAP- } 25 \text {. }\end{array}$ & Per Drawing H-2-91179 & $\begin{array}{l}H-2-28883 \text { sht } 1 \\
H-2-28883 \text { sht } 2 \\
H-2-28888 \text { sht } 2\end{array}$ \\
\hline CALM-25-2 & $\begin{array}{l}\text { Criticality Alarm Logic Module. Provides } \\
\text { the logic circuitry for annumciation of } \\
\text { alert and alarm conditions as detected by } \\
\text { the CADMs. Located in CAP- } 25 \text {. }\end{array}$ & Per Drawing $\mathrm{H}-2-91179$ & $\begin{array}{l}H-2-28883 \text { sht } 1 \\
H-2-28883 \text { sht } 2 \\
H-2-28888 \text { sht } 2\end{array}$ \\
\hline CALM-Z5-3 & $\begin{array}{l}\text { Critical ity Alarm Logic Module. Provides } \\
\text { the logic circuitry for annunciation of } \\
\text { alert and alarm conditions as detected by } \\
\text { the CADMs. Located in CAP- } 25 \text {. }\end{array}$ & Per Drawing $\mathrm{H}-2-91180$ & $\begin{array}{l}H-2-28883 \text { sht } 1 \\
H-2-28883 \text { sht } 2 \\
H-2-28888 \text { sht } 2\end{array}$ \\
\hline
\end{tabular}




\begin{tabular}{|c|c|c|c|}
\hline \multicolumn{4}{|c|}{ 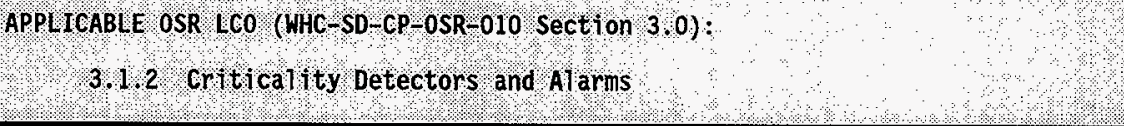 } \\
\hline+1 & OOSR SYSTEM EQUTPRENTIC & PONENT LST & \\
\hline $\begin{array}{l}\text { MASTER } \\
\text { COMPONENT } \\
\text { THDOX }\end{array}$ & FESCRTONAL PION & $\begin{array}{l}\text { WANUFACTURER } \\
\text { PART } \\
\text { WUWBER } \\
\text { (DWG }=2-28884)\end{array}$ & $\begin{array}{l}\text { ESSENTHE } \\
\text { DEAWING }\end{array}$ \\
\hline$C A P-25$ & $\begin{array}{l}\text { Criticality Alarm Panel Zone } 5 . \text { Contains } \\
\text { the CADMs, CALMs, CARM, Inhibit switches, } \\
\text { local (building) annunciation and Intertie } \\
\text { relays for the Zone } 5 \text { Criticality Alarm } \\
\text { System. Located in Building 236-z, Floor 4, } \\
\text { Corridor } 44 \text {. }\end{array}$ & $\begin{array}{l}\text { Relay Rack. } \\
\text { Premier, TVA } 7719-23\end{array}$ & $\begin{array}{l}H-2-28883 \text { sht } 1 \\
H-2-28883 \text { sht } 2 \\
H-2-28888 \text { sht } 2\end{array}$ \\
\hline CARM-Z5 & $\begin{array}{l}\text { Criticality Alarm Relay Module. Activates } \\
\text { the alarm signal generator and provides an } \\
\text { annunciation signal in the event of a high } \\
\text { alarm signal from the CALM. Includes an } \\
\text { inhibit switch to disable the alarm output } \\
\text { signal. Located in CAP-Z5. }\end{array}$ & Per Drawing H-2-91180 & $\begin{array}{l}H-2-28883 \text { sht } 1 \\
\text { H-2-28883 sht } 2 \\
\text { H-2-28888 sht } 2\end{array}$ \\
\hline $\begin{array}{l}\text { AC POWER RELAY } \\
\text { (Not required } \\
\text { for label ing } \\
\text { at this time.) }\end{array}$ & $\begin{array}{l}\text { (SC-3 SE) AC Power Monitor Relay. } \\
\text { Deenergizes on loss of power. A manual } \\
\text { reset is required to prevent a fluctuating } \\
\text { (erratic) power supply from initiating false } \\
\text { alarms. }\end{array}$ & Allen Bradley \# 700-C200-A1 & $\begin{array}{l}H-2-28883 \text { sht } 1 \\
H-2-28883 \text { sht } 2 \\
H-2-28888 \text { sht } 2\end{array}$ \\
\hline$S-5-1 A$ & $\begin{array}{l}\text { Inhibit Switch for CAD/CADM-25-1A used to } \\
\text { prevent annunciation of alarm during test, } \\
\text { cal ibration, and replacement of CADS and } \\
\text { CADMS. Located on CAP- } 25 \text {. }\end{array}$ & $\begin{array}{l}\text { Square D Class } 9001, \\
\text { Type LRSA-2K1 with } \\
\text { Type LA-1 Contact Blocks }\end{array}$ & $\begin{array}{l}H-2-28883 \text { sht } 1 \\
H-2-28883 \text { sht } 2 \\
H-2-28888 \text { sht } 2\end{array}$ \\
\hline$s-5-18$ & $\begin{array}{l}\text { Inhibit Switch for CAD/CADM-Z5-1B used to } \\
\text { prevent annunciation of alarm during test, } \\
\text { calibration, and replacement of CADS and } \\
\text { CADMs. Located on CAP- } 25 \text {. }\end{array}$ & $\begin{array}{l}\text { Square } D \text { Class } 9001, \\
\text { Type LRSA-2K1 with } \\
\text { Type LA-1 Contact Blocks }\end{array}$ & $\begin{array}{l}H-2-28883 \text { sht } 1 \\
H-2-28883 \text { sht } 2 \\
H-2-28888 \text { sht } 2\end{array}$ \\
\hline$s-5-1 c$ & $\begin{array}{l}\text { Inhibit Switch for CAD/CADM-25-1C used to } \\
\text { prevent annunciation of alarm during test, } \\
\text { cal ibration, and replacement of CADS and } \\
\text { CADMs. Located on CAP- } 25 \text {. }\end{array}$ & $\begin{array}{l}\text { Square D Class } 9001, \\
\text { Type LRSA-2K1 with } \\
\text { Type LA-1 Contact Blocks }\end{array}$ & $\begin{array}{l}H-2-28883 \text { sht } 1 \\
H-2-28883 \text { sht } 2 \\
H-2-28888 \text { sht } 2\end{array}$ \\
\hline
\end{tabular}




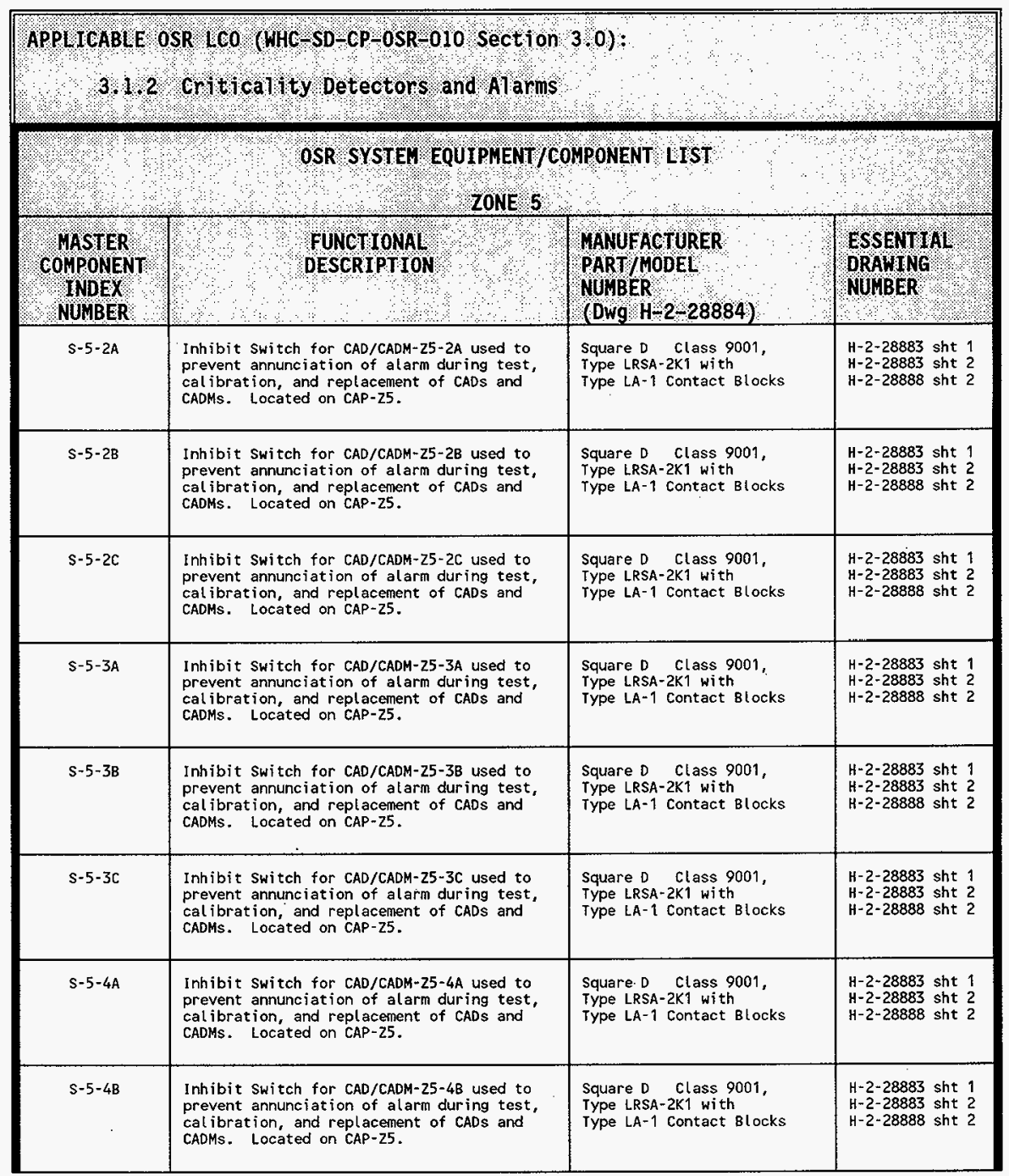




\begin{tabular}{|c|c|c|c|}
\hline \multicolumn{4}{|c|}{ 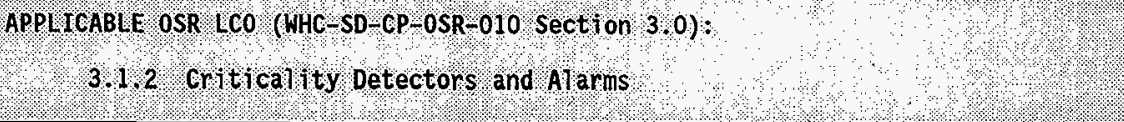 } \\
\hline $\begin{aligned} 4+3 \\
3\end{aligned}$ & 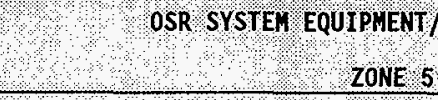 & IPONENT LIST & \\
\hline $\begin{array}{l}\text { WHSTER } \\
\text { COMPONENT } \\
\text { NWNDEX }\end{array}$ & PUNCTONAL DCRIPION & $\begin{array}{l}\text { MANUFACTURER } \\
\text { PART/MODEL } \\
\text { NUMBER } \\
\text { (DWG H } 2-28884)\end{array}$ & 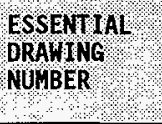 \\
\hline$s-5-4 c$ & $\begin{array}{l}\text { Inhibit Switch for CAD/CADM-Z5-4C used to } \\
\text { prevent annunciation of alarm during test, } \\
\text { calibration, and replacement of CADs and } \\
\text { CADMs. Located on CAP- } 25 \text {. }\end{array}$ & $\begin{array}{l}\text { Square D Class } 9001 \text {, } \\
\text { Type LRSA-2K1 with } \\
\text { Type LA-1 Contact Blocks }\end{array}$ & $\begin{array}{l}H-2-28883 \text { sht } 1 \\
H-2-28883 \text { sht } 2 \\
H-2-28888 \text { sht } 2\end{array}$ \\
\hline$s-5-5 A$ & $\begin{array}{l}\text { Inhibit Switch for CAD/CADM-25-5A used to } \\
\text { prevent annunciation of alarm during test, } \\
\text { calibration, and replacement of CADs and } \\
\text { CADMs. Located on CAP-25. }\end{array}$ & $\begin{array}{l}\text { Square } D \text { Class } 9001 \\
\text { Type LRSA-2K1 with } \\
\text { Type LA- } 1 \text { Contact Blocks }\end{array}$ & $\begin{array}{l}H-2-28883 \text { sht } 1 \\
H-2-28883 \text { sht } 2 \\
H-2-28888 \text { sht } 2\end{array}$ \\
\hline$s-5-5 B$ & $\begin{array}{l}\text { Inhibit Switch for CAD/CADM-25-5B used to } \\
\text { prevent annunciation of alarm during test, } \\
\text { calibration, and replacement of CADs and } \\
\text { CADMs. Located on CAP-Z5. }\end{array}$ & $\begin{array}{l}\text { Square D Class } 9001 \\
\text { Type LRSA-2K1 with } \\
\text { Type LA-1 Contact Blocks }\end{array}$ & $\begin{array}{l}H-2-28883 \text { sht } 1 \\
H-2-28883 \text { sht } 2 \\
H-2-28888 \text { sht } 2\end{array}$ \\
\hline$s-5-5 c$ & $\begin{array}{l}\text { Inhibit switch for CAD/CADM-Z5-5C used to } \\
\text { prevent annunciation of alarm during test, } \\
\text { cal ibration, and replacement of CADs and } \\
\text { CADMs. Located on CAP-25. }\end{array}$ & $\begin{array}{l}\text { Square } D \text { Class } 9001, \\
\text { Type LRSA-2K1 with } \\
\text { Type LA-1 Contact Blocks }\end{array}$ & $\begin{array}{l}H-2-28883 \text { sht } 1 \\
H-2-28883 \text { sht } 2 \\
H-2-28888 \text { sht } 2\end{array}$ \\
\hline$S-5-6 A$ & $\begin{array}{l}\text { Inhibit Switch for CAD/CADM- } 25-6 \mathrm{~A} \text { used to } \\
\text { prevent annunciation of alarm during test, } \\
\text { calibration, and replacement of CADS and } \\
\text { CADMs. Located on CAP-25. }\end{array}$ & $\begin{array}{l}\text { Square } 0 \text { Class } 9001 \text {, } \\
\text { Type LRSA-2K1 with } \\
\text { Type LA-1 Contact Blocks }\end{array}$ & $\begin{array}{l}H-2-28883 \text { sht } 1 \\
H-2-28883 \text { sht } 2 \\
H-2-28888 \text { sht } 2\end{array}$ \\
\hline$s-5-6 B$ & $\begin{array}{l}\text { Inhibit Switch for CAD/CADM- } 25-6 B \text { used to } \\
\text { prevent anhunciation of alarm during test, } \\
\text { cal ibration, and replacement of CADs and } \\
\text { CADMs. Located on CAP- } 25 \text {. }\end{array}$ & $\begin{array}{l}\text { Square } D \text { Class } 9001 \text {; } \\
\text { Type LRSA-2K1 with } \\
\text { Type LA-1 Contact Blocks }\end{array}$ & $\begin{array}{l}H-2-28883 \text { sht } 1 \\
H-2-28883 \text { sht } 2 \\
H-2-28888 \text { sht } 2\end{array}$ \\
\hline$s-5-6 c$ & $\begin{array}{l}\text { Inhibit Switch for CAD/CADM- } 25-6 C \text { used to } \\
\text { prevent annunciation of alarm during test, } \\
\text { cal ibration, and replacement of CADS and } \\
\text { CADMs. Located on CAP- } 25 \text {. }\end{array}$ & $\begin{array}{l}\text { Square D Class } 9001 \text {, } \\
\text { Type LRSA-2K1 with } \\
\text { Type LA-1 Contact Blocks }\end{array}$ & $\begin{array}{l}H-2-28883 \text { sht } 1 \\
H-2-28883 \text { sht } 2 \\
H-2-28888 \text { sht } 2\end{array}$ \\
\hline$s-5-7 A$ & $\begin{array}{l}\text { Inhibit Switch for CAD/CADM- } 25-7 A \text { used to } \\
\text { prevent annunciation of alarm during test, } \\
\text { calibration, and replacement of CADS and } \\
\text { CADMS. Located on CAP- } 25 \text {. }\end{array}$ & $\begin{array}{l}\text { Square D Class } 9001, \\
\text { Type LRSA-2K1 with } \\
\text { Type LA-1 Contact Blocks }\end{array}$ & $\begin{array}{l}\text { H-2-28883 sht } ? \\
\text { H-2-28883 sht } 2 \\
\text { H-2-28888 sht } 2\end{array}$ \\
\hline
\end{tabular}




\begin{tabular}{|c|c|c|c|}
\hline \multicolumn{4}{|c|}{ 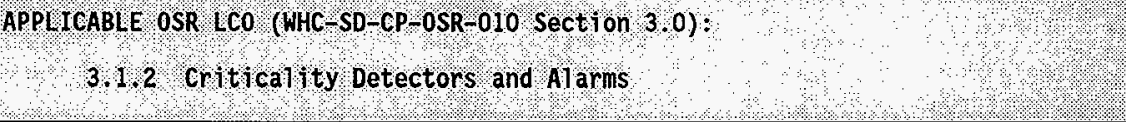 } \\
\hline & OSR SYSTEN EQUTPNENT & ONENT LIST & \\
\hline $\begin{array}{l}\text { MASTER } \\
\text { COMPONENT } \\
\text { TNDEX } \\
\text { NUNBER }\end{array}$ & FUNCT IONAL & $\begin{array}{l}\text { MANUFACTURER } \\
\text { PART/MODEL } \\
\text { NUHBER } \\
\text { (DWg } H-2-28884 \text { ) }\end{array}$ & $\begin{array}{l}\text { ESENTIL } \\
\text { DRAWING } \\
\text { NUMBER }\end{array}$ \\
\hline$s-5-78$ & $\begin{array}{l}\text { Inhibit Switch for CAD/CADM- } 25-78 \text { used to } \\
\text { prevent annunciation of alarm during test, } \\
\text { calibration, and replacement of CADS and } \\
\text { CADMs. Located on CAP-ZS. }\end{array}$ & $\begin{array}{l}\text { Square D Class } 9001 \text {, } \\
\text { Type LRSA-2K1 with } \\
\text { Type LA-1 Contact Blocks }\end{array}$ & $\begin{array}{l}H-2-28883 \text { sht } 1 \\
H-2-28883 \text { sht } 2 \\
H-2-28888 \text { sht } 2\end{array}$ \\
\hline$s-5-7 c$ & $\begin{array}{l}\text { Inhibit Switch for CAD/CADM- } 25-7 C \text { used to } \\
\text { prevent aninunciation of alarm during test, } \\
\text { calibration, and replacement of CADs and } \\
\text { CADMs. Located on CAP- } 25 \text {. }\end{array}$ & $\begin{array}{l}\text { Square D Class } 9001 \text {, } \\
\text { Type LRSA-2K1 with } \\
\text { Type LA-1 Contact Blocks }\end{array}$ & $\begin{array}{l}H-2-28883 \text { sht } 1 \\
H-2-28883 \text { sht } 2 \\
H-2-28888 \text { sht } 2\end{array}$ \\
\hline
\end{tabular}




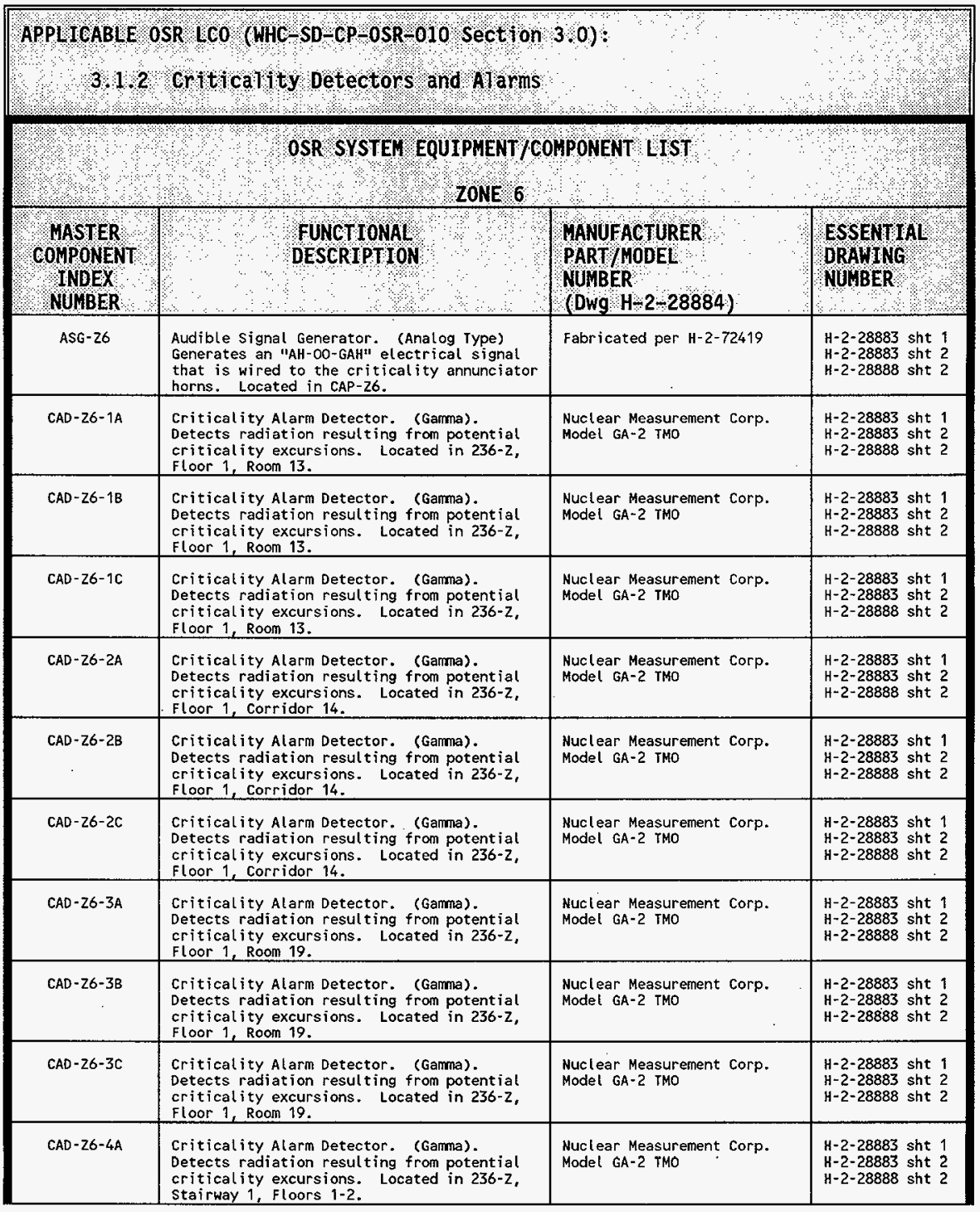




\begin{tabular}{|c|c|c|c|}
\hline 4 & 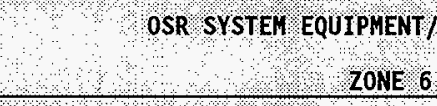 & ONENT LIST & \\
\hline $\begin{array}{l}\text { MASTER } \\
\text { QOMPONENT } \\
\text { NONDEX } \\
\text { NDMBER }\end{array}$ & ODESCRIPI ION & $\begin{array}{l}\text { MANUFACTURER } \\
\text { PART/MODEL } \\
\text { NUWBER } \\
\text { (DWg H } 2-28884)\end{array}$ & $\begin{array}{l}\text { CSSENATH } \\
\text { DRAWING } \\
\text { NUMBER }\end{array}$ \\
\hline$C A D-26-4 C$ & $\begin{array}{l}\text { Criticality Alarm Detector. (Gamma). } \\
\text { Detects radiation resulting from potential } \\
\text { criticality excursions. Located in } 236-2 \text {, } \\
\text { Stairway } 1 \text {, Floors } 1-2 \text {. }\end{array}$ & $\begin{array}{l}\text { Nuclear Measurement Corp. } \\
\text { Model GA-2 TMO }\end{array}$ & $\begin{array}{l}H-2-28883 \text { sht } 1 \\
H-2-28883 \text { sht } 2 \\
H-2-28888 \text { sht } 2\end{array}$ \\
\hline$C A D-26-5 A$ & $\begin{array}{l}\text { Criticality Alarm Detector. (Gamma). } \\
\text { Detects radiation resulting from potential } \\
\text { criticality excursions. Located in } 236-2 \text {, } \\
\text { Floor } 1 \text {, Corridor } 20 \text {. }\end{array}$ & $\begin{array}{l}\text { Nuclear Measurement Corp. } \\
\text { Model GA-2 TMO }\end{array}$ & $\begin{array}{l}H-2-28883 \text { sht } 1 \\
H-2-28883 \text { sht } 2 \\
H-2-28888 \text { sht } 2\end{array}$ \\
\hline$C A D-26-5 B$ & $\begin{array}{l}\text { Criticality Alarm Detector. (Gama). } \\
\text { Detects radiation resulting from potential } \\
\text { criticality excursions. Located in } 236-2 \text {, } \\
\text { Floor } 1 \text {, Corridor } 20 \text {. }\end{array}$ & $\begin{array}{l}\text { Nuclear Measurement Corp. } \\
\text { Model GA-2 TMO }\end{array}$ & $\begin{array}{l}H-2-28883 \text { sht } 1 \\
\text { H-2-28883 sht } 2 \\
\text { H-2-28888 sht } 2\end{array}$ \\
\hline$C A D-26-68$ & $\begin{array}{l}\text { Criticality Alarm Detector. (Garma). } \\
\text { Detects radiation resulting from potential } \\
\text { criticality excursions. Located in } 236-2 \text {, } \\
\text { Floorl, corridor } 21 \text {. }\end{array}$ & $\begin{array}{l}\text { Nuclear Measurement Corp. } \\
\text { Model GA-2 TMO }\end{array}$ & $\begin{array}{l}H-2-28883 \text { sht } 1 \\
\text { H-2-28883 sht } 2 \\
\text { H-2-28888 sht } 2\end{array}$ \\
\hline$C A D-26-6 C$ & $\begin{array}{l}\text { Criticality Alarm Detector. (Gamma). } \\
\text { Detects radiation resulting from potential } \\
\text { criticality excursions. Located in } 236-2 \text {, } \\
\text { Floor } 1 \text {, Corridor } 21 \text {. }\end{array}$ & $\begin{array}{l}\text { Nuclear Measurement Corp. } \\
\text { Model GA-2 TMO }\end{array}$ & $\begin{array}{l}H-2-28883 \text { sht } 1 \\
H-2-28883 \text { sht } 2 \\
H-2-28888 \text { sht } 2\end{array}$ \\
\hline$C A D-26-7 A$ & $\begin{array}{l}\text { Criticality Alarm Detector. (Gamma). } \\
\text { Detects radiation resulting from potential } \\
\text { criticality excursions. Located in } 236-2 \text {, } \\
\text { Stairway 2. Floors 2-3. }\end{array}$ & $\begin{array}{l}\text { Nuclear Measurement Corp. } \\
\text { Model GA-2 TMO }\end{array}$ & $\begin{array}{l}H-2-28883 \text { sht } 1 \\
H-2-28883 \text { sht } 2 \\
H-2-28888 \text { sht } 2\end{array}$ \\
\hline$C A D-Z 6-7 B$ & $\begin{array}{l}\text { Criticality Alarm Detector. (Gamma). } \\
\text { Detects radiation resulting from potential } \\
\text { criticality excursions. Located in } 236-2 \text {, } \\
\text { Stairway 2, Floors } 1-2 \text {. }\end{array}$ & $\begin{array}{l}\text { Nuclear Measurement Corp. } \\
\text { Model GA-2 TMO }\end{array}$ & $\begin{array}{l}H-2-28883 \text { sht } 1 \\
H+2-28883 \text { sht } 2 \\
H-2-28888 \text { sht } 2\end{array}$ \\
\hline$C A D-26-7 C$ & $\begin{array}{l}\text { Criticality Alarm Detector. (Gamma). } \\
\text { Detects radiation resulting from potential } \\
\text { criticality excursions. Located in } 236-2 \text {, } \\
\text { Stairway 2, Floors } 2-3 \text {. }\end{array}$ & $\begin{array}{l}\text { Nuclear Measurement Corp. } \\
\text { Model GA-2 TMO }\end{array}$ & $\begin{array}{l}H-2-28883 \text { sht } 1 \\
H-2-28883 \text { sht } 2 \\
H-2-28888 \text { sht } 2\end{array}$ \\
\hline
\end{tabular}




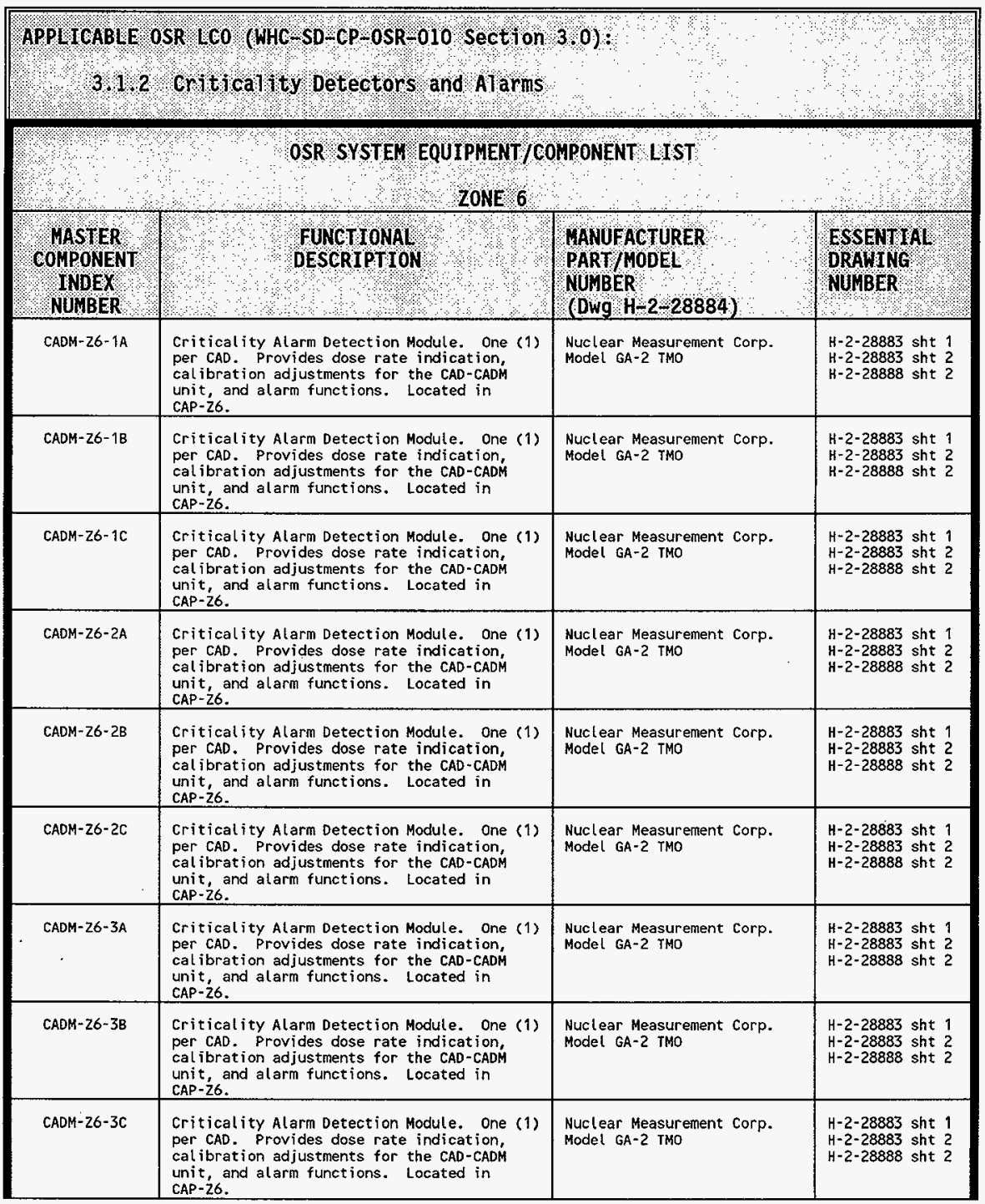




\begin{tabular}{|c|c|c|c|}
\hline \multicolumn{4}{|c|}{ 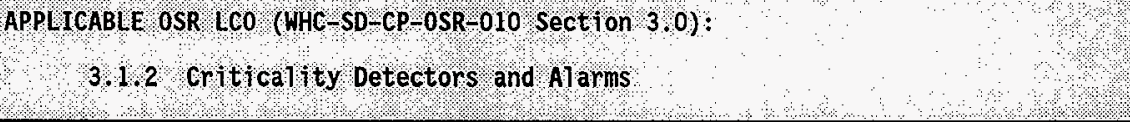 } \\
\hline 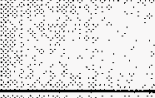 & OSR SYSTEMEQUIPNENT/C & PONENT IIST & \\
\hline $\begin{array}{l}\text { MASTER } \\
\text { GOMPONENT } \\
\text { NUNBER }\end{array}$ & 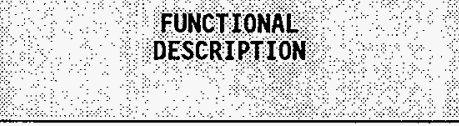 & $\begin{array}{l}\text { WANUFACTURER } \\
\text { PARTJYODEL } \\
\text { NUABER } \\
(\text { DWG }\end{array}$ & DSSENTHL \\
\hline$C A D M-26-4 B$ & $\begin{array}{l}\text { Criticality Alarm Detection Module. One (1) } \\
\text { per CAD. Provides dose rate indication, } \\
\text { catibration adjustments for the CAD-CADM } \\
\text { unit, and alarm functions. Located in } \\
\text { CAP-Z6. }\end{array}$ & $\begin{array}{l}\text { Nuclear Measurement Corp. } \\
\text { Model GA-2 TMO }\end{array}$ & $\begin{array}{l}H-2-28883 \text { sht } 1 \\
H-2-28883 \text { sht } 2 \\
H-2-28888 \text { sht } 2\end{array}$ \\
\hline$C A D M-26-4 C$ & $\begin{array}{l}\text { Criticality Alarm Detection Module. One (1) } \\
\text { per CAD. Provides dose rate indication, } \\
\text { calibration adjustments for the CAD-CADM } \\
\text { unit, and alarm functions. Located in } \\
\text { CAP-Z6. }\end{array}$ & $\begin{array}{l}\text { Nucl ear Measurement Corp. } \\
\text { Model GA-2 TMO }\end{array}$ & $\begin{array}{l}H-2-28883 \text { sht } 1 \\
H-2-28883 \text { sht } 2 \\
H-2-28888 \text { sht } 2\end{array}$ \\
\hline$C A D M-26-5 A$ & $\begin{array}{l}\text { Criticality Alarm Detection Module. One }(t) \\
\text { per CAD. Provides dose rate indication, } \\
\text { cal ibration adjustments for the CAD-CADM } \\
\text { unit, and alarm functions. Located in } \\
\text { CAP-Z6. }\end{array}$ & $\begin{array}{l}\text { Nuclear Measurement Corp. } \\
\text { Model GA-2 TMO }\end{array}$ & $\begin{array}{l}H-2-28883 \text { sht } 1 \\
H-2-28883 \text { sht } 2 \\
H-2-28888 \text { sht } 2\end{array}$ \\
\hline CADH-26-5C & $\begin{array}{l}\text { Criticality Alarm Detection Module. One (1) } \\
\text { per CAD. Provides dose rate indication, } \\
\text { cal ibration adjustments for the CAD-CADM } \\
\text { unit, and alarm functions. Located in } \\
\text { CAP-Z6. }\end{array}$ & $\begin{array}{l}\text { Nuclear Measurement Corp. } \\
\text { Hodel GA-2 TMO }\end{array}$ & $\begin{array}{l}H-2-28883 \text { sht } 1 \\
H-2-28883 \text { sht } 2 \\
H-2-28888 \text { sht } 2\end{array}$ \\
\hline CADM-Z6-6A & $\begin{array}{l}\text { Criticality Alarm Detection Hodule. One (1) } \\
\text { per CAD. Provides dose rate indication, } \\
\text { cal ibration adjustments for the CAD-CADM } \\
\text { unit, and alarm functions. Located in } \\
\text { CAP-Z6. }\end{array}$ & $\begin{array}{l}\text { Nuclear Measurement Corp. } \\
\text { Model GA-2 TMO }\end{array}$ & $\begin{array}{l}H-2-28883 \text { sht } 1 \\
H-2-28883 \text { sht } 2 \\
H-2-28888 \text { sht } 2\end{array}$ \\
\hline CADM- $Z 6-6 B$ & $\begin{array}{l}\text { Criticality Alarm Detection Module. One (1) } \\
\text { per CAD. Provides dose rate indication, } \\
\text { calibration adjustments for the CAD-CADM } \\
\text { unit, and alarm functions. Located in } \\
\text { CAP-z6. }\end{array}$ & $\begin{array}{l}\text { Nuclear Measurement Corp. } \\
\text { Model GA-2 THO }\end{array}$ & $\begin{array}{l}H-2-28883 \text { sht } 1 \\
H-2-28883 \text { sht } 2 \\
H-2-28888 \text { sht } 2\end{array}$ \\
\hline CADM-Z6-6C & $\begin{array}{l}\text { Criticality Alarm Detection Module. One (1) } \\
\text { per CAD. Provides dose rate indication, } \\
\text { cal ibration adjustments for the CAD-CADM } \\
\text { unit, and alarm functions. Located in } \\
\text { CAP-26. }\end{array}$ & $\begin{array}{l}\text { Nuclear Measurement Corp. } \\
\text { Model GA-2 TMO }\end{array}$ & $\begin{array}{l}H=2-28883 \text { sht } 1 \\
H-2-28883 \text { sht } 2 \\
H-2-28888 \text { sht } 2\end{array}$ \\
\hline
\end{tabular}




\begin{tabular}{|c|c|c|c|}
\hline $\begin{array}{l}\alpha \\
\because\end{array}$ & $\begin{array}{l}\text { OSR SYSTEN EQUIPNENT /C } \\
\text { 20NE } 6\end{array}$ & PONENT LIST & \\
\hline $\begin{array}{l}\text { MOSTER } \\
\text { COMPONENT } \\
\text { NUNDEX } \\
\end{array}$ & POUNCT IONAL & $\begin{array}{l}\text { PHANUFACTURER } \\
\text { PART/MODEL } \\
\text { WUMBER } \\
\text { (DWOH } 2-28884 \text { ) }\end{array}$ & $\begin{array}{l}\text { ESSEMTYM } \\
\text { DRAWHG } \\
\text { WUHBER }\end{array}$ \\
\hline$C A D M-26-78$ & $\begin{array}{l}\text { Criticality Alarm Detection Module. One (1) } \\
\text { per CAD. Provides dose rate indication, } \\
\text { calibration adjustments for the CAD-CADM } \\
\text { unit, and alarm functions. Located in } \\
\text { CAP-26. } 41\end{array}$ & $\begin{array}{l}\text { Nuclear Measurement Corp. } \\
\text { Model GA-2 TMO }\end{array}$ & $\begin{array}{l}H-2-28883 \text { sht } 1 \\
H-2-28883 \text { sht } 2 \\
H-2-28888 \text { sht } 2\end{array}$ \\
\hline CADM-Z6-7C & $\begin{array}{l}\text { Criticality Alarm Detection Module. One (1) } \\
\text { per CAD. Provides dose rate indication, } \\
\text { calibration adjustments for the CAD-CADM } \\
\text { unit, and alarm functions. Located in } \\
\text { CAP-Z6. }\end{array}$ & $\begin{array}{l}\text { Nuclear Measurement Corp. } \\
\text { Model GA-2 TMO }\end{array}$ & $\begin{array}{l}H-2-28883 \text { sht } 1 \\
H-2-28883 \text { sht } 2 \\
H-2-28888 \text { sht } 2\end{array}$ \\
\hline САH-Z6-1 & $\begin{array}{l}\text { Criticality Alarm Horn. Located in Building } \\
236-2 \text {, Floor 1, Room } 19 .\end{array}$ & $\begin{array}{l}\text { Audible signaling Device: } \\
\text { Federal signal Corp. Model } \\
300 \mathrm{GC}, 24 \text { VAC/DC Selectone } \\
\text { Connector Board: FS Corp. } \\
\text { Model } 300 \text { CK }\end{array}$ & $\begin{array}{l}H-2-28883 \text { sht } 1 \\
H-2-28883 \text { sht } 2 \\
H-2-28888 \text { sht } 2\end{array}$ \\
\hline CAH-Z6-3 & $\begin{array}{l}\text { Criticality Alarm Horn. Located in Building } \\
236-Z \text {, Floor 1, Room } 16 \text {. }\end{array}$ & $\begin{array}{l}\text { Audible signal ing Device: } \\
\text { Federal signal Corp. Model } \\
300 \text { GC, } 24 \text { VAC/DC Selectone } \\
\text { Connector Board: FS Corp. } \\
\text { Hodel } 300 \text { CK }\end{array}$ & $\begin{array}{l}H-2-28883 \text { sht } 1 \\
H-2-28883 \text { sht } 2 \\
H-2-28888 \text { sht } 2\end{array}$ \\
\hline CAH $-26-4$ & $\begin{array}{l}\text { Criticality Alarm Horn. Located in Building } \\
236-2 \text {, Floor 1, Corridor } 14 \text {. }\end{array}$ & $\begin{array}{l}\text { Audible Signaling Device: } \\
\text { Federal Signal Corp. Model } \\
300 \mathrm{GC}, 24 \text { VAC/DC Selectone } \\
\text { Connector Board: FS Corp. } \\
\text { Model } 300 \mathrm{CK}\end{array}$ & $\begin{array}{l}H-2-28883 \text { sht } 1 \\
H-2-28883 \text { sht } 2 \\
H-2-28888 \text { sht } 2\end{array}$ \\
\hline CAH-Z6-5 & $\begin{array}{l}\text { Criticality Alarm Horn. Located in Building } \\
236-2 \text {, Stairway } 3 \text {, floors } 1-2 \text {. }\end{array}$ & $\begin{array}{l}\text { Audible signaling Device: } \\
\text { Federal Signal Corp. Model } \\
300 \mathrm{GC}, 24 \text { vAC/DC Selectone } \\
\text { Connector Board: FS Corp. } \\
\text { Model } 300 \text { cK }\end{array}$ & $\begin{array}{l}H-2-28883 \\
H-2-28883 \\
\text { sht } 1 \\
H-2-28888 \\
\text { sht } 2\end{array}$ \\
\hline $\mathrm{CAH}-\mathrm{Z6}-6$ & $\begin{array}{l}\text { Criticality Alarm Horn. Located in Building } \\
236-z \text {, Floor 2, Room } 26 \text {. }\end{array}$ & $\begin{array}{l}\text { Audible signal ing Device: } \\
\text { Federal signal Corp. Model } \\
300 \mathrm{GC}, 24 \text { VAC/DC Selectone } \\
\text { Connector Board: FS Corp. } \\
\text { Model } 300 \mathrm{CK}\end{array}$ & $\begin{array}{l}H-2-28883 \text { sht } 1 \\
H-2-28883 \text { sht } 2 \\
H-2-28888 \text { sht } 2\end{array}$ \\
\hline
\end{tabular}




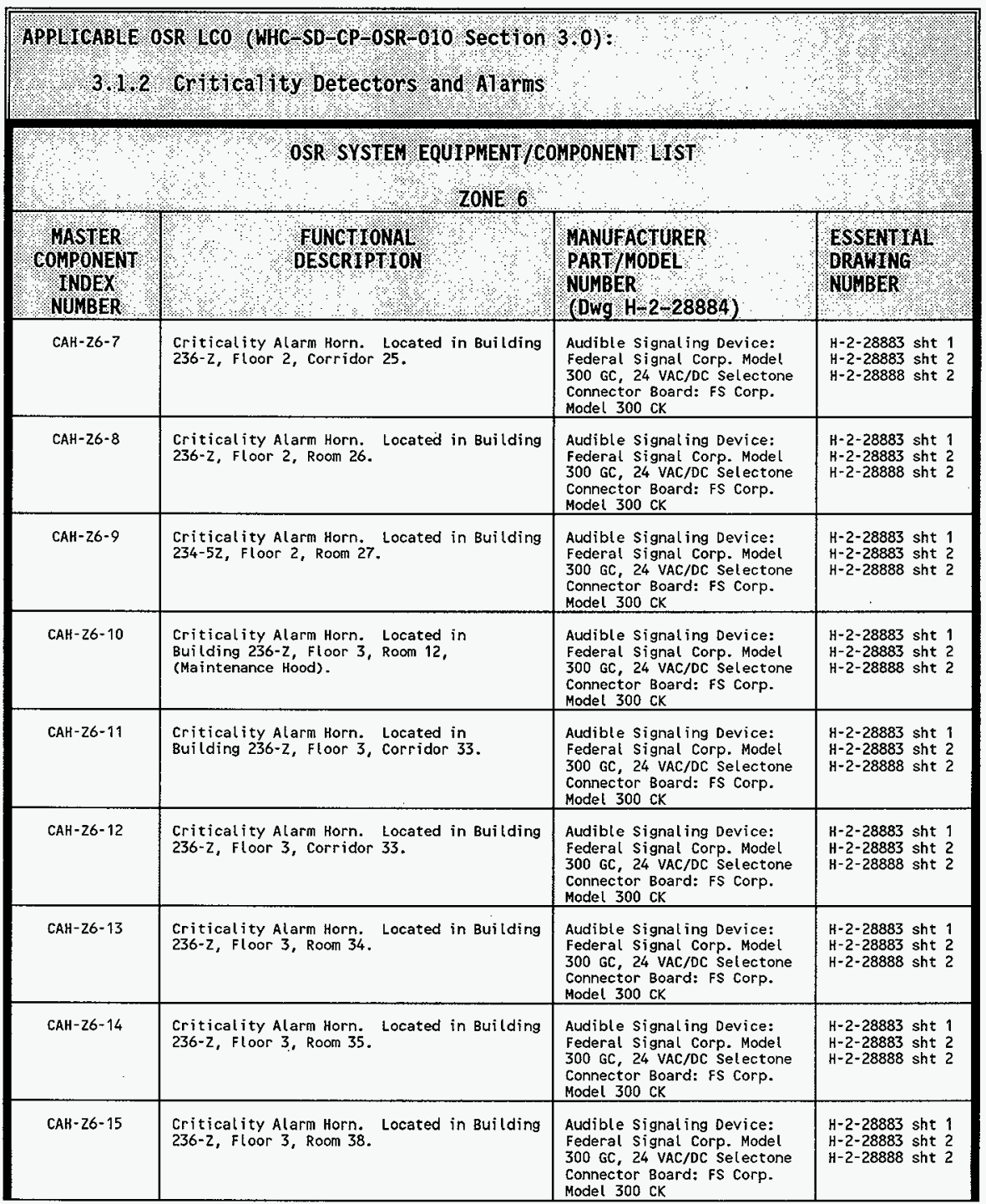




\begin{tabular}{|c|c|c|c|}
\hline $\begin{array}{ll} \\
1\end{array}$ & 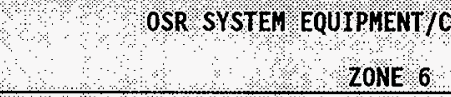 & PONENT LIST & \\
\hline $\begin{array}{l}\text { WASTER } \\
\text { COMPONENT } \\
\text { NONDEX } \\
\text { NBER }\end{array}$ & $\begin{array}{lll} & \\
& \end{array}$ & $\begin{array}{l}\text { MANUFACTURER } \\
\text { PARTPMODEL } \\
\text { WUNBER } \\
\text { (DWG H } 2-28884 \text { ) }\end{array}$ & 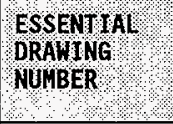 \\
\hline CAH-Z6-17 & $\begin{array}{l}\text { Criticality Alarm Horn. Located in Building } \\
236-2 \text {, Floor 4, Room } 43 \text {. }\end{array}$ & $\begin{array}{l}\text { Audible signaling Device: } \\
\text { Federal signal Corp. Model } \\
300 \mathrm{GC}, 24 \text { VAC/DC Selectone } \\
\text { Connector Board: FS Corp. } \\
\text { Model } 300 \text { CK }\end{array}$ & $\begin{array}{l}H-2-28883 \text { sht } 1 \\
H-2-28883 \text { sht } 2 \\
H-2-28888 \text { sht } 2\end{array}$ \\
\hline $\mathrm{CAH}-26-18$ & $\begin{array}{l}\text { Criticality Alarm Horn. Located in Building } \\
236-2 \text {, Stairway } 3 \text {, Floors } 3-4 \text {. }\end{array}$ & $\begin{array}{l}\text { Audible signaling Device: } \\
\text { Federal Signal Corp. Model } \\
300 \mathrm{GC}, 24 \text { VAC/DC Selectone } \\
\text { Connector Board: FS Corp. } \\
\text { Model } 300 \text { CK }\end{array}$ & $\begin{array}{l}H-2-28883 \text { sht } 1 \\
H-2-28883 \text { sht } 2 \\
H-2-28888 \text { sht } 2\end{array}$ \\
\hline CAH-Z6-19 & $\begin{array}{l}\text { Criticality Alarm Horn. Located in Building } \\
236-Z \text {, Floor 4, Room } 44 \text {. }\end{array}$ & $\begin{array}{l}\text { Audible signal ing Device: } \\
\text { Federal signal Corp. Model } \\
300 \text { GC, } 24 \text { VAC/OC Selectone } \\
\text { Connector Board: FS Corp. } \\
\text { Model } 300 \text { CK }\end{array}$ & $\begin{array}{l}H-2-28883 \text { sht } 1 \\
\text { H-2-28883 sht } 2 \\
H-2-28888 \text { sht } 2\end{array}$ \\
\hline $\mathrm{CAH}-\mathrm{Z6}-21$ & $\begin{array}{l}\text { Criticality Alarm Horn. Located in Building } \\
236-2 \text {, Floor 4, Room } 44 \text {. }\end{array}$ & $\begin{array}{l}\text { Audible signaling Device: } \\
\text { Federal Signal Corp. Model } \\
300 \text { GC, } 24 \text { VAC/DC Selectone } \\
\text { Connector Board: FS Corp. } \\
\text { Model } 300 \text { CK }\end{array}$ & $\begin{array}{l}H-2-28883 \text { sht } 1 \\
H-2-28883 \text { sht } 2 \\
H-2-28888 \text { sht } 2\end{array}$ \\
\hline CAH $-26-22$ & $\begin{array}{l}\text { Criticality Alarm Horn. Located in Building } \\
236-2 \text {, Floor 1, Room } 19 \text {. }\end{array}$ & $\begin{array}{l}\text { Audible Signaling Device: } \\
\text { Federal Signal Corp. Model } \\
300 \mathrm{GC}, 24 \text { VAC/DC Selectone } \\
\text { Connector Board: FS Corp. } \\
\text { Model } 300 \mathrm{CK}\end{array}$ & $\begin{array}{l}H-2-28883 \text { sht } 1 \\
H-2-28883 \text { sht } 2 \\
H-2-28888 \text { sht } 2\end{array}$ \\
\hline CALM-Z6-1 & $\begin{array}{l}\text { Criticality Alarm Logic Module. Provides } \\
\text { the logic circuitry for annunciation of } \\
\text { alert and alarm conditions as detected by } \\
\text { the cADMs. Located in CAP-26. }\end{array}$ & Per Drawing H-2-91179 & $\begin{array}{l}H-2-28883 \text { sht } 1 \\
H-2-28883 \text { sht } 2 \\
H-2-28888 \text { sht } 2\end{array}$ \\
\hline CALM-Z6-2 & $\begin{array}{l}\text { Criticality Alarm Logic Module. Provides } \\
\text { the logic circuitry for annunciation of } \\
\text { alert and alarm conditions as detected by } \\
\text { the CADHs. Located in CAP-Z6. }\end{array}$ & Per Drawing H-2-91179 & $\begin{array}{l}H-2-28883 \text { sht } 1 \\
H-2-28883 \text { sht } 2 \\
H-2-28888 \text { sht } 2\end{array}$ \\
\hline
\end{tabular}




\begin{tabular}{|c|c|c|c|}
\hline \multicolumn{4}{|c|}{ 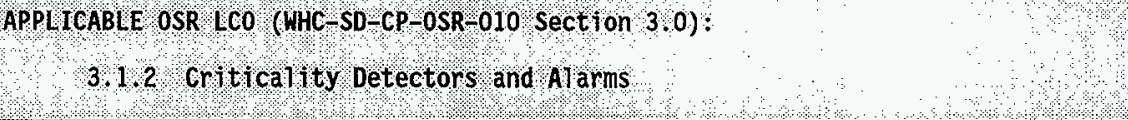 } \\
\hline $\begin{array}{cc}3 \\
2\end{array}$ & $\begin{array}{l}\text { OSR SYSTEM EQUIPHENT } 7 \mathrm{C} \\
\text { 20NE } 6\end{array}$ & PONENT LIST & \\
\hline $\begin{array}{l}\text { WMSIER } \\
\text { COMPONENT } \\
\text { NWDEK } \\
\text { NUMBER }\end{array}$ & 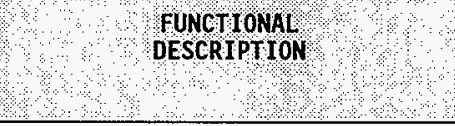 & $\begin{array}{l}\text { WANUFACTURER } \\
\text { PART/MODEL } \\
\text { NUNBER } \\
\text { (DWG } H-2-28884)\end{array}$ & $\begin{array}{l}\text { ESSENTHA } \\
\text { DRANHWG } \\
\text { NUMER }\end{array}$ \\
\hline CALM-26-4 & $\begin{array}{l}\text { Criticality Alarm Logic Module. Provides } \\
\text { the logic circuitry for annunciation of } \\
\text { alert and alarm conditions as detected by } \\
\text { the CADMs. Located in CAP-Z6. }\end{array}$ & Per Drawing $\mathrm{H}-2-91179$ & $\begin{array}{l}H-2-28883 \text { sht } 1 \\
H-2-28883 \text { sht } 2 \\
H-2-28888 \text { sht } 2\end{array}$ \\
\hline CALM-26-5 & $\begin{array}{l}\text { Criticality Alarm Logic Module. Provides } \\
\text { the logic circuitry for annunciation of } \\
\text { alert and alarm conditions as detected by } \\
\text { the CADMs. Located in CAP-Z6. }\end{array}$ & Per Drawing $\mathrm{H}-2-91179$ & $\begin{array}{l}H-2-28883 \text { sht } 1 \\
H-2-28883 \text { sht } 2 \\
H-2-28888 \text { sht } 2\end{array}$ \\
\hline CAP-26 & $\begin{array}{l}\text { Criticality Alarm Panel Zone } 6 \text {. Contains } \\
\text { the CADMs, CALMs, CARM, Inhibit switches, } \\
\text { local (building) annunciation and Intertie } \\
\text { relays for the Zone } 6 \text { Criticality Alarm } \\
\text { System. Located in Building } 236-2 \text { Corridor } \\
44 .\end{array}$ & $\begin{array}{l}\text { Relay Rack. } \\
\text { Premier, IVA } 7719-23\end{array}$ & $\begin{array}{l}H-2-28883 \text { sht } 1 \\
H-2-28883 \text { sht } 2 \\
H-2-28888 \text { sht } 2\end{array}$ \\
\hline CBS $-26-1$ & $\begin{array}{l}\text { Central Battery System. Nominal } 24 \text { VDC } \\
\text { system. Includes one } 12 \text { cell battery } \\
\text { capable of supplying power to the } \\
\text { criticality alarm system in excess of } 8 \\
\text { hours and charger capable of maintaining the } \\
\text { charge on the battery and while providing } \\
\text { normal power. Prinary supply power failure } \\
\text { annunciation. Located adjacent to CAP- } 25 \text {. }\end{array}$ & $\begin{array}{l}\text { 8attery: } \\
\text { Exide Power systems } 12 \text { or } \\
3 \text { CC-5 } \\
24 \text { VDC, } 100 \mathrm{~A} . \\
\text { Charger: } \\
\text { Exide Power systems UC } \\
26-25 \\
24 \text { VDC, } 25 \mathrm{~A}\end{array}$ & $\begin{array}{l}\text { H-2-28883 sht } 1 \\
\text { H-2-28883 sht } 2 \\
\text { H-2-28888 sht } 2\end{array}$ \\
\hline $\begin{array}{l}\text { AC POWER RELAY } \\
\text { (Not required } \\
\text { for labeling } \\
\text { at this time.) }\end{array}$ & $\begin{array}{l}\text { (SC-3 SE) AC Power Monitor Relay. } \\
\text { Deenergizes on loss of power. A manual } \\
\text { reset is required to prevent a fluctuating } \\
\text { (erratic) power supply from initiating false } \\
\text { alarms. }\end{array}$ & Allen Bradley \# 700-C200-Al & $\begin{array}{l}\text { H-2-28883 sht } 1 \\
\text { H-2-28883 sht. } 2 \\
\text { H-2-28888 sht } 2\end{array}$ \\
\hline$s-6-1 A$ & $\begin{array}{l}\text { Inhibit Switch for CAD/CADM-Z6-1A used to } \\
\text { prevent annunciat ion of alarm during test, } \\
\text { calibration, and replacement of CADS and } \\
\text { CADMs. Located on CAP-Z6. }\end{array}$ & $\begin{array}{l}\text { Square D Class } 9001 \text {, } \\
\text { Type LRSA-2K1 with } \\
\text { Type LA-1 Contact Blocks }\end{array}$ & $\begin{array}{l}\text { H-2-28883 sht } 1 \\
\text { H-2-28883 sht } 2 \\
\text { H-2-28888 sht } 2\end{array}$ \\
\hline
\end{tabular}




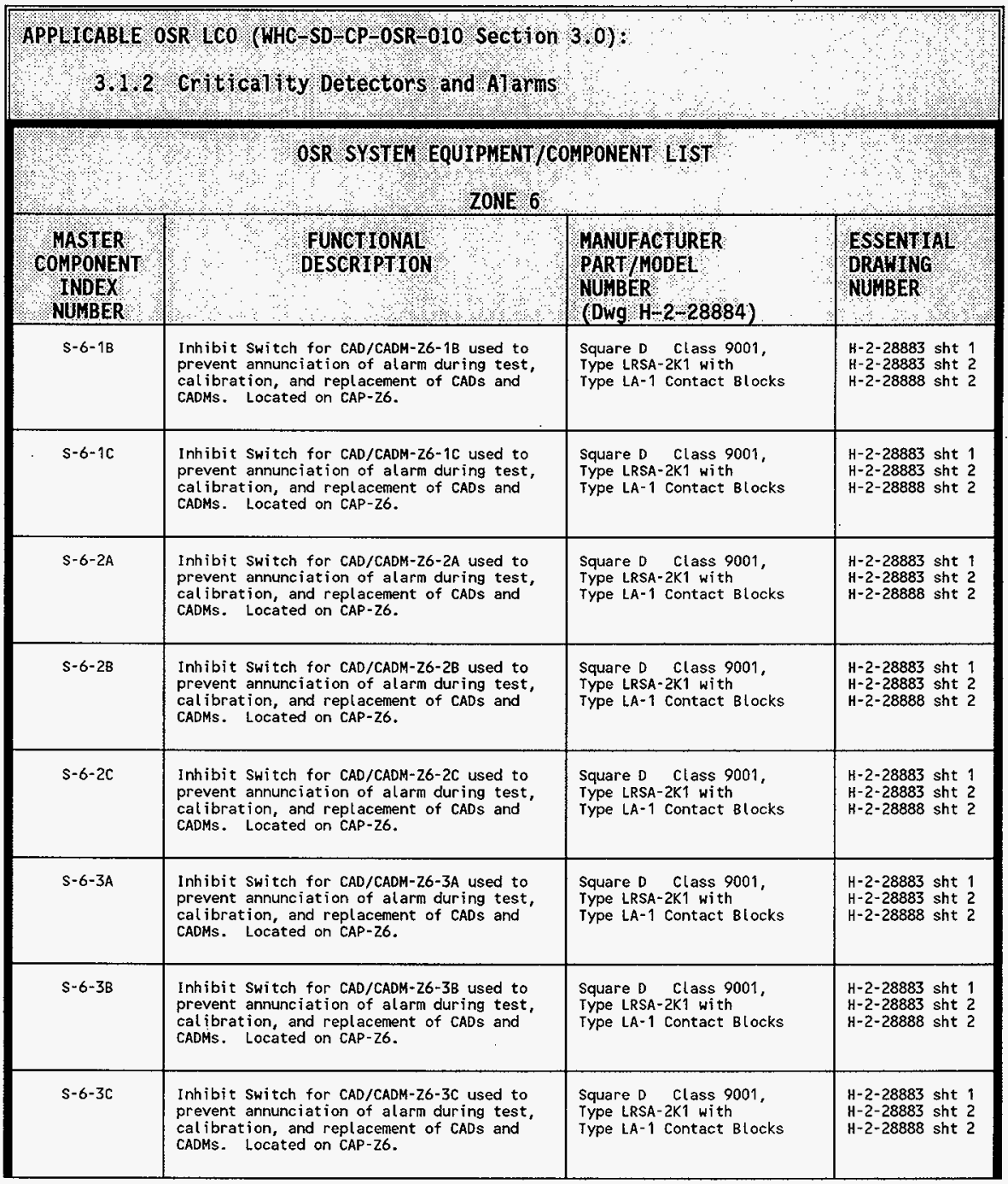




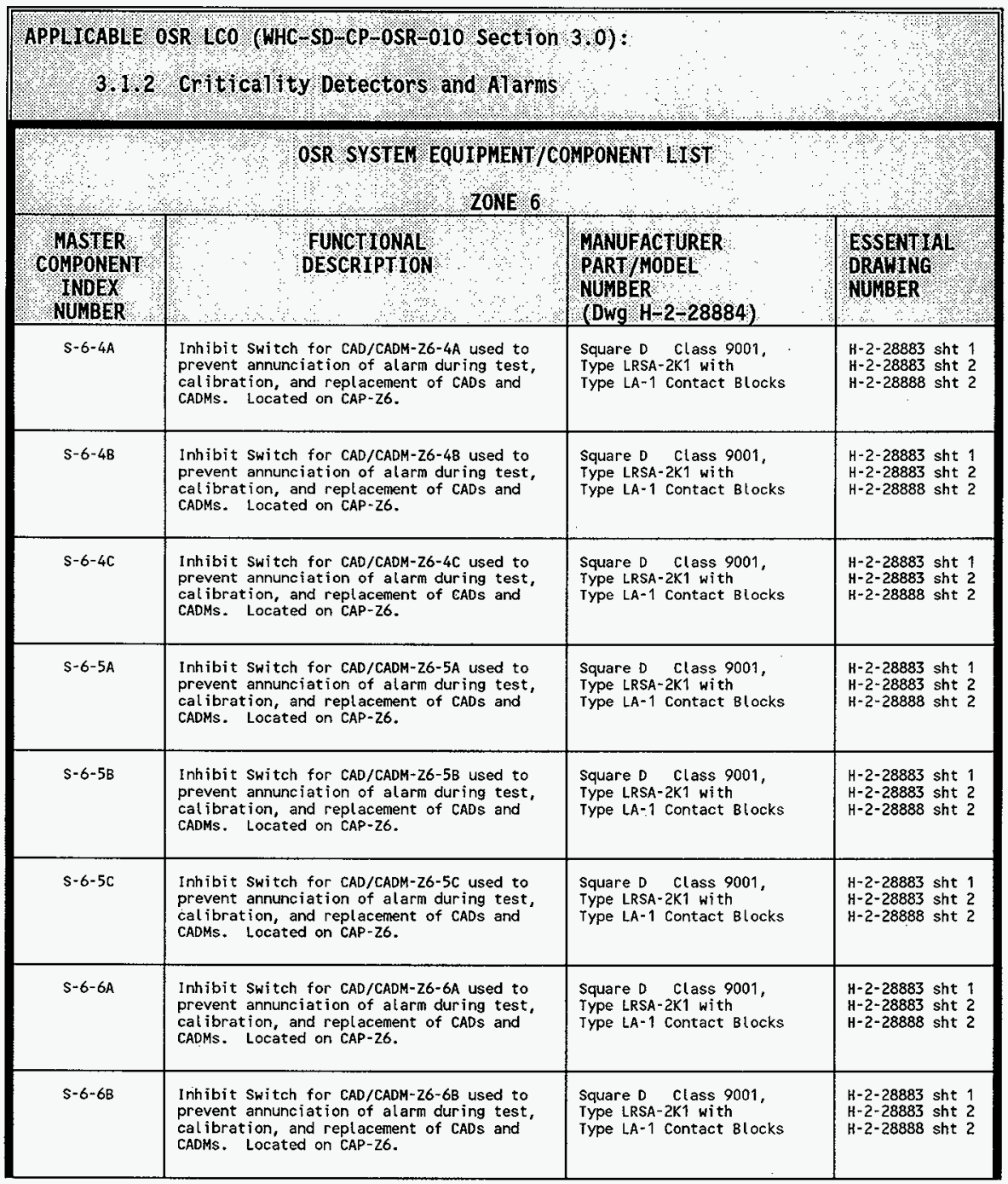




\begin{tabular}{|c|c|c|c|}
\hline \multicolumn{4}{|c|}{ APPLICABLE OSR $\angle C O$ (WHC SD CP-OSR-010 section 3,0$)$} \\
\hline & 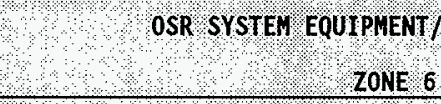 & MPONENT LIST & \\
\hline $\begin{array}{l}\text { YASTER } \\
\text { COMPONENT } \\
\text { INDEX } \\
\text { NUMBER }\end{array}$ & $\begin{array}{l}\text { FUNCTIONAL } \\
\text { DESCRIPTION }\end{array}$ & $\begin{array}{l}\text { NANUFACTURER } \\
\text { PART THODEL } \\
\text { NUMBER } \\
\text { (DWg H-2 } 28884 \text { ) }\end{array}$ & $\begin{array}{l}\text { ESSENTIAL } \\
\text { DRAHING } \\
\text { NUMBER }\end{array}$ \\
\hline$s-6-6 c$ & $\begin{array}{l}\text { Inhibit Switch for CAD/CADM-26-6C used to } \\
\text { prevent annunciation of alarm during test, } \\
\text { calibration, and replacement of CADS and } \\
\text { CADMs. Located on CAP-26. }\end{array}$ & $\begin{array}{l}\text { Square D Class } 9001, \\
\text { Type LRSA-2K1 with } \\
\text { Type LA-1 Contact Blocks }\end{array}$ & $\begin{array}{l}y-2-28883 \text { sht } 1 \\
H-2-28883 \text { sht } 2 \\
H-2-28888 \text { sht } 2\end{array}$ \\
\hline$s-6-7 A$ & $\begin{array}{l}\text { Inhibit Switch for CAD/CADM-Z6-7A used to } \\
\text { prevent annunciation of alarm during test, } \\
\text { calibration, and replacement of CADS and } \\
\text { CADMS. Located on CAP-Z6. }\end{array}$ & $\begin{array}{l}\text { Square D Class } 9001 \text {, } \\
\text { Type LRSA-2K1 with } \\
\text { Type LA-1 Contact Blocks }\end{array}$ & $\begin{array}{l}H-2-28883 \text { sht } 1 \\
H-2-28883 \text { sht } 2 \\
H-2-28888 \text { sht } 2\end{array}$ \\
\hline$s-6-78$ & $\begin{array}{l}\text { Inhibit. Switch for CAD/CADM-26-7B used to } \\
\text { prevent annunciation of alarm during test, } \\
\text { calibration, and replacement of CADS and } \\
\text { CADMs. Located on CAP-26. }\end{array}$ & $\begin{array}{l}\text { Square D Class } 9001 \text {, } \\
\text { Type LRSA-2K1 with } \\
\text { Type LA-1 Contact Blocks }\end{array}$ & $\begin{array}{l}\text { H-2-28883 sht } 1 \\
H-2-28883 \text { sht } 2 \\
H-2-28888 \text { sht } 2\end{array}$ \\
\hline$s-6-7 c$ & $\begin{array}{l}\text { Inhibit Switch for CAD/CADM-26-7C used to } \\
\text { prevent annunciation of alarm during test, } \\
\text { calibration, and replacement of CADS and } \\
\text { CADMs. Located on CAP-26. }\end{array}$ & $\begin{array}{l}\text { Square D Class } 9001 \text {, } \\
\text { Type LRSA-2K1 with } \\
\text { Type LA-1 Contact Blocks }\end{array}$ & $\begin{array}{l}H-2-28883 \text { sht } 1 \\
H-2-28883 \text { sht } 2 \\
H-2-28888 \text { sht } 2\end{array}$ \\
\hline
\end{tabular}




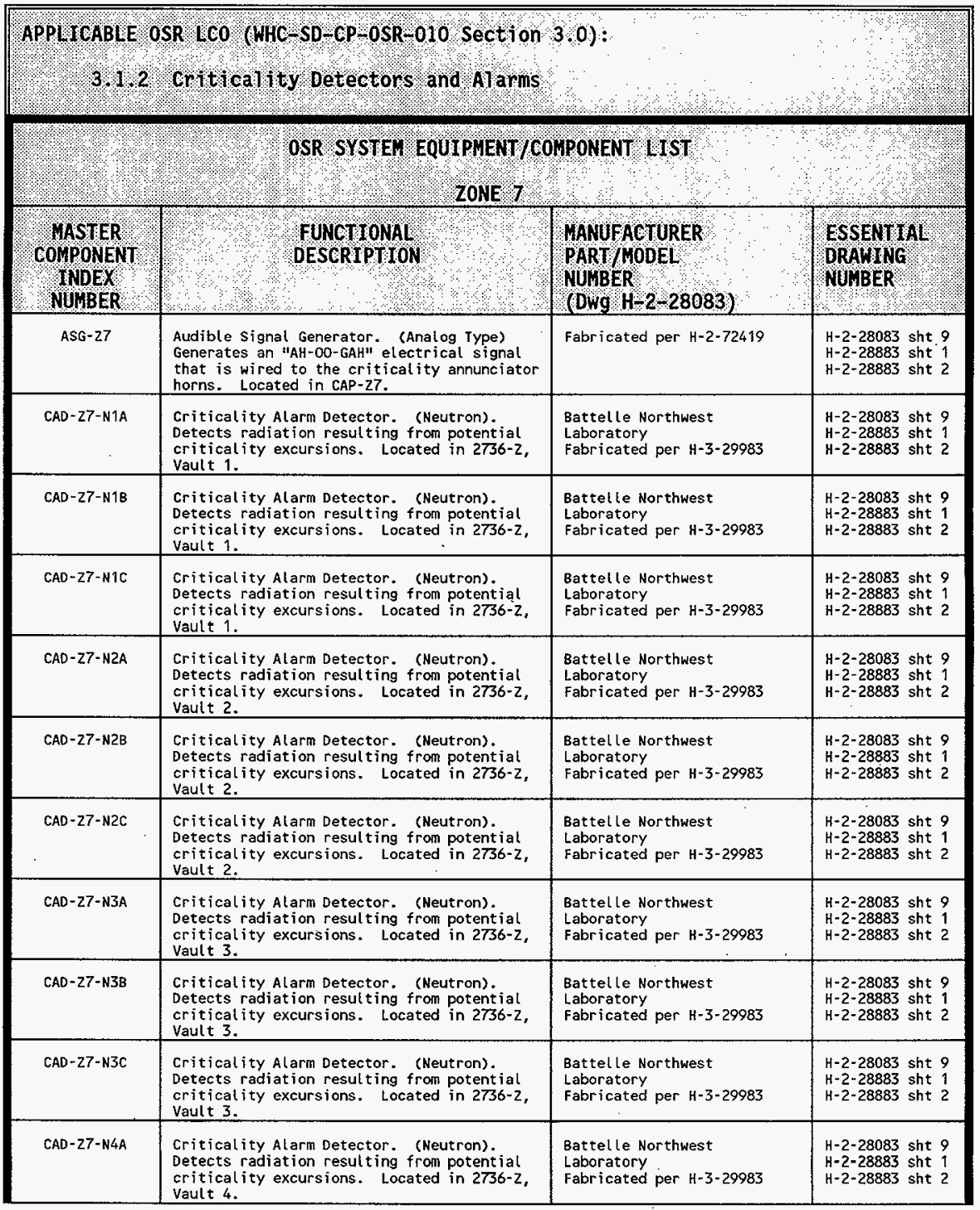




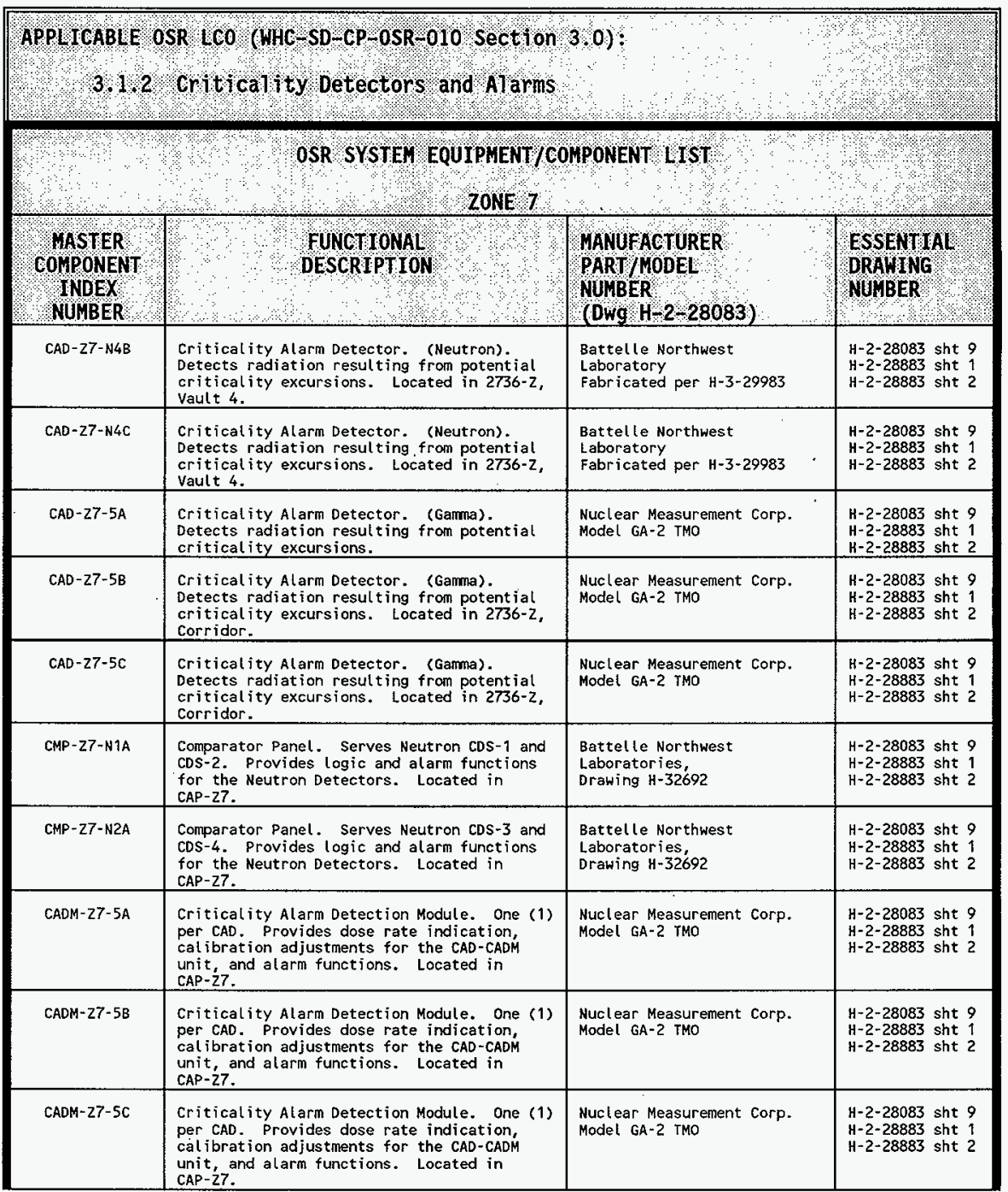




\begin{tabular}{|c|c|c|c|}
\hline \multicolumn{4}{|c|}{ 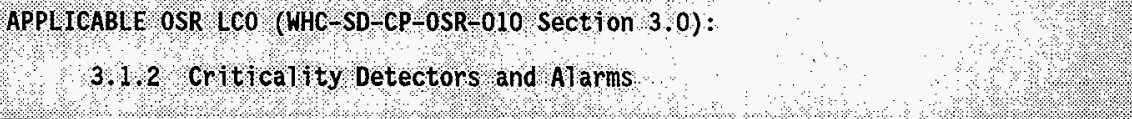 } \\
\hline & OSR SYSTEY EQUIPNENT/ & ONENT LIST & \\
\hline $\begin{array}{l}\text { WASTER } \\
\text { COMPONENT } \\
\text { NUDEX } \\
\end{array}$ & $\begin{array}{l}\text { PONCTIONAL } \\
\text { DESCRIPTION }\end{array}$ & $\begin{array}{l}\text { MANUFACTURER } \\
\text { PART/ MODEL } \\
\text { MUMBER } \\
\text { (DWG H } 2-28083 \text { ) }\end{array}$ & $\begin{array}{l}\text { ESSE WHAL } \\
\text { DRANGNG } \\
\text { NUMBER }\end{array}$ \\
\hline CAF - 1 & $\begin{array}{l}\text { Critical ity Alarm Flasher. Blue light used } \\
\text { to suppl ement audible criticality alarms in } \\
\text { areas with high background sound. Located in } \\
2736-Z A \text {, North Room. }\end{array}$ & North American ST-1250/Blue & $\begin{array}{l}H-2-28083 \text { sht } 9 \\
H-2-28883 \text { sht } 1 \\
H-2-28883 \text { sht } 2\end{array}$ \\
\hline CAF-2 & $\begin{array}{l}\text { Criticality Alarm flasher. Blue light used } \\
\text { to supplement audible criticality alarms in } \\
\text { areas with high background sound. Located in } \\
\text { 2736-ZA, North Room. }\end{array}$ & North American ST-1250/Blue & $\begin{array}{l}\text { H-2-2-28083 sht } 9 \\
\text { H-2-28883 sht } 1 \\
\text { H-2-28883 sht } 2\end{array}$ \\
\hline CAF -3 & $\begin{array}{l}\text { Criticality Alarm Flasher. Blue light used } \\
\text { to supplement audible criticatity alarms in } \\
\text { areas with high background sound. Located in } \\
2736-Z A \text {, North Room. }\end{array}$ & North American ST-1250/Blue & $\begin{array}{l}H-2-28083 \text { sht } 9 \\
H-2-28883 \text { sht } 1 \\
H-2-28883 \text { sht } 2\end{array}$ \\
\hline CAF -4 & $\begin{array}{l}\text { Criticality Alarm Flasher. Blue light used } \\
\text { to supplement audible criticality alarms in } \\
\text { areas with high background sound. Located in } \\
\text { 2736-ZA, South Room. }\end{array}$ & North American ST-1250/Blue & $\begin{array}{l}\text { H-2-28083 sht } 9 \\
\text { H-2-28883 sht } 1 \\
\text { H-2-28883 sht } 2\end{array}$ \\
\hline $\mathrm{CAH}-27-1$ & $\begin{array}{l}\text { Criticality Alarm Horn. Located in Vault } 1 \\
\text { of } 2736-z \text {. }\end{array}$ & $\begin{array}{l}\text { Audible signaling Device: } \\
\text { Federal signal Corp. Model } \\
300 \mathrm{GC}, 24 \text { VAC/DC Selectone } \\
\text { Connector Board: FS Corp. } \\
\text { Model } 300 \mathrm{CK}\end{array}$ & $\begin{array}{l}H-2-28083 \text { sht } 9 \\
H-2-28883 \text { sht } 1 \\
H-2-28883 \text { sht } 2\end{array}$ \\
\hline $\mathrm{CAH}-27-2$ & $\begin{array}{l}\text { Criticality Alarm Horn. Located in Vault } 1 \\
\text { of } 2736-2 \text {. }\end{array}$ & $\begin{array}{l}\text { Audible signaling Device: } \\
\text { Federal Signal Corp. Model } \\
300 \mathrm{GC}, 24 \text { VAC/DC Selectone } \\
\text { Connector Board: FS Corp. } \\
\text { Model } 300 \mathrm{CK}\end{array}$ & $\begin{array}{l}\text { H-2-28083 sht } 9 \\
\text { H-2-28883 sht } 1 \\
\text { H-2-28883 sht } 2\end{array}$ \\
\hline САH $-27-3$ & $\begin{array}{l}\text { Criticality Alarm Horn. Located in Vault } 2 \\
\text { of } 2736-2 \text {. }\end{array}$ & $\begin{array}{l}\text { Audible Signal ing Device: } \\
\text { Federal signal Corp. Model } \\
300 \text { GC, } 24 \text { VAC/DC Selectone } \\
\text { Connector } 80 a r d: \text { FS Corp. } \\
\text { Model } 300 \mathrm{CK} \\
\end{array}$ & $\begin{array}{l}\text { H-2-28083 sht } 9 \\
\text { H-2-28883 sht } 1 \\
\text { H-2-28883 sht } 2\end{array}$ \\
\hline $\mathrm{CAH}-\mathrm{Z7}-4$ & $\begin{array}{l}\text { Criticality Alarm Horn. Located in vault } 2 \\
\text { of } 2736-2 \text {. }\end{array}$ & $\begin{array}{l}\text { Audible Signaling Device: } \\
\text { Federal signal Corp. Model } \\
300 \mathrm{GC}, 24 \text { VAC/DC Selectone } \\
\text { Connector Board: FS Corp. } \\
\text { Model } 300 \mathrm{CK}\end{array}$ & $\begin{array}{l}\text { H-2-28083 sht } 9 \\
\text { H-2-28883 sht } 1 \\
\text { H-2-28883 sht } 2\end{array}$ \\
\hline CAH-Z7-5 & $\begin{array}{l}\text { Criticality Alarm Horn. Located in Vault } 3 \\
\text { of } 2736-2 \text {. }\end{array}$ & $\begin{array}{l}\text { Audible Signaling Device: } \\
\text { Federal signal Corp. Model } \\
300 \mathrm{GC}, 24 \text { VAC/DC Selectone } \\
\text { Connector Board: FS Corp. } \\
\text { Model } 300 \text { CK }\end{array}$ & $\begin{array}{l}\text { H-2-28083 sht } 9 \\
\text { H-2-28883 sht } 1 \\
\text { H-2-28883 sht } 2\end{array}$ \\
\hline
\end{tabular}




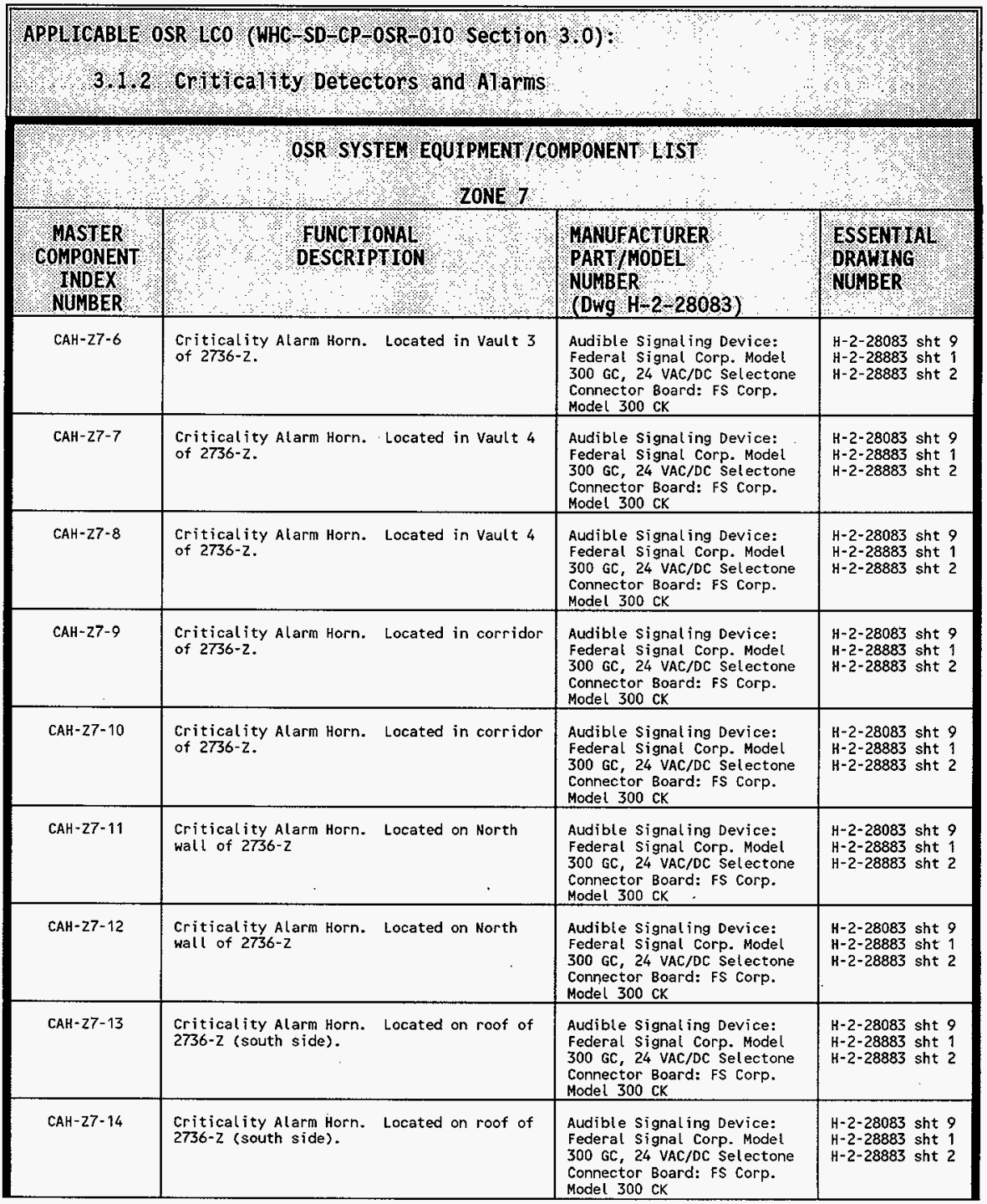




\begin{tabular}{|c|c|c|c|}
\hline \multicolumn{4}{|c|}{ 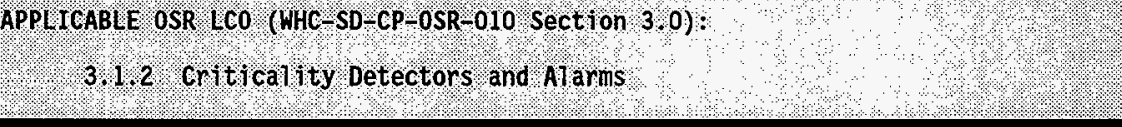 } \\
\hline (1) & $\begin{array}{l}\text { OSR SYSTEN EQUIPHENT } \\
\\
\end{array}$ & & \\
\hline $\begin{array}{l}\text { WASTER } \\
\text { COMPONENT } \\
\text { MWDEX } \\
\text { NUMBER }\end{array}$ & FONCTONAL DCRTPION & $\begin{array}{l}\text { WANUFACTURER } \\
\text { PART/MODEL } \\
\text { NUWBER } \\
\text { (DWG } \mathrm{H}-2-28083 \text { ) }\end{array}$ & $\begin{array}{l}\text { ESSENTIL } \\
\text { ORAWING } \\
\text { NUNBER }\end{array}$ \\
\hline $\mathrm{CAH}-27-16$ & Criticality Alarm Horn. Located in 2736-zA. & $\begin{array}{l}\text { Audible signaling Device: } \\
\text { Federal Signal Corp. Model } \\
300 \text { GC, } 24 \text { VAC/DC Selectone } \\
\text { Connector Board: FS Corp. } \\
\text { Model } 300 \text { CK }\end{array}$ & $\begin{array}{l}H-2-28083 \text { sht } 9 \\
H-2-28883 \text { sht } 1 \\
H-2-28883 \text { sht } 2\end{array}$ \\
\hline CALM- $27-1$ & $\begin{array}{l}\text { Criticality Alarm Logic Module. Provides } \\
\text { the logic circuitry for annunciation of } \\
\text { alert and alarm conditions as detected by } \\
\text { the CADMs. }\end{array}$ & Per Drawing $\mathrm{H}-2-91179$ & $\begin{array}{l}H-2-28083 \text { sht } 9 \\
H-2-28883 \text { sht } ? \\
H-2-28883 \text { sht } 2\end{array}$ \\
\hline CAP -27 & $\begin{array}{l}\text { Criticality Alarm Panel Zone } 7 \text {. Contains } \\
\text { the Neutron and Gamma Detection system } \\
\text { associated components including comparator } \\
\text { panels, CADMs, CALM, CARM, inh ibit switches, } \\
\text { system interconnect ion, local (building) } \\
\text { annunciation, and Intertie relays for the } \\
\text { Zone } 7 \text { Criticality Alarm System. Located in } \\
\text { Building 2736-2A. }\end{array}$ & Purchase Spec \# IED2736CA1 & $\begin{array}{l}H-2-28083 \text { sht } 9 \\
H-2-28883 \text { sht } 1 \\
H-2-28883 \text { sht } 2\end{array}$ \\
\hline CBS-Z7 & $\begin{array}{l}\text { Central Battery system. Nominal } 24 \text { VDC } \\
\text { system. Includes one } 12 \text { cell battery } \\
\text { capable of supplying power to the CAHs, } \\
\text { CAFs, and Gamma detection system associated } \\
\text { with CAP-z7 in excess of } 8 \text { hours and charger } \\
\text { capable of maintaining the charge on the } \\
\text { battery and while providing normal power. } \\
\text { Primary supply power failure annunciation. } \\
\text { Located adjacent to CAP- } 27 \text {. }\end{array}$ & Purchase Spec \# IED2736CA1 & $\begin{array}{l}H-2-28083 \text { sht } 9 \\
H-2-28883 \text { sht } 1 \\
H-2-28883 \text { sht } 2\end{array}$ \\
\hline $\begin{array}{l}\text { PLS- } 77-1 \\
\text { (Not required } \\
\text { for labeling } \\
\text { at this time.) }\end{array}$ & $\begin{array}{l}\text { (NON-SE). LOSs of Power Annunciators. } \\
\text { Located in Corridor of } 2736-2 \text { (near Vault } 1 \\
\text { door). }\end{array}$ & $\begin{array}{l}\text { Federal Model } 450,24 \text { VDC } \\
\text { Vibratone Horn }\end{array}$ & $\begin{array}{l}H-2-28083 \text { sht } 9 \\
H-2-28883 \text { sht } 1 \\
H-2-28883 \operatorname{sht} 2\end{array}$ \\
\hline $\begin{array}{l}\text { PLS }-27-2 \\
\text { (Not required } \\
\text { for labeling } \\
\text { at this time.) }\end{array}$ & $\begin{array}{l}\text { (NON-SE). Loss of Power Annunciators. } \\
\text { Located in Corridor of } 2736-2 \text { (near Vaut } 1 \\
\text { door). }\end{array}$ & $\begin{array}{l}\text { Federal Model } 350,120 \text { VAC, } \\
60 \text { Hertz Vibratone Horn }\end{array}$ & $\begin{array}{l}H-2-28083 \text { sht } 9 \\
H-2-28883 \text { sht } 1 \\
H-2-28883 \text { sht } 2\end{array}$ \\
\hline
\end{tabular}




\begin{tabular}{|c|c|c|c|}
\hline \multicolumn{4}{|c|}{ 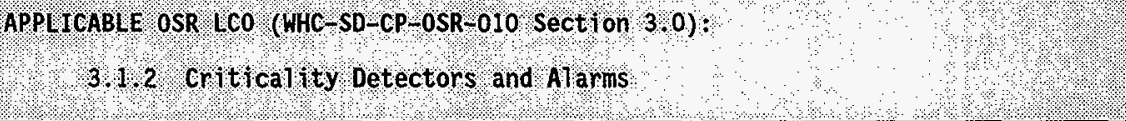 } \\
\hline${ }^{\prime} \quad \mathrm{s}$ & $\begin{array}{l}\text { OSR SYSTEM EQUIPHENT/ } \\
\text { ZONE } 7\end{array}$ & PONENT LIST & \\
\hline $\begin{array}{l}\text { MASTER } \\
\text { QOMPONENT } \\
\text { THDEX } \\
\text { WUMBER }\end{array}$ & $\begin{array}{l}\text { EUNCTIONAL } \\
\text { DESCRIPION }\end{array}$ & $\begin{array}{l}\text { HANUFACTURER } \\
\text { PART/MODEL } \\
\text { NUMBER } \\
\text { (DWGH-2-28083) }\end{array}$ & $\begin{array}{l}\text { ESSENA } \\
\text { DRAMHG } \\
\text { NUNBER }\end{array}$ \\
\hline $\begin{array}{l}\text { AC POWER RELAY } \\
\text { (Not required } \\
\text { for label ing } \\
\text { at this time.) }\end{array}$ & $\begin{array}{l}\text { (SC-3 SE). AC Power Monitor Relay. } \\
\text { Deenergizes on loss of power. A manual } \\
\text { reset is required to prevent a fluctuating } \\
\text { (erratic) power supply from initiating a } \\
\text { false alarm. }\end{array}$ & Allen Bradley \# C700-200-A1 & $\begin{array}{l}\text { H-2-28083 sht } 9 \\
H-2-28883 \text { sht } 1 \\
H-2-28883 \text { sht } 2\end{array}$ \\
\hline$s-7-5 A$ & $\begin{array}{l}\text { Inhibit switch for CAD/CADM- } 7 Z-5 A \text { used to } \\
\text { prevent annunciat ion of alarm during test, } \\
\text { cal ibration, and replacement of CADs and } \\
\text { CADMs. Located on CAP-Z7. }\end{array}$ & $\begin{array}{l}\text { Square D Class } 9001, \\
\text { Type LRSA-2K1 with } \\
\text { Type LA-1 Contact Blocks }\end{array}$ & $\begin{array}{l}H-2-28083 \text { sht } 9 \\
H-2-28883 \text { sht } 1 \\
\dot{H}-2-28883 \text { sht } 2\end{array}$ \\
\hline$s-7-5 B$ & $\begin{array}{l}\text { Inhibit Switch for CAD/CADM-7Z-5B used to } \\
\text { prevent annunciat ion of alarm during test, } \\
\text { cal ibration, and replacement of CADS and } \\
\text { CADMs. Located on CAP- } 27 \text {. }\end{array}$ & $\begin{array}{l}\text { Square D Class } 9001, \\
\text { Type LRSA-2K1 with } \\
\text { Type LA-1 Contact Blocks }\end{array}$ & $\begin{array}{l}H-2-28083 \text { sht } 9 \\
H-2-28883 \text { sht } 1 \\
H-2-28883 \text { sht } 2\end{array}$ \\
\hline$s-7-5 c$ & $\begin{array}{l}\text { Inhibit Switch for CAD/CADH- } 72-5 C \text { used to } \\
\text { prevent annunciation of alarm during test, } \\
\text { cal ibration, and replacement of CADs and } \\
\text { CADMs. Located on CAP- } 27 \text {. }\end{array}$ & $\begin{array}{l}\text { Square } D \text { Class } 9001, \\
\text { Type LRSA-2K1 with } \\
\text { Type LA- } 1 \text { Contact Blocks }\end{array}$ & $\begin{array}{l}H-2-28083 \text { sht } 9 \\
H-2-28883 \text { sht } 1 \\
H-2-28883 \text { sht } 2\end{array}$ \\
\hline UPS- 27 & $\begin{array}{l}\text { Uninterruptible Power Supply. Provides } \\
\text { interrupted power to Neutron Critical ity } \\
\text { Detection System. }\end{array}$ & Purchase Spec \# IED2736CA1 & $\begin{array}{l}\text { H-2-28083 sht } 9 \\
\text { H-2-28883 sht } 1 \\
\text { H-2-28883 sht } 2\end{array}$ \\
\hline
\end{tabular}




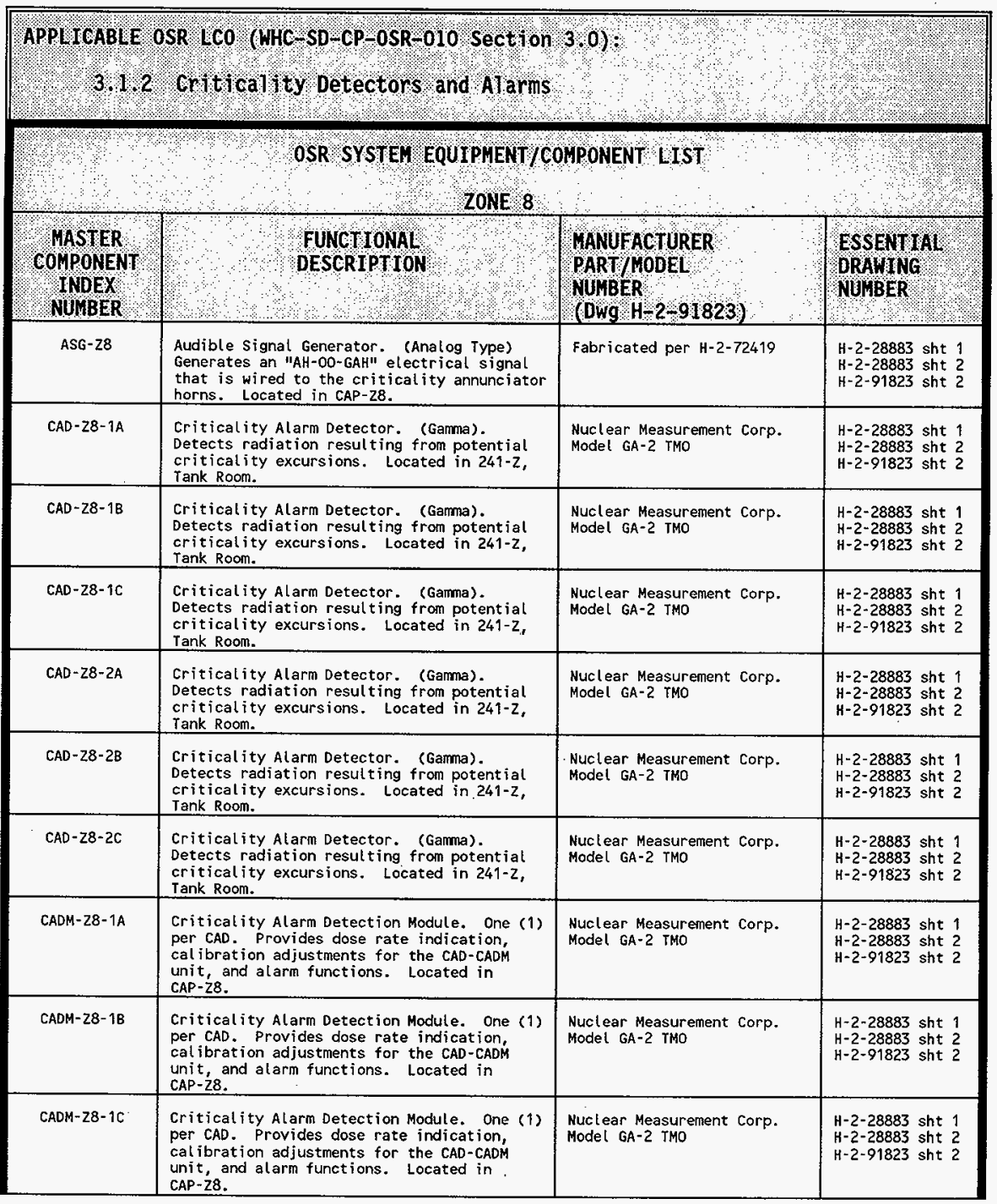




\begin{tabular}{|c|c|c|c|}
\hline \multicolumn{4}{|c|}{ 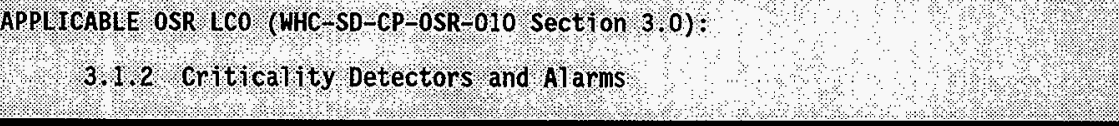 } \\
\hline +. & 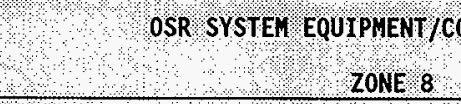 & PONENT LIST & \\
\hline $\begin{array}{l}\text { WASTER } \\
\text { COMPONENT } \\
\text { TNDEX } \\
\text { NUMBER }\end{array}$ & PENCTIONAL DESCRIPION & $\begin{array}{l}\text { WANUFACTURER } \\
\text { PART/HODEL } \\
\text { NUNBER } \\
\text { (DWg H-2-91823) }\end{array}$ & $\begin{array}{l}\text { ESSENTHL } \\
\text { DRAMHA } \\
\text { NGWBER }\end{array}$ \\
\hline CADM-Z8-2B & $\begin{array}{l}\text { Criticality Alarm Detection Module. One (1) } \\
\text { per CAD. Provides dose rate indication, } \\
\text { calibration adjustments for the CAD-CADM } \\
\text { unit, and alarm functions. Located in } \\
\text { CAP-Z8. }\end{array}$ & $\begin{array}{l}\text { Nuclear Measurement Corp. } \\
\text { Model GA-2 TMO }\end{array}$ & $\begin{array}{l}H-2-28883 \text { sht } 1 \\
H-2-28883 \text { sht } 2 \\
H-2-91823 \text { sht } 2\end{array}$ \\
\hline CADM-Z8-2C & $\begin{array}{l}\text { Criticality Alarm Detection Module. One (1) } \\
\text { per CAD. Provides dose rate indication, } \\
\text { calibration adjustments for the CAD-CADM } \\
\text { unit, and alarm functions. Located in } \\
\text { CAP- } 28 \text {. }\end{array}$ & $\begin{array}{l}\text { Nuclear Measurement Corp. } \\
\text { Model GA-2 TMO }\end{array}$ & $\begin{array}{l}H-2-28883 \text { sht } 1 \\
H-2-28883 \text { sht } 2 \\
H-2-91823 \text { sht } 2\end{array}$ \\
\hline $\mathrm{CAH}-\mathrm{ZB}-1$ & $\begin{array}{l}\text { Criticality Alarm horn. Located outside on } \\
\text { West Wall of Building } 241-z \text {. }\end{array}$ & $\begin{array}{l}\text { Audible signal ing Device: } \\
\text { Federal signal Corp. Model } \\
300 \text { GC, } 24 \text { VAC/DC Selectone } \\
\text { Connector Board: FS Corp. } \\
\text { Model } 300 \text { CK }\end{array}$ & 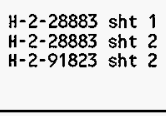 \\
\hline $\mathrm{CAH}-28-3$ & $\begin{array}{l}\text { Criticality Alarm Horn. Located in Building } \\
241-2 \text { Tank Room, West wall. }\end{array}$ & $\begin{array}{l}\text { Audible signal ing Device: } \\
\text { Federal Signal Corp. Model } \\
300 \mathrm{GC}, 24 \text { VAC/DC Selectone } \\
\text { Connector Board: FS Corp. } \\
\text { Model } 300 \text { CK }\end{array}$ & $\begin{array}{l}H-2-28883 \text { sht } 1 \\
H-2-28883 \text { sht } 2 \\
H-2-91823 \text { sht } 2\end{array}$ \\
\hline $\mathrm{CAH}-\mathrm{ZB}-4$ & $\begin{array}{l}\text { Criticality Alarm Horn. Located in Building } \\
241-2 \text { Tank Room, West Wall. }\end{array}$ & $\begin{array}{l}\text { Audible signal ing Device: } \\
\text { Federal signal Corp. Model } \\
300 \text { GC, } 24 \text { VAC/DC Selectone } \\
\text { Connector Board: FS Corp. } \\
\text { Model } 300 \text { CK }\end{array}$ & $\begin{array}{l}H-2-28883 \text { sht } 1 \\
H-2-28883 \text { sht } 2 \\
H-2-91823 \text { sht } 2\end{array}$ \\
\hline$C A H-28-5$ & $\begin{array}{l}\text { Criticality Alarm Horn. Located in Building } \\
241-2 \text { Tank Room, East Wall. }\end{array}$ & $\begin{array}{l}\text { Audible Signal ing Device: } \\
\text { Federal Signal Corp. Model } \\
300 \mathrm{GC}, 24 \text { VAC/DC Selectone } \\
\text { Connector Board: FS Corp. } \\
\text { Model } 300 \text { CK }\end{array}$ & $\begin{array}{l}H-2-28883 \text { sht } 1 \\
H-2-28883 \text { sht } 2 \\
H-2-91823 \text { sht } 2\end{array}$ \\
\hline $\mathrm{CAH}-28-6$ & $\begin{array}{l}\text { Criticality Alarm Horn. Located in Building } \\
241-2 \text { Tank Room, East Wall. }\end{array}$ & $\begin{array}{l}\text { Audible Signaling Device: } \\
\text { Federal Signal Corp. Model } \\
300 \mathrm{GC}, 24 \text { VAC/DC Selectone } \\
\text { Connector Board: FS Corp. } \\
\text { Model } 300 \text { CK }\end{array}$ & $\begin{array}{l}H-2-28883 \text { sht } 1 \\
H-2-28883 \text { sht } 2 \\
H-2-91823 \text { sht } 2\end{array}$ \\
\hline
\end{tabular}




\begin{tabular}{|c|c|c|c|}
\hline \multicolumn{4}{|c|}{ 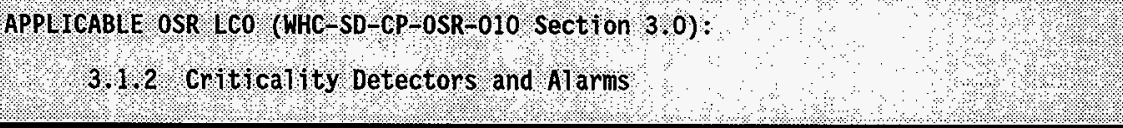 } \\
\hline 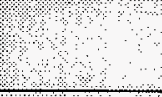 & $\begin{array}{l}\text { OSR SYSTEN EQUIPHENTIC } \\
\text { YONE } 8\end{array}$ & PONENT LIST & \\
\hline $\begin{array}{l}\text { TASTER } \\
\text { COMPONENT } \\
\text { TNDEX } \\
\text { NUHBER }\end{array}$ & PUNCTIONAL & $\begin{array}{l}\text { MANUFACTURER } \\
\text { PARTJWODEL } \\
\text { NUWBER } \\
\text { (DWg } H=2,91823)\end{array}$ & 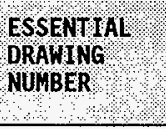 \\
\hline CAH- $28-8$ & $\begin{array}{l}\text { Criticality Alarm Horn. Located outside on } \\
\text { East Wall of Building } 241-2 \text {. }\end{array}$ & $\begin{array}{l}\text { Audible Signal ing Device: } \\
\text { Federal Signal Corp. Model } \\
300 \mathrm{GC}, 24 \text { VAC/DC Selectone } \\
\text { Connector Board: FS Corp. } \\
\text { Model } 300 \mathrm{CK}\end{array}$ & $\begin{array}{l}H-2-28883 \text { sht } 1 \\
H=2-28883 \text { sht } 2 \\
H-2-91823 \text { sht } 2\end{array}$ \\
\hline CALM-28-1 & $\begin{array}{l}\text { Criticality Alarm Logic Module. Provides } \\
\text { the logic circuitry for annunciation of } \\
\text { alert and alarm conditions as detected by } \\
\text { the CADMs. Located in CAP-28. }\end{array}$ & Per Drawing $H-2-91179$ & $\begin{array}{l}H-2-28883 \text { sht } 1 \\
H-2-28883 \text { sht } 2 \\
H-2-91823 \text { sht } 2\end{array}$ \\
\hline$C A P-28$ & $\begin{array}{l}\text { Criticality Alarm Panel Zone } 8 \text {. Contains } \\
\text { the CADMs, CALMS, CARM, Inhibit switches, } \\
\text { and local (building) annunciation relays for } \\
\text { the Zone } 8 \text { Criticality Alarm System. } \\
\text { Located in Building } 241-2 \text { Corridor. }\end{array}$ & $\begin{array}{l}\text { H-2-91823 Part No. } 13 \\
67.5^{\prime \prime} \text { H } \times 22 " \text { "W with } 61.25 " \\
\text { high panel mount ing space } \\
\text { for } 19 " \text { wide panels per ANSI } \\
\text { c83.9, rear door for access. }\end{array}$ & $\begin{array}{l}H-2-28883 \text { sht } 1 \\
H-2-28883 \text { sht } 2 \\
H-2-91823 \text { sht } 2\end{array}$ \\
\hline CBS-28-1 & $\begin{array}{l}\text { Central Battery system. Nominal } 24 \text { VDC } \\
\text { system. Includes one } 12 \text { cell battery } \\
\text { capable of supplying power to the } \\
\text { criticality alarm system in excess of } 8 \\
\text { hours and charger capable of maintaining the } \\
\text { charge on the battery and while providing } \\
\text { normal power. Primary supply power failure } \\
\text { annunciation. Located adjacent to CAP-z8. }\end{array}$ & $\begin{array}{l}\text { Battery: Exide } 82329 \\
\text { Charger: Exide } 101620\end{array}$ & $\begin{array}{l}H-2-28883 \text { sht } 1 \\
H-2-28883 \text { sht } 2 \\
H-2-91823 \text { sht } 2\end{array}$ \\
\hline $\begin{array}{l}\text { AC POWER RELAY } \\
\text { (Not required } \\
\text { for labeling } \\
\text { at this time.) }\end{array}$ & $\begin{array}{l}\text { (SC-3 SE). AC Power Monitor Relay. } \\
\text { Deenergizes on loss of power. A manual } \\
\text { reset is required to prevent a fluctuating } \\
\text { (erratic) power supply from initiating false } \\
\text { alarms. }\end{array}$ & $\begin{array}{l}\text { Square D Class } 8501 \\
\text { Catalog } \# \mathrm{CO}-16 \\
120 \text { VAC, } 60 \mathrm{~Hz}\end{array}$ & $\begin{array}{l}H-2-28883 \text { sht } 1 \\
H-2-28883 \text { sht } 2 \\
H-2-91823 \text { sht } 2\end{array}$ \\
\hline$S-8-1 A$ & $\begin{array}{l}\text { Inhibit switch for CAD/CADM-Z8-1A used to } \\
\text { prevent annunciation of alarm during test, } \\
\text { cal ibration, and replacement of CADS and } \\
\text { CADMs. Located on CAP-Z8. }\end{array}$ & $\begin{array}{l}\text { Square D Class } 9001 \text {, } \\
\text { Type LRSA-2K1 with } \\
\text { Type LA- } 1 \text { Contact Blocks }\end{array}$ & $\begin{array}{l}H-2-28883 \text { sht } 1 \\
H-2-28883 \text { sht } 2 \\
H-2-91823 \text { sht } 2\end{array}$ \\
\hline
\end{tabular}




\begin{tabular}{|c|c|c|c|}
\hline \multirow{2}{*}{\multicolumn{4}{|c|}{ 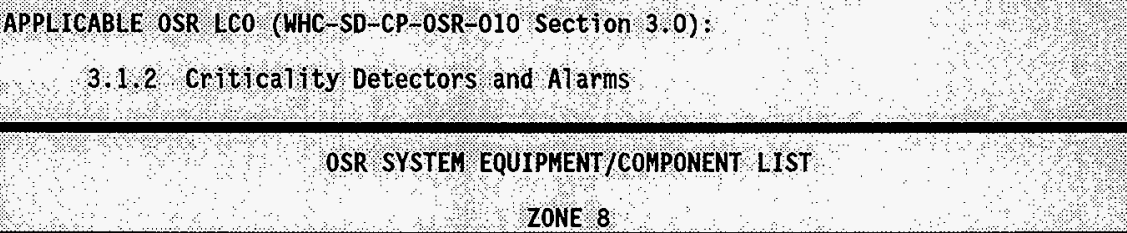 }} \\
\hline & & & \\
\hline $\begin{array}{l}\text { MASTER } \\
\text { CONPONENT } \\
\text { INDEX } \\
\text { NUMBER }\end{array}$ & $\begin{array}{l}\text { PUECTIONAL } \\
\text { DESCRIPTION }\end{array}$ & $\begin{array}{l}\text { MANUFACTURER } \\
\text { PARTIYODEL } \\
\text { NUNBER } \\
\text { (DWGH }=2-91823 \text { ) }\end{array}$ & $\begin{array}{l}\text { ESSENTAL } \\
\text { DRAMTNG } \\
\text { NUMBER }\end{array}$ \\
\hline$s-8-1 C$ & $\begin{array}{l}\text { Inhibit Switch for CAD/CADM-Z8-1C used to } \\
\text { prevent annunciation of alarm during test, } \\
\text { calibrat ion, and replacement of CADs and } \\
\text { CADMs. Located on CAP-Z8. }\end{array}$ & $\begin{array}{l}\text { Square D Class } 9001 \\
\text { Type LRSA-2K1 with } \\
\text { Type LA-1 Contact Blocks }\end{array}$ & $\begin{array}{l}H-2-28883 \text { sht } 1 \\
H-2-28883 \text { sht } 2 \\
H-2-91823 \text { sht } 2\end{array}$ \\
\hline$\$-8-2 A$ & $\begin{array}{l}\text { Inhibit switch for CAD/CADM-Z8-2A used to } \\
\text { prevent annunciation of alarm during test, } \\
\text { cal ibration, and replacement of CADS and } \\
\text { CADMs. Located on CAP-Z8. }\end{array}$ & $\begin{array}{l}\text { Square D Class } 9001 \\
\text { Type LRSA-2K1 with } \\
\text { Type LA-1 Contact Blocks }\end{array}$ & $\begin{array}{l}H-2-28883 \text { sht } 1 \\
H-2-28883 \text { sht } 2 \\
H-2-91823 \text { sht } 2\end{array}$ \\
\hline
\end{tabular}




\begin{tabular}{|c|c|c|c|}
\hline \multirow{2}{*}{ APP I CAB E } & \multicolumn{2}{|c|}{ 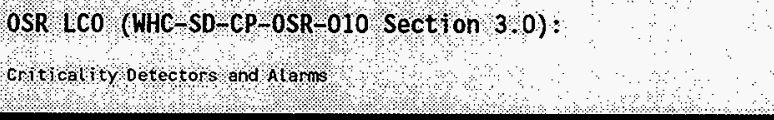 } & 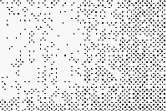 \\
\hline & 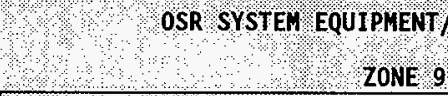 & MPONENT LIST & \\
\hline $\begin{array}{l}\text { MASTER } \\
\text { QOMPONENT } \\
\text { INDEX } \\
\text { WUMBER }\end{array}$ & FUNCTIONAL & $\begin{array}{l}\text { WANUFACTURER } \\
\text { PART J } \\
\text { NUNBER } \\
\text { (DWG H-2-80144/80145) }\end{array}$ & $\begin{array}{l}\text { ESSENHAL } \\
\text { ORAWING } \\
\text { NUMBER }\end{array}$ \\
\hline ASG-29. & $\begin{array}{l}\text { Audible Signal Generator. (Analog Type) } \\
\text { Generates an "AH-OO-GAH" electrical signal } \\
\text { that is wired to the criticality } \\
\text { annunciator horns. Located in CAP-Z9. }\end{array}$ & Fabricated per $\mathrm{H}-2-72419$ & $\begin{array}{l}H-2-28883 \text { sht } 1 \\
H-2-28883 \text { sht } 2 \\
H-2-80143 \text { sht } 2\end{array}$ \\
\hline$C A D-29-1 A$ & $\begin{array}{l}\text { Criticality Alarm Detector. (Gamma). } \\
\text { Detects radiation resulting from potential } \\
\text { criticality excursions. Located in } \\
\text { Building } 2736-28 \text {, Room } 642 \text {. }\end{array}$ & $\begin{array}{l}\text { Nuclear Measurement Corp. } \\
\text { Model GA-2 TMO }\end{array}$ & $\begin{array}{l}H-2-28883 \text { sht } 1 \\
H-2-28883 \text { sht } 2 \\
H-2-80143 \text { sht } 2\end{array}$ \\
\hline$C A D-Z 9-1 B$ & $\begin{array}{l}\text { Criticality Alarm Detector. (Gamma). } \\
\text { Detects radiation resulting from potential } \\
\text { criticality excursions. Located in } \\
\text { Building } 2736-2 B \text {, Room } 642 \text {. }\end{array}$ & $\begin{array}{l}\text { Nuclear Measurement Corp. } \\
\text { Model GA-2 TMO }\end{array}$ & $\begin{array}{l}H-2-28883 \text { sht } 1 \\
H-2-28883 \text { sht } 2 \\
H-2-80143 \text { sht } 2\end{array}$ \\
\hline$C A D-29-1 C$ & $\begin{array}{l}\text { Criticality Alarm Detector. (Gamma). } \\
\text { Detects radiation resulting from potential } \\
\text { criticality excursions. Located in } \\
\text { Building } 2736-28 \text {, Room } 642 \text {. }\end{array}$ & $\begin{array}{l}\text { Nuclear Measurement Corp. } \\
\text { Hodel GA-2 TMO }\end{array}$ & $\begin{array}{l}H-2-28883 \text { sht } 1 \\
H-2-28883 \text { sht } 2 \\
H-2-80143 \text { sht } 2\end{array}$ \\
\hline$C A D-29-2 A$ & $\begin{array}{l}\text { Criticality Alarm Detector. (Gamma). } \\
\text { Detects radiation resulting from potential } \\
\text { criticality excursions. Located in } \\
\text { Building } 2736-2 B \text {, Room } 641 \text {. }\end{array}$ & $\begin{array}{l}\text { Nuclear Measurement Corp. } \\
\text { Model GA-2 TMO }\end{array}$ & $\begin{array}{l}H-2-28883 \text { sht } 1 \\
H-2-28883 \text { sht } 2 \\
H-2-80143 \text { sht } 2\end{array}$ \\
\hline$C A D-Z 9-2 B$ & $\begin{array}{l}\text { Criticality Alarm Detector. (Gamma). } \\
\text { Detects radiation resulting from potential } \\
\text { criticality excursions. Located in } \\
\text { Building } 2736-2 B \text {, Room } 641 \text {. }\end{array}$ & $\begin{array}{l}\text { Nuclear Measurement Corp. } \\
\text { Model GA-2 TMO }\end{array}$ & $\begin{array}{l}H-2-28883 \text { sht } 1 \\
H-2-28883 \text { sht } 2 \\
H-2-80143 \text { sht } 2\end{array}$ \\
\hline$C A D-29-2 C$ & $\begin{array}{l}\text { Criticality Alarm Detector. (Gamma). } \\
\text { Detects radiation resulting from potential } \\
\text { criticality excursions. Located in } \\
\text { Building } 2736-2 B \text {, Room } 641 \text {. }\end{array}$ & $\begin{array}{l}\text { Nuclear Measurement Corp. } \\
\text { Model GA-2 TMO }\end{array}$ & $\begin{array}{l}H-2-28883 \text { sht } 1 \\
H-2-28883 \text { sht } 2 \\
H-2-80143 \text { sht } 2\end{array}$ \\
\hline$C A D-29-3 A$ & $\begin{array}{l}\text { Criticality Alarm Detector. (Gamma). } \\
\text { Detects radiation resulting from potential } \\
\text { criticality excursions. Located in } \\
\text { Building_2736-2B, Roon } 638 \text {. }\end{array}$ & $\begin{array}{l}\text { Nuclear Measurement Corp. } \\
\text { Model GA-2 TMO }\end{array}$ & $\begin{array}{l}H-2-28883 \text { sht } 1 \\
H-2-28883 \text { sht } 2 \\
H-2-80143 \text { sht } 2\end{array}$ \\
\hline$C A D-29-3 B$ & $\begin{array}{l}\text { Criticality Alarm Detector. (Ganma). } \\
\text { Detects radiation resulting from potential } \\
\text { criticality excursions. Located in } \\
\text { Building } 2736-2 B \text {, Room } 638 \text {. }\end{array}$ & $\begin{array}{l}\text { Nuclear Measurement Corp. } \\
\text { Model GA-2 TMO }\end{array}$ & $\begin{array}{l}H-2-28883 \text { sht } 1 \\
H-2-28883 \text { sht } 2 \\
H-2-80143 \text { sht } 2\end{array}$ \\
\hline$C A D-29-3 C$ & $\begin{array}{l}\text { Criticality Alarm Detector. (Gamma). } \\
\text { Detects radiation resulting from potential } \\
\text { criticality excursions. Located in } \\
\text { Building } 2736-2 B \text {, Room } 638 \text {. }\end{array}$ & $\begin{array}{l}\text { Nuclear Measurement Corp. } \\
\text { Model GA-2 TMO }\end{array}$ & $\begin{array}{l}H-2-28883 \text { sht } 1 \\
H-2-28883 \text { sht } 2 \\
H-2-80143 \text { sht } 2\end{array}$ \\
\hline$C A D-29-4 A$ & $\begin{array}{l}\text { Criticality Atarm Detector. (Gamma). } \\
\text { Detects radiation resulting from potential } \\
\text { criticality excursions. Located in } \\
\text { Building } 2736-2 B \text {, Room } 636 \text {. }\end{array}$ & $\begin{array}{l}\text { Nuclear Measurement Corp. } \\
\text { Model GA-2 TMO }\end{array}$ & $\begin{array}{l}H-2-28883 \text { sht } 1 \\
H-2-28883 \text { sht } 2 \\
H-2-80143 \text { sht } 2\end{array}$ \\
\hline
\end{tabular}




\begin{tabular}{|c|c|c|c|}
\hline \multirow{2}{*}{ APP L CABL } & \multicolumn{2}{|c|}{ 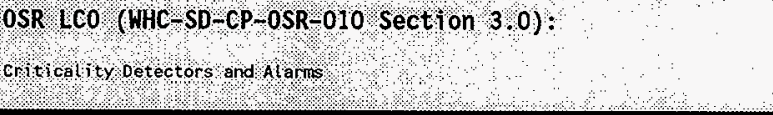 } & \\
\hline & OOSR SYSTEN EQUTPNENT & OHPONENT LIST & \\
\hline $\begin{array}{l}\text { MASTER } \\
\text { COMPONEAT } \\
\text { WWDEX } \\
\text { WUWBER }\end{array}$ & FUNCTIONAL & $\begin{array}{l}\text { MANUFACTURER } \\
\text { PART/NODEL } \\
\text { NUMBER } \\
\text { (DWG H-2-80144/80145) }\end{array}$ & $\begin{array}{l}\text { ESSENTAL } \\
\text { DRAWNAG } \\
\text { WHBER }\end{array}$ \\
\hline$C A D-29-4 B$ & $\begin{array}{l}\text { Criticality Alarm Detector. (Gamma). } \\
\text { Detects radiation resulting from potential } \\
\text { criticality excursions. Located in } \\
\text { Building } 2736-2 B \text {, Room } 636 \text {. }\end{array}$ & $\begin{array}{l}\text { Nucl ear Measurement corp. } \\
\text { Model GA-2 TMO }\end{array}$ & $\begin{array}{l}H-2-28883 \text { sht } 1 \\
H-2-28883 \text { sht } 2 \\
H-2-80143 \text { sht } 2\end{array}$ \\
\hline$C A D-29-4 C$ & $\begin{array}{l}\text { Criticality Alarm Detector. (Gamma). } \\
\text { Detects radiation resulting from potential } \\
\text { criticality excursions. Located in } \\
\text { Building } 2736-28 \text {, Room } 636 \text {. }\end{array}$ & $\begin{array}{l}\text { Nuclear Measurement Corp. } \\
\text { Model GA-2 TMO }\end{array}$ & $\begin{array}{l}H-2-28883 \text { sht } 1 \\
H-2-28883 \text { sht } 2 \\
H-2-80143 \text { sht } 2\end{array}$ \\
\hline CAD- $29-5 A$ & $\begin{array}{l}\text { Criticality Alarm Detector. (Gamma). } \\
\text { Detects radiation resulting from potential } \\
\text { criticality excursions. Located in } \\
\text { Building } 2736-2 B \text {, Room } 637 \text {. }\end{array}$ & $\begin{array}{l}\text { Nuclear Measurement Corp. } \\
\text { Hodel GA-2 TMO }\end{array}$ & $\begin{array}{l}H-2-28883 \text { sht } 1 \\
H-2-28883 \text { sht } 2 \\
H-2-80143 \text { sht } 2\end{array}$ \\
\hline$C A D-29-5 B$ & $\begin{array}{l}\text { Criticality Alarm Detector. (Gamma). } \\
\text { Detects radiation resulting from potential } \\
\text { criticality excursions. Located in } \\
\text { Building } 2736-2 B \text {, Room } 637 \text {. }\end{array}$ & $\begin{array}{l}\text { Nuclear Measurement corp. } \\
\text { Model GA-2 TMO }\end{array}$ & $\begin{array}{l}H-2-28883 \text { sht } 1 \\
H-2-28883 \text { sht } 2 \\
H-2-80143 \text { sht } 2\end{array}$ \\
\hline$C A D-29-5 C$ & $\begin{array}{l}\text { Criticality Alarm Detector. (Gamma). } \\
\text { Detects radiation resulting from potential } \\
\text { criticality excursions. Located in } \\
\text { Building } 2736-2 B \text {, Room } 637 \text {. }\end{array}$ & $\begin{array}{l}\text { Nuclear Measurement Corp. } \\
\text { Model GA-2 TMO }\end{array}$ & $\begin{array}{l}H-2-28883 \text { sht } 1 \\
H-2-28883 \text { sht } 2 \\
H-2-80143 \text { sht } 2\end{array}$ \\
\hline CADM-29-1A & $\begin{array}{l}\text { Criticality Alarm Detection Module. One } \\
\text { (1) per CAD. Provides dose rate } \\
\text { indication, calibration adjustments for } \\
\text { the CAD-CADM Unit, and alarm functions. } \\
\text { Located in CAP-Z9. }\end{array}$ & $\begin{array}{l}\text { Nuclear Measurement Corp. } \\
\text { Model GA-2 TMO }\end{array}$ & $\begin{array}{l}H-2-28883 \text { sht } t \\
H-2-28883 \text { sht } 2 \\
H-2-80143 \text { sht } 2\end{array}$ \\
\hline CADM-29-1B & $\begin{array}{l}\text { Criticality Alarm Detection Module. One } \\
\text { (1) per CAD. Provides dose rate } \\
\text { indication, cal ibration adjustments for } \\
\text { the CAD-CADM unit, and alarm functions. } \\
\text { Located in CAP-Z9. }\end{array}$ & $\begin{array}{l}\text { Nuclear Measurement Corp. } \\
\text { Model GA-2 YMO }\end{array}$ & $\begin{array}{l}H-2-28883 \text { sht } 1 \\
H-2-28883 \text { sht } 2 \\
H-2-80143 \text { sht } 2\end{array}$ \\
\hline CADM-29-1C & $\begin{array}{l}\text { Criticality Alarm Detection Module. One } \\
\text { (1) per CAD. Provides dose rate } \\
\text { indication, cal ibration adjustments for } \\
\text { the CAD-CADM unit, and alarm functions. } \\
\text { Located in CAP- } 29 \text {. }\end{array}$ & $\begin{array}{l}\text { Nuclear Measurement Corp. } \\
\text { Model GA-2 TMO }\end{array}$ & $\begin{array}{l}H-2-28883 \text { sht } 1 \\
H-2-28883 \text { sht } 2 \\
H-2-80143 \text { sht } 2\end{array}$ \\
\hline CADM-Z9-2A & $\begin{array}{l}\text { Criticality Alarm Detection Module. One } \\
\text { (1) per CAD. Provides dose rate } \\
\text { indication, cal ibration adjustments for } \\
\text { the CAD-CADM unit, and alarm functions. } \\
\text { Located in CAP- } 29 \text {. }\end{array}$ & $\begin{array}{l}\text { Nuclear Measurement Corp. } \\
\text { Model GA-2 TMO }\end{array}$ & $\begin{array}{l}H-2-28883 \text { sht } 1 \\
H-2-28883 \text { sht } 2 \\
H-2-80143 \text { sht } 2\end{array}$ \\
\hline CADM-Z9-2B & $\begin{array}{l}\text { Criticality Alarm Detection Module. One } \\
\text { (1) per CAD. Provides dose rate } \\
\text { indication, calibration adjustments for } \\
\text { the CAD-CADM unit, and alarm functions. } \\
\text { Located in CAP- } 29 \text {. }\end{array}$ & $\begin{array}{l}\text { Nuclear Measurement Corp. } \\
\text { Model GA-2 TMO }\end{array}$ & $\begin{array}{l}H-2-28883 \text { sht } 1 \\
H-2-28883 \text { sht } 2 \\
H-2-80143 \text { sht } 2\end{array}$ \\
\hline
\end{tabular}




\begin{tabular}{|c|c|c|c|}
\hline \multicolumn{4}{|c|}{ 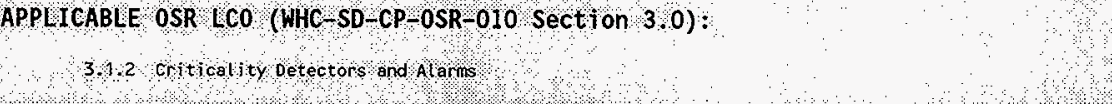 } \\
\hline 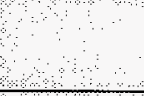 & OSR SYSTEM EQUTPME & OHPONENTLIST & \\
\hline $\begin{array}{l}\text { MASTER } \\
\text { COMPONENT } \\
\text { NOMDEX }\end{array}$ & $\begin{array}{ll}\text { SFUNCTIONAL } & \\
\text { DESCRIPTION } & \end{array}$ & $\begin{array}{l}\text { WANUFACTURER } \\
\text { PART JWODEL } \\
\text { NUWBER } \\
\left.\text { (DWG } \mathrm{H}_{2} 2,80144 / 80145\right)\end{array}$ & $\begin{array}{l}\text { SSENT AL } \\
\text { DRAWH } \\
\text { NGMBER }\end{array}$ \\
\hline CADM-29-2C & $\begin{array}{l}\text { Criticality Alarm Detection Module. One } \\
\text { (1) per CAD. Provides dose rate } \\
\text { indication, calibration adjustments for } \\
\text { the CAD-CADM Unit, and alarm functions. } \\
\text { Located in CAP-Z9. }\end{array}$ & $\begin{array}{l}\text { Nuclear Measurement Corp. } \\
\text { Model GA-2 TMO }\end{array}$ & $\begin{array}{l}H-2-28883 \text { sht } 1 \\
H-2-28883 \text { sht } 2 \\
H-2-80143 \text { sht } 2\end{array}$ \\
\hline CADM-Z9-3A & $\begin{array}{l}\text { Criticality Alarm Detection Module. One } \\
\text { (1) per CAD. Provides dose rate } \\
\text { indication, calibration adjustments for } \\
\text { the CAD-CADM unit, and alarm functions. } \\
\text { Located in CAP-Z9. }\end{array}$ & $\begin{array}{l}\text { Nuclear Measurement Corp. } \\
\text { Model GA-2 TMO }\end{array}$ & $\begin{array}{l}H-2-28883 \text { sht } 1 \\
H-2-28883 \text { sht } 2 \\
H-2-80143 \text { sht } 2\end{array}$ \\
\hline CADM-29-3B & $\begin{array}{l}\text { Criticality Alarm Detection Module. One } \\
\text { (1) per CAD. Provides dose rate } \\
\text { indication, calibration adjustments for } \\
\text { the CAD-CADM unit, and alarm functions. } \\
\text { Located in CAP-Z9. }\end{array}$ & $\begin{array}{l}\text { Nuclear Heasurement Corp. } \\
\text { Model GA-2 TMO }\end{array}$ & $\begin{array}{l}H-2-28883 \text { sht } 1 \\
H-2-28883 \text { sht } 2 \\
H-2-80143 \text { sht } 2\end{array}$ \\
\hline CADM $-29-3 C$ & $\begin{array}{l}\text { Criticality Alarm Detection Module. one } \\
\text { (1) per CAD. Provides dose rate } \\
\text { indication, cal ibration adjustments for } \\
\text { the CAD-CADM unit, and alarm functions. } \\
\text { Located in CAP-Z9. }\end{array}$ & $\begin{array}{l}\text { Nuclear Measurement Corp. } \\
\text { Model GA-2 TMO }\end{array}$ & $\begin{array}{l}H-2-28883 \text { sht } 1 \\
H-2-28883 \text { sht } 2 \\
H-2-80143 \text { sht } 2\end{array}$ \\
\hline CADM-29-4A & $\begin{array}{l}\text { Criticality Alarm Detection Module. One } \\
\text { (1) per CAD. Provides dose rate } \\
\text { indication, calibration adjustments for } \\
\text { the CAD-CADM Unit, and alarm functions. } \\
\text { Located in CAP- } 29 \text {. }\end{array}$ & $\begin{array}{l}\text { Nuclear Measurement Corp. } \\
\text { Model GA-2 TMO }\end{array}$ & $\begin{array}{l}H-2-28883 \text { sht ? } \\
H-2-28883 \text { sht } 2 \\
H-2-80143 \text { sht } 2\end{array}$ \\
\hline CADM- $29-4 B$ & $\begin{array}{l}\text { Criticality Alarm Detection Module. One } \\
\text { (1) per CAD. Provides dose rate } \\
\text { indication, cal ibration adjustments for } \\
\text { the CAD-CADM unit, and alarm functions. } \\
\text { Located in CAP-Z9. }\end{array}$ & $\begin{array}{l}\text { Nuclear Measurement Corp. } \\
\text { Model GA-2 TMO }\end{array}$ & $\begin{array}{l}H-2-28883 \text { sht } 1 \\
H-2-28883 \text { sht } 2 \\
H-2-80143 \text { sht } 2\end{array}$ \\
\hline CADM- $29-4 C$ & $\begin{array}{l}\text { Criticality Alarm Detection Module. One } \\
\text { (1) per CAD. Provides dose rate } \\
\text { indication, cal ibration adjustments for } \\
\text { the CAD-CADH unit, and alarm functions. } \\
\text { Located in CAP-29. }\end{array}$ & $\begin{array}{l}\text { Nucl ear Measurement Corp. } \\
\text { Model GA-2 TMO }\end{array}$ & $\begin{array}{l}H-2-28883 \text { sht } 1 \\
H-2-28883 \text { sht } 2 \\
H-2-80143 \text { sht } 2\end{array}$ \\
\hline CADM-Z9-5A & $\begin{array}{l}\text { Critical ity Alarm Detection Module. One } \\
\text { (1) per CAD. Provides dose rate } \\
\text { indication, cal ibration adjustments for } \\
\text { the CAD-CADM unit, and alarm functions. } \\
\text { Located in CAP- } 29 \text {. }\end{array}$ & $\begin{array}{l}\text { Nucl ear Measurement Corp. } \\
\text { Model GA-2 TMO }\end{array}$ & $\begin{array}{l}H-2-28883 \text { sht } 1 \\
H-2-28883 \text { sht } 2 \\
H-2-80143 \text { sht } 2\end{array}$ \\
\hline CADM-Z9-5B & $\begin{array}{l}\text { Criticality Alarm Detection Module. One } \\
\text { (1) per CAD. Provides dose rate } \\
\text { indicat ion, calibration adjustments for } \\
\text { the CAD-CADM unit, and alarm functions. } \\
\text { Located in CAP-Z9. }\end{array}$ & $\begin{array}{l}\text { Nucl ear Measurement Corp. } \\
\text { Model GA-2 TMO }\end{array}$ & $\begin{array}{l}H-2-28883 \text { sht } 1 \\
H-2-28883 \text { sht } 2 \\
H-2-80143 \text { sht } 2\end{array}$ \\
\hline
\end{tabular}




\begin{tabular}{|c|c|c|c|}
\hline \multirow[t]{2}{*}{ APPICABLE } & \multicolumn{3}{|c|}{ 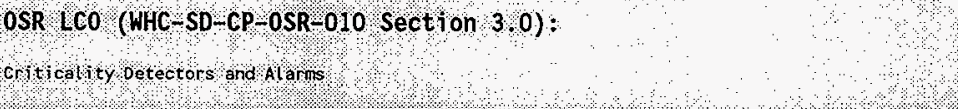 } \\
\hline & OOSR SYSTEM EQUTPNEN & MPONENT LIST & \\
\hline $\begin{array}{l}\text { WOASTER } \\
\text { COMPONENT } \\
\text { WUMBEX }\end{array}$ & 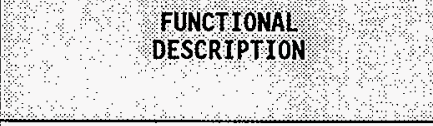 & $\begin{array}{l}\text { WANUFACTURER } \\
\text { PART } \\
\text { NUMBER } \\
\text { (DWG }\end{array}$ & $\begin{array}{l}\text { ESSENTAL } \\
\text { DRAWING }\end{array}$ \\
\hline CADM-29-5C & $\begin{array}{l}\text { Criticality Alarm Detection Module. One } \\
\text { (1) per CAD. Provides dose rate } \\
\text { indication, calibration adjustments for } \\
\text { the CAD-CADH unit, and alarm functions. } \\
\text { Located in CAP-Z9. }\end{array}$ & $\begin{array}{l}\text { Nuclear Measurement corp. } \\
\text { Model GA-2 TMO }\end{array}$ & $\begin{array}{l}H-2-28883 \text { sht } 1 \\
H-2-28883 \text { sht } 2 \\
H-2-80143 \text { sht } 2\end{array}$ \\
\hline CAF-29-1 & $\begin{array}{l}\text { Criticality Alarm Flasher Rotating. } \\
\text { Located in Building } 2721-\text { - B Room } 648 .\end{array}$ & $\begin{array}{l}\text { Federal Signal Corp. Model } \\
121 \mathrm{~A}, \mathrm{Bl} \text { lue }\end{array}$ & $\begin{array}{l}H-2-28883 \text { sht } 1 \\
H-2-28883 \text { sht } 2 \\
H-2-80143 \text { sht } 2 \\
\end{array}$ \\
\hline CAH $-29-1$ & $\begin{array}{l}\text { Criticality Alarm Horn. Located in } \\
\text { Building } 2736-28 \text { Corridor } 625 \text {. }\end{array}$ & $\begin{array}{l}\text { Audible Signaling Device: } \\
\text { Federal Signal Corp. Model } \\
300 \text { GC, } 24 \text { VAC/DC Selectone } \\
\text { Connector Board: FS Corp. } \\
\text { Model } 300 \text { CK }\end{array}$ & $\begin{array}{l}H-2-28883 \text { sht } 1 \\
H-2-28883 \text { sht } 2 \\
H-2-80143 \text { sht } 2\end{array}$ \\
\hline $\mathrm{CAH}-29-2$ & $\begin{array}{l}\text { Criticality Alarm Horn. Located in } \\
\text { Building } 2736-2 B \text { Corridor } 625 \text {. }\end{array}$ & $\begin{array}{l}\text { Audible signal ing Device: } \\
\text { Federal signal Corp. Model } \\
300 \mathrm{GC}, 24 \text { VAC/DC Selectone } \\
\text { Connector Board: FS Corp. } \\
\text { Model } 300 \text { ck }\end{array}$ & $\begin{array}{l}H-2-28883 \text { sht } 1 \\
H-2-28883 \text { sht } 2 \\
H-2-80143 \text { sht } 2\end{array}$ \\
\hline САH-Z9-3 & $\begin{array}{l}\text { Criticality Alarm Horn. Located in } \\
\text { Building } 2736-28 \text { Room } 641 \text {. }\end{array}$ & $\begin{array}{l}\text { Audible signal ing Device: } \\
\text { Federal signal Corp. Hodel } \\
300 \mathrm{GC}, 24 \mathrm{VAC} / \mathrm{DC} \text { Selectone } \\
\text { Connector Board: FS Corp. } \\
\text { Model } 300 \mathrm{CK} \\
\end{array}$ & $\begin{array}{l}H-2-28883 \text { sht } 1 \\
H-2-28883 \text { sht } 2 \\
H-2-80143 \text { sht } 2\end{array}$ \\
\hline $\mathrm{CAH}-29-4$ & $\begin{array}{l}\text { Criticality Alarm Horn. Located in } \\
\text { Building } 2736-2 B \text { Room } 641 \text {. }\end{array}$ & $\begin{array}{l}\text { Audible signaling Device: } \\
\text { Federal signal Corp. Model } \\
300 \mathrm{GC}, 24 \text { VAC/DC Selectone } \\
\text { Connector Board: FS Corp. } \\
\text { Model } 300 \text { CK }\end{array}$ & $\begin{array}{l}H-2-28883 \text { sht } 1 \\
H-2-28883 \text { sht } 2 \\
H-2-80143 \text { sht } 2\end{array}$ \\
\hline CAH-Z9-5 & $\begin{array}{l}\text { Criticality Alarm Horn. Located in } \\
\text { Building } 2736-28 \text { Room } 637 \text {. }\end{array}$ & $\begin{array}{l}\text { Audible Signaling Device: } \\
\text { Federal signal Corp. Model } \\
300 \mathrm{GC}, 24 \text { VAC/DC Selectone } \\
\text { Connector Board: FS Corp. } \\
\text { Model } 300 \text { CK }\end{array}$ & $\begin{array}{l}H-2-28883 \text { sht } 1 \\
H-2-28883 \text { sht } 2 \\
H-2-80143 \text { sht } 2\end{array}$ \\
\hline $\mathrm{CAH}-29-6$ & $\begin{array}{l}\text { Criticality Alarm Horn. Located in } \\
\text { Building } 2736-28 \text { Room } 637 \text {. }\end{array}$ & $\begin{array}{l}\text { Audible signaling Device: } \\
\text { Federal signal Corp. Model } \\
300 \text { GC, } 24 \text { VAC/DC Selectone } \\
\text { Connector Board: FS Corp. } \\
\text { Model } 300 \text { CK }\end{array}$ & $\begin{array}{l}H-2-28883 \text { sht } 1 \\
H-2-28883 \text { sht } 2 \\
H-2-80143 \text { sht } 2\end{array}$ \\
\hline CAH-Z9-7 & $\begin{array}{l}\text { Critical ity Alarm Horn. Located in } \\
\text { Building } 2736-28 \text { Room } 622 \text {. }\end{array}$ & $\begin{array}{l}\text { Audible signaling Device: } \\
\text { Federal Signal Corp. Model } \\
300 \mathrm{GC}, 24 \text { VAC/DC Selectone } \\
\text { Connector Board: FS Corp. } \\
\text { Model } 300 \text { CK }\end{array}$ & $\begin{array}{l}\text { H-2-2-28883 sht } 1 \\
\text { H-2-28883 sht } 2 \\
\text { H-2-80143 sht } 2\end{array}$ \\
\hline
\end{tabular}


DEFINITION AND MEANS OF MAINTAINING THE CRITICALITY DETECTORS AND ALARMS PORTION

\begin{tabular}{|c|c|c|c|}
\hline \multicolumn{4}{|c|}{ 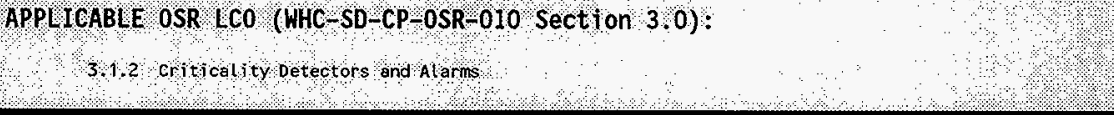 } \\
\hline सा & OSR SYSTEN EQUI & DHPONENT LIST & \\
\hline $\begin{array}{l}\text { MASTER } \\
\text { COMPONENT } \\
\text { WHOMBER }\end{array}$ & 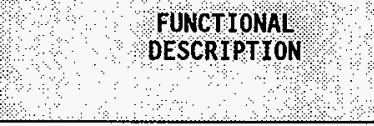 & $\begin{array}{l}\text { MANUFACTURER } \\
\text { PART/HODEL } \\
\text { NUMBER } \\
\text { (DWG } \mathrm{H}-2-80144 / 80145)\end{array}$ & ESSENTAL \\
\hline CAH-29-8 & $\begin{array}{l}\text { Criticality Alarm Horn. Located in } \\
\text { Building } 2736-2 B \text { Room } 622 \text {. }\end{array}$ & $\begin{array}{l}\text { Audible Signaling Device: } \\
\text { Federal Signal Corp. Model } \\
300 \mathrm{GC}, 24 \text { VAC/DC Selectone } \\
\text { Connector Board: FS Corp. } \\
\text { Model } 300 \text { CK }\end{array}$ & $\begin{array}{l}H-2-28883 \text { sht } 1 \\
H-2-28883 \text { sht } 2 \\
H-2-80143 \text { sht } 2\end{array}$ \\
\hline $\mathrm{CAH}-\mathrm{Z9-9}$ & $\begin{array}{l}\text { Criticality Alarm Horn. Located in } \\
\text { Building } 2736-2 B \text { Room } 600 \text {. }\end{array}$ & $\begin{array}{l}\text { Audible signal ing Device: } \\
\text { Federal Signal Corp. Model } \\
300 \text { GC, } 24 \text { VAC/DC Selectone } \\
\text { Connector Board: FS Corp. } \\
\text { Model } 300 \text { CK }\end{array}$ & $\begin{array}{l}H-2-28883 \text { sht } 1 \\
H-2-28883 \text { sht } 2 \\
H-2-80143 \text { sht } 2\end{array}$ \\
\hline$C A H-29-10$ & $\begin{array}{l}\text { Criticality Alarm Horn. Located in } \\
\text { Building } 2736-2 B \text { Room } 600 \text {. }\end{array}$ & $\begin{array}{l}\text { Audible Signaling Device: } \\
\text { Federal Signal Corp. Model } \\
300 \text { GC, } 24 \text { VAC/DC Selectone } \\
\text { Connector Board: FS Corp. } \\
\text { Model } 300 \text { CK }\end{array}$ & $\begin{array}{l}H-2-28883 \text { sht } 1 \\
H-2-28883 \text { sht } 2 \\
H-2-80143 \text { sht } 2\end{array}$ \\
\hline CAH-29-11 & $\begin{array}{l}\text { Criticality Alarm Horn. Located in } \\
\text { Building } 2736-28 \text { Room } 602 \text {. }\end{array}$ & $\begin{array}{l}\text { Audible Signaling Device: } \\
\text { Federal signal Corp. Model } \\
300 \text { GC, } 24 \text { VAC/DC Selectone } \\
\text { Connect or Board: FS Corp. } \\
\text { Model } 300 \text { CK }\end{array}$ & $\begin{array}{l}H-2-28883 \text { sht } 1 \\
H-2-28883 \text { sht } 2 \\
H-2-80143 \text { sht } 2\end{array}$ \\
\hline CAH $-29-12$ & $\begin{array}{l}\text { Criticality Alarm Horn. Located in } \\
\text { Building } 2736-28 \text { Room } 602 \text {. }\end{array}$ & $\begin{array}{l}\text { Audible signal ing Device: } \\
\text { Federal Signal Corp. Model } \\
300 \text { GC, } 24 \text { VAC/DC Selectone } \\
\text { Connectior Board: FS Corp. } \\
\text { Model } 300 \text { CK }\end{array}$ & $\begin{array}{l}H-2-28883 \text { sht } 1 \\
\text { H-2-28883 sht } 2 \\
\text { H-2-80143 sht } 2\end{array}$ \\
\hline $\mathrm{CAH}-29-13$ & $\begin{array}{l}\text { Criticality Alarm Horn. Located in } \\
\text { Building } 2736-2 B \text { Room } 620 \text {. }\end{array}$ & $\begin{array}{l}\text { Audible Signaling Device: } \\
\text { Federal signal Corp. Model } \\
300 \text { GC, } 24 \text { VAC/DC Selectone } \\
\text { Connector Board: FS Corp. } \\
\text { Model } 300 \text { CK }\end{array}$ & $\begin{array}{l}H-2-28883 \text { sht } 1 \\
H-2-28883 \text { sht } 2 \\
H-2-80143 \text { sht } 2\end{array}$ \\
\hline CAH-Z9- 14 & $\begin{array}{l}\text { Criticality Alarm Horn. Located in } \\
\text { Building } 2736-28 \text { Room } 620 \text {. }\end{array}$ & $\begin{array}{l}\text { Audible Signal ing Device: } \\
\text { Federal Signal Corp. Model } \\
300 \mathrm{GC}, 24 \text { VAC/DC Selectone } \\
\text { Connector Board: FS Corp. } \\
\text { Model } 300 \text { CK }\end{array}$ & $\begin{array}{l}H-2-28883 \text { sht } 1 \\
H-2-28883 \text { sht } 2 \\
H-2-80143 \text { sht } 2\end{array}$ \\
\hline CAH-29-15 & $\begin{array}{l}\text { Criticality Alarm Horn. Located in } \\
\text { Building } 2736-2 B \text { Corridor } 609 .\end{array}$ & $\begin{array}{l}\text { Audible Signaling Device: } \\
\text { Federal Signal Corp. Model } \\
300 \mathrm{GC}, 24 \text { VAC/DC Selectone } \\
\text { Connector Board: FS Corp. } \\
\text { Model } 300 \mathrm{CK}\end{array}$ & $\begin{array}{l}\text { H-2-28883 sht } 1 \\
H-2-28883 \text { sht } 2 \\
H-2-80143 \text { sht } 2\end{array}$ \\
\hline CAH-Z9-16 & $\begin{array}{l}\text { Criticality Alarm Horn. Located in } \\
\text { Building } 2736-2 B \text { Corridor } 609 .\end{array}$ & $\begin{array}{l}\text { Audible Signaling Device: } \\
\text { Federal Signal Corp. Model } \\
300 \mathrm{GC}, 24 \text { VAC/DC Selectone } \\
\text { Connector Board: FS Corp. } \\
\text { Model } 300 \mathrm{CK}\end{array}$ & $\begin{array}{l}\text { H-2-28883 sht } 1 \\
\text { H-2-28883 sht } 2 \\
\text { H-2-80143 sht } 2\end{array}$ \\
\hline
\end{tabular}




\begin{tabular}{|c|c|c|c|}
\hline \multicolumn{4}{|l|}{ 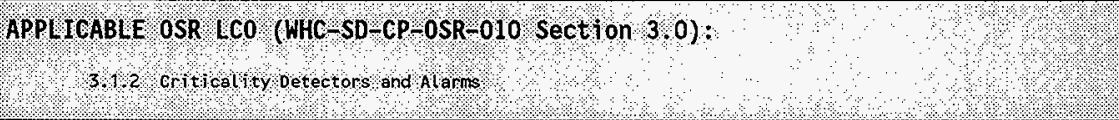 } \\
\hline 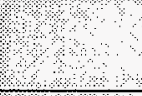 & $\begin{array}{l}\text { OSR SYSTEY EQUIPHEN } \\
\text { OSONE }\end{array}$ & OMPONENT LIST & \\
\hline $\begin{array}{l}\text { MASTER } \\
\text { COMPONENT } \\
\text { NUNDEX } \\
\end{array}$ & PDESCRTPTION & $\begin{array}{l}\text { MANUFACTURER } \\
\text { PART /NOEL } \\
\text { NUHBER } \\
\text { (DWOHH-2-80144/80H45) }\end{array}$ & 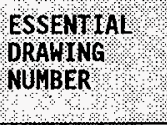 \\
\hline CAH- Z9-17 & $\begin{array}{l}\text { Criticality Alarm Horn. Located outside } \\
\text { on South wall of Building } 2736-2 B \text {. }\end{array}$ & $\begin{array}{l}\text { Audible Signaling Device: } \\
\text { Federal Signal Corp. Model } \\
300 \mathrm{GC}, 24 \text { VAC/DC Selectone } \\
\text { Connector Board: FS Corp. } \\
\text { Model } 300 \mathrm{CK}\end{array}$ & $\begin{array}{l}H-2-28883 \text { sht } 1 \\
H-2-28883 \text { sht } 2 \\
H-2-80143 \text { sht } 2\end{array}$ \\
\hline $\mathrm{CAH}-\mathrm{Z9}-18$ & $\begin{array}{l}\text { Criticality Alarm Horn. Located outside } \\
\text { on South Wall of Building } 2736-2 B \text {. }\end{array}$ & $\begin{array}{l}\text { Audible signaling Device: } \\
\text { Federal signal Corp. Model } \\
300 \mathrm{GC}, 24 \text { VAC/DC Selectone } \\
\text { Connector Board: FS Corp. } \\
\text { Model } 300 \mathrm{CK}\end{array}$ & $\begin{array}{l}H-2-28883 \text { sht } 1 \\
H-2-28883 \text { sht } 2 \\
H-2-80143 \text { sht } 2\end{array}$ \\
\hline CAH-29-19 & $\begin{array}{l}\text { Criticality Alarm Horn. Located outside } \\
\text { on East Wall of Building } 2736-2 \mathrm{~B} \text {. }\end{array}$ & $\begin{array}{l}\text { Audible Signal ing Device: } \\
\text { Federal Signal Corp. Model } \\
300 \text { GC, } 24 \text { VAC/DC Selectone } \\
\text { Connector Board: FS Corp. } \\
\text { Model } 300 \text { CK }\end{array}$ & $\begin{array}{l}H-2-28883 \text { sht } 1 \\
H-2-28883 \text { sht } 2 \\
H-2-80143 \text { sht } 2\end{array}$ \\
\hline САH- $29-20$ & $\begin{array}{l}\text { Criticality Alarm Horn. Located in } \\
\text { Building } 2721-28 \text { Room } 647 \text {. }\end{array}$ & $\begin{array}{l}\text { Audible signal ing Device: } \\
\text { Federal signal Corp. Model } \\
300 \text { GC, } 24 \text { VAC/DC Selectone } \\
\text { Connector Board: FS Corp. } \\
\text { Model } 300 \text { CK }\end{array}$ & $\begin{array}{l}H-2-28883 \text { sht } 1 \\
H-2-28883 \text { sht } 2 \\
H-2-80143 \text { sht } 2\end{array}$ \\
\hline САН-29-21 & $\begin{array}{l}\text { Criticality Alarm Horn. Located in } \\
\text { Building } 2721-28 \text { Room } 648 \text {. }\end{array}$ & $\begin{array}{l}\text { Audible Signal ing Device: } \\
\text { Federal Signal Corp. Hodel } \\
300 \text { GC, } 24 \text { VAC/DC Selectone } \\
\text { Connector Board: FS Corp. } \\
\text { Model } 300 \text { CK }\end{array}$ & $\begin{array}{l}H-2-28883 \text { sht } 1 \\
H-2-28883 \text { sht } 2 \\
H-2-80143 \text { sht } 2\end{array}$ \\
\hline CALM-29-1 & $\begin{array}{l}\text { Criticality Alarm Logic Modute. Provides } \\
\text { the logic circuitry for annunciation of } \\
\text { alert and alarm conditions as detected by } \\
\text { the CADMs. Located in CAP-29. }\end{array}$ & Per Drawing $\mathrm{H}-2-91179$ & $\begin{array}{l}H-2-28883 \text { sht } 1 \\
H-2-28883 \text { sht } 2 \\
H-2-80143 \text { sht } 2\end{array}$ \\
\hline CALM-Z9-2 & $\begin{array}{l}\text { Criticality Alarm Logic Module. Provides } \\
\text { the logic circuitry for annunciation of } \\
\text { alert and alarm conditions as detected by } \\
\text { the CADMs. Located in CAP-Z9. }\end{array}$ & Per Drawing H-2-91179 & $\begin{array}{l}H-2-28883 \text { sht } 1 \\
H-2-28883 \text { sht } 2 \\
H-2-80143 \text { sht } 2\end{array}$ \\
\hline CALM-29-3 & $\begin{array}{l}\text { Criticality Alarm Logic Module. Provides } \\
\text { the logic circuitry for annunciation of } \\
\text { alert and alarm conditions as detected by } \\
\text { the CADMs. Located in CAP-29. }\end{array}$ & Per Drawing $H-2-91179$ & $\begin{array}{l}H-2-28883 \text { sht } 1 \\
H-2-28883 \text { sht } 2 \\
H-2-80143 \text { sht } 2\end{array}$ \\
\hline CALM-29-4 & $\begin{array}{l}\text { Criticality Alarm Logic Module. Provides } \\
\text { the logic circuitry for annunciation of } \\
\text { alert and alarm conditions as detected by } \\
\text { the CADMs. Located in CAP-Z9. }\end{array}$ & Per Drawing $\mathrm{H}-2-91179$ & $\begin{array}{l}H-2-28883 \text { sht } 1 \\
H-2-28883 \text { sht } 2 \\
H-2-80143 \text { sht } 2\end{array}$ \\
\hline CALM-29-5 & $\begin{array}{l}\text { Criticality Alarm Logic Module. Provides } \\
\text { the logic circuitry for annunciation of } \\
\text { alert and alarm conditions as detected by } \\
\text { the CADMs. Located in CAP-29. }\end{array}$ & Per Drawing $\mathrm{H}-2-91179$ & $\begin{array}{l}H-2-28883 \text { sht } 1 \\
H-2-28883 \text { sht } 2 \\
H-2-80143 \text { sht } 2\end{array}$ \\
\hline
\end{tabular}




\begin{tabular}{|c|c|c|c|}
\hline $\begin{array}{r}A P P \perp C A B 1 E \\
312 \\
32\end{array}$ & \multicolumn{3}{|c|}{ 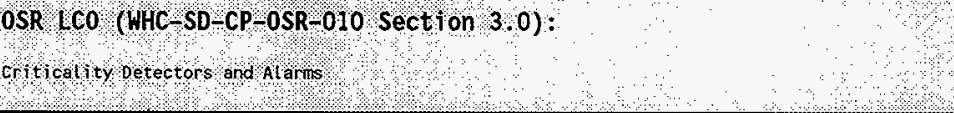 } \\
\hline 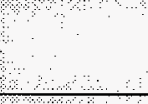 & $\begin{array}{r}\text { OSR SYSTEMEQUIPHENT } \\
\text { ZONE } 9\end{array}$ & OHPONENT LST & \\
\hline $\begin{array}{l}\text { MASTER } \\
\text { COMPONENT } \\
\text { TNDEX } \\
\text { NUMBER }\end{array}$ & 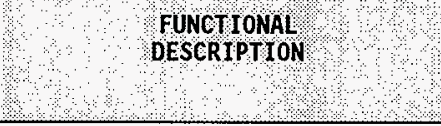 & $\begin{array}{l}\text { MANUFACTURER } \\
\text { PART/HODEL } \\
\text { NUMBER } \\
\text { (DWG }, 2,8014480145)\end{array}$ & $\begin{array}{l}\text { ESSENTIA } \\
\text { DRAHING } \\
\text { NUMBER }\end{array}$ \\
\hline CAP- 29 & $\begin{array}{l}\text { Criticality Alarm Panel Zone } 9 \text {. Contains } \\
\text { the CADMs, CALMs, CARM, Inhibit switches, } \\
\text { local (buitding) annunciation and Intertie } \\
\text { relays for the Zone } 9 \text { Criticality Alarm } \\
\text { System. Located in Building } 2736-28 \\
\text { Room } 638 \text {. }\end{array}$ & $\begin{array}{l}\text { Premier TVA } 7719-23 \text {, Relay } \\
\text { Rack } \\
\text { One Bay, Standard } 19 " \mathrm{~W} \times \\
23 " \mathrm{D} \\
2.125 " \text { Trim }\end{array}$ & $\begin{array}{l}H-2-28883 \text { sht } 1 \\
H-2-28883 \text { sht } 2 \\
H-2-80143 \text { sht } 2\end{array}$ \\
\hline CARM- 29 & $\begin{array}{l}\text { Criticality Alarm Relay Module. Activates } \\
\text { the alarm signal generator and provides an } \\
\text { annunciation signal in the event of a high } \\
\text { alarm signal from the CALM. Includes an } \\
\text { inhibit switch to disable the alarm output } \\
\text { signal. Located in CAP-Z9. }\end{array}$ & Per Drawing $\mathrm{H}-2-91180$ & $\begin{array}{l}H-2-28883 \text { sht } 1 \\
H=2-28883 \text { sht } 2 \\
H=2-80143 \text { sht } 2\end{array}$ \\
\hline CBS-Z9-1 & $\begin{array}{l}\text { Centrat Battery system. Nominal } 24 \text { VDC } \\
\text { system. Includes one } 12 \text { cell battery } \\
\text { capable of supplying power to the } \\
\text { criticality alarm system in excess of } 8 \\
\text { hours and charger capable of maintaining } \\
\text { the charge on the battery and while } \\
\text { providing normal power. Primary supply } \\
\text { power failure annunciation. Located } \\
\text { adjacent to cap-29. }\end{array}$ & $\begin{array}{l}\text { Battery: Exide } 3 \mathrm{CC}-5 \text {, } \\
\text { 24VDC } 100 \mathrm{~A} \\
\text { Charger: Exide UC } 26-25 \text {, } \\
24 \text { VDC, } 25 \mathrm{~A}\end{array}$ & $\begin{array}{l}H-2-28883 \text { sht } 1 \\
H-2-28883 \text { sht } 2 \\
H-2-80143 \text { sht } 2\end{array}$ \\
\hline $\begin{array}{c}\text { AC POWER } \\
\text { RELAY } \\
\text { (Not required } \\
\text { for labeling } \\
\text { at this } \\
\text { time.) }\end{array}$ & $\begin{array}{l}\text { (SC-3 SE). AC Power Monitor Relay. } \\
\text { Deenergizes on loss of power. A manual } \\
\text { reset is required to prevent a fluctuating } \\
\text { (erratic) power supply from initiating } \\
\text { false alarms. }\end{array}$ & Allen Bradley \# 700-C200-A1 & $\begin{array}{l}H-2-28883 \text { sht } 1 \\
H-2-28883 \text { sht } 2 \\
H-2-80143 \text { sht } 2\end{array}$ \\
\hline$S-9-1 A$ & $\begin{array}{l}\text { Inhibit switch for CAD/CAOM-Z9-1A used to } \\
\text { prevent annunciation of alarm during test, } \\
\text { calibration, and replacement of CADS and } \\
\text { CADMS. Located on CAP-Z9. }\end{array}$ & $\begin{array}{l}\text { Square } D \text { Class } 9001 \text {, } \\
\text { Type LRSA-2K1 with } \\
\text { Type LA-1 Contact Blocks }\end{array}$ & $\begin{array}{l}H-2-28883 \text { sht } 1 \\
H-2-28883 \text { sht } 2 \\
H-2-80143 \text { sht } 2\end{array}$ \\
\hline$s-9-18$ & $\begin{array}{l}\text { Inhibit Switch for CAD/CADM-29-1B used to } \\
\text { prevent annunciation of alarm during test, } \\
\text { calibration, and replacement of CADS and } \\
\text { CADMs. Located on CAP-Z9. }\end{array}$ & $\begin{array}{l}\text { Square D Class } 9001, \\
\text { Type LRSA-2K1 with } \\
\text { Type LA-1 Contact Blocks }\end{array}$ & $\begin{array}{l}H-2-28883 \text { sht } 1 \\
H-2-28883 \text { sht } 2 \\
H-2-80143 \text { sht } 2\end{array}$ \\
\hline$s-9-1 C$ & $\begin{array}{l}\text { Inhibit switch for CAD/CADM-29-1C used to } \\
\text { prevent annunciat ion of alarm during test, } \\
\text { calibration, and replacement of CADS and } \\
\text { CADMs. Located on CAP-Z9. }\end{array}$ & $\begin{array}{l}\text { Square D Class } 9001 \text {, } \\
\text { Type LRSA-2K1 with } \\
\text { Type LA-1 Contact Blocks }\end{array}$ & $\begin{array}{l}H-2-28883 \text { sht } 1 \\
H-2-28883 \text { sht } 2 \\
H-2-80143 \text { sht } 2\end{array}$ \\
\hline
\end{tabular}




\begin{tabular}{|c|c|c|c|}
\hline \multicolumn{4}{|c|}{ 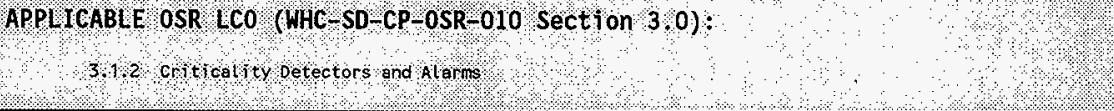 } \\
\hline$\therefore$ & 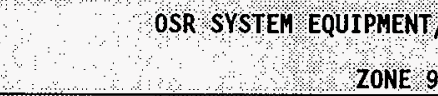 & ONPONENT LIST & \\
\hline $\begin{array}{l}\text { WASTER: } \\
\text { COMPONENT } \\
\text { NONDEX } \\
\text { NUMBER }\end{array}$ & POUNCT IONAW PCRTPTION & $\begin{array}{l}\text { WANUFACTURER } \\
\text { PARTHODEL } \\
\text { NUNBER } \\
\text { (DWG }+2-80144 / 80145)\end{array}$ & $\begin{array}{l}\text { ESSENTYA } \\
\text { DRAWING } \\
\text { NUMBER }\end{array}$ \\
\hline$S-9-2 A$ & $\begin{array}{l}\text { Inhibit Switch for CAD/CADM- } 29-2 A \text { used to } \\
\text { prevent annunciation of alarm during test, } \\
\text { calibration, and replacement of CADs and } \\
\text { CADMs. Located on CAP-Z9. }\end{array}$ & $\begin{array}{l}\text { Square D Class } 9001 \text {, } \\
\text { Type LRSA-2K1 with } \\
\text { Type LA-1 Contact Blocks }\end{array}$ & $\begin{array}{l}H-2-28883 \text { sht } 1 \\
H-2-28883 \text { sht } 2 \\
H-2-80143 \operatorname{sht} 2\end{array}$ \\
\hline$s-9-2 B$ & $\begin{array}{l}\text { Inhibit Switch for CAD/CADM- } 29-2 B \text { used to } \\
\text { prevent annunciation of alarm during test, } \\
\text { calibration, and replacement of CADs and } \\
\text { CADMs. Located on CAP- } 29 \text {. }\end{array}$ & $\begin{array}{l}\text { Square D Class } 9001, \\
\text { Type LRSA-2K1 with } \\
\text { Type LA-1 Contact Blocks }\end{array}$ & $\begin{array}{l}H-2-28883 \text { sht } 1 \\
H-2-28883 \text { sht } 2 \\
H-2-80143 \text { sht } 2\end{array}$ \\
\hline$s-9-2 c$ & $\begin{array}{l}\text { Inhibit Switch for CAD/CADM- } 29-2 C \text { used to } \\
\text { prevent annunciation of alarm during test, } \\
\text { calibration, and replacement of CADs and } \\
\text { CADMs. Located on CAP-Z9. }\end{array}$ & $\begin{array}{l}\text { Square D Class } 9001, \\
\text { Type LRSA-2K1 with } \\
\text { Type LA-1 Contact Blocks }\end{array}$ & $\begin{array}{l}H-2-28883 \text { sht } 1 \\
H-2-28883 \text { sht } 2 \\
H-2-80143 \text { sht } 2\end{array}$ \\
\hline$s-9-3 A$ & $\begin{array}{l}\text { Inhibit Switch for CAD/CADM-Z9-3A used to } \\
\text { prevent annunciation of alarm during test, } \\
\text { cal ibration, and replacement of CADs and } \\
\text { CADMs. Located on CAP-Z9. }\end{array}$ & $\begin{array}{l}\text { Square D Class } 9001 \text {, } \\
\text { Type LRSA-2K1 with } \\
\text { Type LA-1 Contact Blocks }\end{array}$ & $\begin{array}{l}H-2-28883 \text { sht } 1 \\
H-2-28883 \text { sht } 2 \\
H-2-80143 \text { sht } 2\end{array}$ \\
\hline$s-9-3 B$ & $\begin{array}{l}\text { Inhibit Switch for CAD/CADM-Z9-3B used to } \\
\text { prevent annunciation of alarm during test, } \\
\text { cal ibration, and replacement of CADs and } \\
\text { CADMs. Located on CAP-Z9. }\end{array}$ & $\begin{array}{l}\text { Square D Class } 9001, \\
\text { Type LRSA-2K1 with } \\
\text { Type LA-1 Contact Blocks }\end{array}$ & $\begin{array}{l}H-2-28883 \text { sht } 1 \\
H-2-28883 \text { sht } 2 \\
H-2-80143 \text { sht } 2\end{array}$ \\
\hline$s-9-3 c$ & $\begin{array}{l}\text { Inhibit Switch for CAD/CADM-Z9-3C used to } \\
\text { prevent annunciation of alarm during test, } \\
\text { calibration, and replacenent of CADs and } \\
\text { CADHs. Located on CAP-Z9. }\end{array}$ & $\begin{array}{l}\text { Square D Class } 9001, \\
\text { Type LRSA-2K1 with } \\
\text { Type LA-1 Contact Blocks }\end{array}$ & $\begin{array}{l}H-2-28883 \text { sht } 1 \\
H-2-28883 \text { sht } 2 \\
H-2-80143 \text { sht } 2\end{array}$ \\
\hline$S-9-4 A$ & $\begin{array}{l}\text { Inhibit Switch for CAD/CADM-Z9-4A used to } \\
\text { prevent annunciation of alarm during test, } \\
\text { cal ibration, and replacement of CADs and } \\
\text { CADMs. Located on CAP-Z9. }\end{array}$ & $\begin{array}{l}\text { Square } D \text { Class } 9001, \\
\text { Type LRSA-2K1 with } \\
\text { Type LA-1 Contact Blocks }\end{array}$ & $\begin{array}{l}H-2-28883 \text { sht } 1 \\
H-2-28883 \text { sht } 2 \\
H-2-80143 \text { sht } 2\end{array}$ \\
\hline$s-9-4 B$ & $\begin{array}{l}\text { Inhibit switch for CAD/CADM- } 29-4 \mathrm{~B} \text { used to } \\
\text { prevent annunciation of alarm during test, } \\
\text { cal ibration, and replacement of CADS and } \\
\text { CADMs. Located on CAP-29. }\end{array}$ & $\begin{array}{l}\text { Square } D \text { Class } 9001, \\
\text { Type LRSA-2K1 with } \\
\text { Type LA-1 Contact Blocks }\end{array}$ & $\begin{array}{l}H-2-28883 \text { sht } 1 \\
H-2-28883 \text { sht } 2 \\
H-2-80143 \text { sht } 2\end{array}$ \\
\hline
\end{tabular}




\begin{tabular}{|c|c|c|c|}
\hline \multirow{2}{*}{\multicolumn{4}{|c|}{ 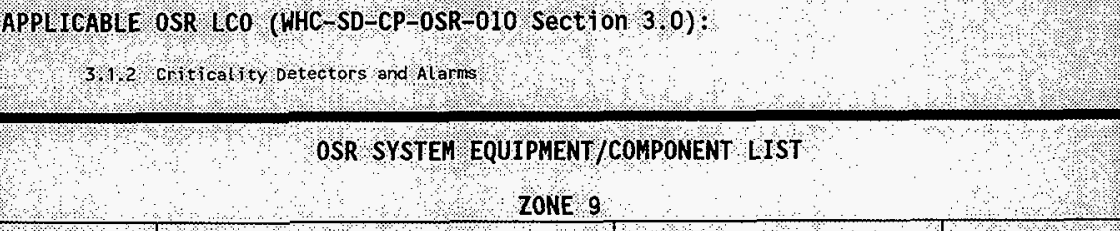 }} \\
\hline & & & \\
\hline $\begin{array}{l}\text { MASTER } \\
\text { COMPONENT } \\
\text { TINOEX } \\
\text { NUMBER }\end{array}$ & FUNCT IONAL & $\begin{array}{l}\text { MANUFACTURER } \\
\text { PART/HODEL } \\
\text { NUMBER } \\
\text { (DWg } 12-80144 / 80145)\end{array}$ & $\begin{array}{l}\text { ESSENT TAL } \\
\text { DRAWTIG } \\
\text { NGMBER }\end{array}$ \\
\hline$s-9-4 c$ & $\begin{array}{l}\text { Inhibit Switch for CAD/CADM-Z9-4C used to } \\
\text { prevent annunciation of alarm during test, } \\
\text { cal ibration, and replacement of CADS and } \\
\text { CADMs. Located on CAP- } 29 \text {. }\end{array}$ & $\begin{array}{l}\text { Square D Class } 9001 \text {, } \\
\text { Type LRSA-2K1 with } \\
\text { Type LA-1 Contact Blocks }\end{array}$ & $\begin{array}{l}H-2-28883 \text { sht } 1 . \\
H-2-28883 \text { sht } 2 \\
H-2-80143 \text { sht } 2\end{array}$ \\
\hline$s-9-5 A$ & $\begin{array}{l}\text { Inhibit Switch for CAD/CAOM-Z9-5A used to } \\
\text { prevent annunciation of alarm during test, } \\
\text { cal ibration, and replacement of CADS and } \\
\text { CADMs. Located on CAP-Z9. }\end{array}$ & $\begin{array}{l}\text { Square D Class } 9001 \text {, } \\
\text { Type LRSA-2K1 with } \\
\text { Type LA-1 Contact Blocks }\end{array}$ & $\begin{array}{l}H-2-28883 \text { sht } 1 \\
H-2-28883 \text { sht } 2 \\
H-2-80143 \text { sht } 2\end{array}$ \\
\hline$s-9-5 B$ & $\begin{array}{l}\text { Inhibit Switch for CAD/CADM-Z9-5B used to } \\
\text { prevent annunciation of alarm during test, } \\
\text { calibration, and replacement of CADS and } \\
\text { CADMs. Located on CAP-Z9. }\end{array}$ & $\begin{array}{l}\text { Square D Class } 9001 \text {, } \\
\text { Type LRSA-2K1 with } \\
\text { Type LA-1 Contact Blocks }\end{array}$ & $\begin{array}{l}H-2-28883 \text { sht } 1 \\
H-2-28883 \text { sht } 2 \\
H-2-80143 \text { sht } 2\end{array}$ \\
\hline$s-9-5 c$ & $\begin{array}{l}\text { Inhibit Switch for CAD/CADM-Z9-5C used to } \\
\text { prevent annunciation of alarm during test, } \\
\text { cal ibration, and replacement of CADS and } \\
\text { CADMs. Located on CAP-Z9. }\end{array}$ & $\begin{array}{l}\text { Square D Class } 9001 \text {, } \\
\text { Type LRSA-2K1 with } \\
\text { Type LA-1 Contact Blocks }\end{array}$ & $\begin{array}{l}H-2-28883 \text { sht } 1 \\
H-2-28883 \text { sht } 2 \\
H-2-80143 \text { sht } 2\end{array}$ \\
\hline
\end{tabular}




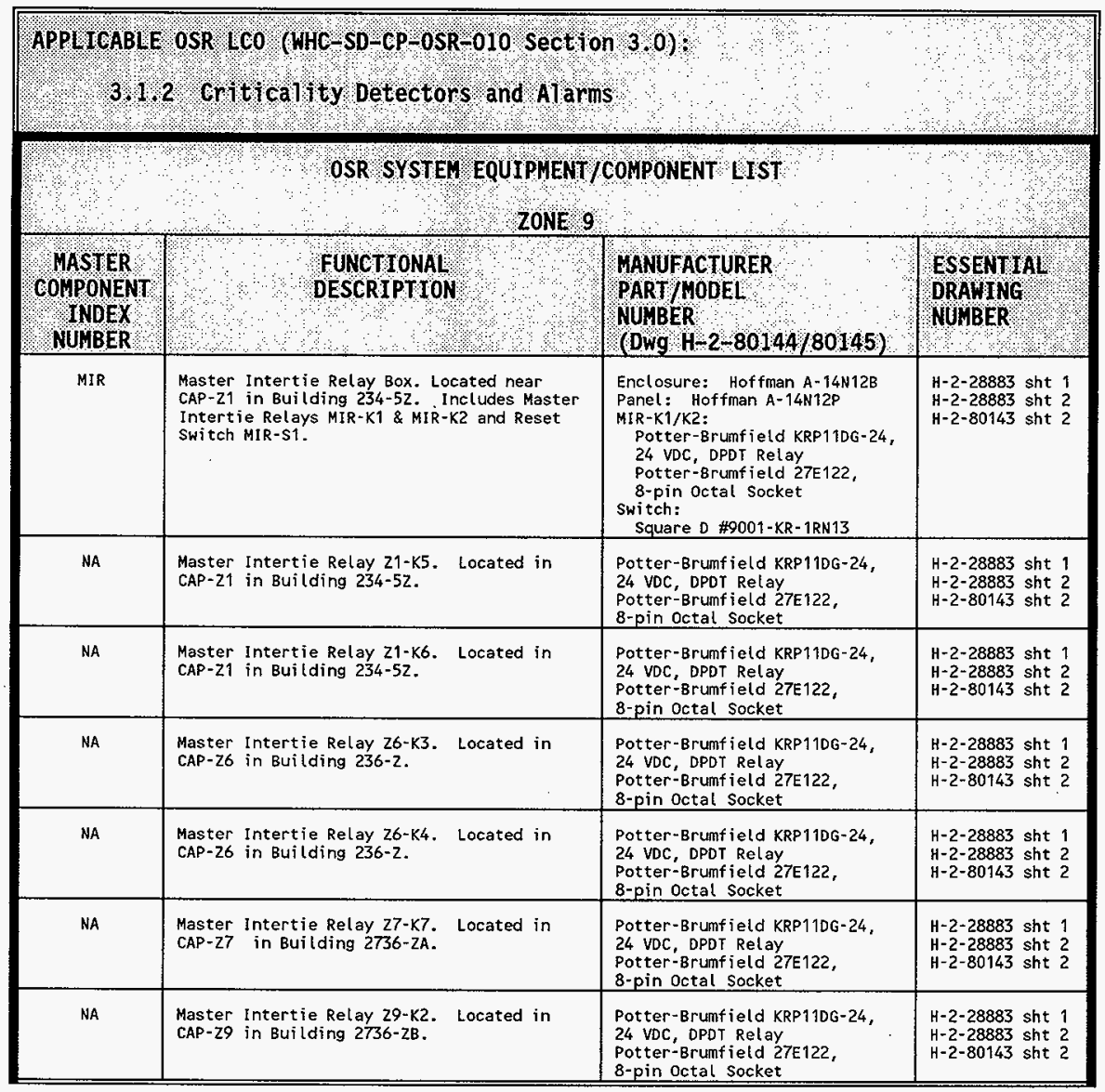




\section{APPENDIX C}

CAS SAFETY ENVELOPE BOUNDARY DRAWINGS

The following drawing defines the Safety Envelope Components for the Criticality Alarm System.

H-2-28883 Sheet 1, Criticality Alarm System Block Diagram and Drawing Index H-2-28505 (classified CNSI) 
APPENDIX D

CAS ESSENTIAL DRAWINGS

The following drawings are ESSENTIAL for the Criticality Alarm System.

H-2-28083 sheet 9, NUCLEAR CRITICALITY ALARM SYSTEM [B1dg 2736-Z, 2736-ZA]

H-2-28883 sheet 2, CRITICALITY ALARM SYSTEM BLOCK DIAGRAM AND DRAWING INDEX

H-2-28885 sheet 7, CRITICALITY ALARM SYSTEM FOUNDATION \& TUNNEL [B7dg 234-5Z]

H-2-28885 sheet 8, CRITICALITY ALARM SYSTEM FIRST FL00R [B1dg 234-5Z]

$\mathrm{H}-2-28885$ sheet 9, CRITICALITY ALARM SYSTEM DUCT LEVEL [B1dg 234-5Z]

$\mathrm{H}-2-28885$ sheet 10 , CRITICALITY ALARM SYSTEM SECOND FLOOR [B1dg 234-5Z]

$\mathrm{H}-2-28885$ sheet 11, CRITICALITY ALARM SYSTEM ROOF [B1dg 234-5Z]

H-2-28888 sheet 2, CRITICALITY ALARM SYSTEM FLOOR PLAN [B]dg 236-Z]

$\mathrm{H}-2-80143$ sheet 2, CRITICALITY ALARM SYSTEM [B1dg 2721-Z, 2736-ZB]

$\mathrm{H}-2-91823$ sheet 6 , CRITICALITY ALARM SYSTEM FLOOR PLAN [B1dg 241-Z] 


\section{DISTRIBUTION SHEET}

\begin{tabular}{|c|c|c|c|c|c|}
\hline \multirow{2}{*}{$\begin{array}{l}\text { To } \\
\text { PLUTONIUM FINISHING PLANT } \\
\text { (PFP) FACILITY }\end{array}$} & \multirow{2}{*}{\multicolumn{3}{|c|}{$\begin{array}{l}\text { From } \\
\text { PFP ENGINEERING }\end{array}$}} & \multicolumn{2}{|l|}{ Page 1 of 1} \\
\hline & & & & \multicolumn{2}{|c|}{ Date $5 / 12 / 97$} \\
\hline \multicolumn{6}{|l|}{ Project Title/Work Order } \\
\hline \multicolumn{4}{|c|}{$\begin{array}{l}\text { DEFINITION AND MEANS OF MAINTAINING THE CRITICALITY DETECTORS } \\
\text { AND ALARMS PORTION OF THE PLUTONIUM FINISHING PLANT SAFETY } \\
\text { ENVELOPE }\end{array}$} & \multicolumn{2}{|c|}{ ECN No. 634415} \\
\hline Name & MSIN & $\begin{array}{l}\text { Text } \\
\text { With All } \\
\text { Attach. }\end{array}$ & Text Only & $\begin{array}{l}\text { Attach.f } \\
\text { Appendix } \\
\text { Only }\end{array}$ & $\begin{array}{c}\text { EDT/ECN } \\
\text { Only }\end{array}$ \\
\hline
\end{tabular}

G. G. BERGQUIST

B. M. BUTTS

D. A. CONNERS

L. E. EDVALSON

M. W. GIBSON

G. A. GLOVER

D. R. GROTH

J. M. HELD

D. D. JOHNSON

R. D. KECK

J. P. KING

c. M. KRONVALL

E. M. LAROCK

D. J. McBRIDE

S. E. NUNN

D.-M.PPANKAt

A. L. RAMBLE

R. D. REDEKOPP

H. E. REW

J. R. ROBERTSON

T5-55 X

T5-54

T5-11

T5-48

T5-55

T4-20

T4-15

T5-11

T4-20

T4-20

T5-51

T5- 15

T5-02

T5-15

T5-11

$-74-36$

T5-54

T5-15

T4-15

T5-54

C. A. SALINAS

T5-02

R. W. SZEMPRUCH

T5-48

D. W. TEMPLETON

R3-79

W. F. WHITE

G. P. WILSON

D. M. WYATT

CENTRAL FILES

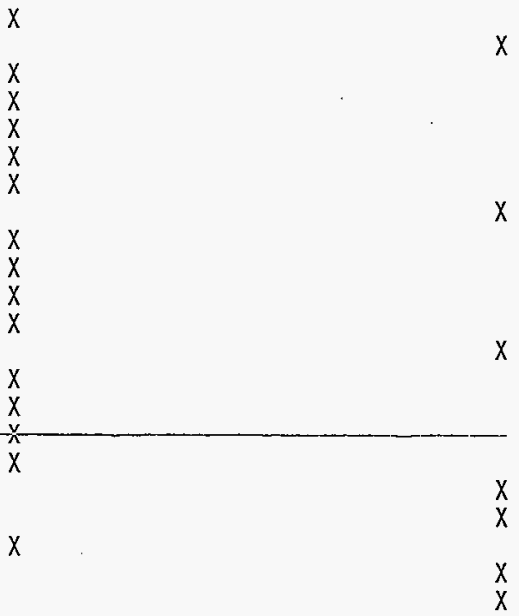

T4-20

T4-20

T5-11

$x$

2 Copies

L8-04 